UNIVERSIDADE DE SÃO PAULO ESCOLA DE ENGENHARIA DE SÃO CARLOS

Departamento de Engenharia de Estruturas

\title{
Técnicas de amostragem inteligente em simulação de Monte Carlo
}



KETSON ROBERTO MAXIMIANO DOS SANTOS

\title{
Técnicas de amostragem inteligente em simulação de Monte Carlo
}

\author{
VERSÃO CORRIGIDA \\ A versão original encontra-se na Escola de Engenharia de São Carlos
}

Dissertação apresentada à Escola de Engenharia de São Carlos da Universidade de São Paulo, como parte dos requisitos para a obtenção do Título de Mestre em Ciências, Programa de Engenharia Civil (Engenharia de Estruturas).

Orientador: Prof. Assoc. André Teófilo Beck 


\section{AUTORIZO A REPRODUÇÃO TOTAL OU PARCIAL DESTE TRABALHO, POR QUALQUER MEIO CONVENCIONAL OU ELETRÔNICO, PARA FINS DE ESTUDO E PESQUISA, DESDE QUE CITADA A FONTE.}

Monte Carlo / Ketson Roberto Maximiano dos Santos; orientador André Teófilo Beck. São Carlos, 2014.

Dissertação (Mestrado) - Programa de Pós-Graduação em Engenharia de Estruturas -- Escola de Engenharia de São Carlos da Universidade de São Paulo, 2014.

1. Confiabilidade de Estruturas. 2. Método de Monte Carlo. 3. Amostragem Inteligente. I. Título. 


\section{FOLHA DE JULGAMENTO}

Candidato: Engenheiro KETSON ROBERTO MAXIMIANO DOS SANTOS.

Título da dissertação: "Técnicas de amostragem inteligente em simulação de Monte Carlo".

Data da defesa: $26 / 03 / 2014$

\section{Comissão Julgadora:}

Prof. Associado André Teófilo Beck(Orientador)

(Escola de Engenharia de São Carlos/EESC)

Prof. Dr. Marcelo Areias Trindade

(Escola de Engenharia de São Carlos/EESC)

Prof. Dr. Rafael Holdorf Lopez

(Universidade Federal de Santa Catarina/UFSC)
Resultado:
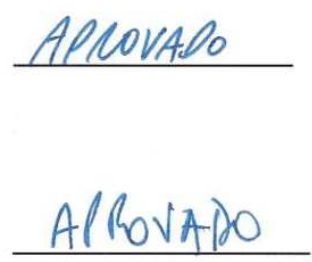

APROVADO

Coordenadora do Programa de Pós-Graduação em Engenharia Civil (Engenharia de Estruturas):

Profa. Associada Ana Lucia Homce de Cresce El Debs

Presidente da Comissão de Pós-Graduação:

Prof. Titular Denis Vinicius Coury 

À minha mãe Cícera, à meu pai José Roberto e em especial à minha avó Nair Rosa (in memoriam) 



\section{AGRADECIMENTOS}

Agradeço primeiramente a Deus pela sabedoria dada, necessária para a conclusão de mais esta etapa. Aos meus pais Cícera e José Roberto, por todo o apoio e dedicação durante esta caminhada. À minha tia Luzia, por ser tão presente e preocupada. A toda minha família, pelo apoio incondicional, mesmo nos momentos difíceis. À minha namorada Hélia, que apesar de minha ausência e distância se mostrou companheira pelo incentivo e dedicação para que esse trabalho pudesse ser concluído com êxito.

Ao professor André Beck, pelos ensinamentos, incentivo e disponibilidade, fundamentais no desenvolvimento deste trabalho. Aos professores Rodrigo Ribeiro Paccola e Edson Denner Leonel, pelos bons comentários durante o exame de qualificação e pela disponibilidade. Ao professor Wellison Gomes, pela disponibilidade em sanar algumas dúvidas e pelas sugestões dadas. À banca examinadora formada pelos professores Rafael Holdorf Lopez e Marcelo Areias Trindade pelas sugestões dadas durante a defesa.

Aos Amigos do Departamento de Engenharia de Estruturas da Escola de Engenharia de São Carlos, pelos bons momentos vividos na cidade de São Carlos. Aos amigos da "República Alagoas", Cleilson Bernardino, Emerson Acácio, Gregório Felipe, Marcell Gustavo, Nichollas e Ricardo Sampaio por esse tempo de partilha e convivência. Aos amigos André Vieira, Carlinhos Moreira, Arthur Álax, Pablo Augusto, Rafael Niño, Sérgio Callejas, Hugo Oliveira, Matheus Fernandes, Fernando Vecchio, Elias Testoni, Laurenn Borges e Henrique Kroetz. À banda cigana que de maneira geral alegrou os churrascos nas repúblicas São Carlenses.

Aos professores e amigos do departamento de Física da Universidade Federal de Alagoas, Tássius Maciel, Eliel Gomes, José Maria, Túlio Amâncio, Eduardo Macena, professor Carlos Jacinto e professora Maria Tereza. Aos amigos e professores do Centro de Tecnologia (CTEC) da Universidade Federal de Alagoas, do Laboratório de Computação Científica e Visualização (LCCV) e do Programa de Formação de Recursos Humanos da ANP (PRH-40), em especial ao Heleno Pontes, à Ynaê Almeida e ao Lucas Gouveia.

E a tantos outros que não cabem em apenas uma folha.

Ao CNPq pelo auxilio financeiro. 



\section{RESUMO}

SANTOS, K. R. M. Técnicas de amostragem inteligente em simulação de Monte Carlo. Dissertação (Mestrado - Engenharia de Estruturas), 2014 - Escola de Engenharia de São Carlos, Universidade de São Paulo, São Paulo, 2014.

A confiabilidade de estruturas apresenta sólidos desenvolvimentos teóricos e crescentes aplicações práticas. Durante os últimos anos, avanços significativos foram obtidos em termos dos métodos de transformação (FORM, SORM), bem como em termos das técnicas de simulação de Monte Carlo. Métodos de transformação se mostraram eficientes para problemas de dimensões e não-linearidades moderadas. Já técnicas de simulação sempre permitiram a solução de problemas de grandes dimensões e fortemente não lineares, embora o custo computacional possa ser uma séria limitação. Com o avanço da capacidade de processamento dos computadores e com o desenvolvimento de técnicas de amostragem inteligente, a simulação de Monte Carlo passa a ser cada vez mais viável. Este trabalho tem por objetivo estudar e programar em computador técnicas de amostragem inteligente em simulação de Monte Carlo. O StRAnD é um programa de computador que já possui implementadas as técnicas de simulação de Monte Carlo Bruto e com Amostragem por Importância, ambas utilizando a Amostragem Simples na geração das variáveis básicas. Assim, são adicionadas, ao StRAnD, as técnicas de Amostragem Assintótica, Amostragem Melhorada e Simulação de Subconjuntos. Além disso, são programadas as técnicas de Amostragem por Hipercubo Latino e Amostragem por Variáveis Antitéticas. Nesta dissertação, são analisados seis problemas distintos, de forma que as vantagens e desvantagens de cada técnica sejam avaliadas, em termos da probabilidade de falha, do coeficiente de variação da probabilidade de falha, do erro relativo da probabilidade de falha e do tempo de processamento.

Palavras-chave: Confiabilidade de Estruturas, Método de Monte Carlo, Amostragem Inteligente. 



\begin{abstract}
SANTOS, K. R. M. Intelligent sampling techniques in Monte Carlo simulation. Dissertation (Master - Structural Engineering), 2014 - São Carlos School of Engineering, University of São Paulo, São Paulo, 2014.

The structural reliability presents solid theoretical developments and increasing practical applications. During the past few years, significant advances were achieved in terms of transformation methods (FORM and SORM), as well as, in terms of Monte Carlo Simulation. Transformation methods are effective in problems with moderate dimensions and moderate nonlinearities. On the other hand, simulation techniques can be used to solve highdimensional problems and highly nonlinear problems, although the computational cost could be a serious limitation. With the progress of computer processing capacity and with the development of intelligent sampling techniques, the Monte Carlo Simulation becomes increasingly feasible. This work aims to study and program intelligent sampling techniques in Monte Carlo simulation. The StRAnD (Structural Reliability Analysis and Design) software already has Crude Monte Carlo and Importance Sampling Monte Carlo, both using Simple Sampling as basic samples generator. Thus, the Asymptotic Sampling technique, the Enhanced Sampling technique and the Subset Simulation were added to the software. Moreover, the Latin Hypercube Sampling technique and the Antithetic Variates techniques were also added to the software. Six problems were evaluated in order to evaluate the advantages and disadvantages of each technique, in terms of probability of failure, coefficient of variation of the probability of failure, relative error and processing time.
\end{abstract}

Keywords: Structural Reliability, Monte Carlo method, Intelligent Sampling. 



\section{LISTA DE FIGURAS}

Figura 1. Equação de estado limite e domínios de falha e segurança (BECK, 2012)................. 52

Figura 2. Teste de uniformidade: a) $i=1.000$ e b) $i=10.000$ (à esquerda).................................... 63

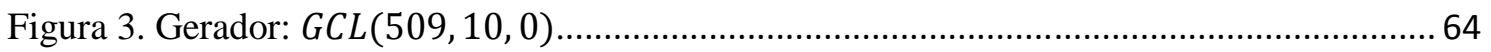

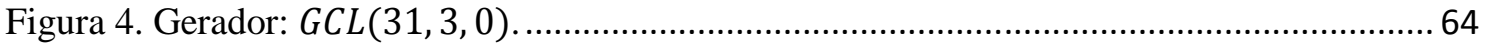

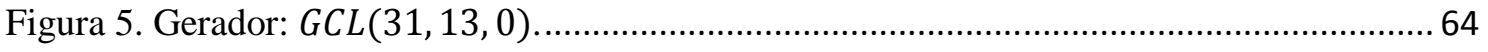

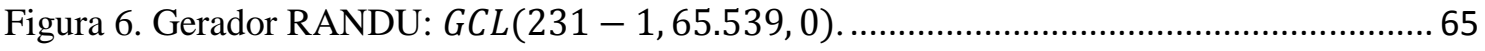

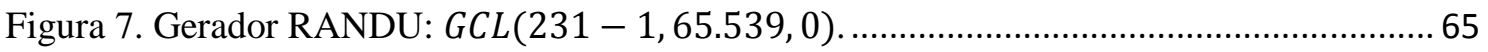

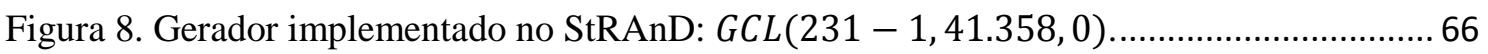

Figura 9. Geração de números aleatórios com distribuição prescrita (BECK, 2012). ................. 67

Figura 10. Gráfico de convergência da probabilidade de falha pela simulação do Monte Carlo Bruto,

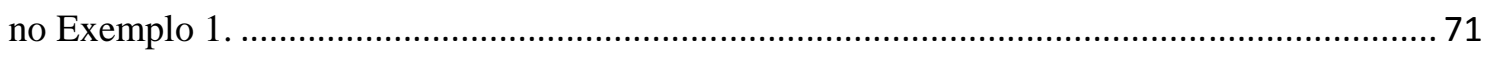

Figura 11. Amostragem estratificada (HURTADO; BARBAT, 1998)..................................... 73

Figura 12. Amostragem por hipercubo latino (HURTADO; BARBAT, 1998). ......................... 73

Figura 13. Hipercubo Latino para duas variáveis e cinco realizações (OLSSON; SANDEBERG;

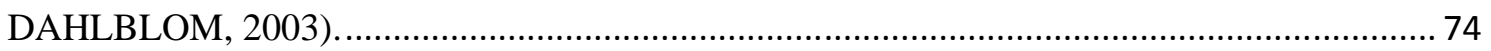

Figura 14. Histograma de frequência na a) Amostragem Simples, b) Amostragem por Variáveis Antitéticas e c) Amostragem por Hipercubo Latino. .................................................................. 75

Figura 15. Comparativo da convergência do Monte Carlo Bruto com a Amostragem por Importância, no Exemplo 1.

Figura 16. Convergência do Monte Carlo com Amostragem por Importância, no Exemplo 1... 77

Figura 17. Vetor do ponto de projeto. 78

Figura 18. Hipercubo Latino original e transformado, adaptado de (OLSSON; SANDEBERG e

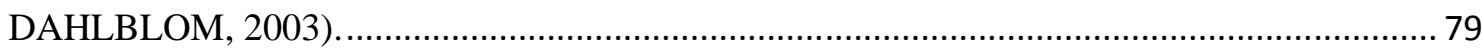

Figura 19. Amostragem por importância utilizando pontos de projeto (BECK, 2012)................ 80

Figura 20. Ilustração do ajuste não linear realizado nas duplas $f, \beta f$.

Figura 21. Parametrização dos desvios-padrão das variáveis aleatórias (SICHANI; NIELSEN;

BUCHER, 2011b).

Figura 22. Distribuições de probabilidade parametrizadas das variáveis: Capacidade (C) e Demanda (D). 
Figura 23. Geração de subconjuntos via cadeias de Markov.......

Figura 24. Regressão não linear utilizada na Amostragem Assintótica para amostras de tamanho $2 \times 10^{3}$, no Exemplo 1 . 95

Figura 25. Regressão não linear utilizada na Amostragem Assintótica para amostras de tamanho $10^{5}$, no Exemplo 1. .95

Figura 26. Regressão não linear utilizada na Amostragem Melhorada para amostras de tamanho $2 \times 10^{3}$, no Exemplo 1. 97

Figura 27. Regressão não linear utilizada na Amostragem Melhorada para amostras de tamanho $10^{5}$, no Exemplo 1. 97

Figura 28. Subconjuntos gerados na Simulação de Subconjuntos, para o Exemplo 1. 100

Figura 29. Comparativo da convergência da $P_{f}$ (a) e de C.V. (b) para a Amostragem Simples, no Exemplo 1 101

Figura 30. Comparativo da convergência da $P_{f}$ (a) e de C.V. (b) para a Amostragem por Hipercubo Latino, no Exemplo 1. 101

Figura 31. Comparativo da convergência da $P_{f}$ (a) e de C.V. (b) para a Amostragem por Variáveis Antitéticas, no Exemplo 1. 101

Figura 32. Comparativo da convergência da $P_{f}$ (a) e de C.V. (b) para o Monte Carlo Bruto, no Exemplo 1 102

Figura 33. Comparativo da convergência da $P_{f}($ a) e de C.V. (b) para a Amostragem por Importância, no Exemplo 1. 102

Figura 34. Comparativo da convergência da $P_{f}$ (a) e de C.V. (b) para a Amostragem Assintótica, no Exemplo 1 103

Figura 35. Comparativo da convergência da $P_{f}$ (a) e de C.V. (b) para a Amostragem Melhorada, no Exemplo 1 103

Figura 36. Comparativo da probabilidade de falha para uma amostra de tamanho 9.200, no Exemplo 1. 105

Figura 37. Relação entre o tempo de processamento e o tamanho da amostra no Exemplo 1: Monte Carlo Bruto (a) e Amostragem por Importância (b). 106

Figura 38. Relação entre o tempo de processamento e o tamanho da amostra no Exemplo 1: Amostragem Assintótica (a) e Amostragem Melhorada (b). 106

Figura 39. Relação entre o tempo de processamento e o tamanho da amostra no Exemplo 1: Simulação de Subconjuntos (a) e comparativo entre todas as técnicas(b). 106

Figura 40. FDP da variável Demanda (D) e FDA da variável Capacidade (C), para o Exemplo 2.108

Figura 41. Curva a ser integrada no cálculo da probabilidade de falha, no Exemplo 2. 109 
Figura 42. Regressão não linear na Amostragem Assintótica, para o Exemplo 2.

Figura 43. Espaço amostral parametrizado utilizando a Amostragem Assintótica, no Exemplo 2.110

Figura 44. Relação entre o índice de confiabilidade, relativo à area do domínio de falha, e o parâmetro $f$, na Amostragem Assintótica, para Exemplo 2.

Figura 45. Regressão não linear na Amostragem Melhorada, para o Exemplo 2.

Figura 46. Limite de falha parametrizado, para o Exemplo 2.

Figura 47. Área relativa do domínio de falha parametrizado, para o Exemplo 2.

Figura 48. Subconjuntos gerados via cadeias de Markov por meio do algoritmo de Metropolis-

Hastings Modificado. 114

Figura 49. Comparativo da convergência de $P_{f}$ para o Monte Carlo Bruto com Amostragem Simples e com Amostragem por Hipercubo Latino, para o Exemplo 2. 114

Figura 50. Comparativo da convergência de $P_{f}$ para o Monte Carlo Bruto com Amostragem Simples e com Amostragem por Variáveis Antitéticas, para o Exemplo 2. 115

Figura 51. Comparativo da convergência de $P_{f}$ para o Monte Carlo Bruto com Amostragem por Hipercubo Latino e com Amostragem por Variáveis Antitéticas, para o Exemplo 2. 115

Figura 52. Coeficiente de variação da probabilidade de falha na Amostragem por Importância, para o Exemplo 2. 116

Figura 53. Comparativo da probabilidade de falha para uma amostra de tamanho 3.680, no Exemplo 2. 117

Figura 54. Relação entre o tempo de processamento e o tamanho da amostra no Exemplo 2: comparativo entre todas as técnicas (a) e Monte Carlo Bruto (b). 118

Figura 55. Limite de falha e os domínios de falha e segurança, para o Exemplo 3. 118

Figura 56. Regressão não linear utilizada na Amostragem Assintótica para amostras de tamanho $3 \times 10^{3}$, no Exemplo 3 119

Figura 57. Regressão não linear utilizada na Amostragem Assintótica para amostras de tamanho $10^{5}$, no Exemplo 3. 120

Figura 58. Regressão não linear utilizada na Amostragem Melhorada para amostras de tamanho $3 \times 10^{3}$, no Exemplo 3. 121

Figura 59. Regressão não linear utilizada na Amostragem Melhorada para amostras de tamanho $10^{5}$, no Exemplo 3. 121

Figura 60. Simulação de Subconjuntos: Amostragem Simples, no Exemplo 3. 123

Figura 61. Simulação de Subconjuntos: Amostragem por Hipercubo Latino, no Exemplo 3. . 123

Figura 62. Simulação de Subconjuntos: Amostragem por Variáveis Antitéticas, no Exemplo 3.123 
Figura 63. Comparativo da convergência da $P_{f}$ (a) e de C.V. (b) para a Amostragem Simples, no Exemplo 3.

Figura 64. Comparativo da convergência da $P_{f}$ (a) e de C.V. (b) para a Amostragem por Hipercubo Latino, no Exemplo 3. 124

Figura 65. Comparativo da convergência da $P_{f}$ (a) e de C.V. (b) para a Amostragem por Variáveis Antitéticas, no Exemplo 3.

Figura 66. Comparativo da convergência da $P_{f}$ (a) e de C.V. (b) para o Monte Carlo Bruto, no Exemplo 3. 125

Figura 67. Comparativo da convergência da $P_{f}($ a) e de C.V. (b) para a Amostragem por Importância, no Exemplo 3.

Figura 68. Comparativo da convergência da $P_{f}($ a) e de C.V. (b) para a Amostragem Assintótica, no Exemplo 3 126

Figura 69. Comparativo da convergência da $P_{f}$ (a) e de C.V. (b) para a Amostragem Melhorada, no Exemplo 3. 126

Figura 70. Comparativo da probabilidade de falha para uma amostra de tamanho 5.550, no Exemplo 3.

Figura 71. Relação entre o tempo de processamento e o tamanho da amostra no Exemplo 3: Monte Carlo Bruto (a) e Amostragem por Importância (b).

Figura 72. Relação entre o tempo de processamento e o tamanho da amostra no Exemplo 3:

Amostragem Assintótica (a) e Amostragem Melhorada (b).... .130

Figura 73. Relação entre o tempo de processamento e o tamanho da amostra no Exemplo 3: Simulação de Subconjuntos (a) e comparativo entre todas as técnicas(b). 130

Figura 74. Regressão não linear utilizada na Amostragem Assintótica para amostras de tamanho $10^{4}$, no Exemplo 4

Figura 75. Regressão não linear utilizada na Amostragem Assintótica para amostras de tamanho $10^{5}$, no Exemplo 4

Figura 76. Regressão não linear utilizada na Amostragem Melhorada para amostras de tamanho $10^{4}$, no Exemplo 4 133

Figura 77. Regressão não linear utilizada na Amostragem Melhorada para amostras de tamanho $10^{5}$, no Exemplo 4 134

Figura 78. Comparativo da convergência da $P_{f}$ (a) e de C.V. (b) para a Amostragem Simples, no Exemplo 4 135

Figura 79. Comparativo da convergência da $P_{f}$ (a) e de C.V. (b) para a Amostragem por Hipercubo Latino, no Exemplo 4 
Figura 80. Comparativo da convergência da $P_{f}$ (a) e de C.V. (b) para a Amostragem por Variáveis Antitéticas, no Exemplo 4.

Figura 81. Comparativo da convergência da $P_{f}$ (a) e de C.V. (b) para o Monte Carlo Bruto, no Exemplo 4.

Figura 82. Comparativo da convergência da $P_{f}$ (a) e de C.V. (b) para a Amostragem por Importância, no Exemplo 4.

Figura 83. Comparativo da convergência da $P_{f}$ (a) e de C.V. (b) para a Amostragem Assintótica, no Exemplo 4. 138

Figura 84. Comparativo da convergência da $P_{f}($ a) e de C.V. (b) para a Amostragem Melhorada, no Exemplo 4. 138

Figura 85. Comparativo da probabilidade de falha para uma amostra de tamanho 2.300, no Exemplo 4. 140

Figura 86. Relação entre o tempo de processamento e o tamanho da amostra no Exemplo 4: Monte Carlo Bruto (a) e Amostragem por Importância (b).

Figura 87. Relação entre o tempo de processamento e o tamanho da amostra no Exemplo 4: Amostragem Assintótica (a) e Amostragem Melhorada (b).

Figura 88. Relação entre o tempo de processamento e o tamanho da amostra no Exemplo 4: Simulação de Subconjuntos (a) e comparativo entre todas as técnicas(b). 141

Figura 89.Ilustração da treliça hiperestática estudada..... 143

Figura 90. Convergência para a Amostragem por Importância, no Exemplo 5. 145

Figura 91. Regressão não linear utilizada na Amostragem Assintótica para amostras de tamanho $10^{3}$, no Exemplo 5. 145

Figura 92. Regressão não linear utilizada na Amostragem Assintótica para amostras de tamanho $10^{5}$, no Exemplo 5. 146

Figura 93. Regressão não linear utilizada na Amostragem Melhorada para amostras de tamanho $10^{3}$, no Exemplo 5. 147

Figura 94. Regressão não linear utilizada na Amostragem Melhorada para amostras de tamanho $10^{5}$, no Exemplo 5. 147

Figura 95. Comparativo da convergência da $P_{f}$ (a) e de C.V. (b) para a Amostragem Simples, no Exemplo 5. 149

Figura 96. Comparativo da convergência da $P_{f}$ (a) e de C.V. (b) para a Amostragem por Hipercubo Latino, no Exemplo 5. 149

Figura 97. Comparativo da convergência da $P_{f}$ (a) e de C.V. (b) para a Amostragem por Variáveis Antitéticas, no Exemplo 5. 
Figura 98. Comparativo da convergência da $P_{f}$ (a) e de C.V. (b) para o Monte Carlo Bruto, no Exemplo 5.

Figura 99. Comparativo da convergência da $P_{f}($ a) e de C.V. (b) para a Amostragem Assintótica, no Exemplo 5. 151

Figura 100. Comparativo da convergência da $P_{f}($ a) e de C.V. (b) para a Amostragem Melhorada, no Exemplo 5 151

Figura 101. Comparativo da probabilidade de falha para uma amostra de tamanho 3.700, no Exemplo 5 .

Figura 102. Relação entre o tempo de processamento e o tamanho da amostra no Exemplo 5: Monte Carlo Bruto (a) e Amostragem por Importância (b). 153

Figura 103. Relação entre o tempo de processamento e o tamanho da amostra no Exemplo 5: Amostragem Assintótica (a) e Amostragem Melhorada (b). 154

Figura 104. Relação entre o tempo de processamento e o tamanho da amostra no Exemplo 5:

Simulação de Subconjuntos (a) e comparativo entre todas as técnicas(b). 154

Figura 105. Torre analisada e nós de referência, para o Exemplo 6. 155

Figura 106. Diagrama fator de carga $\times$ deslocamento. 156

Figura 107. Regressão não linear utilizada na Amostragem Assintótica, para amostras de tamanho $10^{5}$, no Exemplo 6. 159

Figura 108. Regressão não linear utilizada na Amostragem Melhorada para amostras de tamanho $10^{5}$, no Exemplo 6 160

Figura 109. Simulação de Subconjuntos: Amostragem Simples. 162

Figura 110. Simulação de Subconjuntos: Amostragem por Hipercubo Latino. .162

Figura 111. Simulação de Subconjuntos: Amostragem por Variáveis Antitéticas. 163

Figura 112. Comparativo da convergência da $P_{f}($ a) e de C.V. (b) para a Amostragem Simples, no Exemplo 6. 163

Figura 113. Comparativo da convergência da $P_{f}($ a) e de C.V. (b) para a Amostragem por Hipercubo Latino, no Exemplo 6. .164

Figura 114. Comparativo da convergência da $P_{f}$ (a) e de C.V. (b) para a Amostragem por Variáveis Antitéticas, no Exemplo 6. 164

Figura 115. Comparativo da convergência da $P_{f}$ (a) e de C.V. (b) para o Monte Carlo Bruto, no Exemplo 6 165

Figura 116. Comparativo da convergência da $P_{f}($ a) e de C.V. (b) para a Amostragem por Importância, no Exemplo 6 165 
Figura 117. Comparativo da convergência da $P_{f}$ (a) e de C.V. (b) para a Amostragem Assintótica, no Exemplo 6. 166

Figura 118. Comparativo da convergência da $P_{f}($ a) e de C.V. (b) para a Amostragem Melhorada, no Exemplo 6. 166

Figura 119. Comparativo da probabilidade de falha para uma amostra de tamanho 14.800, no Exemplo 6. 168

Figura 120. Comparativo dos tempos de processamento (em segundos) das tecnicas empregadas no Exemplo 6, para uma amostra de tamanho 14.800 170

Figura 121. Diagrama básico do StRAnD. 179

Figura 122. Dado de saída do StRAnD: Amostra aleatória a) 2-D e b) 3-D. 187

Figura 123. Dado de saída do StRAnD: geração de subconjuntos na simulação de subconjuntos, no Exemplo 1. 188

Figura 124. Dado de saída do StRAnD: Graficos de convergência, no Exemplo 1. 189

Figura 125. Dado de saída do StRAnD: Graficos de regressão utilizando o modelo do Exemplo 1. 190

Figura 126. Dado de saída do StRAnD: Ranking dos pontos amostrais do Hipercubo Latino Gerado. 



\section{LISTA DE TABELAS}

Tabela 1. Médias e desvios padrão das variáveis aleatórias do Exemplo 1.

Tabela 2. Parâmetros ajustados na Amostragem Assintótica, para o Exemplo 1. 96

Tabela 3. Probabilidade de falha e coeficiente de Variação da probabilidade de falha, para a Amostragem Assintótica, no Exemplo 1. 96

Tabela 4. Parâmetros ajustados na Amostragem Melhorada, para uma amostra de tamanho $2 \times 10^{3}$, no Exemplo 1. 98

Tabela 5. Parâmetros ajustados na Amostragem Melhorada, para uma amostra de tamanho $10^{5}$, no Exemplo 1. 98

Tabela 6. Probabilidade de falha e coeficiente de Variação da probabilidade de falha, para a Amostragem Melhorada, no Exemplo 1 98

Tabela 7. Médias e desvios padrão do caminhante aleatório do Exemplo 1 99

Tabela 8. Comparativo da $P_{f}$ para uma amostra de tamanho 9.200, no Exemplo 1 104

Tabela 9. Parâmetros das variáveis aleatórias do Exemplo 2. 108

Tabela 10. Médias e desvios padrão do caminhante aleatório do Exemplo 2. 113

Tabela 11. Comparativo da $P_{f}$ para uma amostra de tamanho 3.680, no Exemplo 2............. 116

Tabela 12. Médias e desvios padrão das variáveis aleatórias do Exemplo 3. 119

Tabela 13. Parâmetros ajustados na Amostragem Assintótica, para o Exemplo 3 120

Tabela 14. Probabilidade de falha e coeficiente de Variação da probabilidade de falha, para a Amostragem Assintótica, no Exemplo 3. 120

Tabela 15. Parâmetros ajustados na Amostragem Melhorada, para uma amostra de tamanho $3 \times 10^{3}$, no Exemplo 3. 122

Tabela 16. Parâmetros ajustados na Amostragem Melhorada, para uma amostra de tamanho $10^{5}$, no Exemplo 3.

Tabela 17. Probabilidade de falha e coeficiente de Variação da probabilidade de falha, para a Amostragem Melhorada, no Exemplo 3

Tabela 18. Médias e desvios padrão do caminhante aleatório do Exemplo 3. 122

Tabela 19. Comparativo da $P_{f}$ para uma amostra de tamanho 5.500, no Exemplo 3. 128

Tabela 20. Médias e desvios padrão das variáveis aleatórias do Exemplo 4. 131

Tabela 21. Parâmetros ajustados na Amostragem Assintótica, para o Exemplo 4 133 
Tabela 22. Probabilidade de falha e coeficiente de Variação da probabilidade de falha, para a Amostragem Assintótica, no Exemplo 4.

Tabela 23. Parâmetros ajustados na Amostragem Melhorada, para uma amostra de tamanho $10^{4}$, no Exemplo 4 134

Tabela 24. Parâmetros ajustados na Amostragem Melhorada, para uma amostra de tamanho $10^{5}$, no Exemplo 4

Tabela 25. Probabilidade de falha e coeficiente de Variação da probabilidade de falha, para a Amostragem Melhorada, no Exemplo 4 135

Tabela 26. Comparativo da $P_{f}$ para uma amostra de tamanho 2.300, no Exemplo 4. .139

Tabela 27. Propriedades dos perfis de aço utilizados no Exemplo 5 (Verzenhassi, 2008). .142

Tabela 28. Médias e coeficientes de variação das variáveis aleatórias do Exemplo 5. 144

Tabela 29. Parâmetros ajustados na Amostragem Assintótica para o Exemplo 5. 146

Tabela 30. Probabilidade de falha e coeficiente de Variação da probabilidade de falha, para a Amostragem Assintótica, no Exemplo 5. 146

Tabela 31. Parâmetros ajustados na Amostragem Melhorada, para uma amostra de tamanho $10^{3}$, no Exemplo 5.

Tabela 32. Parâmetros ajustados na Amostragem Melhorada, para uma amostra de tamanho $10^{5}$, no Exemplo 5.

Tabela 33. Probabilidade de falha e coeficiente de Variação da probabilidade de falha, para a Amostragem Melhorada, no Exemplo 5 .

Tabela 34. Comparativo da $P_{f}$ para uma amostra de tamanho 3.700, no Exemplo 5. .152

Tabela 35. Médias e coeficientes de variação das variáveis aleatórias do Exemplo 6. 157

Tabela 36. Comparativo do uso de diferentes técnicas de amostragem básica no método de Monte Carlo Bruto, para uma amostra de $10^{5}$, no exemplo 6 . 158

Tabela 37. Comparativo do uso de diferentes técnicas de amostragem básica no método de Monte Carlo com Amostragem por Importância, para uma amostra de $10^{5}$, no exemplo 6. 158

Tabela 38. Parâmetros ajustados na Amostragem Assintótica para o Exemplo 6. .160

Tabela 39. Índice de confiabilidade, probabilidade de falha, coeficiente de variação da probabilidade de falha e tempo de processamento, para a Amostragem Assintótica, no Exemplo 6. 160

Tabela 40. Parâmetros ajustados na Amostragem Melhorada, para uma amostra de tamanho $10^{5}$, no Exemplo 6. 161

Tabela 41. Índice de confiabilidade, probabilidade de falha, coeficiente de variação da probabilidade de falha e tempo de processamento para a Amostragem Melhorada, no Exemplo 6. 161 
Tabela 42. Médias e desvios padrão do caminhante aleatório do Exemplo 6

Tabela 43. Comparativo da $P_{f}$ para uma amostra de tamanho 14.800, no Exemplo 6. 167

Tabela 44. Comparativo da $P_{f}$, no Exemplo 6. 168

Tabela 45. Tempo de processamento para o Exemplo 6, para uma amostra de tamanho 14.800.169 



\section{LISTA DE SIGLAS}

AA - Amostragem Assintótica

AAM - Amostragem Melhorada com Amostragem por Variáveis Antitéticas

AASMC - Amostragem Assintótica com Amostragem por Variáveis Antitéticas

AI - Amostragem por Importância

AISMC - Amostragem por Importância com Amostragem por Variáveis Antitéticas

ALHS - Amostragem Assintótica com Amostragem por Hipercubo Latino

AM - Amostragem Melhorada/ Amostragem Melhorada com Amostragem Simples

AM_LHS - Amostragem Melhorada com Amostragem por Hipercubo Latino

ASIMC - Monte Carlo Bruto com Amostragem por Variáveis Antitéticas

ASMC - Amostragem Assintótica com Amostragem Simples

BRUTO - Monte Carlo Bruto

FDA - Função de Distribuição Acumulada

FDP - Função de Densidade de Probabilidade

FORM - First Order Reliability Method

GCL - Gerador congruente linear

ILHS - Amostragem por Importância com Amostragem por Hipercubo Latino

ISMC - Amostragem por Importância com Amostragem Simples

LHS - Latin Hypercube Sampling

MCMC - Markov Chain Monte Carlo

SIMC - Monte Carlo Bruto com Amostragem Simples

SLHS - Monte Carlo Bruto com Amostragem por Hipercubo Latino

SORM - Second Order Reliability Method

SS - Simulação de Subconjuntos/ Simulação de Subconjuntos com Amostragem Simples

SSA - Simulação de Subconjuntos com Amostragem por Variáveis Antitéticas

SSLHS - Simulação de Subconjuntos com Amostragem por Hipercubo Latino

StRAnD - Structural Reliability Analysis and Design 



\section{SUMÁRIO}

1. INTRODUÇÃ

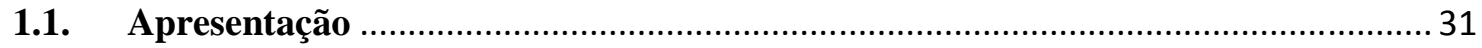

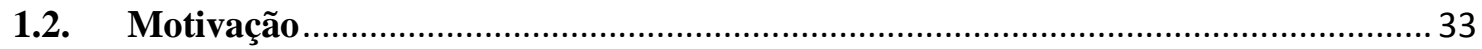

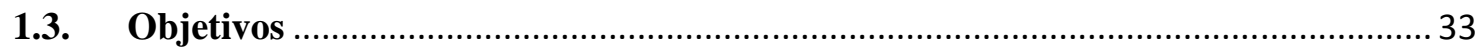

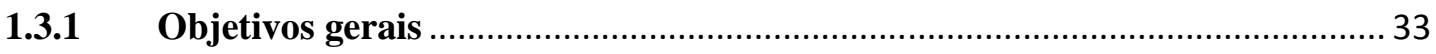

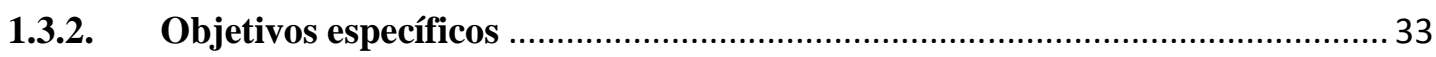

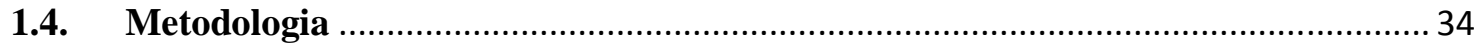

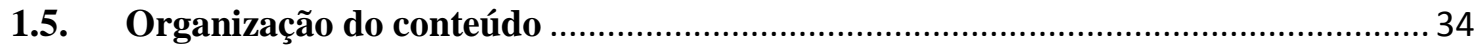

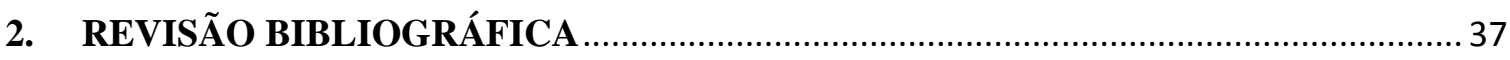

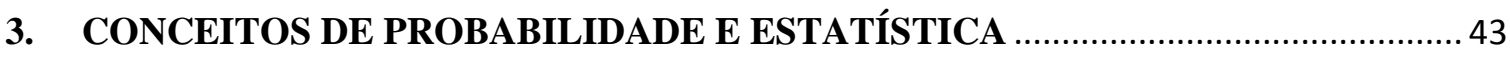

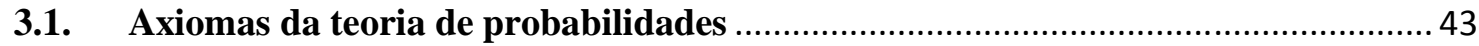

3.2. Probabilidades condicionais e independência de eventos ......................................... 44

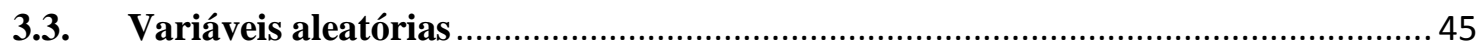

3.3.1. Função de distribuição acumulada de probabilidade ...................................... 45

3.3.2. Função densidade de probabilidade .............................................................. 46

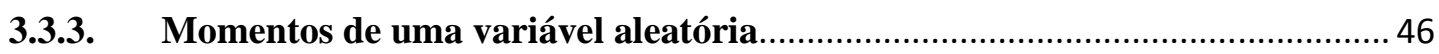

3.3.4. Média e variância de amostras.................................................................... 47

3.4. Distribuição conjunta de probabilidades ….......................................................... 48

3.4.1. Momentos conjuntos entre variáveis aleatórias............................................... 49

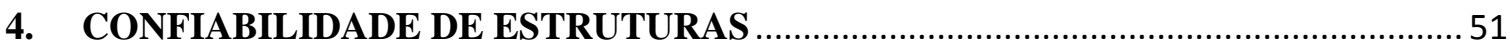

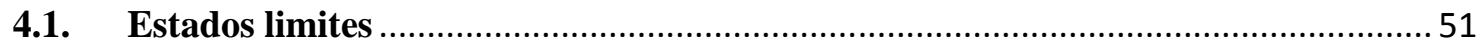

4.2. Problema fundamental da confiabilidade de estruturas..................................... 53

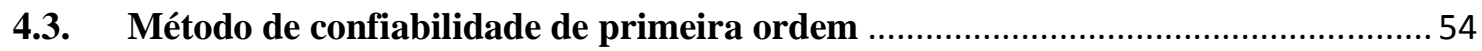

4.3.1. Transformação composta utilizando o modelo de Nataf ................................... 56

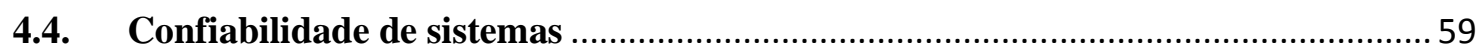

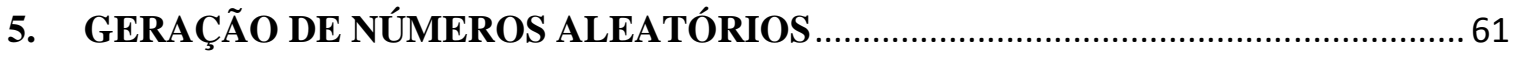

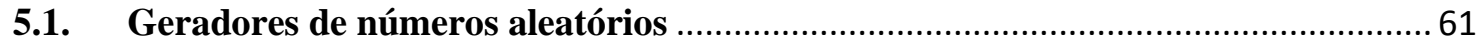

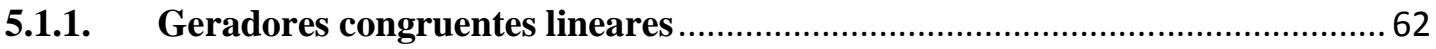

5.2. Geração de números aleatórios com distribuição de probabilidade prescrita ........66

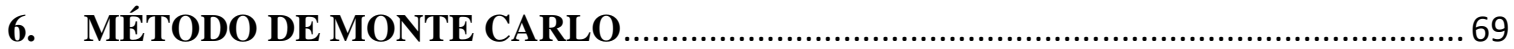

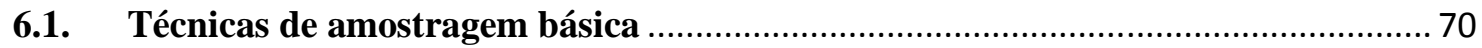




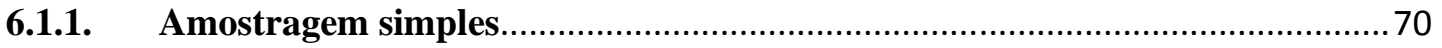

6.1.2. Amostragem por variáveis antitéticas …………………………………...... 72

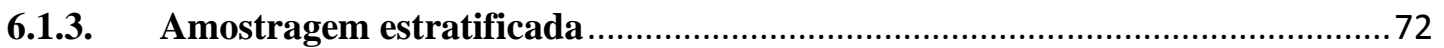

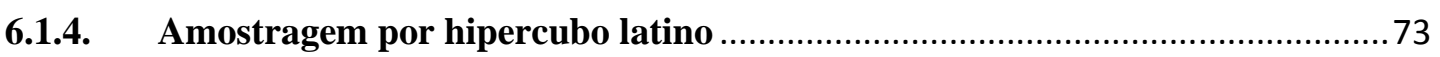

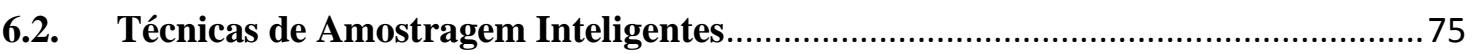

6.2.1. Amostragem por Importância utilizando pontos de projeto …………..............76

6.2.1.1. Amostragem por Importância utilizando Hipercubo Latino ..................................77

6.2.1.2. Múltiplos modos de falha ………………......................................................... 79

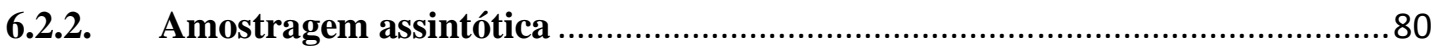

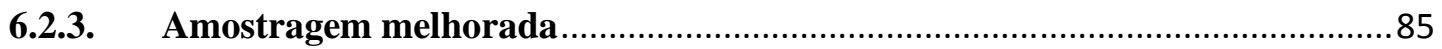

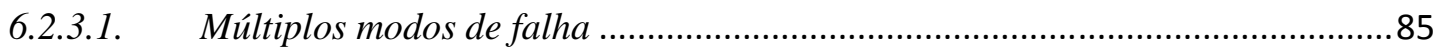

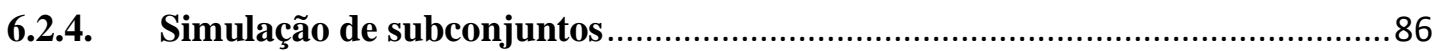

6.2.4.1. Coeficiente de Variação da probabilidade de falha ............................................89

6.2.4.2. Geração de Cadeias de Markov ........................................................................ 90

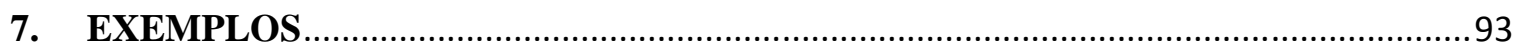

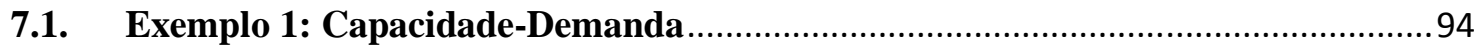

7.1.1. Amostragem Assintótica ……………................................................................ 95

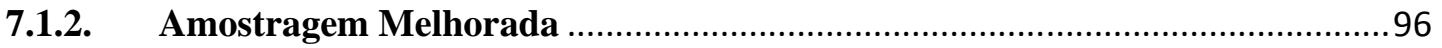

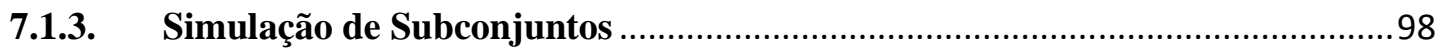

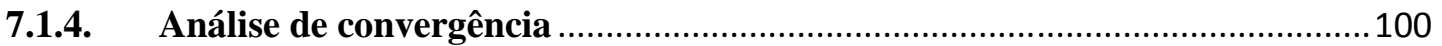

7.1.5. Comparativo da probabilidade de falha e de seu coeficiente de variação ......104

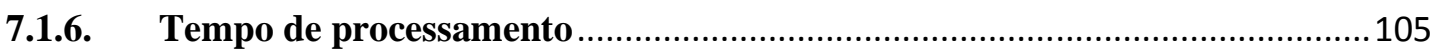

7.2. Exemplo 2: Variáveis Aleatórias Limitadas........................................................ 107

7.2.1. Amostragem Assintótica …………………................................................. 109

7.2.2. Amostragem Melhorada ……………….................................................... 111

7.2.3. Simulação de Subconjuntos ……….................................................................. 113

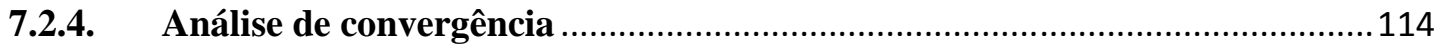

7.2.5. Comparativo da probabilidade de falha e de seu coeficiente de variação .....116

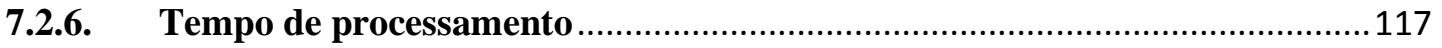

7.3. Exemplo 3: Equação de estado limite não linear ……………………......................

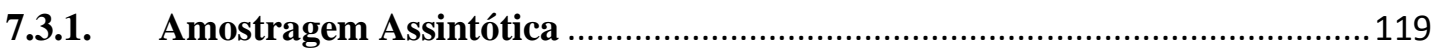

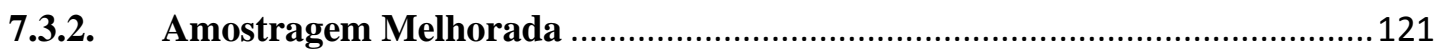

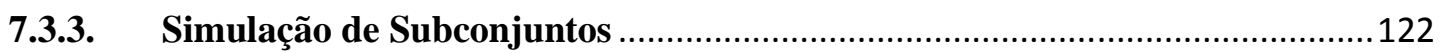

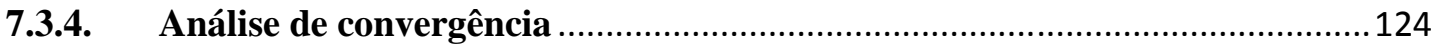


7.3.5. Comparativo da probabilidade de falha e de seu coeficiente de variação..... 127

7.3.6. Tempo de processamento $\quad 129$

7.4. Exemplo 4: Equação de estado limite não linear com variáveis aleatórias mistas130

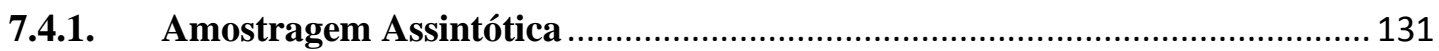

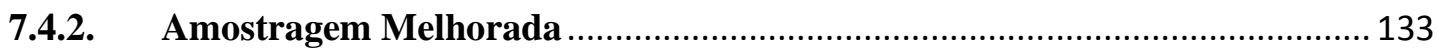

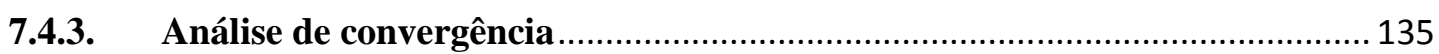

7.4.4. Comparativo da probabilidade de falha e de seu coeficiente de variação..... 139

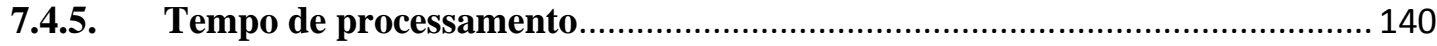

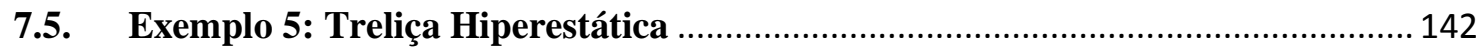

7.5.1. Amostragem por Importância....................................................................... 144

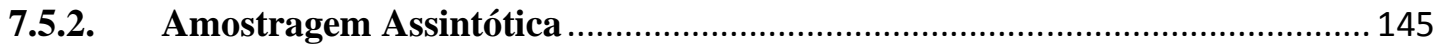

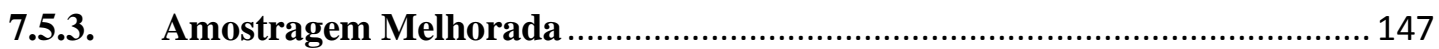

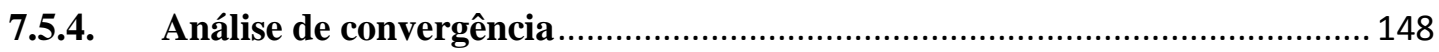

7.5.5. Comparativo da probabilidade de falha e de seu coeficiente de variação..... 151

7.5.6. Tempo de processamento

7.6. Exemplo 6: Torre em Elementos Finitos ........................................................... 154

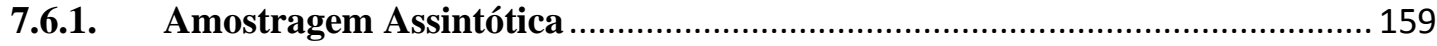

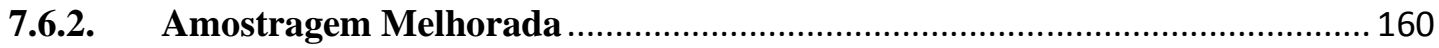

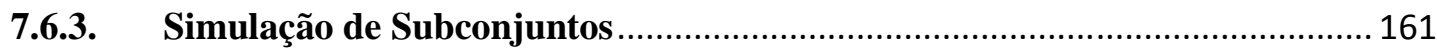

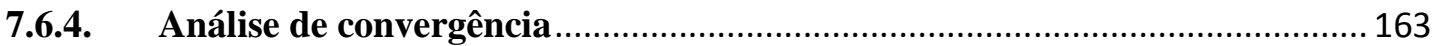

7.6.5. Comparativo da probabilidade de falha e de seu coeficiente de variação..... 167

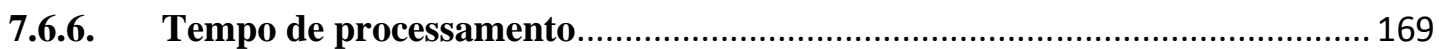

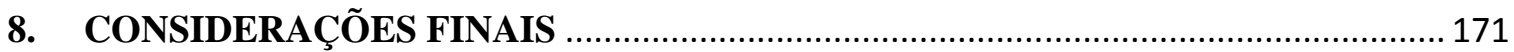

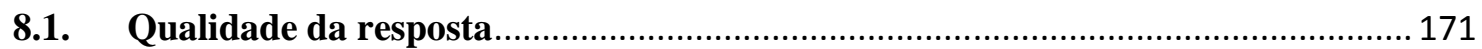

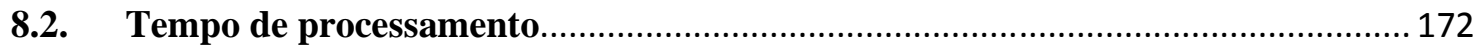

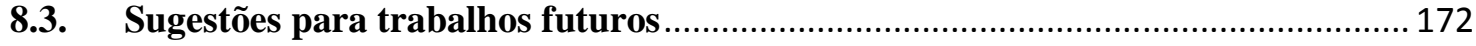

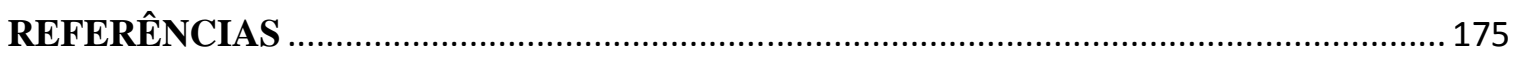

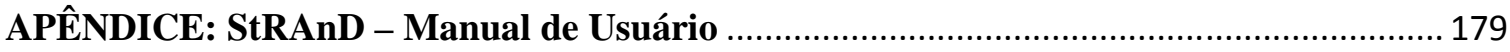

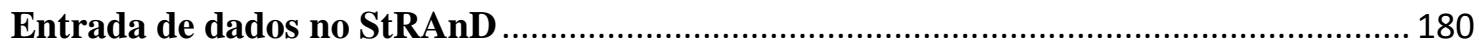

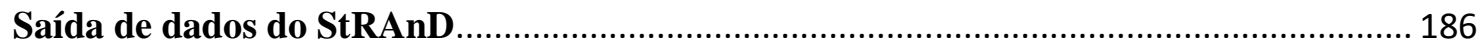





\section{INTRODUÇÃO}

\subsection{Apresentação}

Em engenharia, uma estrutura deve ser projetada de forma a obedecer alguns requisitos, tais como: serviço, segurança, robustez, econômico e social. Estes requisitos podem ser explicitados por meio de equações de estado limite, que determinam a fronteira entre o domínio de falha e de segurança de uma estrutura. Assim, ao considerarmos as incertezas que existem em cada parâmetro de projeto, uma análise determinística não é capaz de predizer o quão segura é a estrutura projetada. Desta forma, verifica-se a importância da aplicação de variabilidade às variáveis de projeto de engenharia, o que nos leva ao estudo da confiabilidade de estruturas (BECK, 2012).

A probabilidade de falha e o índice de confiabilidade são obtidos através da análise de confiabilidade de estruturas. Diversas técnicas têm sido desenvolvidas com o propósito de estimar tais valores. Dentre estas técnicas destacam-se o FORM (First Order Reliability Method) e o SORM (Second Order Reliability Method). Estas técnicas aproximam a equação de estado limite no ponto de projeto por um hiperplano, no caso do FORM, ou por uma hipersuperfície quadrática, no caso do SORM. A aproximação adotada pelo FORM e pelo SORM, para equação de estado limite, pode conduzir a erros, principalmente quando a equação de estado limite é fortemente não linear. Assim, pode-se fazer uso da simulação de Monte Carlo para se ter uma ideia do conteúdo de probabilidade que não está sendo levado em consideração na estimativa da probabilidade de falha.

Utilizando o FORM ou o SORM, pode-se encontrar o ponto de projeto, que vem a ser o ponto dentro do domínio de falha que possui o maior conteúdo de probabilidade, além disso, no espaço normal padrão ele é o ponto com a menor distância à origem deste espaço. Por isso, o ponto de projeto é bem indicado para se realizar a linearização da equação de estado limite no FORM; e para ser o ponto onde a função de amostragem é centrada, na técnica de Amostragem por Importância. Vale lembrar que na Amostragem por Importância, não é necessário que a função de amostragem seja centrada no ponto de projeto, porém, escolhas erradas deste ponto podem levar à obtenção de resultados não consistentes. Desta forma, quando não se pode definir bem o ponto de projeto, como nos casos em que a equação de 
estado limite apresenta pontos equidistantes da origem do espaço normal padrão, é necessário adotar técnicas de simulação. Todavia, técnicas de simulação podem ser proibitivas em problemas que apresentem probabilidade de falha pequena e que envolvam: sistemas estruturais, dimensão elevada, soluções numéricas não lineares, entre outros. Portanto, o estudo de técnicas de amostragem, que levem a obtenção de boas respostas em termos de probabilidade de falha com um menor esforço computacional, é de grande relevância dentro da confiabilidade de estruturas.

Um dos objetivos das técnicas de amostragem inteligente é estimar probabilidades de falha pequenas com a menor amostra possível. Entre as técnicas de amostragem inteligente podemos citar a Amostragem por Variáveis Antitéticas (Antithetic Variates) (HAMMERSLEY; MORTON, 1956), Amostragem por Importância (Importance Sampling), Amostragem por Hipercubo Latino (Latin Hypercube Sampling) (MCKAY; BECKMAN; CONOVER, 1979), a Amostragem Assintótica (Asymptotic Sampling) (BUCHER, 2009), Amostragem Melhorada (Enhanced Sampling) (NAESS; LEIRA; BATSEVYCH, 2009) e a Simulação de Subconjunto (Subset Simulation) (AU; BECK, 2001). Pode-se também utilizar algumas destas técnicas em conjunto.

A Amostragem por Variáveis Antitéticas busca inserir uma correlação negativa em relação a dois estimadores não tendenciosos da probabilidade de falha, reduzindo assim a variância da probabilidade de falha. A Amostragem por Importância utilizando pontos de projeto procura realizar uma amostragem na região mais propensa à falha, de forma que a estimativa da probabilidade de falha apresente uma menor variância. A Amostragem por Hipercubo Latino tem por objetivo gerar amostras esparsas, de forma que uma distribuição mais uniforme seja obtida. Assim, uma maior região do espaço amostral é coberta. A Amostragem Assintótica faz uso da propriedade assintótica que a probabilidade de falha tem à medida que os desvios padrão das variáveis aleatórias do problema tendem à zero. A técnica de Amostragem Melhorada procura parametrizar a função de estado limite e por meio de um procedimento de extrapolação a probabilidade de falha é estimada. Já a técnica de Simulação de Subconjuntos tem como ideia fundamental substituir uma probabilidade de falha pequena por um produto de probabilidades condicionais, desta forma, uma amostra menor é requerida, uma vez que as probabilidades condicionais são maiores que às do evento de falha original. 


\subsection{Motivação}

Em confiabilidade de estruturas não é raro nos depararmos com problemas em que as técnicas aproximativas falham na estimativa da probabilidade de falha. Assim, se recorre à simulação, como o método de Monte Carlo. Porém, nos problemas que possuem probabilidade de falha pequena, a utilização de amostragem simples pode levar a necessidade da geração de uma amostra aleatória muito grande. Tal fato pode ser proibitivo em alguns problemas, devido ao elevado custo computacional, tal como em problemas que envolvam a análise de modelos numéricos (e.g. Elementos Finitos, Elementos de Contorno, Diferenças Finitas e etc.).

No uso de modelos numéricos, o principal problema é que para cada simulação de Monte Carlo é necessário realizar uma simulação do modelo numérico. E se tais modelos numéricos utilizarem algoritmos iterativos (e.g. Newton-Raphson), que demandam um maior esforço computacional, o problema se acentua, pois, muitas vezes é necessário realizar várias simulações até que um evento raro seja observado.

Programar técnicas que viabilizem a solução dos problemas relatados anteriormente é uma tarefa de muita utilidade na confiabilidade de estruturas, e isto conduz aos objetivos do presente trabalho.

\subsection{Objetivos}

\subsubsection{Objetivos gerais}

Este trabalho tem por objetivo compilar, assimilar, programar em computador e comparar as técnicas de amostragem inteligente em simulação de Monte Carlo.

\subsubsection{Objetivos específicos}

1. Estudar, compreender e descrever as diferentes técnicas de amostragem inteligente em simulação de Monte Carlo;

2. Programar as técnicas estudadas, no módulo de simulação de Monte Carlo do programa computacional StRAnD - Structural Reliability Analysis and Design, que é escrito em linguagem Fortran com conceitos de programação orientada à objeto em sua versão estável e atual; 
3. Apresentar um estudo comparativo das técnicas estudadas, em problemas que são críticos no que se refere à estimativa da probabilidade de falha.

\subsection{Metodologia}

É realizada uma revisão bibliográfica com a finalidade de se encontrar e catalogar o que tem sido realizado nos últimos anos em relação às técnicas de amostragem inteligente em simulação de Monte Carlo. Além disso, é realizada uma busca dos estudos que deram origem a tais técnicas.

As técnicas de amostragem inteligente são programadas no módulo de simulação de Monte Carlo do StRAnD.

Neste trabalho são estudadas as seguintes técnicas de estimativa da probabilidade de falha:

- Monte Carlo Bruto;

- Monte Carlo com Amostragem por Importância utilizando pontos de projeto;

- Amostragem Assintótica;

- Amostragem Melhorada;

- Simulação de Subconjuntos.

As técnicas citadas podem ser empregadas com o uso da Amostragem Simples, da Amostragem por Hipercubo Latino e da Amostragem por Variáveis Antitéticas, o que propicia um bom número de combinações entre as técnicas.

Após a programação das técnicas, é realizado um estudo comparativo do desempenho destas, de maneira que as qualidades e deficiências sejam bem compreendidas. Isso servirá de base para que os usuários do StRAnD sejam capazes de utilizar as diferentes técnicas de amostragem inteligente na resolução de diversos problemas de confiabilidade de estruturas.

\subsection{Organização do conteúdo}

- Capítulo 2: É apresentada uma breve revisão bibliográfica sobre o tema em estudo, onde são citadas algumas obras fundamentais de forma a inserir o leitor no universo da simulação de Monte Carlo e da confiabilidade de estruturas.

- Capítulo 3: São abordados os conceitos referentes à teoria de probabilidades. 
- Capítulo 4: São apresentados os conceitos que envolvem confiabilidade de estruturas.

- Capítulo 5: É abordada a geração de números aleatórios.

- Capítulo 6: É apresentado o método de Monte Carlo e são apresentadas as técnicas de amostragem inteligente.

- Capítulo 7: São apresentados alguns problemas a serem resolvidos com as técnicas estudadas. São realizados estudos comparativos em forma de gráficos e tabelas.

- Capítulo 8: São apresentadas as considerações finais.

- Apêndice: É realizada uma descrição de como é feita a entrada e a saída de dados do StRAnD. 


\section{REVISÃO BIBLIOGRÁFICA}

Metropolis e Ulam (1949), no artigo intitulado de The Monte Carlo method, descrevem um método que é essencialmente uma abordagem estatística do estudo de equações integrais e diferenciais que ocorrem em vários ramos das ciências naturais. Os autores passam a distinguir os fenômenos que tratam da interação entre partículas, que é descrito pela mecânica clássica, daqueles que envolvem um número grande de partículas e que é descrito pela mecânica estatística. $O$ trabalho enfoca o estudo do conjunto de pontos que passa a ser descrito pela teoria de conjuntos. Também é relatado um exemplo em análise combinatória e teoria de probabilidades, que é o cálculo da probabilidade de êxito no jogo de paciência (solitaire). Tal problema é tomado de maneira meramente ilustrativa e trata da geração de diversos eventos e da avaliação da probabilidade de sucesso. Outro exemplo dado é a estimativa do volume de uma região no espaço $\mathbb{R}^{20}$, onde a região é definida pelo seguinte conjunto de inequações.

$$
f_{1}\left(x_{1}, x_{2}, \ldots, x_{20}\right)<0 ; f_{2}\left(x_{1}, x_{2}, \ldots, x_{20}\right)<0 ; \ldots ; f_{20}\left(x_{1}, x_{2}, \ldots, x_{20}\right)<0
$$

onde se considera que todos os pontos $x_{1}, x_{2}, \ldots, x_{20}$ satisfazem as inequações apresentadas anteriormente. Assim, Metropolis e Ulam (1949) afirmam que se a região é localizada em um cubo multidimensional unitário, a avaliação de integrais multidimensionais acaba se tornando uma tarefa difícil, por exemplo, dividindo-se cada coordenada $x_{i}$ em dez partes seria necessário avaliar uma grade com $10^{20}$ pontos no cubo unitário. Então, os autores relatam que seria melhor se fossem tomados aleatoriamente $10^{4}$ pontos e que aqueles que satisfizerem as inequações fossem contados. Outra ilustração apresentada pelos autores se refere à entrada de raios cósmicos na atmosfera, onde colisões entre partículas levam a gerações de outras partículas à medida que a probabilidade de geração destas partículas, com uma dada energia, depende apenas da energia da partícula que gerou a colisão anterior. Além disso, existe uma distribuição para a direção dos movimentos das partículas, que é caracterizado por cadeias de Markov.

Metropolis et al. (1953), no artigo intitulado de Equation of state calculation by fast computing machines, apresentam um método geral para investigar propriedades de substâncias consistindo de moléculas individuais interagentes. Eles consideram tal método 
como uma modificação da integração de Monte Carlo. O problema inicial consiste em estudar um método geral para um potencial arbitrário entre partículas. Assim, considerando um número finito de partículas $N$ em um quadrado, a energia potencial do sistema pode ser calculada como a soma das energias potenciais entre moléculas, que por sua vez é função da distância entre as partículas. Segundo os autores, para calcular o valor de equilíbrio do sistema, seriam necessárias integrações em um espaço $2 N$-dimensional, o que pode ser impraticável quando se tem um sistema com centenas de partículas. Assim, uma opção é utilizar o método de Monte Carlo na avaliação das integrais, de forma que colocando $N$ partículas no espaço $2 \mathrm{~N}$-dimensional, o movimento para os próximos estados dependem do estado anterior, tal estado é definido por uma caminhada aleatória centrada na posição original, de forma que qualquer ponto no espaço pode ser alcançado.

Hastings (1970), em seu artigo Monte Carlo sampling methods using Markov chains and their applications, apresenta uma generalização do método descrito por Metropolis et al. (1953) para superar as dificuldades que o método de Monte Carlo Bruto pode apresentar em problemas de grandes dimensões, que nesse caso seria o custo computacional na gerações de uma amostra aleatória grande. $\mathrm{O}$ autor apresenta a formulação básica do método e a geração de pontos amostrais via cadeias de Markov.

Robert e Casella (2011) apresentam uma revisão histórica sobre o método de Monte Carlo com Cadeias de Markov (Markov Chain Monte Carlo - MCMC) no artigo intitulado A short history of Markov Chain Monte Carlo: Subjective recollections from incomplete data. Os autores traçam a história do método de Monte Carlo com Cadeias de Markov desde os anos de 1940 até o período que o artigo foi publicado.

McKay, Beckman e Conover (1979), no artigo A comparison of three methods for selecting values of input variables in the analysis of output from a computer code, apresentam o desenvolvimento da Amostragem Estratificada e da Amostragem por Hipercubo Latino (Latin Hypercube Sampling-LHS). Na técnica de amostragem estratificada, todas as porções do espaço amostral de $\mathbf{X}$ são representadas pelos valores de entrada. A Amostragem por Hipercubo Latino, segundo os autores, garante que todas as porções do espaço amostral de $\mathbf{X}$ sejam representadas, de forma que o intervalo de cada variável aleatória é dividido em estratos com probabilidade $1 / N$ e cada um destes estratos é amostrado uma vez.

Olsson, Sandberg e Dahlblom (2003), no artigo intitulado de On latin hypercube sampling for structural reliability analysis, apresentam um comparativo entre diferentes 
técnicas de Amostragem por Importância, com ou sem uso do Hipercubo Latino. Foram analisadas seis técnicas, que são o método de Monte Carlo com Amostragem por Importância, Amostragem por Importância com Hipercubo Latino, Amostragem por Importância com Hipercubo Latino Transformado, Amostragem por Importância Axial, Amostragem por Importância Axial com Hipercubo Latino e Amostragem por Importância Axial com Hipercubo Latino com Correlação Reduzida. São estudados três exemplos, o primeiro é um sistema de molas submetido a carregamentos externos; o segundo é uma placa quadrada modelada com elementos finitos com o módulo de elasticidade sendo modelado como um campo estocástico; o terceiro exemplo trata da resposta obtida para equação de estado limite não linear, onde a concavidade é estudada como influência na aplicação da Amostragem por Importância nas variações aqui discutidas.

Bucher (2009), no artigo intitulado Asymptotic sampling for high dimensional reliability analysis, apresenta um procedimento para análise de confiabilidade como alternativa de solução em problemas com grande número de variáveis aleatórias, tal procedimento foi chamado de Amostragem Assintótica. Além disso, foi dada ênfase aos processos aleatórios e campos aleatórios onde diversos exemplos são tratados. A ideia básica por trás da técnica é parametrizar os desvios padrão das variáveis aleatórias do problema, a fim de se obter mais amostras dentro do domínio de falha e então realizar um ajuste não linear aos dados formados pelo parâmetro $f$ e pelo índice de confiabilidade $\beta$.

Sichani, Nielsen e Bucher (2011), no artigo Applications of asymptotic sampling on high dimensional structural dynamics problems, apresentam diversas aplicações da amostragem assintótica em vários modelos estruturais sujeitos à excitação aleatória. Os autores também apresentam uma melhor análise da dependência do desempenho da técnica, com os parâmetros que podem ser calibrados. Com o foco em problemas de dinâmica estrutural, especialmente envolvendo vibrações aleatórias, é apresentada uma técnica que calibra o intervalo adotado para os pontos de suporte e assim é possível obter um menor coeficiente de variação para a probabilidade de falha estimada. Vale ressaltar que a aplicação do algoritmo de otimização, para a calibração do intervalo dos pontos de suporte, apresenta uma boa aplicação em problemas envolvendo vibrações aleatórias.

Naess, Leira e Batsevych (2009), no artigo intitulado System reliability analysis by enhanced Monte Carlo simulation, apresentam uma técnica para estimativa eficiente da probabilidade de falha de sistemas. Tal técnica é baseada na parametrização da função de 
estado limite, onde através de um procedimento de regressão não linear o valor da probabilidade de falha pode ser obtido por extrapolação.

Au e Beck (2001), no artigo Estimation of small failure probabilities in high dimensions by subset simulation, propõem um método para estimativa de pequenas probabilidades de falha, o método é chamado de Simulação de Subconjunto. A ideia do método é expressar a probabilidade de falha como um produto de probabilidades de falha condicionais, que são probabilidades maiores devido à escolha adequada dos eventos condicionais (subconjuntos). Dessa forma, em cada subconjunto é utilizada uma amostra pequena. O objetivo é suprir as deficiências do método de Monte Carlo Bruto com Amostragem Simples, que apresenta uma baixa eficiência na estimativa de probabilidades de falha pequenas. Na determinação das probabilidades de falhas condicionais é adotada a técnica de simulação de Monte Carlo com cadeias de Markov utilizando o algoritmo de Metropolis-Hastings modificado.

$\mathrm{Au}$, Ching e Beck (2007), apresentam no artigo Application of subset simulation methods to reliability benchmark problems, um estudo comparativo de técnicas que são variações da simulação de subconjuntos, onde três problemas são analisados. O primeiro é uma barragem de terra com variação aleatória das propriedades do solo; o segundo problema é um oscilador do tipo Duffing, com vários graus de liberdade e o terceiro é um edifício em cisalhamento com vários graus de liberdade. As técnicas comparadas foram o método de Monte Carlo Bruto com Amostragem Simples, Simulação de Subconjuntos, Simulação de Subconjunto/Splitting e Simulação de Subconjunto/Hibrido.

Schuëller e Pradlwarter (2007), no artigo Benchmark study on reliability estimation in higher dimensions of structural systems - An overview, fazem uma coletânea de diversos resultados apresentados na literatura sobre procedimentos alternativos adotados em confiabilidade de estruturas com respeito a sua eficiência numérica e computacional. A ênfase do estudo foi em sistemas que incluem um grande número de variáveis aleatórias, onde os problemas estudados por Au, Ching e Beck (2007) são adotados no trabalho em questão. As técnicas comparadas foram as apresentadas por Au, Ching e Beck (2007), com o acréscimo das técnicas de Simulação de Subconjunto Esférica, do Método do Domínio Auxiliar, da Amostragem em Linha, da representação aproximada da função de performance e da redução de modelo para representação por polinômios de caos. 
Como a maioria das técnicas apresentadas possui um desenvolvimento recente, diversos aprimoramentos estão sendo apresentados por diversos autores. Desta forma, este trabalho se concentrará nos conceitos fundamentais de cada uma. 


\section{CONCEITOS DE PROBABILIDADE E ESTATÍSTICA}

Segundo Ang e Tang (1984), muitos processos ou fenômenos aplicados à engenharia contém aleatoriedade, sendo os reais resultados, em algum grau, imprevisíveis. Tais fenômenos são caracterizados por observações experimentais que são invariavelmente diferentes de outro experimento, mesmo que realizados sob as mesmas circunstâncias.

A engenharia moderna encara a incerteza como inerente à natureza do problema que se está analisando. Assim, o estudo da teoria de probabilidades é parte integrante e fundamental da teoria da confiabilidade e na aplicação do método de Monte Carlo na análise de confiabilidade de estruturas.

Este capítulo procura dar uma abordagem aos principais tópicos dentro da teoria de probabilidades, que é de grande importância no desenvolvimento das técnicas a serem estudadas. A seguir serão apresentados alguns conceitos importantes na fundamentação deste trabalho.

\subsection{Axiomas da teoria de probabilidades}

Seja $A$ um evento qualquer, a probabilidade de ocorrência do evento $A$ pode ser apresentada de três formas diferentes. A definição em frequência diz que:

$$
P[A]=\lim _{n \rightarrow \infty} \frac{n_{A}}{n}
$$

onde $n_{A}$ é o número de resultados observados, que levaram à ocorrência do evento $A$, e $n$ é o número total de observações. Tal definição é dita ser a posteriori, pois $P[A]$ só é conhecida após todas as realizações do evento $A$ terem ocorrido. Na definição clássica $P[A]$ é conhecida antes da primeira realização do evento $A$, assim a definição clássica pode ser escrita como:

$$
P[A]=\frac{N_{A}}{N}
$$

onde $N_{A}$ é o número de resultados possíveis, que levam à ocorrência do evento $A$, e $N$ é o número total de resultados. A definição clássica é também conhecida como a priori. 
A definição axiomática é dotada de um maior rigor matemático, que é definido pela teoria de probabilidades. Ela diz que a probabilidade é atribuída a eventos por uma função de distribuição no espaço amostral $\Omega$ e satisfaz as seguintes propriedades:

$$
\begin{array}{cl}
\text { I. } & P[E] \geq 0 ; \\
\text { II. } & P[\Omega]=1 ; \\
\text { III. } & \text { Se } E \subset F \subset \Omega, \text { então } P[E] \leq P[F] ; \\
\text { IV. } & P[A \cup B]=P[A]+P[B] \text { se } A \text { e } B \text { são conjuntos disjuntos de } \Omega ; \\
\text { V. } & P[\bar{A}]=1-P[A] \forall A \subset \Omega .
\end{array}
$$

Em se tratando de simulação, a determinação da probabilidade de ocorrência de um evento de interesse pode ser obtida através da repetição do experimento em um grande número de vezes, através da geração de números aleatórios. Essa é uma tarefa natural com o uso de computadores.

\subsection{Probabilidades condicionais e independência de eventos}

A probabilidade de ocorrência de um evento $A$ condicionado à ocorrência de um evento $B$, tal que $P[B]>0$, é dada por:

$$
P[A \mid B]=\frac{P[A \cap B]}{P[B]}
$$

A definição da probabilidade condicional nos leva a determinação de algumas propriedades na relação entre dois eventos $A$ e $B$, de tal forma que se os eventos $A$ e $B$ são mutuamente excludentes, então:

$$
P[A \cap B]=0 \therefore P[A \mid B]=0 .
$$

Se $A \subset B$, então $A \cap B=A$, então:

$$
P[A \mid B]=\frac{P[A]}{P[B]} \geq P[A] .
$$

Caso $B \subset A$, então $A \cap B=B$, então:

$$
P[A \mid B]=\frac{P[B]}{P[B]}=1 .
$$

Montgomery e Runger (2003) dizem que dois eventos $A$ e $B$ são independentes se alguma das afirmações abaixo for verdade. 


$$
\begin{gathered}
P[A \mid B]=P[A], \\
P[B \mid A]=P[B], \\
P[A \cap B]=P[A] \cdot P[B] .
\end{gathered}
$$

Estendendo essa definição para um número maior de eventos, tem-se que, os eventos $E_{1}, E_{2}, \ldots, E_{n}$ são independentes se e somente se para algum subconjunto desses eventos, $E_{i 1}, E_{i 2}, \ldots, E_{i k}$,

$$
P\left[E_{i 1} \cap E_{i 2} \cap \ldots \cap E_{i k}\right]=P\left[E_{i 1}\right] \times P\left[E_{i 2}\right] \times \ldots \times P\left[E_{i k}\right] .
$$

\subsection{Variáveis aleatórias}

Uma variável aleatória pode assumir um valor real, ou pode assumir qualquer valor definido por um intervalo, tal que o evento $\{X=x\}$ indica que a variável aleatória $X$ assume o valor $x$. Já o evento $\{X \leq x\}$ indica que a variável aleatória $X$ assume qualquer valor menor ou igual a $x$. Como se pode observar, a variável aleatória é definida por uma letra maiúscula e a realização desta por uma letra minúscula. A depender dos elementos que formam o espaço amostral $\Omega$, a variável aleatória pode ser do tipo discreta, quando $\Omega$ é formado por um número finito ou infinito contável de pontos, ou do tipo contínua, quando $\Omega$ é formado por um número infinito de pontos. Matematicamente, pode-se dizer que uma variável aleatória real $X(\omega)$ é uma função real que atribui a cada ponto amostral $\omega$ de um espaço amostral $\Omega$, um valor real $x$, tal que o conjunto $\{X<x\}$ é um evento para qualquer número real $x$. Neste caso observase que o espaço amostral $\Omega$ é o domínio de $X(\omega)$ (BECK, 2012).

\subsubsection{Função de distribuição acumulada de probabilidade}

Muitas vezes estamos interessados em conhecer a probabilidade de ocorrência do evento definido por $\{X \leq x\}$; logo, como tal probabilidade depende apenas de $x$, isso nos leva a existência de uma função $F_{X}(x)=P[\{X \leq x\}]$. Tal função é conhecida como função de distribuição acumulada de probabilidades (FDA) da variável aleatória $X$, e é definida no intervalo $-\infty \leq x \leq+\infty$.

Montgomery e Runger (2003) definem a função de distribuição acumulada de uma variável aleatória $X$ como: 


$$
F_{X}(x)=P[\{X \leq x\}]=\int_{-\infty}^{x} f_{X}(x) d x
$$

onde $f_{X}(x)$ é a função densidade de probabilidade da variável aleatória $X$, que será vista a seguir.

\subsubsection{Função densidade de probabilidade}

A derivada da função de distribuição acumulada de probabilidade da variável aleatória $X$ define a função densidade de probabilidade (FDP) de $X$, que é $f_{X}(x)$, tal que:

$$
f_{X}(x)=\frac{d F_{X}(x)}{d x}
$$

Tal função é usada para descrever uma variável aleatória, podendo ser ela contínua ou discreta. Na literatura, diversos modelos de função de densidade de probabilidades são apresentados, assim como suas aplicações. Dessa forma, fenômenos como a ocorrência de chuvas, de ventos, de estados de mar, de resistência dos materiais entre outros, podem ser modelados com tais funções.

Montegomery e Runger (2003) dizem que a função densidade de probabilidade $f_{X}(x)$ pode ser utilizada na definição da distribuição de probabilidade de uma variável aleatória contínua. Assim, são definidas as seguintes propriedades:

$$
\begin{gathered}
f_{X}(x) \geq 0 \\
\int_{-\infty}^{\infty} f_{X}(x) d x=1, \\
P[\{a \leq X \leq b\}]=\int_{a}^{b} f_{X}(x) d x .
\end{gathered}
$$

\subsubsection{Momentos de uma variável aleatória}

Conhecendo-se a função de densidade de probabilidades da variável aleatória $X$, é possível definir algumas quantidades que caracterizam a variável aleatória. Tais quantidades são chamadas de momentos.

Tem-se que o momento de ordem $k$ para uma variável aleatória continua é dado por: 


$$
E\left[X^{k}\right]=\mu^{k}=\int_{-\infty}^{+\infty} x^{k} f_{X}(x) d x
$$

Um caso particular desta generalização é o valor esperado de $X$, que é um momento de primeira ordem, tal que:

$$
E[X]=\mu=\int_{-\infty}^{+\infty} x f_{X}(x) d x
$$

onde $E[$.$] é o operador valor esperado e \mu$ é o valor do momento de primeira ordem da variável $x$ e que caracteriza sua média.

Os momentos centrais de ordem $k$ para variáveis aleatórias contínuas são calculados em relação à média $\mu$, da seguinte forma:

$$
E\left[(X-\mu)^{k}\right]=m^{k}=\int_{-\infty}^{+\infty}(x-\mu)^{k} f_{X}(x) d x
$$

O momento central de segunda ordem é a variância, que é obtida da seguinte forma:

$$
E\left[(X-\mu)^{2}\right]=m^{2}=\int_{-\infty}^{+\infty}(x-\mu)^{2} f_{X}(x) d x
$$

Pode-se escrever a variância como:

$$
\operatorname{Var}[X]=E\left[(X-\mu)^{2}\right]=\sigma^{2},
$$

de tal forma que $\sigma=\sqrt{\operatorname{Var}[X]}$ é o desvio-padrão da variável aleatória e é uma medida que dá a ideia de dispersão de $X$ em torno da sua média $\mu$.

\subsubsection{Média e variância de amostras}

Se for retirada uma amostra de tamanho $n$ de uma população com média $\mu$ e variância $\sigma^{2}$, então a média $\bar{\mu}$ e a variância $\bar{\sigma}^{2}$ desta amostra podem ser determinadas por:

$$
\begin{gathered}
\bar{\mu}=\frac{1}{n} \sum_{i=1}^{n} x_{i}, \\
\bar{\sigma}^{2}=\frac{1}{n-1} \sum_{i=1}^{n}\left(x_{i}-\bar{\mu}\right)^{2} .
\end{gathered}
$$


Neste caso observa-se que a variância apresenta em sua equação uma divisão pelo termo $n-1$. Isso por que a variância está sendo medida pela distância dos pontos $x_{i}$ em relação à média da amostra $\bar{\mu}$, e não em relação à média populacional $\mu$, então para corrigir tal tendenciosidade a Eq. (23) é utilizada.

\subsection{Distribuição conjunta de probabilidades}

Sejam as variáveis aleatórias $X_{i}$, com $i=1,2, \ldots, n$. Os conjuntos $\left\{X_{i} \leq x_{i}\right\}$ formam eventos cujas probabilidades são dadas por:

$$
P\left[\left\{X_{i} \leq x_{i}\right\}\right]=F_{X_{i}}\left(x_{i}\right)
$$

Então tomando o produto dos $\left\{X_{i} \leq x_{i}\right\}$, pode-se formar o evento conjunto, tal que:

$$
\begin{gathered}
\left\{X_{1} \leq x_{1}\right\}\left\{X_{2} \leq x_{2}\right\} \ldots\left\{X_{i} \leq x_{i}\right\} \ldots\left\{X_{n} \leq x_{n}\right\}= \\
\left\{X_{1} \leq x_{1} ; X_{2} \leq x_{2} ; \ldots ; X_{i} \leq x_{i} ; \ldots ; X_{n} \leq x_{n}\right\}
\end{gathered}
$$

Logo, a probabilidade de ocorrência deste evento leva a definição da distribuição conjunta cumulativa de probabilidades, tal que:

$$
\begin{gathered}
F_{X_{1} X_{2} \ldots X_{i} \ldots X_{n}}\left(x_{1}, x_{2}, \ldots, x_{i}, \ldots, x_{n}\right)= \\
P\left[\left\{X_{1} \leq x_{1} ; X_{2} \leq x_{2} ; \ldots ; X_{i} \leq x_{i} ; \ldots ; X_{n} \leq x_{n}\right\}\right]
\end{gathered}
$$

onde as funções $F_{X_{i}}\left(x_{i}\right)$ são chamadas de distribuições marginais de probabilidade. A função conjunta de densidade de probabilidade $f_{X_{1} X_{2} \ldots X_{i} \ldots X_{n}}\left(x_{1}, x_{2}, \ldots, x_{i}, \ldots, x_{n}\right)$ pode ser obtida da função de distribuição conjunta cumulativa de probabilidade $F_{X_{1} X_{2} \ldots X_{i} \ldots X_{n}}\left(x_{1}, x_{2}, \ldots, x_{i}, \ldots, x_{n}\right)$, desde que essa função possua derivadas parciais de segunda ordem, de tal forma que:

$$
f_{X_{1} X_{2} \ldots X_{i} \ldots X_{n}}\left(x_{1}, x_{2}, \ldots, x_{i}, \ldots, x_{n}\right)=\frac{\partial^{n} F_{X_{1} X_{2} \ldots X_{i} \ldots X_{n}}\left(x_{1}, x_{2}, \ldots, x_{i}, \ldots, x_{n}\right)}{\partial x_{1} \partial x_{2} \ldots \partial x_{i} \ldots \partial x_{n}}
$$

Sabe-se que $F_{X_{1} X_{2} \ldots X_{i} \ldots X_{n}}\left(x_{1}, x_{2}, \ldots, x_{i}, \ldots, x_{n}\right)$ geralmente não pode ser obtida diretamente das funções de distribuição marginal de probabilidade, pois tal resultado muda caso as variáveis sejam correlacionadas ou não, porém, as funções de distribuição marginais de probabilidade podem ser obtidas a partir da distribuição conjunta cumulativa de probabilidade, através de procedimentos de integração, tal que: 


$$
\begin{gathered}
F_{X_{i}}\left(x_{i}\right)= \\
\int_{-\infty}^{+\infty} \int_{-\infty}^{+\infty} \ldots \int_{-\infty}^{x_{i}} \ldots \int_{-\infty}^{+\infty} f_{X_{1} X_{2} \ldots X_{i} \ldots X_{n}}\left(u_{1}, u_{2}, \ldots, u_{i}, \ldots, u_{n}\right) d u_{1}, d u_{2}, \ldots, d u_{i}, \ldots, d u_{n}
\end{gathered}
$$

Considerando que se deseja calcular a probabilidade do evento $\left\{\left(x_{1}, x_{2}, \ldots, x_{n}\right) \in D\right\}$, onde $D$ é uma região do hiperplano $x_{1} \times x_{2} \times \ldots \times x_{n}$, assim tal probabilidade pode ser avaliada por:

$$
P\left[\left\{\left(x_{1}, x_{2}, \ldots, x_{n}\right) \in D\right\}\right]=\int \ldots \int_{D} f_{X_{1} X_{2} \ldots X_{n}}\left(x_{1}, x_{2}, \ldots, x_{n}\right) d x_{1}, d x_{2}, \ldots, d x_{n}
$$

\subsubsection{Momentos conjuntos entre variáveis aleatórias}

Como visto na seção 3.3.3, podem ser definidas algumas quantidades, chamadas de momentos, que caracterizam uma variável aleatória. Conhecida a função conjunta de densidade de probabilidade, podem-se definir os momentos e os momentos centrais destas variáveis aleatórias, de forma que os momentos de ordem arbitrária são definidos por:

$$
\begin{aligned}
& \mu^{k_{1} k_{2} \ldots k_{n}}=E\left[X_{1}{ }^{k_{1}} X_{2}{ }^{k_{2}} \ldots X_{n}{ }^{k_{n}}\right] \\
& =\int_{-\infty}^{+\infty} \int_{-\infty}^{+\infty} \ldots \int_{-\infty}^{+\infty} x_{1}{ }^{k_{1}} x_{2}{ }^{k_{2}} \ldots x_{n}{ }^{k_{n}} f_{X_{1} X_{2} \ldots X_{n}}\left(x_{1}, x_{2}, \ldots, x_{n}\right) d x_{1}, d x_{2}, \ldots, d x_{n}
\end{aligned}
$$

Os momentos centrais são dados em relação à média da respectiva variável aleatória, tal que:

$$
\begin{aligned}
m^{k_{1} \ldots k_{n}}=E\left[\left(X_{1}-\mu_{X_{1}}\right)^{k_{1}} \ldots\left(X_{n}-\mu_{X_{n}}\right)^{k_{n}}\right] \\
=\int_{-\infty}^{+\infty} \ldots \int_{-\infty}^{+\infty}\left(x_{1}-\mu_{X_{1}}\right)^{k_{1}} \ldots\left(x_{n}\right. \\
\left.-\mu_{X_{n}}\right)^{k_{n}} f_{X_{1} \ldots X_{n}}\left(x_{1}, \ldots, x_{n}\right) d x_{1}, \ldots, d x_{n} .
\end{aligned}
$$

A ordem do momento conjunto é dada pela soma dos termos $k_{i}$. Assim, o momento $\mu^{00 \ldots k_{i} \cdots 0}$, com $k_{i}=2$, corresponde ao momento de ordem dois da variável $X_{i}$ e $m^{00 \ldots k_{i} \ldots 0}$, 
com $k_{i}=2$, corresponde ao momento central de ordem 2 da variável $X_{i}$, ou seja, a variância desta.

A covariância é uma medida linear da relação entre duas variáveis aleatórias no mesmo espaço de probabilidade. Tal quantidade pode ser obtida quando $k_{i}=1$ e $k_{j}=1$ com $i \neq j$. Assim, para duas variáveis aleatórias $X$ e $Y$ pode-se escrever a covariância como:

$$
\operatorname{Cov}[X, Y]=E\left[\left(X-\mu_{X}\right)\left(Y-\mu_{Y}\right)\right]
$$

Uma medida adimensional que deriva da covariância entre duas variáveis é o coeficiente de correlação de Pearson, tal que:

$$
\rho_{X Y}=\frac{\operatorname{Cov}[X, Y]}{\sigma_{X} \sigma_{Y}}
$$

Tal valor varia entre -1 e 1 , tal que $\rho_{X Y}=1$ indica uma correlação perfeita positiva e $\rho_{X Y}=$ -1 indica uma correlação perfeita negativa, porém $\rho_{X Y}=0$ não necessariamente indica dependência nula (BECK, 2012). 


\section{CONFIABILIDADE DE ESTRUTURAS}

Neste Capítulo serão apresentados o conceito de estados limites e os requisitos que uma estrutura deve atender, para que posteriormente seja introduzido o problema fundamental da confiabilidade, seguindo do método de confiabilidade de primeira ordem e por fim da confiabilidade de sistemas.

\subsection{Estados limites}

Estruturas são dimensionadas de forma a obedecer alguns requisitos básicos que garantam um bom desempenho durante a vida útil das mesmas. Segundo Beck (2012), uma estrutura deve cumprir uma determinada função estrutural durante a sua vida útil, com um nível adequado de segurança e de maneira economicamente viável. Os requisitos básicos são: o de serviço, de segurança, de robustez, econômico e social. O requisito de serviço diz que uma estrutura deve manter-se em condições apropriadas para o uso adequado durante sua vida útil. O requisito de segurança diz que uma estrutura deve suportar carregamentos extremos, esporádicos e carregamentos repetitivos, dentro de sua vida útil e sem que a estrutura entre em colapso. O requisito de robustez diz que uma estrutura não deve ser danificada de maneira desproporcional à severidade do evento causador do dano durante sua vida útil. $\mathrm{O}$ requisito econômico diz que uma estrutura deve, obedecendo todos os requisitos apresentados anteriormente, ser economicamente viável. O requisito social qualifica a aceitação da sociedade ao nível de segurança, definido pelos requisitos citados anteriormente, da estrutura em questão.

Os modos de falha de uma estrutura, que são as distintas maneiras que levam a um estado indesejável, dão origem aos estados limites. Os estados limites podem ser de serviço ou último, e englobam os requisitos apresentados anteriormente. O estado limite de serviço é caracterizado por danos, deformações, vibrações e outras condições que, mantendo as condições de serviço, possam levar ao desconforto do usuário. Já o estado limite último é caracterizado pela inadequação de uso da estrutura após esta atingir tal estado, tendo ela entrado em colapso ou não, pois a situação corresponde a um estado inseguro para uso da mesma.

Os requisitos que uma estrutura deve obedecer durante sua vida útil podem ser equacionados através de equações de estado limite. Estas equações caracterizam os distintos 
modos de falha de uma estrutura, onde para cada modo de falha $i$ se escreve uma função $g_{i}(\mathbf{X})$, que é função do vetor de variáveis aleatórias $\mathbf{X}=\left[X_{1}, X_{2}, \ldots, X_{n}\right]$, de tal forma que o estado limite fica determinado pela seguinte igualdade:

$$
g_{i}(\mathbf{X})=g_{i}\left(X_{1}, X_{2}, \ldots, X_{n}\right)=0
$$

Uma equação de estado limite $g_{i}(\mathbf{X})$ é escrita de forma a dividir o domínio do problema em domínios de falha $\Omega_{\mathrm{f}}$ e de segurança $\Omega_{\mathrm{s}}$, tal que:

$$
\begin{aligned}
& \Omega_{\mathrm{f}}=\left\{\mathbf{x} \mid g_{i}(\mathbf{x}) \leq 0\right\}, \\
& \Omega_{\mathrm{s}}=\left\{\mathbf{x} \mid g_{i}(\mathbf{x})>0\right\} .
\end{aligned}
$$

A Figura 1 apresenta graficamente a fronteira entre o domínio de falha e o domínio de segurança para o espaço amostral de duas variáveis aleatórias.

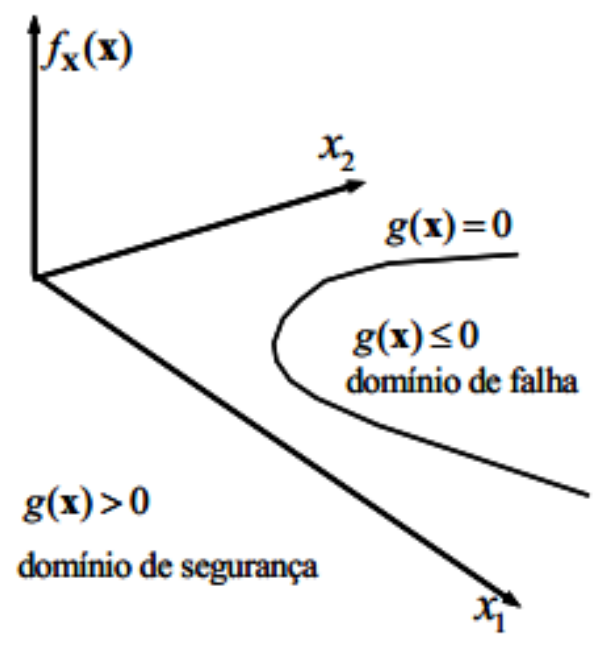

Figura 1. Equação de estado limite e domínios de falha e segurança (BECK, 2012).

A formulação apresentada acima se aplica a problemas independentes do tempo. Quando se deseja resolver problemas dependentes do tempo, os domínios de falha e segurança ficam definidos por:

$$
\begin{aligned}
& \Omega_{\mathrm{f}}=\left\{\mathbf{x} \mid g_{i}(\mathbf{x}, t) \leq 0\right\}, \\
& \Omega_{\mathrm{s}}=\left\{\mathbf{x} \mid g_{i}(\mathbf{x}, t)>0\right\} .
\end{aligned}
$$

A utilização da variável tempo pode ser consequência de uma dependência temporal do estado limite ou através da consideração de carregamentos definidos por processos estocásticos (BECK, 2012). 


\subsection{Problema fundamental da confiabilidade de estruturas}

A avaliação da probabilidade de ocorrência de eventos indesejáveis nos leva a definição de probabilidade de falha $P_{f}$, onde se pode escrever:

$$
P_{f}=P\left[\mathbf{X} \in \Omega_{\mathrm{f}}\right]=P[\{g(\mathbf{X})<0\}] .
$$

Desta forma, diz-se que a probabilidade de falha $P_{f}$ é uma medida de violação de estados limites. Considerando um caso do tipo resistência-solicitação $(R-S)$, temos que:

$$
g(R, S)=R-S
$$

Assim, a probabilidade de falha é avaliada pela Eq. (39), que é o problema fundamental da confiabilidade de estruturas.

$$
P_{f}=P[\{R-S<0\}]=P\left[(R, S) \in \Omega_{\mathrm{f}}\right]=\iint_{\Omega_{\mathrm{f}}} f_{R S}(r, s) d r d s,
$$

onde $(r, s)$ é a realização da dupla das variáveis aleatórias $R$ e $S$ e $f_{R S}(r, s)$ é a função conjunta de densidade de probabilidade de $R$ e $S$.

De maneira geral temos que a probabilidade de falha pode ser definida por:

$$
P_{f}=P\left[\mathbf{X} \in \Omega_{\mathrm{f}}\right]=\int_{\Omega_{\mathrm{f}}} f_{\mathbf{X}}(\mathbf{x}) d \mathbf{x}
$$

Outra forma de resolver o problema fundamental de confiabilidade é através da integração da função de densidade de probabilidade da função margem de segurança $M$. Sendo $M$ dado por:

$$
M=R-S
$$

Como $R$ e $S$ são variáveis aleatórias, então $M$ será uma variável aleatória com função de densidade de probabilidades dada por $f_{M}(m)$, assim a probabilidade de falha desse problema é dada por:

$$
P_{f}=P[\mathrm{M}<0]=\int_{-\infty}^{0} f_{M}(m) d m
$$




\subsection{Método de confiabilidade de primeira ordem}

Em confiabilidade estrutural é importante conceituar ponto de projeto (design point) e o índice de confiabilidade $\beta$. O ponto de projeto pode ser definido como o ponto no domínio de falha que apresenta a menor distância para a origem do espaço normal padrão. Além disso, tanto no espaço normal padrão, quanto no espaço de projeto, é o ponto que guarda o maior conteúdo de probabilidade, ou seja, é o ponto no domínio de falha com maior probabilidade de ocorrência. Já o índice de confiabilidade $\beta$ possui duas interpretações. A primeira o relaciona com a probabilidade de falha, tal que sendo $R$ e $S$ duas variáveis aleatórias normais não correlacionadas e tomando o problema definido na Eq. (40), a média e o desvio padrão da função margem de segurança ficam definidos por:

$$
\begin{gathered}
\mu_{M}=\mu_{R}-\mu_{S}, \\
\sigma_{M}=\sqrt{\sigma_{R}^{2}+\sigma_{S}^{2}} .
\end{gathered}
$$

Através da transformação de Hassofer-Lind a variável $M$ pode ser transformada em uma variável normal padrão Y, tal que:

$$
\mathrm{Y}=\frac{\mathrm{M}-\mu_{M}}{\sigma_{M}}
$$

A probabilidade associada ao evento falha pode ser definida através da função de distribuição acumulada de probabilidade normal padrão $\Phi($. ). Logo, tem-se que:

$$
P_{f}=\mathrm{P}(\mathrm{M} \leq 0)=\mathrm{P}\left(\mathrm{Y} \leq-\frac{\mu_{M}}{\sigma_{M}}\right)=\Phi\left(-\frac{\mu_{M}}{\sigma_{M}}\right)=\Phi(-\beta)
$$

Assim:

$$
\beta=\frac{\mu_{M}}{\sigma_{M}}=\frac{\mu_{R}-\mu_{M}}{\sqrt{\sigma_{R}^{2}+\sigma_{S}^{2}}}
$$

Também se pode atribuir uma interpretação geométrica para o índice de confiabilidade. Tomando a mesma equação de estado limite e realizando cálculos de minimização da distância, em duas dimensões, do ponto de projeto à origem do espaço normal padrão, se consegue obter a seguinte relação: 


$$
d_{\min }=\frac{\mu_{R}-\mu_{M}}{\sqrt{\sigma_{R}^{2}+\sigma_{S}^{2}}}
$$

Esta equação é equivalente à apresentada na Eq. (49). Dessa forma, pode definir o índice de confiabilidade como uma medida geométrica da probabilidade de falha que corresponde a menor distância entre a equação de estado limite e a origem do espaço normal padrão.

O FORM e o SORM são métodos de transformação utilizados em confiabilidade. O FORM (First Order Reliability Method) é o método de confiabilidade de primeira ordem. Este utiliza toda informação estatística em relação às variáveis do problema, o que inclui variáveis com distribuição marginal não gaussiana e coeficientes de correlação entre pares de variáveis. Porém, a equação de estado limite é aproximada por um hiperplano no ponto de projeto. O SORM (Second Order Reliability Method) é o método de confiabilidade de segunda ordem. Este utiliza as mesmas informações adotadas para o FORM, porém, a equação de estado limite é aproximada por uma hipersuperfície quadrática no ponto de projeto. O FORM é geralmente aceito como uma forma eficiente de resolver o problema na Eq. (39), para pequenas ou moderadas dimensões do vetor $\mathbf{X}$ e para equações de estado limites $g(\mathbf{x})$ lineares ou fracamente não lineares.

O FORM se baseia na construção da função conjunta de probabilidade $f_{\mathbf{X}}(\boldsymbol{x})$ e sua transformação para o espaço normal padrão. Tal construção envolve a eliminação de correlação entre variáveis aleatórias e o cálculo das variáveis normais equivalentes. A transformação citada corresponde a um mapeamento indireto do vetor $\mathbf{X} \in \mathbb{X}$ para o espaço normal padrão $\mathbb{Y}$ :

$$
\mathrm{Y}=\mathbb{T}[\mathrm{X}], \quad g_{\mathrm{Y}}(\mathrm{Y}, t)=g\left(\mathrm{~T}^{-1}[\mathrm{Y}], t\right) .
$$

Para se construir a função conjunta de probabilidades no espaço de projeto $\mathbb{X}$, comumente se tem conhecimento de duas propriedades apenas, que são as distribuições de probabilidades marginais $f_{\mathrm{X}_{i}}\left(x_{i}\right)$ e a matriz de correlação $\mathbf{R}_{\mathbf{X}}$ entre pares de variáveis, tal que:

$$
\mathbf{R}_{\mathbf{X}}=\left[\begin{array}{cccc}
\rho_{\mathrm{X}_{11}} & \rho_{\mathrm{X}_{12}} & \ldots & \rho_{\mathrm{X}_{1 n}} \\
\rho_{\mathrm{X}_{21}} & \rho_{\mathrm{X}_{22}} & \ldots & \rho_{\mathrm{X}_{2 n}} \\
\vdots & \vdots & \ddots & \vdots \\
\rho_{\mathrm{X}_{n 1}} & \rho_{\mathrm{X}_{n 2}} & \cdots & \rho_{\mathrm{X}_{n n}}
\end{array}\right]
$$

$\mathrm{Na}$ Eq. (52) tem-se que $n$ é o número de variáveis aleatórias e $\rho_{\mathrm{X}_{i i}}=1$, com $i=1,2, \ldots, n$ (BECK, 2012). 


\subsubsection{Transformação composta utilizando o modelo de Nataf}

O mapeamento realizado de $\mathbb{X} \rightarrow \mathbb{Y}$ é dito indireto, pois se utiliza uma transformação da distribuição conjunta de probabilidade das variáveis $\mathbf{X} \in \mathbb{X}$ em uma distribuição conjunta de variáveis normais equivalentes $\mathbf{Z} \in \mathbb{Z}$, onde $\mathbb{Z}$ é o espaço de probabilidade das variáveis aleatórias normais equivalentes que mantém possíveis correlações entre pares de variáveis aleatórias. Tal transformação é baseada no princípio da aproximação normal (DITLEVSEN, 1981). Este princípio consiste em determinar, para um ponto $x_{i}^{*}$, uma distribuição normal equivalente que preserve o conteúdo de probabilidade da distribuição original. Dessa forma, é necessário definir os momentos da distribuição normal equivalente, tal que:

$$
\begin{gathered}
\sigma_{X_{i}}^{n e q}=\frac{\phi\left(z_{i}^{*}\right)}{f_{X_{i}}\left(x_{i}^{*}\right)^{\prime}} \\
\mu_{X_{i}}^{n e q}=x_{i}^{*}-z_{i}^{*} \sigma_{X_{i}}^{n e q},
\end{gathered}
$$

onde $\phi$ é a função densidade de probabilidade normal padrão, $\sigma_{X_{i}}^{\text {neq }}$ é o desvio padrão da distribuição normal equivalente e $\mu_{X_{i}}^{n e q}$ é a média da distribuição normal equivalente.

Esta transformação pode ser definida matricialmente a partir da determinação da matriz $D^{\text {neq }}$ e do vetor $M^{\text {neq }}$, tal que:

$$
\begin{aligned}
\boldsymbol{D}^{n e q} & =\left[\begin{array}{cccc}
\sigma_{X_{1}}^{n e q} & 0 & \cdots & 0 \\
0 & \sigma_{X_{2}}^{n e q} & \cdots & 0 \\
\vdots & \vdots & \ddots & \vdots \\
0 & 0 & \cdots & \sigma_{X_{n}}^{n e q}
\end{array}\right] ; \\
\boldsymbol{M}^{n e q} & =\left\{\begin{array}{llll}
\mu_{X_{1}}^{n e q} & \mu_{X_{2}}^{n e q} & \cdots & \mu_{X_{n}}^{n e q}
\end{array}\right\} .
\end{aligned}
$$

Com $D^{n e q}$ obtêm-se as matrizes jacobianas da transformação $\mathbb{X} \rightarrow \mathbb{Z}$ e $\mathbb{Z} \rightarrow \mathbb{X}$, tal que:

$$
\begin{gathered}
\mathrm{J}_{\mathrm{zx}}=\left(D^{n e q}\right)^{-1}, \\
\mathrm{~J}_{\mathrm{xz}}=D^{n e q}
\end{gathered}
$$

Assim as transformações ficam definidas por:

$$
\begin{gathered}
z=\mathrm{J}_{\mathrm{zx}} \cdot\left\{x-M^{n e q}\right\} \\
x=\mathrm{J}_{\mathrm{xz}} \cdot \mathrm{z}+M^{n e q}
\end{gathered}
$$


Por meio do modelo de Nataf, pode-se construir uma aproximação para a função conjunta de densidade de probabilidades $f_{\mathbf{X}}$ a partir da distribuição normal padrão multivariada $\phi_{n}$, com a matriz de correlação $\mathbf{R}_{\mathbf{z}}$ a ser determinada. Assim, $f_{\mathbf{X}}$ é definida como:

$$
f_{\mathbf{X}}=\phi_{n}\left(\mathbf{z}, \mathbf{R}_{\mathbf{z}}\right) \frac{f_{X_{1}}\left(x_{1}\right) f_{X_{2}}\left(x_{2}\right) \ldots f_{X_{n}}\left(x_{n}\right)}{\phi\left(z_{1}\right) \phi\left(z_{2}\right) \ldots \phi\left(z_{n}\right)}
$$

Os coeficientes de correlações equivalentes para as distribuições marginais normais podem ser obtidos de maneira iterativa por meio da seguinte equação:

$$
\rho_{X_{i j}}=\int_{-\infty}^{\infty} \int_{-\infty}^{\infty} z_{i} z_{j} \phi_{2}\left(z_{i}, z_{j}, \rho_{z_{i j}}\right) d z_{i} d z_{j},
$$

onde $\phi_{2}($.$) é a distribuição normal padrão bivariada. Então, conhecido \rho_{X_{i j}}$, pelo método de tentativas pode-se obter $\rho_{Z_{i j}}$.

Posteriormente, elimina-se a correlação entre os pares de variáveis aleatórias levando-as de $\mathbb{Z} \rightarrow \mathbb{Y}$. Tal eliminação pode ser obtida via decomposição ortogonal ou fatoração de Cholesky, da matriz de correlação. A decomposição ortogonal apresenta um alto custo computacional devido ao grande número de operações matriciais que o método exige. Desta forma, quando se tem matrizes de correlação não cheias, a fatoração de Cholesky apresenta vantagem devido ao menor número de operações matriciais.

Sendo $\mathbf{C}_{\mathbf{Y}}$ a matriz de correlação em $\mathbb{Y}$ e $\mathbf{C}_{\mathbf{Z}}$ a matriz de correlação em $\mathbb{Z}$. As matrizes jacobianas das transformações $\mathbb{Y} \rightarrow \mathbb{Z}$ e $\mathbb{Z} \rightarrow \mathbb{Y}$ utilizando a decomposição ortogonal da matriz de correlação são:

$$
\begin{gathered}
\mathbf{J}_{\mathbf{y z}}=\mathbf{A}^{\mathrm{T}}, \\
\mathbf{J}_{\mathbf{z y}}=\left(\mathbf{A}^{\mathrm{T}}\right)^{-\mathbf{1}},
\end{gathered}
$$

tal que

$$
\mathbf{A}=\overline{\mathrm{A}} \boldsymbol{\Lambda}^{-\frac{1}{2}},
$$

onde $\overline{\mathbf{A}}$ é uma matriz ortogonal cujas colunas são os autovetores de $\mathbf{C}_{\mathrm{Z}}$ e $\boldsymbol{\Lambda}$ é a matriz cujos elementos da diagonal principal são as inversas das raízes dos autovalores de $\mathbf{C}_{\mathbf{Z}}$.

Utilizando a decomposição de Cholesky de $\mathbf{C}_{\mathbf{Z}}$, tem-se que: 


$$
\mathbf{C}_{\mathbf{Z}}=\mathbf{L L}^{\mathrm{T}}
$$

Assim, as matrizes jacobianas obtidas via decomposição de Cholesky de $\mathbf{C}_{\mathbf{Z}}$ são (BECK, 2012):

$$
\begin{gathered}
\mathbf{J}_{\mathrm{yz}}=\mathbf{L}^{-\mathbf{1}}, \\
\mathbf{J}_{\mathrm{zy}}=\mathbf{L} .
\end{gathered}
$$

Logo, as transformações ficam definidas por:

$$
\begin{aligned}
& y=\mathrm{J}_{\mathrm{yz}} \cdot z, \\
& z=\mathrm{J}_{\mathrm{zy}} \cdot y .
\end{aligned}
$$

No espaço normal padrão, a função de densidade conjunta $f_{\mathrm{Y}}(\mathrm{Y})$ tem simetria radial, portanto, o ponto $\mathbf{y}^{*}$ sobre a equação de estado limite $g(\mathbf{Y})$ mais próximo da origem é o ponto de projeto. Esta característica permite resolver o problema de confiabilidade como um problema de otimização com restrições, tal que:

$$
\begin{aligned}
& \mathbf{y}^{*}=\arg \min \sqrt{\mathbf{y}^{t} \mathbf{y}}, \\
& \text { sujeito a } g_{\mathbf{y}}(\mathbf{y})=0 .
\end{aligned}
$$

A partir da Eq. (71), tem-se que $\beta=\left\|\mathbf{y}^{*}\right\|=\left(\mathbf{y}^{* t} \cdot \mathbf{y}^{*}\right)^{1 / 2}$ é o chamado índice de confiabilidade de Hassofer-Lind, que vem a ser a distância entre o ponto $\mathbf{y}^{*}$ e a origem do espaço normal padrão. O método FORM consiste em encontrar o ponto de projeto, a partir da solução da Eq. (71), e aproximar a equação de estado limite $g_{\mathbf{y}}(\mathbf{Y})$ neste ponto por um hiperplano. Portanto, a aproximação de primeira ordem para a probabilidade de falha fica:

$$
P_{f}=P\left[g_{\mathbf{y}}(\mathbf{Y}) \leq 0\right] \simeq \Phi(-\beta) \text {. }
$$

onde $\Phi($.$) é a função de distribuição acumulada de probabilidades normal padrão (BECK,$ 2012).

Dentro do método de Monte Carlo, o FORM apresenta bastante utilidade quando se deseja encontra o ponto de projeto para aplicação de Amostragem por Importância utilizando Pontos de Projetos. 


\subsection{Confiabilidade de sistemas}

A confiabilidade estrutural pode ser aplicada a elementos estruturais ou a sistemas estruturais. Este último se caracteriza pela associação de vários elementos estruturais formando um conjunto resistente a esforços. Elementos estruturais, assim como os sistemas estruturais, podem apresentar diversos modos de falha. Elementos estruturais podem apresentar falhas por escoamento localizado, plastificação da seção, ruptura frágil, deformação excessiva, acúmulo de dano, entre outros. Já os sistemas estruturais podem apresentar falha por deflexão excessiva, vibração excessiva, recalque de apoios, movimentos de corpo rígido, colapso progressivo, entre outros. Considerando que o termo "estrutura" engloba tanto sistemas estruturais quanto elementos estruturais, este termo é adotado de forma generalista neste trabalho.

O método FORM se aplica individualmente a cada equação de estado limite de um problema. Quando uma estrutura possui mais de um modo de falha, uma equação de estado limite $g_{i}(\mathbf{x})$ deve ser escrita para cada possível modo de falha. Neste caso, a estimativa de primeira ordem da probabilidade de falha não se aplica, e limites uni-modais ou bimodais devem ser calculados. Alternativamente, pode-se empregar a simulação de Monte Carlo (BECK, 2012).

Em uma estrutura que possui diferentes modos de falha, as equações de estado limite $g_{i}(\mathbf{x})$ aparecem associadas como um sistema em série (se a falha acontece em relação a qualquer um dos modos, ocorre a falha da estrutura). Neste caso, o domínio de falha é definido por:

$$
\Omega_{\mathrm{f}}=\bigcup_{i}\left(g_{i}(\mathbf{x}) \leq 0\right) .
$$

Para sistemas hiperestáticos com redundância, a falha só ocorre se falharem todos os componentes hiperestáticos. Neste caso, tem-se um sistema em paralelo, e o domínio de falha pode ser caracterizado como:

$$
\Omega_{\mathrm{f}}=\bigcap_{j}\left(g_{i}(\mathbf{x}) \leq 0\right) .
$$

Se cada componente de um sistema hiperestático possui múltiplos modos de falha, então se tem uma associação mista de equações de estado limite, como por exemplo: 


$$
\Omega_{\mathrm{f}}=\bigcap_{j}\left(\bigcup_{i}\left(g_{i}(\mathbf{x}) \leq 0\right)_{j}\right) .
$$

Diversas outras formas de associação mista podem ser obtidas através da manipulação correta das associações de união e intersecção dos domínios de falha individuais. 


\section{GERAÇÃO DE NÚMEROS ALEATÓRIOS}

Computadores digitais comuns não possuem ferramentas de software para gerar sequências de números aleatórios. Uma boa alternativa para isso é a utilização de geradores de números pseudoaleatórios. Segundo Viera, Ribeiro e Souza (2004), o propósito dos geradores de números aleatórios é produzir uma sequência de números que aparentem ser gerados aleatoriamente a partir uma distribuição de probabilidade específica. Geradores usuais, que são aqueles que já estão programados em computadores, geram amostras através de algoritmos sequenciais e determinísticos. Tais algoritmos geram amostras independentes e uniformemente distribuídas no intervalo fechado de $[0,1]$. A sequência gerada pelo algoritmo é determinada pela semente do gerador, de forma que um gerador iniciado com a mesma semente, em máquinas diferentes, deverá gerar a mesma sequência, portanto, pode-se dizer que se está gerando uma sequência de números pseudoaleatórios.

\subsection{Geradores de números aleatórios}

L'Ecuyer (1997) diz que os geradores de número aleatórios possuem um estado em um espaço de estado finito $S$. De acordo com a fórmula de recorrência $s_{n}=f\left(s_{n-1}\right)$ com $n \geq 1$, onde o estado inicial $s_{0}$ é chamado de semente e $f: S \rightarrow S$ é a função de transição. No passo $n$ a função definida por $u_{n}=g\left(s_{n}\right) \mid g: S \rightarrow[0,1]$ é a função de saída, dessa forma a sequência do gerador de números aleatórios fica determinada por $\left\{u_{n} \mid n \geq 0\right\}$, que são variáveis ditas pseudoaleatórias, produzidas pelo gerador.

Sabe-se que $S$ é finito, assim é possível que o gerador retorne a algum estado anterior tal que $s_{i+j}=s_{i}$ para algum $i, j>0$, assim pode-se definir uma medida chamada de período $\rho$ que vem a ser o comprimento de um estado $S$ até que ele ocorra novamente, assim, $\rho$ não pode exceder a cardinalidade de $S$. Por isso pode-se definir que tais geradores geram na realidade uma sequência de números pseudoaleatórios, porém, sem perda de generalidade os geradores estudados neste trabalho serão chamados de geradores de números aleatórios.

Para L'Ecuyer (1997), um longo período $\rho$ e uma boa estrutura de pontos não são as únicas qualidades requeridas para um bom gerador de números aleatórios. Estes precisam ser rápidos, pois, simulações podem exigir a geração de uma quantidade cada vez maior de números aleatórios; precisam ser portáveis, o que significa que o gerador, quando programado 
em diferentes computadores e fazendo uso de diferentes compiladores, deve produzir a mesma sequência; além disso, deve apresentar reprodutibilidade, ou seja, o gerador deve reproduzir a mesma sequência de números aleatórios sempre que for possível. Essa última característica é importante quando se está verificando respostas de programa e em testes de redução de variância, tal característica pode ser considerada uma vantagem em relação a geradores de números aleatórios reais. $\mathrm{O}$ salto à frente (jumping ahead) também é uma importante característica. O salto à frente é habilidade de computar, dado o atual estado $s_{n}, \mathrm{o}$ estado $s_{n+v}$ para algum $v$. A utilidade disto é a quebra da sequência em diversas sequências menores de forma a produzir geradores virtuais.

\subsubsection{Geradores congruentes lineares}

Os geradores congruentes lineares são utilizados como gerador de sequência de números aleatórios no intervalo de $[0,1]$. Tais geradores possuem a seguinte fórmula de recorrência:

$$
x_{n+1}=\left(a x_{n}+c\right) \bmod (m) \text {. }
$$

A Eq. (76) indica que dada semente $x_{0}$ do gerador, uma sequência pode ser obtida pelo resto da divisão entre $\left(a x_{n}+c\right)$ e $(m)$, tal operação é definida pelo operador resto da divisão de $x$ por $y,(x) \bmod (y)$. Este gerador é conhecido como Gerador Congruente Linear Misto. Tal gerador é dito misto, pois envolve as operações de multiplicação e adição por $a$ e $c$ respectivamente. No caso de $c=0$, é dada a denominação de Gerador Congruente Multiplicativo, cuja fórmula de recorrência é:

$$
x_{n+1}=\left(a x_{n}\right) \bmod (m) \text {. }
$$

A qualidade de um gerador congruente linear é dependente das constantes $\{m, a, c\}$. Como os geradores são cíclicos, com período $\rho$ sempre menor que $m$, deve-se adotar um valor grande para $m$ (BECK, 2012). Um gerador congruente linear misto bastante utilizado tem $m=2^{M}$ e $c>0$. O período $\rho$ só é obtido se, e somente se, $a-1$ for múltiplo de 4 e $c$ for ímpar, frequentemente igual a um (VIEIRA;RIBEIRO; SOUZA, 2004). Para se gerar as pontos amostrais uniformemente distribuídos entre 0 e 1 , aplica-se o seguinte procedimento:

$$
u_{i}=\frac{x_{i}}{m^{\prime}}
$$

$\operatorname{com} i=1, \ldots, n$. 
A qualidade de um gerador de números aleatórios pode ser testada pela medida de uniformidade e de independência entre os pontos amostrais gerados, isso é feito via testes estatísticos padronizados. Um teste visual adotado para verificar a uniformidade da amostra é obtido através da distribuição acumulada de probabilidade dos $n$ pontos amostrais gerados, de tal forma que a distribuição acumulada é definida por (BECK, 2012):

$$
F_{U}\left(u_{i}\right)=P\left[\left\{U<u_{i}\right\}\right] \approx \frac{i}{n^{\prime}}
$$

com $i=1, \ldots, n$. Assim, anotando as duplas $\left(i, F_{U}\left(u_{i}\right)\right)$, podem-se gerar os gráficos apresentados na Figura 2 com o gerador congruente linear utilizado nos computadores IBM 360 durante a década de 60. Tal gerador é definido pelos seguintes parâmetros: $\{m, a, c\}=$ $\left\{2^{31}-1,65.539,0\right\}$ e é conhecido como RANDU. Neste trabalho se definirá um gerador pela seguinte notação: $G C L(m, a, c)=\{m, a, c\}$, onde $G C L$ significa Gerador Congruente Linear.

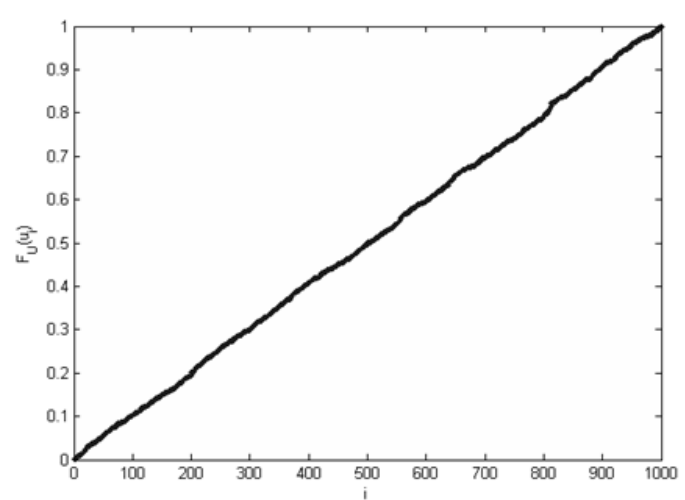

a)

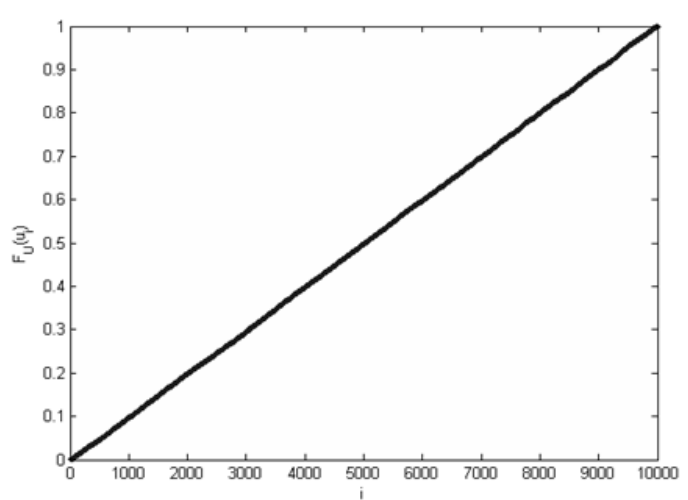

b)

Figura 2. Teste de uniformidade: a) $i=1.000$ e b) $i=10.000$ (à esquerda).

A Figura 2 apresenta um resultado que comprova a aparente uniformidade do gerador RANDU. É observado que a possível uniformidade aumenta quanto mais números aleatórios são gerados, porém, este teste não é considerado robusto. Um teste robusto é o método de partição do hipercubo unitário. A partir deste teste pode-se observar um comportamento uniforme ou a formação de estruturas lattice das $t$-uplas obtidas de números aleatórios consecutivos em duas ou três dimensões. De acordo com Viera, Ribeiro e Souza (2004), considere a sequência $\left\{u_{n} \mid 0 \leq n<\rho\right\}$ no intervalo de $[0,1]$, onde $\rho$ é o período, então se espera que os pares $\left\{\left(u_{n}, u_{n+1}\right) \mid 0 \leq n<\rho\right\}$ cubram todo o espaço bidimensional de maneira uniforme, porém, muitas vezes pode-se observar a formação de linhas de tendências. No caso tridimensional pode-se observar a formação de planos preferenciais. De forma geral, na estrutura lattice todos os pontos adjacentes no espaço $t$-dimensional 
$\left\{\left(u_{n}, u_{n+1}, \ldots, u_{n+t-1}\right) \mid 0 \leq n<\rho\right\}$ estão localizados em uma família de hiperplanos paralelos equidistantes.

As características apresentadas anteriormente podem ser comprovadas de maneira visual. Tal como apresentado por Viera, Ribeiro e Souza (2004), utilizando geradores $\operatorname{GCL}(509,10,0), \operatorname{GCL}(31,3,0)$ e $\operatorname{GCL}(31,13,0)$ com $n=1000$ para todos, pode-se observar o quanto a escolha do parâmetro $a$ interfere na distribuição dos pontos no espaço bidimensional. De acordo com a Figura 3, a Figura 4 e a Figura 5, é possível observar a formação das estruturas lattice de forma que o espaço bidimensional não é completamente preenchido com a distribuição apresentada.

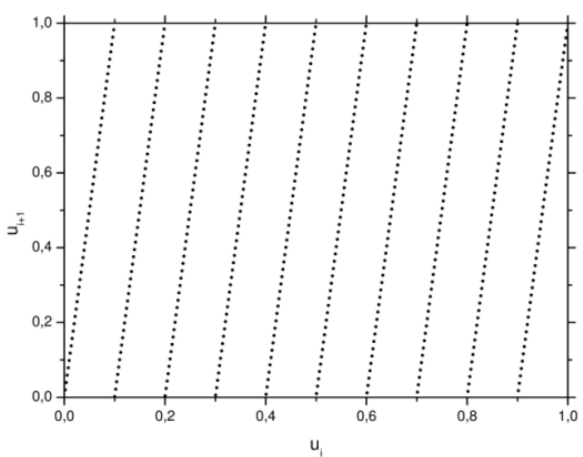

Figura 3. Gerador: $\boldsymbol{G C L}(\mathbf{5 0 9}, \mathbf{1 0}, \mathbf{0})$.

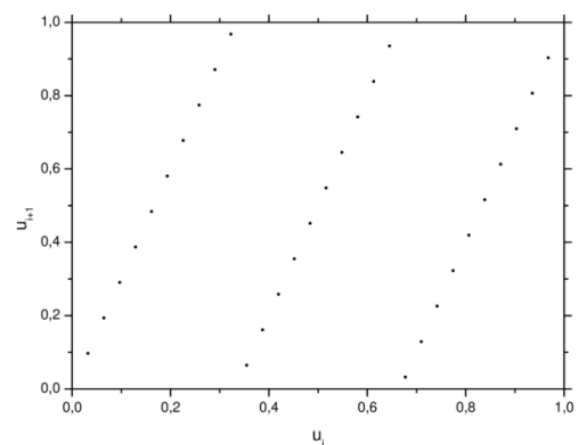

Figura 4. Gerador: $\boldsymbol{G C L}(\mathbf{3 1}, \mathbf{3}, \mathbf{0})$.

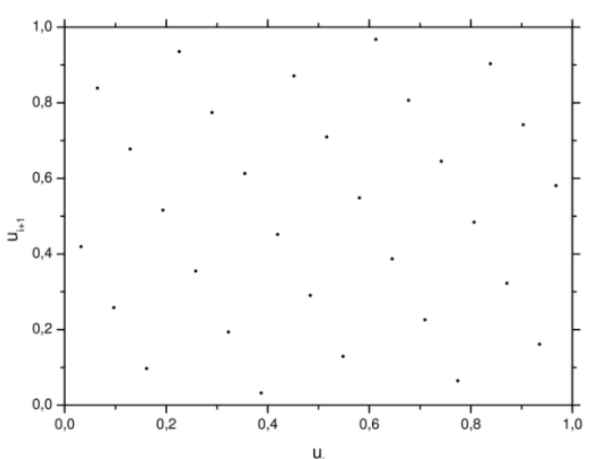

Figura 5. Gerador: $\boldsymbol{G C L}(\mathbf{3 1}, \mathbf{1 3}, \mathbf{0})$. 
Para o gerador RANDU pode-se observar uma distribuição no espaço bidimensional (Figura 6), onde aparentemente apresenta uma boa distribuição dos números aleatórios.

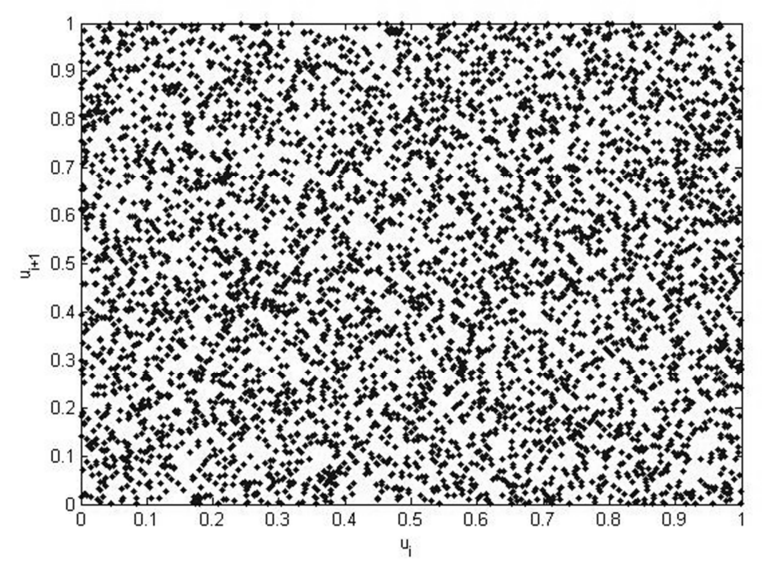

Figura 6. Gerador RANDU: $\operatorname{GCL}\left(\mathbf{2}^{31}-\mathbf{1}, \mathbf{6 5 . 5 3 9}, \mathbf{0}\right)$.

Um método mais robusto é a partição do hipercubo unitário em sua versão tridimensional. Assim, as triplas $\left(u_{i}, u_{i+1}, u_{i+2}\right)$, com $i=1, \ldots, n$, são plotadas e testes estatísticos podem ser utilizados para testar a uniformidade, porém, em alguns casos uma análise visual é suficiente para atestar a uniformidade ou não dos números gerados. Para o gerador RANDU, a Figura 7 apresenta a formação de planos preferenciais, o que indica que o gerador apresenta um problema de uniformidade.

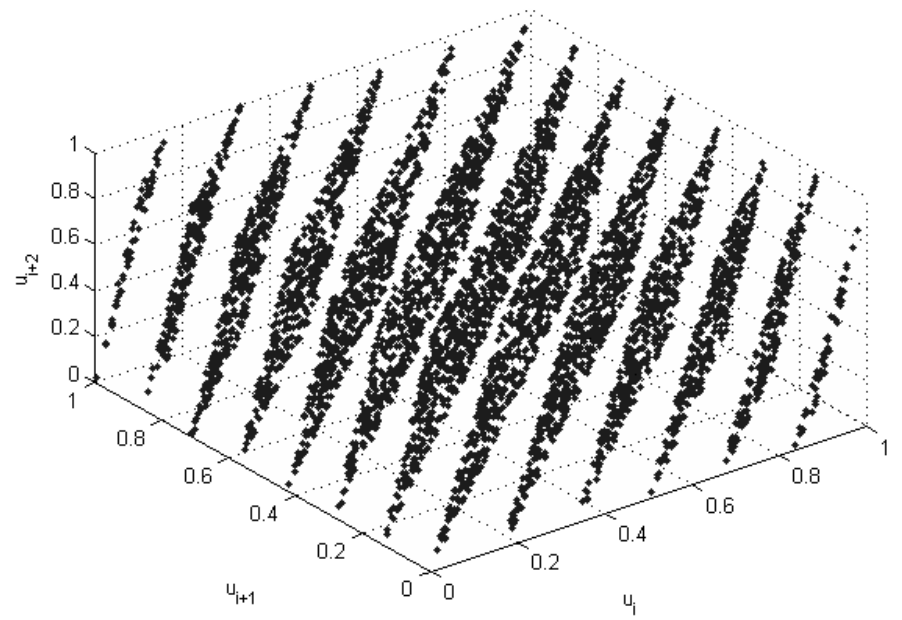

Figura 7. Gerador RANDU: $\operatorname{GCL}\left(\mathbf{2}^{31}-\mathbf{1}, \mathbf{6 5 . 5 3 9 , 0}\right)$. 
No StRAnD é implementado um gerador aparentemente melhor que o RANDU, tal gerador é definido por $\operatorname{GCL}\left(2^{31}-1,41.358,0\right)$, onde a Figura 8 apresenta a partição do hipercubo em duas perspectivas diferentes.
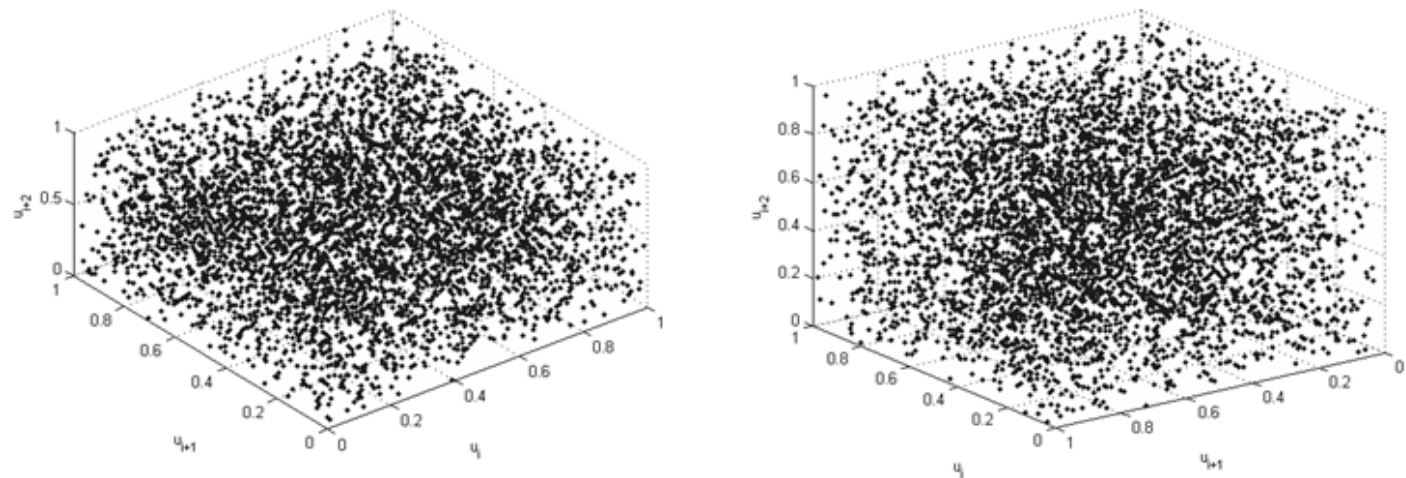

Figura 8. Gerador implementado no StRAnD: $\boldsymbol{G C L}\left(\mathbf{2}^{\mathbf{3 1}}-\mathbf{1}, \mathbf{4 1} . \mathbf{3 5 8}, \mathbf{0}\right)$.

Além do RANDU, existe uma gama de geradores que se encaixam na classe de congruentes lineares.

\subsection{Geração de números aleatórios com distribuição de probabilidade prescrita}

Em confiabilidade de estruturas, principalmente em simulação de Monte Carlo, se está interessado em gerar amostras de variáveis aleatórias. Sabendo-se que uma variável aleatória é definida por sua função de densidade de probabilidade, a partir da função cumulativa de probabilidade $F_{X}(x)$, podem-se obter realizações desta variável aleatória da seguinte forma:

I. Gera-se um número aleatório $u_{i}$ com distribuição uniforme de probabilidade, que está no intervalo $[0,1]$;

II. A partir da inversa da função cumulativa de probabilidade se obtêm o número aleatório com distribuição prescrita, tal que:

$$
x_{i}=F_{X}^{-1}\left(u_{i}\right)
$$

Este procedimento é apresentado na Figura 9. 


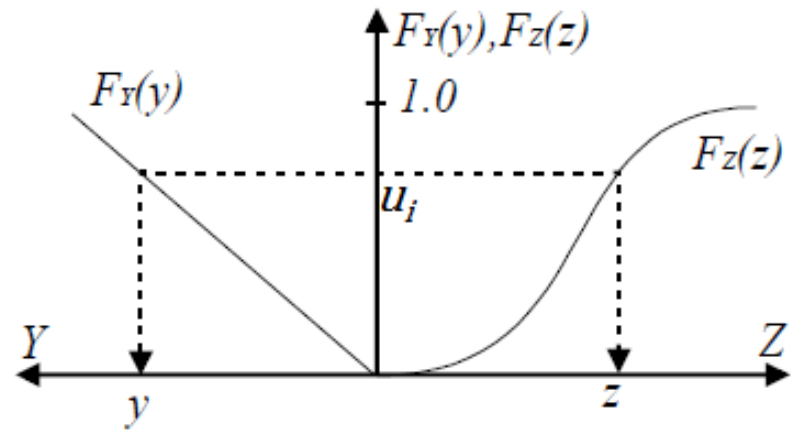

Figura 9. Geração de números aleatórios com distribuição prescrita (BECK, 2012).

Devido a não existência de solução analítica para a função cumulativa de probabilidade, para a distribuição normal e consequentemente log-normal, este procedimento não pode ser aplicado de maneira direta para tais distribuições. Assim recomenda-se a utilização de algoritmos específicos para a resolução deste problema. Pode-se usar a transformação de BoxMuller, esta envolve a adoção de um par de números aleatórios $u_{1}$ e $u_{2}$, independentes e com distribuição de probabilidade uniforme, no intervalo de $[0,1]$, para gerar um par de números aleatórios $y_{1}$ e $y_{2}$, de uma variável aleatória normal padrão (SOONG; GREGORIU, 1993, apud BECK, 2012, p. 140). Os números $y_{1}$ e $y_{2}$ são obtidos da seguinte forma:

$$
\begin{aligned}
& y_{1}=\sqrt{-2 \ln \left(u_{1}\right)} \cos \left(2 \pi u_{2}\right), \\
& y_{2}=\sqrt{-2 \ln \left(u_{1}\right)} \operatorname{sen}\left(2 \pi u_{2}\right) .
\end{aligned}
$$

Para transformar os números aleatórios do espaço normal padrão $\mathbb{Y}$ para o espaço de projeto $\mathbb{X}$, utiliza-se a transformação de Hassofer-Lind, assim têm-se que:

$$
x_{i}=y_{i} \sigma+\mu \text {. }
$$

onde $\mu$ e $\sigma$ é a média e o desvio padrão, respectivamente, da variável X.

Para variáveis com distribuição log-normal, tem-se que:

$$
x_{i}=\exp \left[y_{i} \xi+\lambda\right]
$$

Se as variáveis aleatórias do problema são independentes, então a distribuição conjunta de probabilidades pode ser obtida da seguinte forma:

$$
f_{X}(x)=\prod_{i=1}^{n} f_{X_{i}}\left(x_{i}\right)
$$


Casos as variáveis aleatórias sejam correlacionadas, deve-se adotar um procedimento inverso aquele definido para o FORM, uma vez que no método de confiabilidade de primeira ordem as correlações entre pares de variáveis são eliminadas. No método de Monte Carlo, tais correlações são impostas. Este procedimento pode ser realizado utilizando o modelo de Nataf, para encontrar uma matriz de correlação equivalente $\mathbf{R}_{\mathbf{z}}$. Os passos utilizados para gerar amostras de variáveis aleatórias correlacionadas são (BECK, 2012):

I. Gerar uma amostra normal padrão $\boldsymbol{y}=\left\{y_{1}, y_{2}, \ldots, y_{n}\right\}$;

II. Realizar uma transformação dessas variáveis de $\mathbb{Y} \rightarrow \mathbb{Z}$, através das equações apresentadas em 4.3.1, obtendo assim, um conjunto de variáveis $\boldsymbol{z}=$ $\left\{z_{1}, z_{2}, \ldots, z_{n}\right\}$

III. Define-se o vetor de probabilidades acumuladas $\boldsymbol{u}$ de $\boldsymbol{z}$, tal que $u_{i}=\Phi\left(z_{i}\right)$;

IV. Por fim se obtém as amostras de $\mathbf{X}$ tal como na Eq. (80). 


\section{MÉTODO DE MONTE CARLO}

Em engenharia de estruturas muitas vezes não é viável a realização de certos tipos de experimento, seja pela complexidade de construção ou montagem do equipamento ou modelo, seja pela necessidade de realização de diversas combinações de carregamentos e resistência dos modelos estudados. Partindo deste princípio, as técnicas de simulação surgem como uma alternativa para a observação de estruturas em diferentes configurações de cargas e resistências. Dentre as técnicas de simulação em confiabilidade o método de Monte Carlo surge quando os métodos aproximativos (e.g. FORM e SORM) falham, ou quando se quer testar a resposta obtida por estes métodos.

O método de Monte Carlo foi desenvolvido nos laboratórios de Los Alamos por Metropolis e Ulam (1949) e apresentado no artigo intitulado de The Monte Carlo method. Em confiabilidade de estruturas, o método surge de maneira natural para a simulação de diversas configurações de uma estrutura, de forma que sua aplicação na avaliação da probabilidade de falha seja direta. O método de Monte Carlo é dito Bruto quando este é apresentado em sua forma mais simples. A solução de problemas de confiabilidade através da simulação de Monte Carlo Bruto (BRUTO) é realizada utilizando uma função indicadora $I[\mathbf{x}]$ tal que:

$$
\begin{aligned}
& I[\mathbf{x}]=1 \text { se } \mathbf{x} \in \Omega_{\mathrm{f}}, \\
& I[\mathbf{x}]=0 \text { se } \mathbf{x} \in \Omega_{\mathrm{s}},
\end{aligned}
$$

onde $\Omega_{\mathrm{f}}$ é o domínio de falha e $\Omega_{\mathrm{s}}$ é o domínio de segurança. Podem-se estender os limites de integração sobre todo o domínio $\Omega$ das variáveis aleatórias a fim de se calcular a probabilidade de falha, tal que:

$$
P_{f}=\int_{\Omega} I[\mathbf{x}] f_{\mathbf{X}}(\mathbf{x}) d \mathbf{x} \equiv E[I[\mathbf{x}]]
$$

A Eq. (87) corresponde ao valor esperado da função indicadora, que pode ser estimado a partir de uma amostra de tamanho finito $n$ como:

$$
\bar{P}_{f}=\bar{I}[\mathbf{x}]=\frac{1}{n} \sum_{i=1}^{n} I[\mathbf{x}]=\frac{n_{f}}{n}=E\left[\bar{P}_{f}\right]
$$


onde a $\bar{P}_{f}$ é a probabilidade de falha estimada por uma amostra finita, $n$ é o número de pontos amostrais e $n_{f}$ é o número de pontos amostrais dentro do domínio de falha. Pode-se mostrar que a Eq. (88) é um estimador não tendencioso da probabilidade de falha, ou seja, com $n \rightarrow$ $\infty$, tem-se que $\bar{P}_{f} \rightarrow P_{f}($ BECK, 2012).

Por se tratar de estatística computada a partir de amostra de dimensão finita, o estimador na Eq. (88) está sujeito a um erro de amostragem, que corresponde à variância da probabilidade de falha:

$$
\operatorname{Var}\left[\bar{P}_{f}\right]=\frac{1}{n-1} \sum_{i=1}^{n}\left(I\left[\mathbf{x}_{i}\right]-\bar{P}_{f}\right)^{2}
$$

Observa-se que a variância, ou erro estatístico da amostragem, converge para zero à medida que $n \rightarrow \infty$.

Intervalos de confiança podem ser definidos para a estimativa da probabilidade de falha. Definindo-se um nível de confiança $\alpha$, determina-se o valor de $k$, com $k=\Phi^{-1}\left(\frac{1+\alpha}{2}\right)$, onde $\Phi^{-1}$ é a inversa da função de distribuição acumulada normal padrão. Por exemplo, para um nível de confiança de $95 \% k \cong 1,96$. Então intervalos de confiança são obtidos da seguinte forma:

$$
\bar{P}_{f}-k \sqrt{\operatorname{Var}\left[\bar{P}_{f}\right]} \leq \bar{P}_{f} \leq \bar{P}_{f}+k \sqrt{\operatorname{Var}\left[\bar{P}_{f}\right]}
$$

\subsection{Técnicas de amostragem básica}

As técnicas de amostragem básica são aquelas utilizadas para gerar a amostra necessária na simulação de Monte Carlo. São ditas básicas pelo fato de não necessitar de informações adicionais, como forma da equação de estado limite e pontos de projeto. A seguir serão apresentadas a Amostragem Simples, a Amostragem por Variáveis Antitéticas, a Amostragem Estratificada e a Amostragem por Hipercubo Latino.

\subsubsection{Amostragem simples}

A Amostragem Simples consiste na aplicação direta do procedimento exposto em 5.2, no Monte Carlo Bruto. Gráficos de convergência da probabilidade de falha com o tamanho da amostra podem ser obtidos para avaliar a precisão e a acurácia da simulação. Assim, como apresentado na Figura 10, os gráficos de convergência são compostos pela curva da estimativa 
da probabilidade de falha e pelos intervalos de confiança, tal como definidos na Eq. (90). Assim, quanto maior for o tamanho da amostra, mais estreito vai se tornando o intervalo de confiança. Este tipo de representação gráfica é de particular importância no desenvolvimento deste trabalho, uma vez que a comparação entre as técnicas de amostragem inteligente se baseará neste tipo de resultado. A Figura 10 apresenta um típico gráfico de convergência obtido para o Exemplo 1, que será apresentado em 7.1.

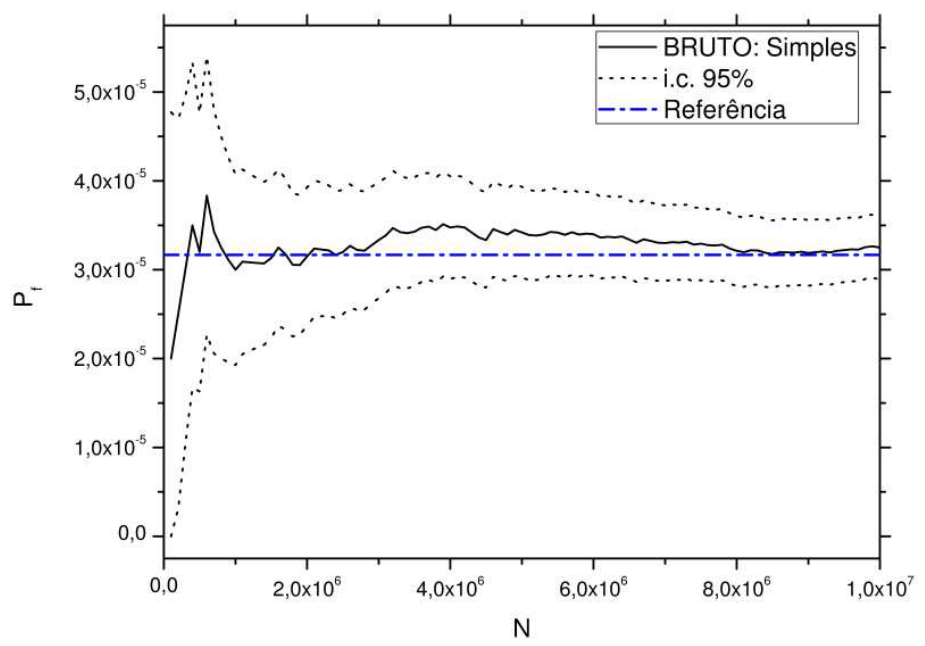

Figura 10. Gráfico de convergência da probabilidade de falha pela simulação do Monte Carlo Bruto, no Exemplo 1.

O coeficiente de variação $\delta$ da probabilidade de falha estimada é dado por:

$$
\delta\left[\bar{P}_{f}\right] \equiv \frac{\sqrt{\operatorname{Var}\left[\bar{P}_{f}\right]}}{E\left[\bar{P}_{f}\right]} \approx \frac{1}{\sqrt{n P_{f}}} .
$$

A partir da Eq. (91), estima-se que para uma probabilidade de falha da ordem de $10^{-p}$ com $\delta\left[\bar{P}_{f}\right] \leq 10 \%$, seja necessária uma amostra de tamanho $10^{p+2}$. Como exemplo, para c. o. v $\left[\bar{P}_{f}\right] \leq 10 \%$ e $P_{f}=10^{-6}$, resulta que $n \geq 10^{6+2}=10^{8}$. Como esta é uma ordem de probabilidade de falha comum em engenharia de estruturas, resulta que o número de simulações necessário para obter uma boa estimativa da probabilidade de falha é elevado (BECK, 2012).

Nos problemas que possuem a equação de estado limite analítica, este número de simulações não é proibitivo. No entanto, a engenharia de estruturas pode utilizar procedimentos de simulação numérica, de elevado custo computacional. Para este tipo de problema, a simulação de Monte Carlo com amostragem simples pode ter custo 
computacional proibitivo, mesmo levando-se em conta os recentes avanços na capacidade de processamento dos computadores.

\subsubsection{Amostragem por variáveis antitéticas}

Na técnica de Amostragem por Variáveis Antitéticas, se realiza a simulação do conjunto de números aleatórios $U=\left\{u_{1}, u_{2}, \ldots, u_{n}\right\}$ de maneira usual, além disso, se realiza a simulação de um conjunto de números, que são complementos dos elementos do conjunto $U$, tal que, $\bar{U}=\left\{1-u_{1}, 1-u_{2}, \ldots, 1-u_{n}\right\}$. A principal proposta deste método é a inserção de correlação negativa entre observações. Dois estimadores não tendenciosos da probabilidade de falha, $P_{f}^{a}$ e $P_{f}^{b}$, podem ser combinados gerando um terceiro estimador não tendencioso $P_{f}^{c}$, tal que:

$$
P_{f}^{c}=\frac{P_{f}^{a}+P_{f}^{b}}{2}
$$

Este estimador possui a seguinte variância:

$$
\operatorname{Var}\left[P_{f}^{c}\right]=\frac{1}{4}\left(\operatorname{Var}\left[P_{f}^{a}\right]+\operatorname{Var}\left[P_{f}^{b}\right]+2 \operatorname{Cov}\left[P_{f}^{a}, P_{f}^{b}\right]\right)
$$

Logo, se $P_{f}^{a}=f\left(u_{i}\right)$ e $P_{f}^{b}=f\left(1-u_{i}\right)$, com $i=1, \ldots, n$, então se está impondo uma correlação negativa, levando $\operatorname{Cov}\left[P_{f}^{a}, P_{f}^{b}\right]$ a ter um valor negativo e assim reduzindo a variância de $P_{f}^{c}$. Esta técnica, quando aplicada sozinha, pode não apresentar melhoras expressivas, porém, quando é utilizada em conjunto com outras técnicas, pode apresentar resultados um pouco mais relevantes (BECK, 2012).

\subsubsection{Amostragem estratificada}

Na Amostragem Simples, os pontos são gerados aleatoriamente em todo o domínio, seguindo a função de densidade conjunta de probabilidade $f_{\mathbf{X}}(\mathbf{x})$. Esta amostragem aleatória poderia gerar concentração de pontos em algumas regiões do domínio, e deixar outras regiões com poucos pontos. A amostragem estratificada busca evitar esta falta de homogeneidade na cobertura do espaço amostral. Isso é realizado dividindo o domínio em faixas e realizando amostragens em cada faixa do domínio (McKAY; BECKMAN; CONOVER, 1979). A Figura 11 ilustra a geração de pontos na amostragem estratificada para duas variáveis aleatórias. Porém, esta técnica não se mostra tão eficiente quanto outras técnicas de amostragem 
inteligente, o que a tornaria um método obsoleto e sem muita utilização dentro do StRAnD, por isso, ela não é objeto de estudo deste trabalho.

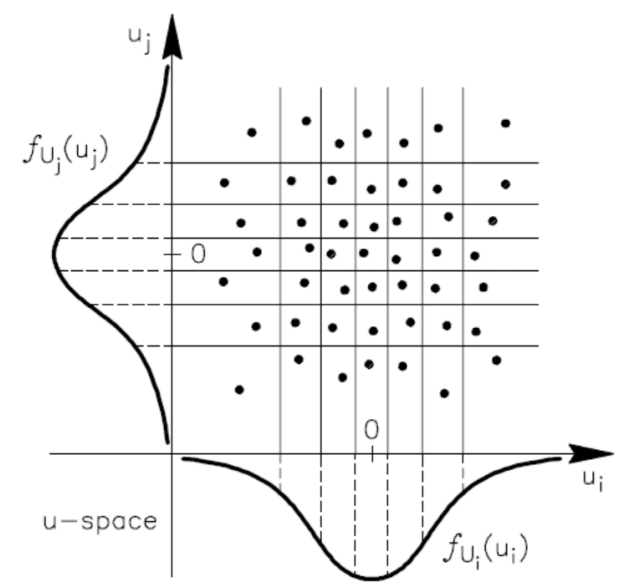

Figura 11. Amostragem estratificada (HURTADO; BARBAT, 1998).

\subsubsection{Amostragem por hipercubo latino}

A técnica de amostragem por hipercubo latino foi apresentada primeiramente por McKay, Beckman e Conover (1979), onde foi realizado um estudo comparativo com a amostragem aleatória e amostragem estratificada.

De maneira semelhante à amostragem estratificada, a amostragem por hipercubo latino divide o domínio de cada variável aleatória em faixas (McKAY; BECKMAN; CONOVER, 1979) e (OLSSON; SANDEBERG; DAHLBLOM, 2003). Portanto, cada faixa é amostrada uma única vez, resultando numa distribuição esparsa dos pontos (Figura 12). Isto garante uma cobertura homogênea do domínio das variáveis aleatórias.

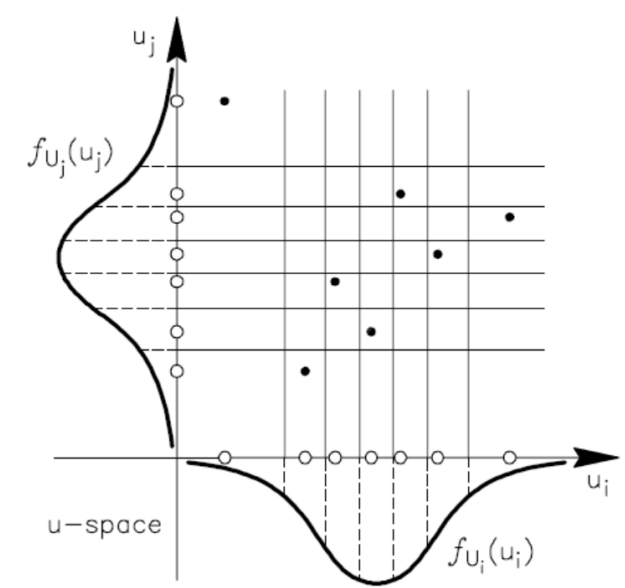

Figura 12. Amostragem por hipercubo latino (HURTADO; BARBAT, 1998). 
Seja $n_{v a}$ o número de variáveis aleatórias do problema e $n$ o número de pontos da amostra. $\mathrm{O}$ espaço de amostragem torna-se $n_{v a}$-dimensional. Uma matriz $\mathbf{P}$, de dimensões $\left(n \times n_{v a}\right)$, é gerada, onde cada uma das $n_{v a}$ colunas é uma permutação aleatória de $1, \ldots, n$. Outra matriz $\mathbf{R}$ é gerada (dimensões $n \times n_{v a}$ ), cujos componentes são números aleatoriamente distribuídos entre $(0,1)$. A partir destas matrizes, obtém-se a matriz S (OLSSON; SANDEBERG; DAHLBLOM, 2003):

$$
\mathbf{S}=\frac{1}{n}(\mathbf{P}-\mathbf{R}) .
$$

Como exemplo, na Figura 13 considere as dimensões $\left(n \times n_{v a}\right)=(5 \times 2)$, assim:

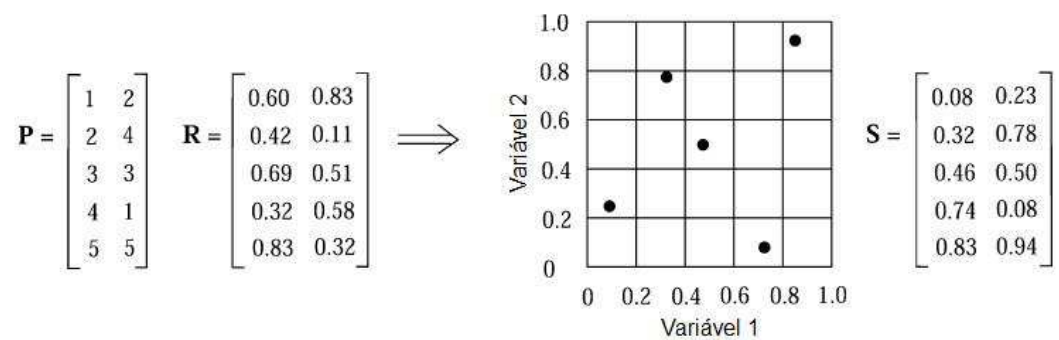

Figura 13. Hipercubo Latino para duas variáveis e cinco realizações (OLSSON; SANDEBERG;

DAHLBLOM, 2003).

Assim as amostras são geradas a partir de $\mathbf{S}$, tal que:

$$
x_{i j}=F_{x j}^{-1}\left(s_{i j}\right)
$$

onde $F_{x j}^{-1}$ é a inversa da função de distribuição acumulada de probabilidade da variável $X_{j}$.

A geração das matrizes $\mathbf{S}, \mathbf{P}$ e $\mathbf{R}$, pode ser proibitiva quando se deseja gerar uma amostra de tamanho $n$ muito grande, uma vez que isto pode levar a um consumo excessivo de memória do computador. No StRAnD esta técnica está programada tanto da maneira apresentada anteriormente, quanto de maneira a reduzir o consumo de memória. Os passos adotados para reduzir o consumo de memória são os seguintes:

1. Inicia-se o Loop para a variável aleatória j;

2. Gera-se um vetor, que é a primeira coluna da matriz $\mathbf{P}$;

3. Inicia-se o loop para o número de simulações $i$;

4. Gera-se um número aleatório entre 0 e 1 , sem ser necessário gerar uma matriz $\mathbf{R}$ ou qualquer tipo de Array; 
5. Calcula-se um escalar $s$, sem a necessidade de se criar uma matriz $\mathbf{S}$ e aplica-o na Eq. (95) para calcular os elementos $x_{i j}$;

6. Retorna ao passo 4 até $i=n$;

7. Retorna ao passo 2 até $j=n_{v a}$

Desta forma, o histograma de frequência das realizações de cada variável aleatória adquire uma forma mais suave. Tal comportamento pode ser observado na Figura 14, onde se destacam os histogramas para a Amostragem Simples, para a Amostragem por Variáveis Antitéticas e para a Amostragem por Hipercubo Latino para uma variável aleatória normal com média 1,0 e desvio padrão de 0,15 .

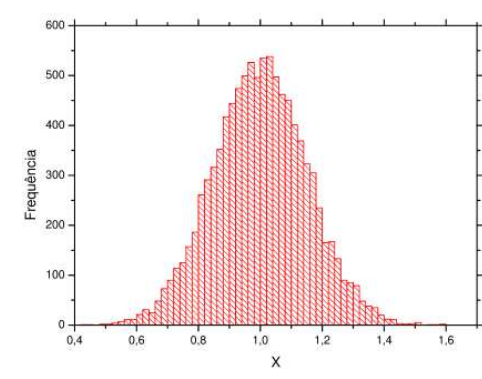

a)

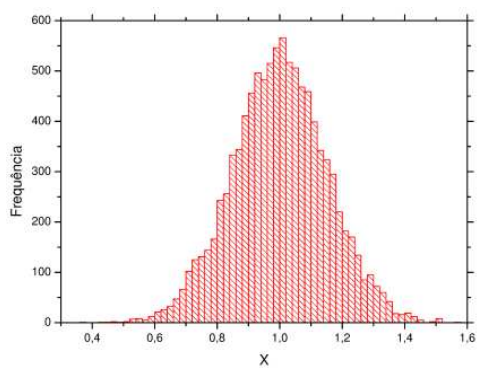

b)

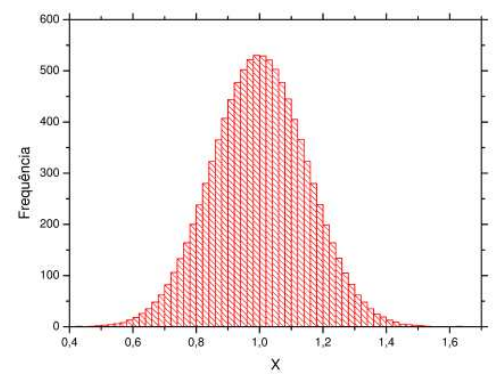

c)

Figura 14. Histograma de frequência na a) Amostragem Simples, b) Amostragem por Variáveis Antitéticas e c) Amostragem por Hipercubo Latino.

\subsection{Técnicas de Amostragem Inteligentes}

A seguir são apresentadas algumas técnicas que são propostas para estimar a probabilidade de falha de problemas onde o Monte Carlo Bruto apresenta dificuldades, tais como em problemas com probabilidade de falha pequenas, na avaliação em conjunto com um

problema numérico (e.g. Elementos Finitos) ou na avaliação de sistemas. É dado destaque a Amostragem por Importância utilizando pontos de projeto (AI), a Amostragem Assintótica (AA), a Amostragem Melhorada (AM) e a Simulação de Subconjuntos (SS).

Vale destacar que as técnicas de Amostragem por Hipercubo Latino e de Amostragem por Variáveis Antitéticas, são técnicas de amostragem básica e que também são consideradas como técnicas de amostragem inteligente, porém, estas já foram apresentadas anteriormente. 


\subsubsection{Amostragem por Importância utilizando pontos de projeto}

A técnica de Amostragem por Importância usando pontos de projeto consiste em transladar os pontos de amostragem para a região do domínio de falha. Para deslocar os pontos de amostragem, os mesmos são gerados a partir de uma função de amostragem $h_{\mathbf{X}}(\mathbf{x})$, convenientemente escolhida. A forma de escolha da função de amostragem dá origem às diferentes técnicas de amostragem por importância. Multiplicando-se numerador e denominador da Eq. (87) por $h_{\mathbf{X}}(\mathbf{x})$, se obtém:

$$
P_{f}=\int_{\Omega} I(\mathbf{x}) \frac{f_{\mathbf{X}}(\mathbf{x})}{h_{\mathbf{X}}(\mathbf{x})} h_{\mathbf{X}}(\mathbf{x}) d x .
$$

Esta é uma expressão para o valor esperado da função $I(\mathbf{x}) \frac{f_{\mathbf{X}}(\mathbf{x})}{h_{\mathbf{X}}(\mathbf{x})}$. Este valor esperado pode ser estimado a partir de uma amostra de tamanho finito, tal que:

$$
\bar{P}_{f}=\bar{I}(\mathbf{x})=\frac{1}{n} \sum_{i=1}^{n} I\left(\mathbf{x}_{i}\right) \frac{f_{\mathbf{X}}\left(\mathbf{x}_{i}\right)}{h_{\mathbf{X}}\left(\mathbf{x}_{i}\right)} .
$$

Ao deslocar a amostra para o domínio de falha, a função indicadora terá mais valores 1 do que na amostra original. No entanto, observa-se que cada valor 1 da função indicadora está associado a um peso definido por $w_{i}=\frac{f_{\mathbf{X}}\left(\mathbf{x}_{i}\right)}{h_{\mathbf{X}}\left(\mathbf{x}_{i}\right)} \ll 1(\mathrm{BECK}, 2012)$.

A qualidade do resultado obtido pela Amostragem por Importância depende muito da função de amostragem que se adota. Uma função de amostragem mal escolhida pode até piorar o resultado. A literatura apresenta diversas maneiras de se propor a função de amostragem, porém, a técnica abordada neste trabalho é a amostragem por importância utilizando pontos de projeto. Esta técnica apresenta diversas vantagens, como a rápida convergência da resposta, onde como consequência é possível se obter uma baixa variância da probabilidade de falha, em comparação com o Monte Carlo Bruto. A Figura 15 apresenta um típico gráfico de convergência obtido para o Exemplo 1, que será apresentado em 7.1, onde se compara a convergência da probabilidade de falha para o Monte Carlo Bruto (BRUTO) e para a Amostragem por Importância (AI). A Figura 16, apresenta o gráfico apenas da Amostragem por Importância. 


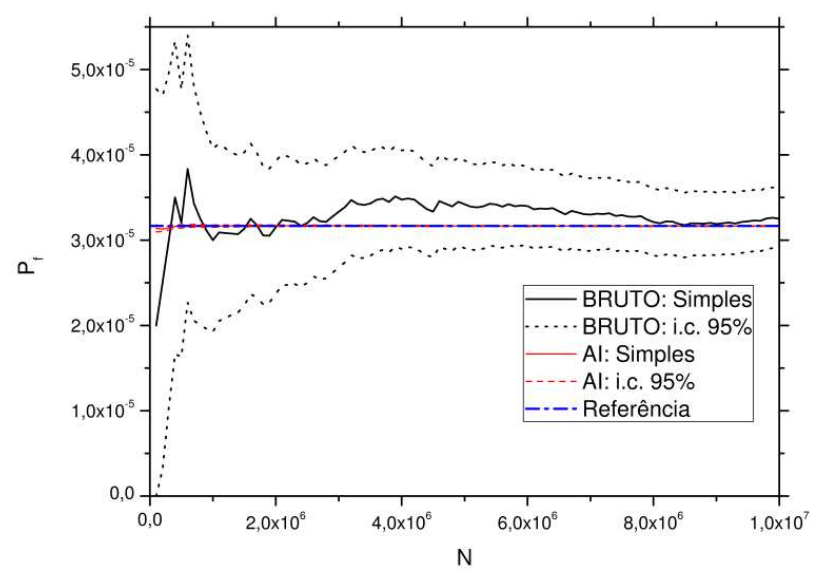

Figura 15. Comparativo da convergência do Monte Carlo Bruto com a Amostragem por Importância, no Exemplo 1.

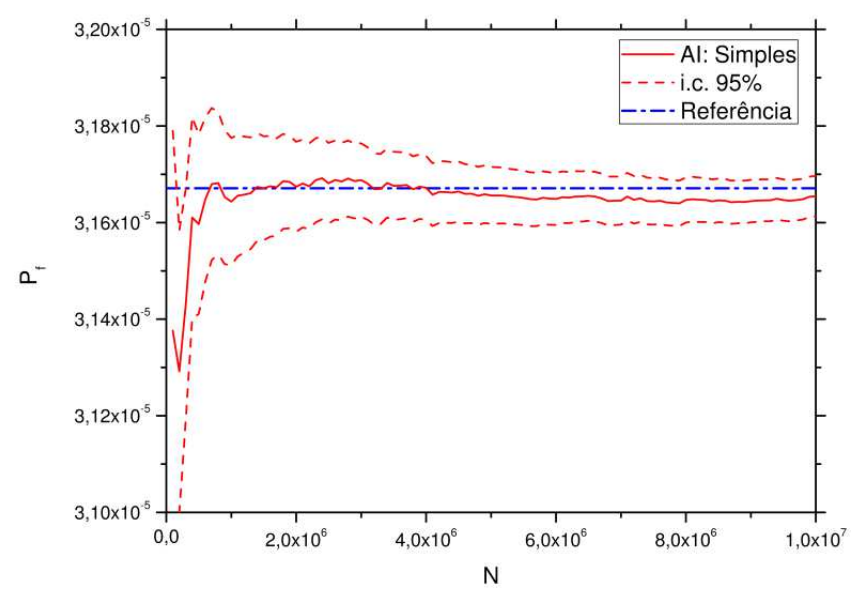

Figura 16. Convergência do Monte Carlo com Amostragem por Importância, no Exemplo 1.

\subsubsection{Amostragem por Importância utilizando Hipercubo Latino}

Enquanto que a aplicação da Amostragem Simples e da Amostragem por Variáveis Antitéticas é feita de maneira direta na Amostragem por Importância, neste trabalho se optou pela modificação apresentada por Olsson, Sandberg e Dahlblom (2003). Segundo Olsson, Sandberg e Dahlblom (2003), na amostragem por importância com hipercubo latino padrão, existe a possibilidade de que a maioria das realizações das variáveis aleatórias do problema aconteça apenas em um lado da hipersuperfície de falha. Isto pode ser evitado aplicando uma transformação ao hipercubo latino. A transformação é aplicada ao hipercubo latino gerado, de forma que uma de suas direções coincida com a direção definida pelo vetor unitário do ponto de projeto $\widehat{\boldsymbol{y}}$, que é o vetor definido pelas coordenadas da origem do espaço de probabilidade 
e pelas coordenadas do ponto de projeto. Na Figura 17 podemos observar o vetor do ponto de projeto $\boldsymbol{y}^{*}$ no espaço bidimensional.

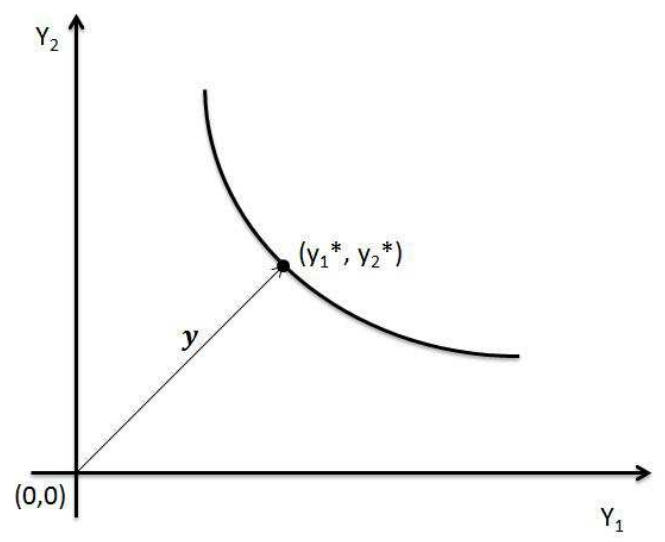

Figura 17. Vetor do ponto de projeto.

O vetor do ponto de projeto de dimensão $n$ é definido por:

$$
\boldsymbol{y}^{*}=\left\langle y_{1}^{*}, y_{2}^{*}, \ldots, y_{n}^{*}\right\rangle=\left\langle\left(y_{1}^{*}, y_{2}^{*}, \ldots, y_{n}^{*}\right)-(0,0, \ldots, 0)\right\rangle \text {. }
$$

O vetor unitário do ponto de projeto é dado pela razão entre o vetor do ponto de projeto $\boldsymbol{y}^{*}$ e sua norma $\left|\boldsymbol{y}^{*}\right|$, assim temos que:

$$
\widehat{\boldsymbol{y}}^{*}=\frac{\boldsymbol{y}^{*}}{\left|\boldsymbol{y}^{*}\right|}
$$

Dessa forma, uma particular direção do espaço que gera o hipercubo latino é transformada de forma a coincidir com $\widehat{\boldsymbol{y}}^{*}$, as direções remanescentes são definidas como direções ortogonais a $\widehat{\boldsymbol{y}}^{*}$, assim pode-se definir uma matriz de transformação $\boldsymbol{A}$. As linhas de $\boldsymbol{A}$ são compostas pelas componentes dos vetores unitários do espaço transformado lidos no espaço original. Uma propriedade de $\boldsymbol{A}$ é que devido à ortogonalidade dos vetores da base do espaço vetorial em questão, $\boldsymbol{A}$ é uma matriz ortogonal de forma que:

$$
\boldsymbol{A}^{T} \boldsymbol{A}=\boldsymbol{A}^{-1} \boldsymbol{A}=\boldsymbol{I}
$$

A matriz de transformação $\boldsymbol{A}$ é obtida via processo de ortogonalização de Gram-Schmidt. Dessa forma, inicialmente é gerada uma amostra com distribuição normal padrão não correlacionada $\boldsymbol{u}_{j}=\left(u_{1}, u_{2}, \ldots, u_{n_{r v}}\right)$ com $j=1,2, \ldots, n$, na origem. Em seguida aplica-se a matriz de transformação na amostra, de forma que: 


$$
\boldsymbol{u}_{j}^{*}=A \boldsymbol{u}_{j}
$$

Então as amostras transformadas são deslocadas para o ponto de projeto de forma que:

$$
\widetilde{\boldsymbol{u}}_{j}^{*}=\boldsymbol{u}_{j}^{*}+\boldsymbol{y}^{*}
$$

A Figura 18 apresenta a transformação do hipercubo latino no espaço bidimensional.
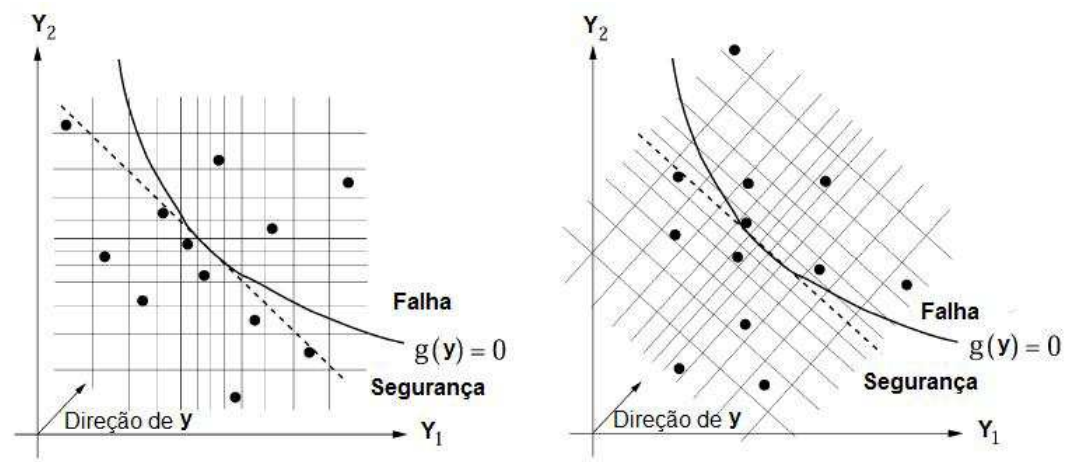

Figura 18. Hipercubo Latino original e transformado, adaptado de (OLSSON; SANDEBERG e DAHLBLOM, 2003).

A seguir serão apresentadas como se dá a aplicação da Amostragem por Importância em sistemas.

\subsubsection{Múltiplos modos de falha}

Havendo um único modo de falha, ou apenas uma equação de estado limite, a construção da função de amostragem utilizando o ponto de projeto é simples: a função $h_{\mathbf{X}}(\mathbf{x})$ é construída tomando-se a função $f_{\mathbf{X}}(\mathbf{x})$ e substituindo a média pelas coordenadas do ponto de projeto $\mathbf{x}^{*}$. Havendo mais de uma equação de estado limite, é necessário levar em consideração a forma de associação destas (se em série ou em paralelo) e levar em conta a importância relativa de cada estado limite. Para múltiplos modos de falha, a função de amostragem é construída como uma soma ponderada (BECK, 2012), tal que:

$$
h_{\mathbf{X}}(\mathbf{x})=\sum_{i=1}^{N_{e l}} p_{i} h_{i \mathbf{X}}(\mathbf{x})
$$

onde $N_{e l}$ é o número de equações de estado limite, $p_{i}$ é o peso associado a $i$-ésima equação de estado limite e $h_{i \mathbf{X}}(\mathbf{x})$ é uma função de amostragem centrada no $i$-ésimo ponto de projeto. Os pesos $p_{i}$ são determinados a partir da importância relativa de cada modo de falha. Para sistemas em série, pode-se considerar: 


$$
p_{i}=\frac{\Phi\left(-\beta_{i}\right)}{\sum_{i=1}^{n l s} \Phi\left(-\beta_{i}\right)}
$$

Assim, o tamanho total da amostra é tomado como uma soma ponderada da amostra relativa a cada estado limite, tal que:

$$
n_{i}=p_{i} n
$$

A Figura 19 ilustra a construção de uma função de amostragem centrada sobre dois pontos de projeto, para um problema com duas equações de estado limite.

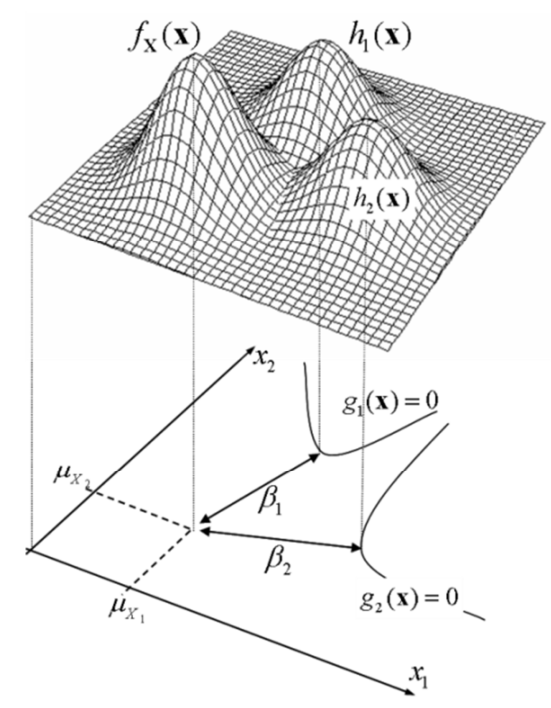

Figura 19. Amostragem por importância utilizando pontos de projeto (BECK, 2012).

Esta técnica apresenta o inconveniente de necessitar do conhecimento prévio do ponto, ou dos pontos, de projeto, uma vez que a função de amostragem precisa ser transladada até ele, ou eles. Isso faz com que problemas para os quais não é possível definir os pontos de projeto, não apresentem resposta por este método, sendo necessário aplicar técnicas que não façam uso desta informação adicional.

\subsubsection{Amostragem assintótica}

Bucher (2009) introduziu uma nova técnica que utiliza o método de Monte Carlo e cuja finalidade é estimar baixas probabilidades de falha. Tal técnica é a Amostragem Assintótica. Esta técnica faz uso das propriedades assintóticas da probabilidade de falha à medida que os desvios-padrão das variáveis aleatórias tendem a zero. A técnica dispensa o conhecimento do ponto de projeto. 
Inicialmente, introduz-se um fator $f$ que é inversamente proporcional aos desviospadrão $\sigma$ das variáveis aleatórias do problema:

$$
f \propto \frac{1}{\sigma}
$$

Sabe-se que o índice de confiabilidade $\beta$ torna-se proporcional ao fator $f$. Portanto, pode-se aproximar a relação funcional entre $\beta$ e $f$ como (BUCHER, 2009):

$$
\beta=A \cdot f+\frac{B}{f}
$$

onde $A$ e $B$ são constantes a determinar. Desta forma se garante o comportamento assintótico linear à medida que $f \rightarrow \infty$. Outras funções poderiam ser escolhidas de acordo com o comportamento das duplas $(f, \beta)$, que são os pontos de suporte (BUCHER, 2009). Estas constantes podem ser determinadas usando técnicas convencionais de ajuste (e.g. mínimos quadrados), a partir da avaliação de duplas $(f, \beta)$. Para o processo de ajuste a Eq. (107) é reescrita em termos da razão do índice de confiabilidade $\beta$ com o fator $f$ (BUCHER, 2009):

$$
\frac{\beta}{f}=A+\frac{B}{f^{2}}
$$

Esta reformulação automaticamente atribui pesos iguais a todos os pontos de suporte (BUCHER, 2009). As duplas guardadas são $(f, \beta / f)$, o que leva a obtenção de um gráfico tal como a ilustração mostrada na Figura 20.

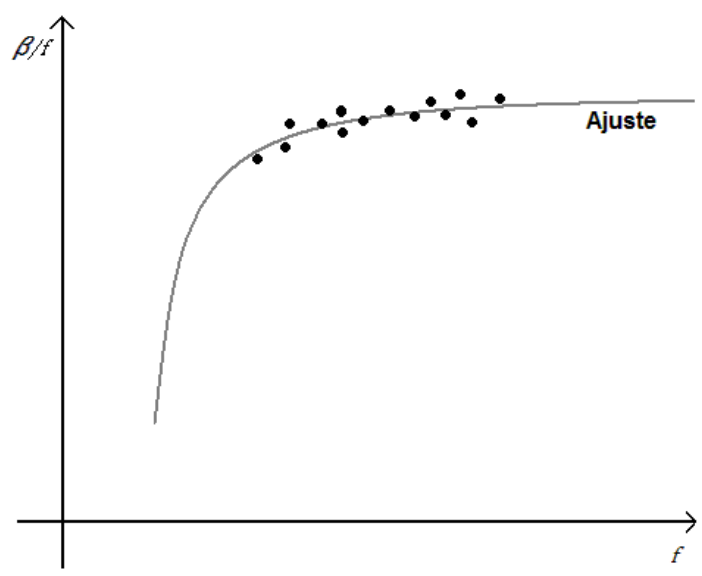

Figura 20. Ilustração do ajuste não linear realizado nas duplas $\left(\boldsymbol{f}, \frac{\boldsymbol{\beta}}{\boldsymbol{f}}\right)$.

A Eq. (108) pode ser substituída por uma mais geral, tal que: 


$$
\frac{\beta}{f}=A+B \cdot f^{C}
$$

onde $C$ é um valor que é ajustado juntamente com $A$ e $B$.

Para problemas multidimensionais, pode-se escrever:

$$
\sigma_{i}^{\text {sim }}=\frac{\sigma_{i}}{f}
$$

onde $\sigma_{i}$ é o desvio-padrão da $i$-ésima variável aleatória e $\sigma_{i}^{\text {sim }}$ é o desvio padrão utilizado na simulação, desta forma, $f=1$ representa o problema original. Os intervalos de confiança da probabilidade de falha são obtidos através das bandas de confiança (Intervalos de confiança da resposta média), que são obtidos em toda curva ajustada. O procedimento de obtenção de tais intervalos é apresentado em Montegomery e Runger (2003), onde um intervalo de $100 \times(1-\alpha) \%$ sobre a resposta média, no valor $x=x_{0}$ é obtido, tal que $E\left[Y \mid x_{0}\right]=\mu_{Y \mid x_{0}}$ é o valor médio exato da curva e $\hat{\mu}_{Y \mid x_{0}}$ é o valor estimado da função ajustada no ponto $x_{0}$. Assim, o intervalo de confiança da resposta média é dado por:

$$
\begin{aligned}
\hat{\mu}_{Y \mid x_{0}}-t_{\frac{\alpha}{2}, n-2} \sqrt{Q M E\left(\frac{1}{n}+\frac{\left(x_{0}-\bar{x}\right)^{2}}{\sum_{i=1}^{n}\left(x_{i}-\bar{x}\right)^{2}}\right)} \leq \mu_{Y \mid x_{0}} \\
\leq \hat{\mu}_{Y \mid x_{0}}+t_{\frac{\alpha}{2}, n-2} \sqrt{Q M E\left(\frac{1}{n}+\frac{\left(x_{0}-\bar{x}\right)^{2}}{\sum_{i=1}^{n}\left(x_{i}-\bar{x}\right)^{2}}\right)}
\end{aligned}
$$

onde

$$
Q M E=\frac{\sum_{i=1}^{n}\left(Y_{i}-\hat{Y}_{i}\right)^{2}}{n-p}
$$

tal que, $Y_{i}$ é o valor do ponto de suporte, $\widehat{Y}_{i}$ é o valor da função ajustada para um valor $x$ do ponto de suporte, $n$ é o número de pontos de suporte e $p$ é o número de parâmetros.

Em resumo, para resolver um problema de simulação utilizando amostragem assintótica, escolhe-se um intervalo de validade para $f$ e um valor $n_{0}$ que corresponde ao número requerido de pontos no domínio de falha para obter boa precisão no $\beta$ calculado. $\mathrm{O}$ resultado final é obtido para a Eq. (108) ou Eq. (109) com $f=1$. Na Figura 21 é possível se 
ter uma boa visão da aplicação do método quando se parametriza o desvio-padrão de cada variável aleatória.
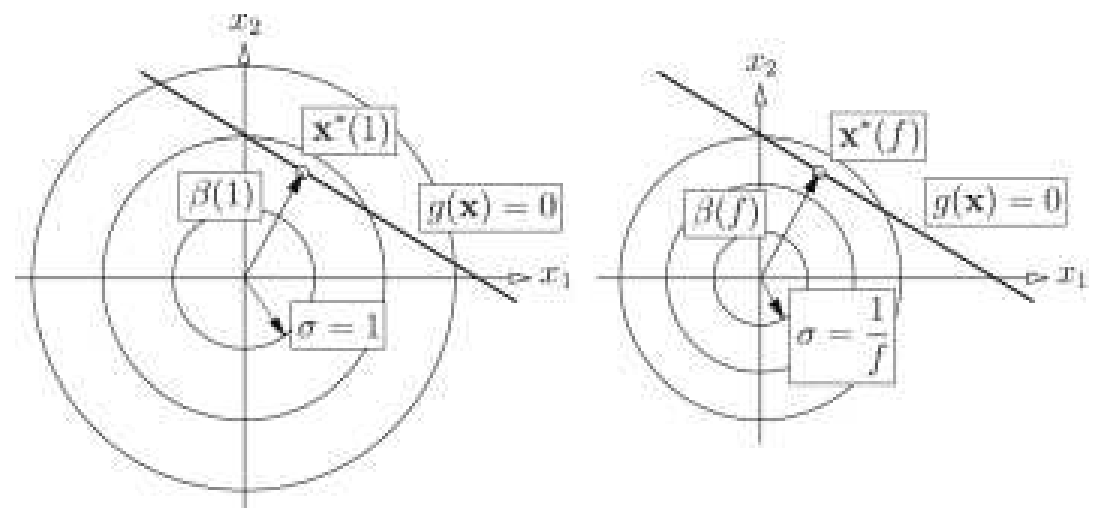

Figura 21. Parametrização dos desvios-padrão das variáveis aleatórias (SICHANI; NIELSEN; BUCHER, 2011b).

Uma importante questão referente ao uso desta técnica é a escolha dos pontos de suporte, de forma que seja possível obter resultados satisfatórios. Sichani, Nielsen e Bucher (2011a,b) mostraram que é necessário estabelecer com critérios o intervalo de $f$ definido por $\left[f_{\text {mín }}, f_{\text {máx }}\right]$, de forma que seja possível obter bons resultados. Também foi mostrado que existe um intervalo $\left[f_{\text {mín }}, f_{\text {máx }}\right]$ ótimo, de forma que o erro em relação ao valor médio de diversas realizações do método fosse o menor possível, porém, a largura do intervalo do parâmetro $f$ depende do tipo do problema que se esteja analisando, i.e. a forma da equação de estado limite, o nível da barreira, etc. Vale destacar que o algoritmo desenvolvido por Sichani, Nielsen e Bucher (2011a), para definir tal intervalo, se justifica em face ao seu alto custo computacional quando se esta trabalhando com problemas de dinâmica estocástica. Como este trabalho analisará problemas de confiabilidade independentes do tempo, um algoritmo desse tipo pode apresentar um alto custo computacional.

Apesar dos benefícios apresentados pela técnica, deve-se prestar atenção a possibilidade do mesmo apresentar algumas inconsistências, pois, uma vez que o desvio padrão das variáveis aleatórias é modificado, existe a possibilidade de simulação de valores não consistentes com as propriedades físicas das variáveis e muitas vezes isso leva a erros na estimativa da probabilidade de falha de cada ponto de suporte, aumentando a incerteza da resposta. Como exemplo, tomando um problema com duas variáveis aleatórias uniformes em um problema do tipo capacidade demanda (C-D), tal que a variável $\mathrm{C} \sim \mathrm{U}(1,1 ; 10,1)$ e D U(10;30). Sendo assim os desvios padrão delas são 6,75 e 8,3333, respectivamente. Assim, dividindo os desvios-padrão por 0,6 e 0,375, os limites da distribuição uniforme passam a ser: $[\text { mín,máx }]_{0,6}=[3,3333 ; 36,6667]$ e $\quad[\text { mín,máx }]_{0,375}=[-6,6667 ; 46,6667]$ para a 
variável Capacidade e $[\text { mín, máx }]_{0,6}=[-1,9 ; 13,1]$ e $[\text { mín, máx }]_{0,375}=[-6,4 ; 17,6]$, para a variável Demanda. Tal comportamento é apresentado na Figura 22.

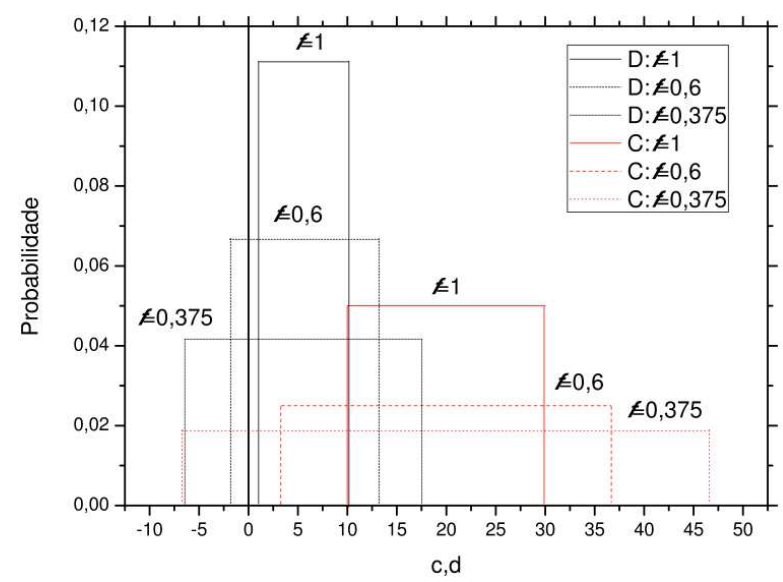

Figura 22. Distribuições de probabilidade parametrizadas das variáveis: Capacidade (C) e Demanda (D).

De acordo com a Figura 22, como a distribuição é uniforme, então para cada distribuição parametrizada por $f$, é possível obter qualquer resultado, com a mesma probabilidade, entre os valores máximos e mínimos que foram anteriormente apresentados. Dessa forma, deverá ser questionado se as variáveis podem apresentar valores negativos, o que muitas vezes poderá representar um dado fisicamente inconsistente. Dessa forma o StRAnD está provido de um algoritmo que pode ser ativado pelo usuário, através do arquivo de entrada, para caso seja necessário avaliar tais condições. O Algoritmo identifica para cada valor de $f$, se os valores máximos e mínimos da distribuição de probabilidade parametrizada possuem o mesmo sinal da distribuição original. Dessa forma o programa deverá identificar um intervalo do parâmetro $f$, que seja adequado a essa condição. Além disso, esta técnica poderá apresentar problemas quando as variáveis aleatórias não possuem caudas com decrescimento suave, como no caso da distribuição uniforme. Este último problema será discutido em 7.2.

Por estar em desenvolvimento, esta técnica ainda é objeto de vários estudos, tais como os apresentados por Tang, Lu e Pan (2014), que discutem três questões. A primeira se refere ao custo computacional da Amostragem Assintótica, que pode ser proibitivo devido à necessidade de avaliações independentes de $\beta\left(f_{i}\right)$ para a obtenção dos pontos de suporte. A segunda diz respeito às possíveis discrepâncias na exatidão do resultado proveniente do uso da Eq. (107). A terceira questão abordada pelos autores refere-se ao fato de que a Amostragem Assintótica pode não ser capaz de gerar intervalos de confiança. Já Sichani, Nielsen e Bucher (2014), explicam que a técnica falha para problemas de dimensão elevada. 


\subsubsection{Amostragem melhorada}

Uma técnica em simulação de Monte Carlo foi proposta por Naess, Leira e Batsevych (2009) com o objetivo de estimar a confiabilidade de sistemas, reduzindo o custo computacional e mantendo a funcionalidade do método de Monte Carlo. Segundo Naess, Leira e Batsevych (2009), esta técnica explora a regularidade das probabilidades nas caudas das distribuições de probabilidade, de forma que um procedimento aproximativo possa ser aplicado na estimativa de probabilidades de falha pequenas. $\mathrm{O}$ procedimento adotado na Amostragem Melhorada é apresentado a seguir.

Uma função margem de segurança $M=g\left(x_{1}, x_{2}, \ldots, x_{n}\right)$, que é expressa em termos de $n$ variáveis aleatórias, é a base de um conjunto de equações parametrizadas de $M$. Assim, o conjuntos de funções $M$ é definido pelo parâmetro $\lambda$, com $0 \leq \lambda \leq 1$. Dessa forma:

$$
M(\lambda)=M-(1-\lambda) \cdot \mu_{M}
$$

onde $\mu_{M}$ é a média da função margem de segurança. Então, assume-se que o comportamento das duplas $\left[\lambda, p_{f}(\lambda)\right]$ é governado pela equação:

$$
p_{f}(\lambda) \approx q(\lambda) \exp \left\{-a(\lambda-b)^{c}\right\} \operatorname{com} \lambda \rightarrow 1 \text {. }
$$

A função $q(\lambda)$ varia pouco em comparação à $\exp \left\{-a(\lambda-b)^{c}\right\}$. Então, para um intervalo $\lambda_{0} \leq \lambda \leq 1$, pode-se considerar a função $q(\lambda)$ constante, tal que:

$$
p_{f}(\lambda) \approx q \cdot \exp \left\{-a(\lambda-b)^{c}\right\} \operatorname{com} \lambda \rightarrow 1
$$

Conhecendo as duplas $\left[\lambda, p_{f}(\lambda)\right]$, pode-se ajustar os parâmetros da Eq. (115), por meio do algoritmo de ajuste não linear contido no StRAnD. Assim, para $\lambda=1$, pode-se estimar o valor da probabilidade de falha, fazendo $p_{f}=p_{f}(1)$. O intervalo de confiança de $p_{f}$ é o intervalo de confiança obtido pela banda de confiança do ajuste não linear (Intervalo de confiança na resposta média), que foi apresentado em 6.2.2. Tal abordagem diferencia-se daquela adotada por Naess, Leira e Batsevych (2009), uma vez que o autor utiliza bandas de confiança empíricas na estimativa dos intervalos de confiança.

\subsubsection{Múltiplos modos de falha}

Segundo Naess, Leira e Batsevych (2009), sendo as funções margem de segurança, para cada componente de um sistema, definidas por $M_{j}=g_{j}\left(x_{1}, x_{2}, \ldots, x_{n}\right)$, com $j=1, \ldots, m$, 
onde $m$ é o número de componentes do sistema e $n$ o número de variáveis aleatórias, então se pode parametrizar este conjunto de equações da seguinte forma:

$$
M_{j}(\lambda)=M_{j}-(1-\lambda) \cdot \mu_{M_{j}}
$$

Assim, a definição para um sistema em série é dada por:

$$
p_{f}=P\left(\bigcup_{j=1}^{m}\left\{M_{j}(\lambda) \leq 0\right\}\right) .
$$

Para um sistema em paralelo é dada por:

$$
p_{f}=P\left(\bigcap_{j=1}^{m}\left\{M_{j}(\lambda) \leq 0\right\}\right) \text {. }
$$

Para um sistema em série formado por subsistemas em paralelo, tem-se:

$$
p_{f}=P\left(\bigcup_{j=1}^{l} \bigcap_{i \in C_{j}}\left\{M_{i}(\lambda) \leq 0\right\}\right) \text {, }
$$

com $C_{j}$, sendo um subconjunto de $1, \ldots, m$, para $j=1, \ldots, l$.

Dessa forma, utiliza-se a Eq. (115) para o ajuste não linear das duplas $\left[\lambda, p_{f}(\lambda)\right]$ na avaliação de sistemas.

\subsubsection{Simulação de subconjuntos}

A técnica de Simulação de Subconjuntos foi proposta por Au e Beck (2001) com a finalidade de estimar probabilidades de falha pequenas em análise de confiabilidade de sistemas. A ideia da técnica é definir o evento falha, que possui baixa probabilidade de ocorrência, como uma sequência de eventos com probabilidades de falha maior, porém condicionais. Assim, não é necessário ter uma amostra grande na avaliação de pequenas probabilidades de falha.

O método de Monte Carlo Bruto não é considerado uma boa alternativa para estimar as probabilidades condicionais. Assim o método de Monte Carlo com Cadeias de Markov (Markov Chain Monte Carlo - MCMC), que faz uso do algoritmo de Metropolis-Hastings, é uma alternativa natural para tal aplicação. 
A ideia fundamental da Simulação de Subconjuntos (Subset Simulation) é substituir o cálculo de uma probabilidade de falha muito pequena, de elevado custo computacional, por uma sequência ou produto de probabilidades de falha condicionais. Isso é feito através de escolha apropriada dos limites de falha intermediários, assim, a probabilidade de falha dos eventos condicionais pode ser estimada com um número reduzido de simulações. Para estimar as probabilidades dos eventos condicionais, utilizam-se as cadeias de Markov e o algoritmo de Metropolis-Hastings Modificado. A definição matemática da técnica é dada a seguir.

Seja $E$ o evento falha, cuja probabilidade se deseja estimar:

$$
P[E]=P\left[\mathbf{X} \in \Omega_{\mathrm{f}}\right]=\int_{\Omega_{\mathrm{f}}} f_{\mathbf{X}}(\mathbf{x}) d \mathbf{x}=\int_{\Omega} I(\mathbf{x}) f_{\mathbf{X}}(\mathbf{x}) d \mathbf{x}
$$

Seja uma sequência decrescente de eventos $E_{i}$ tal que $E_{1} \supset E_{2} \supset \cdots \supset E_{m}=E$, de forma que:

$$
E_{k}=\bigcap_{i=1}^{k} E_{i}, \quad k=1, \ldots, m
$$

Como exemplo, para um sistema simples do tipo capacidade-demanda $(g(\mathbf{x})=C-D)$, a sequiência de eventos condicionais poderia ser obtida como:

$$
P\left[E_{i}\right]=P\left[C-D_{i}, \quad \operatorname{com} D_{1}>D_{2}>\cdots>D_{m}=D\right] .
$$

A partir da definição de probabilidades condicionais, pode-se mostrar que:

$$
P_{f}=P[E]=P\left[\bigcap_{i=1}^{m} E_{i}\right]=P\left[E_{1}\right] \cdot \prod_{i=2}^{m} P\left[E_{i} \mid E_{i-1}\right]
$$

Por exemplo, para $P\left[E_{1}\right]=0,1$ e $P\left[E_{i} \mid E_{i-1}\right]=0,1$ com $i=1,2,3$ isso leva a $P_{f}=$ $P[E]=10^{-4}$ o que é um valor baixo de probabilidade, porém como as probabilidades dos eventos intermediários são de 0,1 , uma amostra menor é requerida para cada subconjunto (AU; BECK, 2001).

Para obter o primeiro termo, $P\left[E_{1}\right]$, utiliza-se diretamente a Eq. (88), seja com a Amostragem Simples, com a Amostragem por Hipercubo Latino ou com a Amostragem por Variáveis Antitéticas. Para obter as probabilidades condicionais, $P\left[E_{i} \mid E_{i-1}\right]$, pode-se a princípio utilizar o Monte Carlo Bruto, desde que os pontos sejam simulados de acordo com o evento condicionante: 


$$
f_{\mathbf{X}}\left(\mathbf{x} \mid E_{i}\right)=\frac{f_{\mathbf{X}}(\mathbf{x}) I_{E_{i}}(\mathbf{x})}{P\left[E_{i}\right]}
$$

No entanto, esta técnica não é eficiente, em função do elevado número de pontos amostrais necessários para avaliar as probabilidades condicionais. Por outro lado, pode-se utilizar simulação por cadeias de Markov e o algoritmo de Metropolis-Hastings Modificados (AU; BECK, 2005).

Como a ideia básica desta técnica é estimar a probabilidade do evento falha $P[E]$ a partir de $P\left[E_{1}\right]$ e de $\left\{P\left[E_{i} \mid E_{i-1}\right] \mid i=2, \ldots, m\right\}$, os eventos intermediários $E_{i}$ são escolhidos de maneira adaptativa utilizando as informações de probabilidade dos eventos condicionais. Em confiabilidade estamos interessados em saber a probabilidade de um estado limite ser atingido. Assim pode-se escrever que:

$$
P_{f}=P[E]=P[\{g(\mathbf{X}) \leq 0\}] .
$$

Dado que o evento Falha é definido por:

$$
E=\{g(\mathbf{X}) \leq 0\}
$$

Assim, os eventos intermediários ficam definidos por:

$$
E_{i}=\left\{g(\mathbf{X}) \leq b_{i}\right\}
$$

Com $i=1, \ldots, m$, a sequência de eventos intermediários $E_{i}$ fica então definida pelo conjunto de limites de falha intermediários $\boldsymbol{b}=\left\{b_{1}, \ldots, b_{m}\right\}$. Por conveniência, as probabilidades de falhas condicionais são pré-estabelecidas, tal que $P\left[E_{i} \mid E_{i-1}\right]=P_{0}$.

Na execução da técnica, inicialmente é realizada uma simulação de Monte Carlo Bruto com um número $N_{S S}$ de amostras pré-estabelecido (e.g. $N_{S S}=500$ ), obtendo assim a amostra $X_{0, k}, \operatorname{com} k=1, \ldots, N_{S S}$. Posteriormente aplica-se a amostra $X_{0, k}$ na equação de estado limite, tal que, $Y_{0, k}=g\left(X_{0, k}\right)$. Então, o vetor $Y_{0, k}$ é ordenado em ordem crescente, obtendo-se o vetor $Y_{0, k}^{+}$. O limite de falha intermediário $b_{1}$ é determinado como o ponto amostral $Y_{0, k}^{+}$com $k=P_{0} N_{S S}$, dessa forma, $P\left[\left\{Y_{0, P_{0} N_{S S}}^{+} \leq b_{1}\right\}\right]=P_{0}$. Assim, existem $P_{0} N_{S S}$ pontos amostrais dentro do domínio de falha definido pelo limite de falha $b_{1}$. A partir de cada um desses pontos amostrais são geradas, via Cadeias de Markov pelo algoritmo de Metropolis-Hastings Modificado, $\left(1-P_{0}\right) N_{S S}$ pontos amostrais condicionais $X_{(1 \mid 0), k}$, com distribuição $p\left(\cdot \mid E_{1}\right)$ no primeiro nível, o que representa uma amostra de tamanho $N_{S S}$ no nível 1. Posteriormente aplicam-se os pontos amostrais na equação de estado limite, tal que $Y_{(1 \mid 0), k}=g\left(X_{(1 \mid 0), k}\right)$. 
Então, o vetor $Y_{(1 \mid 0), k}$ é ordenado em ordem crescente, obtendo-se o vetor $Y_{(1 \mid 0), k}^{+}$e o limite de falha intermediário $b_{2}$. Esse limite de falha intermediário é determinado como o ponto amostral $Y_{(1 \mid 0), k}^{+} \operatorname{com} k=P_{0} N_{S S}$, dessa forma, $P\left[\left\{Y_{(1 \mid 0), P_{0} N_{S S}}^{+} \leq b_{2}\right\}\right]=P_{0}$. Assim, o próximo evento $E_{2}=\left\{\mathbf{Y} \leq b_{2}\right\}$ é definido. É fácil notar que $P\left[E_{2} \mid E_{1}\right]=P\left[\left\{\mathbf{Y} \leq b_{2} \mid \mathbf{Y} \leq b_{1}\right\}\right]=P_{0}$. Assim, os $P_{0} N_{S S}$ pontos amostrais condicionais serão as sementes das amostras condicionais dos eventos seguintes. Assim, repetindo o processo, podem-se gerar amostras condicionais até que o limite final $b_{m}$ seja atingido, de forma que $b_{m}=0$ (AU, 2005). Este processo é ilustrado na Figura 23.

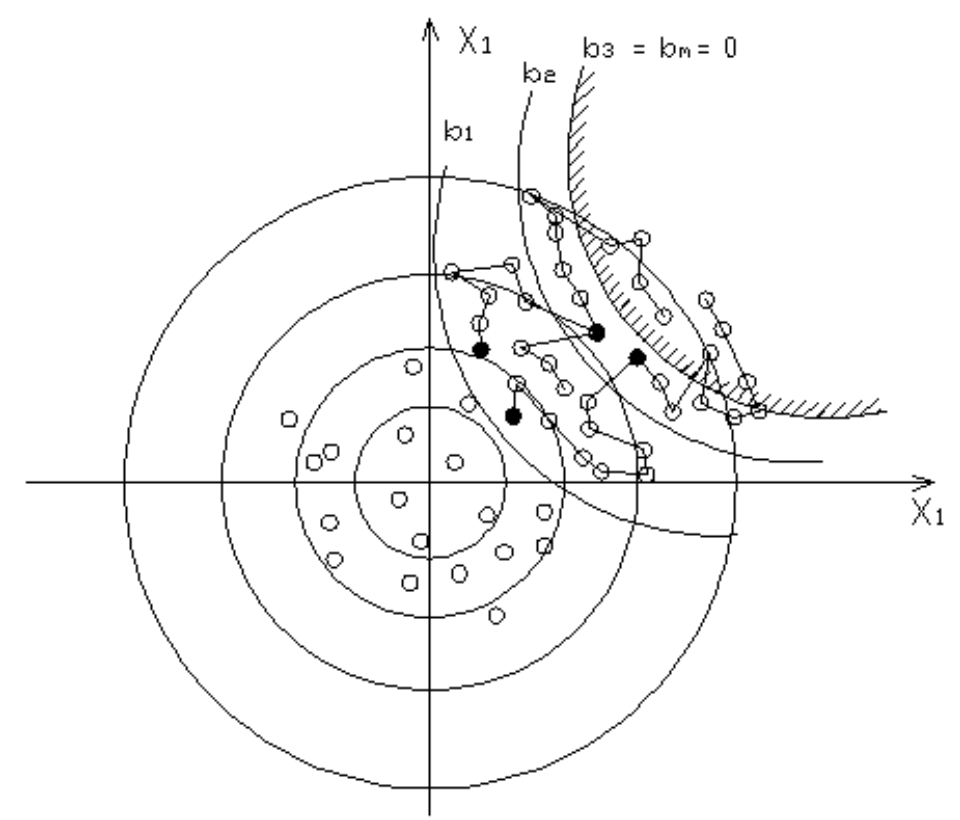

Figura 23. Geração de subconjuntos via cadeias de Markov.

A seguir serão apresentados como é realizada a estimativa do coeficiente de variação da probabilidade de falha e como se dá o processo de geração de amostras por cadeias de Markov utilizando o algoritmo de Metropolis-Hastings Modificado.

\subsubsection{Coeficiente de Variação da probabilidade de falha}

Nesta seção será apresentada como é feita a estimativa do coeficiente de variação da probabilidade de falha para a Simulação de Subconjuntos. As equações a serem apresentadas nesta seção foram introduzidas por (AU; BECK, 2001). Dado $P_{1}=P\left[E_{1}\right], P_{i}=P\left[E_{i} \mid E_{i-1}\right]$ e $P_{f}=P[E]$ com seus estimadores dados por $\hat{P}_{1}, \hat{P}_{i}$ e $\hat{P}_{f}$, respectivamente. De acordo com Au e Beck (2001), o coeficiente de variação $\delta_{1}$ do primeiro subconjunto, que não é condicional, é dado por: 


$$
\delta_{1}=\sqrt{\frac{1-P_{1}}{P_{1} N_{S S}}} .
$$

No caso das probabilidades de falha condicionais o coeficiente de variação $\delta_{i}$

$$
\delta_{i}=\sqrt{\frac{1-P_{i}}{P_{i} N_{S S}}\left(1+\gamma_{i}\right)},
$$

onde $\gamma_{i}$ é o fator de correlação relativo ao subconjunto $i$. Dessa forma, Au e Beck (2001), afirmam que embora $\hat{P}_{i}$ sejam correlacionados, simulações mostram que uma boa aproximação para o coeficiente de variação $\delta$ de $P_{f}$ é dada por:

$$
\delta^{2}=\sum_{i=1}^{m} \delta_{i}^{2}
$$

onde $m$ é o número de subconjuntos. Mais informações são apresentadas em (AU e BECK, 2001).

\subsubsection{Geração de Cadeias de Markov}

Um processo estocástico pode ser definido como a família de funções $X(\omega, t)$, onde $\omega$ é um ponto amostral do espaço amostral $\Omega$, que é associada a cada um destes pontos amostrais e é função de um parâmetro $t$, que em problemas físicos pode ser interpretado de diversas formas (e.g. tempo) (NOGUEIRA, 2009).

Os processos estocásticos podem ser classificados como:

a) Em relação ao estado: Discreto (Cadeias) ou Contínuo (Sequências);

b) Em relação ao parâmetro $t$ : Discreto ( $t$ é finito e contável) ou Contínuo ( $t$ infinito ou finito incontável).

Neste trabalho será dada ênfase aos processos Markovianos, que por sua vez levam a definição das cadeias de Markov.

Um processo estocástico é dito ser um Processo Markoviano se a probabilidade condicional de um evento futuro depende apenas do evento presente, ou seja, independe de eventos passados, tal que: 


$$
\begin{array}{r}
P\left\{X\left(t_{k+1}\right) \leq x_{k+1} \mid X\left(t_{k}\right)=x_{k}, \ldots, X\left(t_{0}\right)=x_{0}\right\} \\
=P\left\{X\left(t_{k+1}\right) \leq x_{k+1} \mid X\left(t_{k}\right)=x_{k}\right\}
\end{array}
$$

Tal processo é dito sem memória, pois eventos passados são desprezados de forma que interessam apenas as probabilidades de transição $P\left\{X\left(t_{k+1}\right) \leq x_{k+1} \mid X\left(t_{k}\right)=x_{k}\right\}$. Quando o espaço de estados é discreto diz-se que o processo Markoviano é uma Cadeia de Markov. Neste caso tem-se que:

$$
\begin{aligned}
P\{X(k+1)= & \left.x_{k+1} \mid X(k)=x_{k}, \ldots, X(0)=x_{0}\right\} \\
& =P\left\{X(k+1)=x_{k+1} \mid X(k)=x_{k}\right\} .
\end{aligned}
$$

O método de Monte Carlo com Cadeias de Markov é utilizado para simular amostras de variáveis aleatórias. Nele as amostras são simuladas como estados da cadeia de Markov e possuem uma distribuição alvo como sua distribuição limitante. Segundo Au e Beck (2001), a ideia por trás da aplicação do algoritmo de Metropolis-Hastings é que se é possível gerar uma amostra com distribuição condicional $q\left(\cdot \mid E_{i}\right)$, então, pode-se simular, como o próximo estado, uma nova amostra que também tenderá a ser distribuída de acordo com $q\left(\cdot \mid E_{i}\right)$.

O objetivo do algoritmo de Metropolis-Hastings é simular uma cadeia de Markov a partir de um ponto amostral, tal que a distribuição estacionária da Cadeia de Markov é $q\left(\cdot \mid E_{i}\right)=q\left(X_{i}\right) I_{E_{1}}\left(X_{i}\right) / P\left(E_{1}\right)$. Au e Beck (2001) utilizam a versão modificada do Algoritmo de Metropolis-Hastings, pois a versão original do algoritmo apresenta dificuldades em problemas de dimensão elevada. O algoritmo de Metropolis-Hastings modificado é apresentado a seguir (ZUEV, 2010):

1. Gere estados candidatos $y$

Para cada $j=1, \ldots, d$

a) Simule: $\hat{y}^{j} \sim S_{j}\left(\cdot \mid X_{n}^{j}\right)$;

b) Calcule a probabilidade de aceitação:

$$
\alpha^{j}\left(X_{n}^{j}, \hat{y}^{j}\right)=\min \left\{1, \frac{q_{j}\left(\hat{y}^{j}\right)}{q_{j}\left(X_{n}^{j}\right)}\right\}
$$

c) Aceite/Rejeite $y_{u}^{j}$, tal que:

$$
y^{j}=\left\{\begin{array}{c}
\hat{y}^{j} \text { com probabilidade } \alpha^{j}\left(X_{n}^{j}, \hat{y}^{j}\right) \\
x_{u}^{j} \text { com probabilidade } 1-\alpha^{j}\left(X_{n}^{j}, \hat{y}^{j}\right)
\end{array} .\right.
$$

2. Aceite/Rejeite $y$, tal que: 


$$
X_{n+1}=\left\{\begin{array}{l}
y \text { se } y \in E_{i} \\
X_{n} \text { se } y \notin E_{i}
\end{array}\right.
$$

Onde $d$ corresponde à dimensão do problema, $X_{n}^{j}$ corresponde ao estado inicial, $S_{j}(\cdot \mid \cdot)$ são as distribuições invariantes proposta, $q_{j}(\cdot)$ são as funções de distribuição de probabilidade marginais de $X^{j}$.

Segundo Zuev et al. (2012) existem valores ótimos do desvio padrão da distribuição proposta $\sigma^{*}$ e de $P_{0}$, que levam a um menor coeficiente de variação da probabilidade de falha. Assim, o melhor $\sigma^{*}$ é aquele que leva a uma taxa de aceitação em torno de $25 \%$ e o melhor $P_{0}$ se localiza entre 0,1 e 0,3. Porém, a escolha de tais parâmetros ainda é algo bem subjetivo e deve ser feita com cuidado. Segundo Au e Beck (2001), o desvio padrão da distribuição proposta $\sigma^{*}$ afeta o tamanho da região coberta pela amostra gerada pelas cadeias de Markov, consequentemente controlando a eficiência da técnica. Assim, uma amostra pouco espalhada tende a diminuir a convergência do estimador da probabilidade de falha, uma vez que os pontos amostrais são gerados muito próximos uns dos outros. Por outro lado, uma amostra excessivamente espalhada tende a diminuir a taxa de aceitação dos pontos amostrais simulados e consequentemente reduzindo a convergência do estimador da probabilidade de falha, uma vez que mais amostras repetidas são geradas. Ainda assim, $\sigma_{i}^{*}$ pode ser calculado como uma parcela do desvio padrão $\sigma_{i}$ da $i$-ésima variável aleatória. 


\section{EXEMPLOS}

Neste capítulo são estudados exemplos com a aplicação das técnicas de amostragem inteligente em problemas de confiabilidade de estruturas independentes do tempo. $\mathrm{O}$ estudo a ser realizado consiste na análise comparativa da convergência da probabilidade de falha $\left(P_{f}\right)$ com o aumento do tamanho da amostra $(N)$, da convergência do coeficiente de variação da probabilidade de falha (C.V. da $\left.P_{f}\right)$ com o aumento do tamanho da amostra $(N)$ e do tempo de processamento com o aumento do tamanho da amostra $(N)$. São aplicadas as seguintes técnicas:

○ Monte Carlo Bruto (BRUTO) com:

- Amostragem Simples (SIMC)

- Amostragem por Hipercubo Latino (SLHS)

- Amostragem por Variáveis Antitéticas (ASIMC)

○ Amostragem por Importância (AI) com:

- Amostragem Simples (ISMC)

- Amostragem por Hipercubo Latino (ILHS)

- Amostragem por Variáveis Antitéticas (AISMC)

○ Amostragem Assintótica (AA) com:

- Amostragem Simples (ASMC)

- Amostragem por Hipercubo Latino (ALHS)

- Amostragem por Variáveis Antitéticas (AASMC)

- Amostragem Melhorada (AM) com:

- Amostragem Simples (AM)

- Amostragem por Hipercubo Latino (AM_LHS)

- Amostragem por Variáveis Antitéticas (AAM)

○ Simulação de Subconjuntos (SS) com:

- Amostragem Simples (SS)

- Amostragem por Hipercubo Latino (SSLHS)

- Amostragem por Variáveis Antitéticas (SSA)

O estudo comparativo da convergência não é aplicado à técnica de Simulação de Subconjuntos, pois, a qualidade de sua resposta não é caracterizada de maneira adequada 
apenas aumentando o tamanho da amostra em cada subconjunto. Deve-se levar em consideração que esta técnica é adaptativa. Assim, sua resposta depende da probabilidade de falha intermediária (condicional) $P_{0}$, do desvio-padrão do caminhante aleatório, do tipo de distribuição de probabilidade do caminhante aleatório e do tamanho da amostra em cada subconjunto $\left(N_{S S}\right)$.

Pode-se destacar que a qualidade da resposta obtida pelas técnicas que fazem uso de ajuste de curva é também dependente de seus parâmetros, tais como o intervalo do parâmetro e o número de pontos de suporte. Porém, como estas técnicas normalmente precisam de uma amostra maior que aquela utilizada pela Simulação de Subconjuntos, estas serão avaliadas por gráficos de convergência, fixando tais parâmetros de forma que uma boa resposta seja obtida.

Nos problemas 1 a 5 é utilizado um computador Intel ${ }^{\circledR}$ Core $^{\mathrm{TM}}$ i7-3770 CPU 3,40 GHz 64 bits com 12 GB de memória RAM. Já no problema 6, utiliza-se um computador com processador Intel ${ }^{\circledR}$ Core $^{\mathrm{TM}}$ i5-2500 CPU 3,30 GHz 64 bits com 4 GB de memória RAM.

A seguir serão apresentados os exemplos que são estudados neste trabalho.

\subsection{Exemplo 1: Capacidade-Demanda}

Este problema consiste em estimar a probabilidade de a demanda (D) exceder a capacidade $(C)(C<D)$, quando as distribuições de probabilidade das variáveis aleatórias $\mathrm{C}$ e D são conhecidas. A equação de estado limite deste problema é linear (Eq. (133)).

$$
g(\mathrm{C}, \mathrm{D})=\mathrm{C}-\mathrm{D}=0
$$

Os parâmetros das distribuições de probabilidade de cada variável aleatória são apresentados na Tabela 1.

Tabela 1. Médias e desvios padrão das variáveis aleatórias do Exemplo 1.

\begin{tabular}{clll}
\hline Variável Aleatória & Distribuição & Média & Desvio Padrão \\
\hline C & Normal & 2,0 & 0,20 \\
D & Normal & 1,0 & 0,15 \\
\hline
\end{tabular}

Considerando todas variáveis aleatórias independentes e com distribuição normal de probabilidade, este problema pode ser resolvido analiticamente, tal como:

$$
\beta=\frac{\mu_{\mathrm{C}}-\mu_{\mathrm{D}}}{\sqrt{\sigma_{\mathrm{C}}^{2}+\sigma_{\mathrm{D}}^{2}}}=\frac{2-1}{\sqrt{0,2^{2}+0,15^{2}}} \cong 4
$$


Este valor de $\beta$ corresponde a uma probabilidade de falha $\mathrm{P}_{\mathrm{f}}=\Phi(-\beta)=3,1671 \times 10^{-5}$. Tal valor será a probabilidade de falha de referência nas análises comparativas.

\subsubsection{Amostragem Assintótica}

Nesta seção é verificado através dos gráficos de ajuste, como a Amostragem Assintótica se adequa ao problema. O objetivo é demonstrar que o resultado do ajuste melhora à medida que se aumenta o tamanho da amostra. São utilizadas amostras de tamanho $2 \times 10^{3}$ (Figura 24) e $1 \times 10^{5}$ (Figura 25). Utiliza-se a Amostragem Simples, a Amostragem por Hipercubo Latino e a Amostragem por Variáveis Antitéticas. Estes tamanhos de amostra são escolhidos como o intervalo inferior e superior do estudo da convergência, que será apresentado em 7.1.4. O parâmetro $f$ é variado de 0,4 a 0,6 . Utilizam-se 5 pontos de suporte.

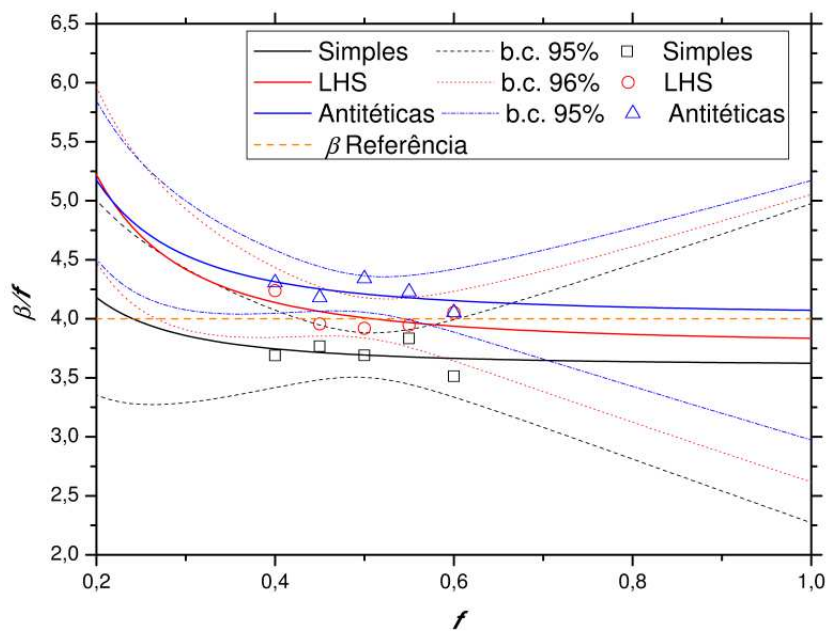

Figura 24. Regressão não linear utilizada na Amostragem Assintótica para amostras de tamanho $2 \times 10^{3}$, no Exemplo 1.

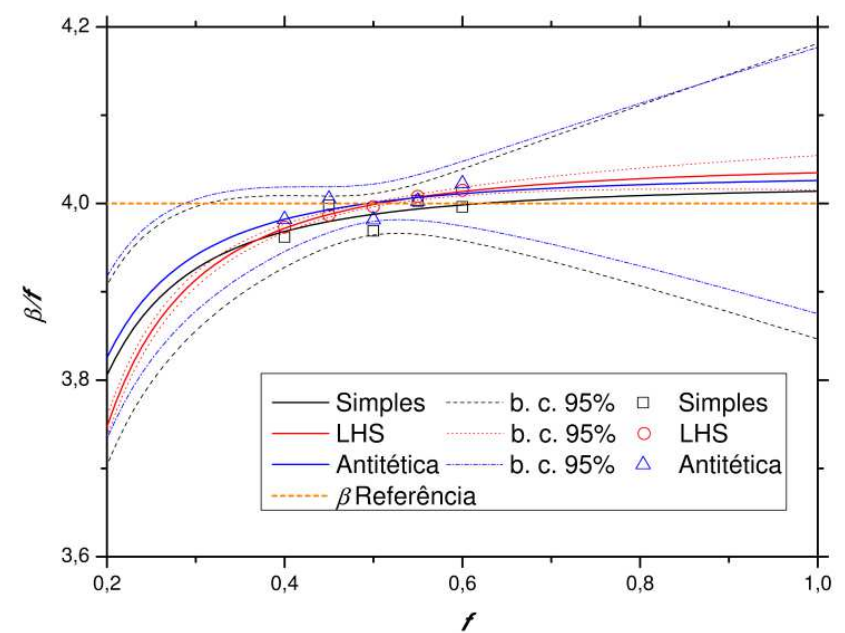

Figura 25. Regressão não linear utilizada na Amostragem Assintótica para amostras de tamanho $1 \times 10^{5}$, no Exemplo 1. 
Os parâmetros ajustados na técnica em questão são apresentados na Tabela 2, para a Amostragem Simples (ASMC), para a Amostragem por Hipercubo Latino (ALHS) e para a Amostragem por Variáveis Antitéticas (AASMC). Neste caso, o valor do parâmetro $C$ foi fixado em -2,0, pois esse valor se mostrou mais adequado na resolução deste problema uma vez que as variáveis aleatórias possuem caudas bem comportadas.

Tabela 2. Parâmetros ajustados na Amostragem Assintótica, para o Exemplo 1.

\begin{tabular}{ccccccc}
\hline $\boldsymbol{N}$ & \multicolumn{3}{c}{$\mathbf{2 \times 1 0}^{\mathbf{3}}$} & $\boldsymbol{B}$ & $\mathbf{1 \times 1 \mathbf { 1 0 } ^ { \mathbf { 5 } }}$ \\
\hline & $\boldsymbol{A}$ & $\boldsymbol{B}$ & $\boldsymbol{C}$ & $\boldsymbol{A}$ & $\boldsymbol{B}$ & $\boldsymbol{C}$ \\
ASMC & 3,6004 & $2,3151 \times 10^{-2}$ & $-2,0000$ & 4,0225 & $-8,6745 \times 10^{-3}$ & $-2,0000$ \\
ALHS & 3,7773 & $5,7738 \times 10^{-2}$ & $-2,0000$ & 4,0468 & $-1,1986 \times 10^{-2}$ & $-2,0000$ \\
AASMC & 4,0262 & $4,5983 \times 10^{-2}$ & $-2,0000$ & 4,0344 & $-8,3602 \times 10^{-3}$ & $-2,0000$ \\
\hline
\end{tabular}

Realizando um maior número de simulações, observa-se uma melhor concordância entre a curva ajustada e os pontos de suporte, como pode ser observado na Figura 24 e na Figura 25.

A probabilidade de falha e seu coeficiente de variação, para diferentes tamanhos de amostra, são apresentados na Tabela 3.

Tabela 3. Probabilidade de falha e coeficiente de Variação da probabilidade de falha, para a Amostragem Assintótica, no Exemplo 1.

\begin{tabular}{|c|c|c|c|c|}
\hline \multicolumn{5}{|c|}{$P_{f}$ de referência: $3,1671 \times 10^{-5}$} \\
\hline \multirow[t]{2}{*}{$N$} & \multicolumn{2}{|c|}{$2 \times 10^{3}$} & \multicolumn{2}{|c|}{$1 \times 10^{5}$} \\
\hline & $P_{f}$ & $C . V$. & $P_{f}$ & C.V. \\
\hline ASMC & $1,453 \times 10^{-4}$ & 40,0892 & $2,988 \times 10^{-5}$ & 0,5133 \\
\hline ALHS & $6,280 \times 10^{-5}$ & 35,5488 & $2,733 \times 10^{-5}$ & 0,0441 \\
\hline AASMC & $2,329 \times 10^{-5}$ & 31,7296 & $2,838 \times 10^{-5}$ & 0,4479 \\
\hline
\end{tabular}

Vale ressaltar que tal resultado depende do intervalo que se adota para o parâmetro $f$. Maiores estudos referentes a este intervalos podem ser realizados, mas não são objetivos deste trabalho.

\subsubsection{Amostragem Melhorada}

Na Figura 26 e na Figura 27, se observam as curvas ajustada aos pontos de suporte para amostras de tamanho $2 \times 10^{3}$ e $1 \times 10^{5}$, respectivamente. Estes tamanhos de amostra são escolhidos como o intervalo inferior e superior do estudo da convergência, que será apresentado em 7.1.4. Nesse caso, são utilizados 100 pontos de suporte, com $\lambda$ variando de 0,4 a 0,9 . 


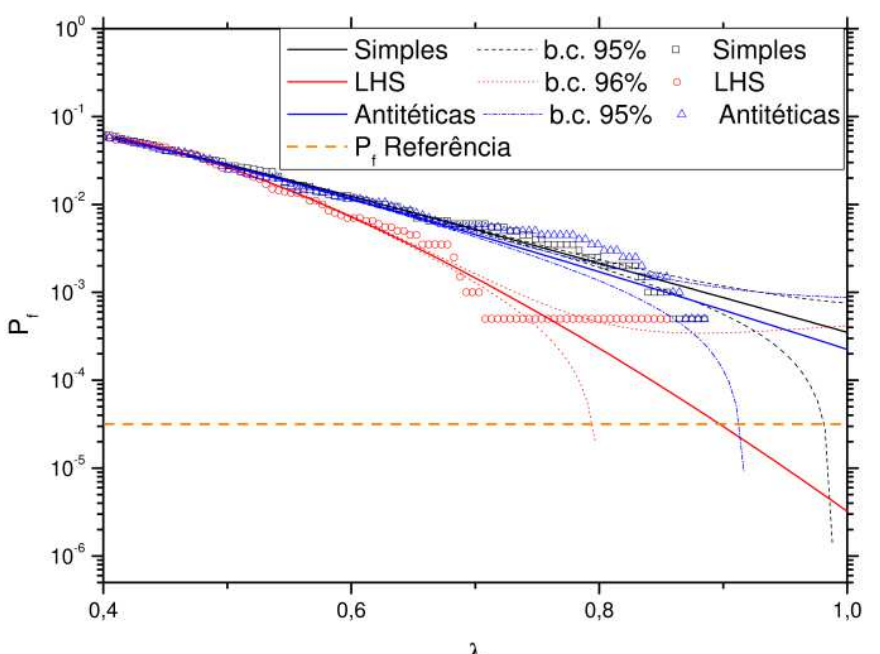

Figura 26. Regressão não linear utilizada na Amostragem Melhorada para amostras de tamanho $2 \times 10^{3}$, no Exemplo 1.

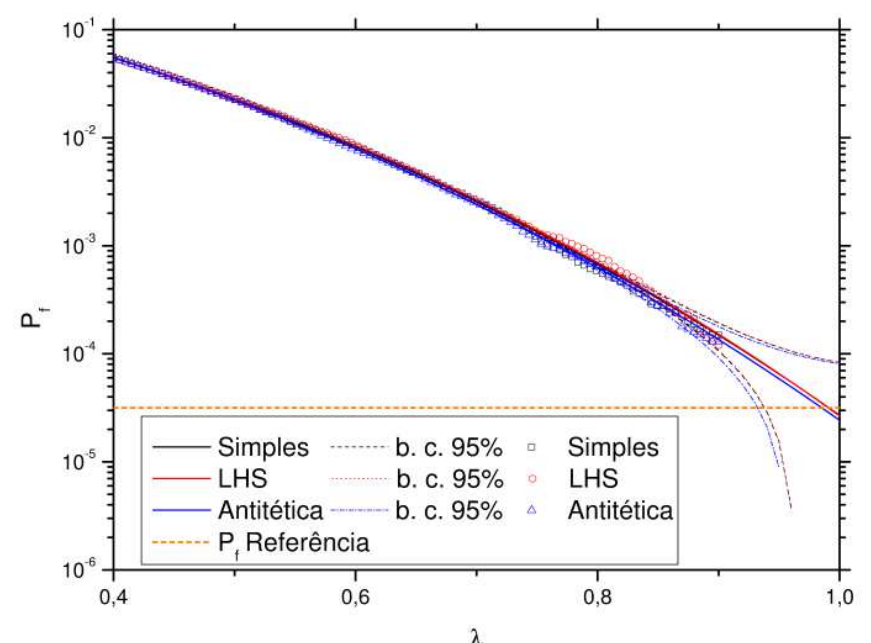

Figura 27. Regressão não linear utilizada na Amostragem Melhorada para amostras de tamanho $1 \times 10^{5}$, no Exemplo 1.

$\mathrm{Na}$ Figura 26 e na Figura 27, se observa que o aumento do tamanho da amostra leva a uma redução da largura da banda de confiança do ajuste, consequentemente do intervalo de confiança da probabilidade de falha estimada.

Os parâmetros ajustados na técnica em questão são apresentados na Tabela 4, para uma amostra de tamanho $2 \times 10^{3}$ e na Tabela 5 para uma amostra de tamanho $1 \times 10^{5}$. Utiliza-se a Amostragem Simples (AM), a Amostragem por Hipercubo Latino (AM_LHS) e a Amostragem por Variáveis Antitéticas (AAM). 
Tabela 4. Parâmetros ajustados na Amostragem Melhorada, para uma amostra de tamanho $2 \times 10^{3}$, no Exemplo 1.

\begin{tabular}{ccccc}
\hline & $\boldsymbol{q}$ & $\boldsymbol{a}$ & $\boldsymbol{b}$ & $\boldsymbol{c}$ \\
\hline AM & $6,2624 \times 10^{-2}$ & 8,8961 & 0,4000 & 1,0574 \\
AM_LHS & $5,8522 \times 10^{-2}$ & 20,118 & 0,39785 & 1,4166 \\
AAM & $5,8786 \times 10^{-2}$ & 9,8633 & 0,4000 & 1,1196 \\
\hline
\end{tabular}

Tabela 5. Parâmetros ajustados na Amostragem Melhorada, para uma amostra de tamanho $1 \times 10^{5}$, no Exemplo 1 .

\begin{tabular}{ccccc}
\hline & $\boldsymbol{q}$ & $\boldsymbol{a}$ & $\boldsymbol{b}$ & $\boldsymbol{c}$ \\
\hline AM & 5,4913 & $1,8035 \times 10^{-2}$ & $-2,5018$ & 5,2015 \\
AM_LHS & 2,8971 & $7,8199 \times 10^{-2}$ & $-1,9209$ & 4,6638 \\
AAM & 4,0108 & 0,20702 & $-1,6978$ & 4,0921 \\
\hline
\end{tabular}

A Tabela 6 apresenta os valores das probabilidades de falha e de seus coeficientes de variação para diferentes tamanhos da amostra.

Tabela 6. Probabilidade de falha e coeficiente de Variação da probabilidade de falha, para a Amostragem Melhorada, no Exemplo 1.

\begin{tabular}{|c|c|c|c|c|}
\hline \multicolumn{5}{|c|}{$P_{f}$ de referência: $3,1671 \times 10^{-5}$} \\
\hline$N$ & \multicolumn{2}{|c|}{$2 \times 10^{3}$} & \multicolumn{2}{|c|}{$1 \times 10^{5}$} \\
\hline & $P_{f}$ & C.V. & $P_{f}$ & C.V. \\
\hline ASMC & $3,513 \times 10^{-4}$ & 0,5838 & $2,697 \times 10^{-5}$ & 1,0690 \\
\hline ALHS & $3,224 \times 10^{-6}$ & 65,5407 & $2,669 \times 10^{-5}$ & 1,0844 \\
\hline AASMC & $2,246 \times 10^{-5}$ & 1,4771 & $2,425 \times 10^{-5}$ & 1,1945 \\
\hline
\end{tabular}

\subsubsection{Simulação de Subconjuntos}

Na Simulação de Subconjuntos, a quantidade de subconjuntos é determinada de maneira adaptativa a partir do tamanho da amostra de cada subconjunto $\left(N_{S S}\right)$, da ordem de grandeza da probabilidade de falha $\left(P_{f}\right)$, do valor das probabilidades de falha intermediárias $\left(P_{0}\right)$, do tipo do caminhante aleatório e do modelo da equação de estado limite.

Neste trabalho, o desvio padrão do caminhante aleatório $i\left(\sigma_{i}^{\text {sim }}\right)$ é dado pelo produto de um fator $\alpha$ pelo desvio padrão da variáveis aleatória $i$ do problema. Assim, o desvio padrão do caminhante aleatório $i$, com $i=1, \ldots, n_{v a}$, onde $n_{v a}$ é o número de variáveis aleatórias, é dado por:

$$
\sigma_{i}^{s i m}=\alpha \cdot \sigma_{i}
$$


onde $\alpha$ é um valor maior que zero. No StRAnD, o usuário pode definir o valor do desvio padrão e o tipo de distribuição de probabilidade, do caminhante aleatório, de maneira direta ou através do parâmetro $\alpha$.

O caminhante aleatório utilizado na Simulação de Subconjuntos é definido pelos dados apresentados na Tabela 7.

Tabela 7. Médias e desvios padrão do caminhante aleatório do Exemplo 1.

\begin{tabular}{clll}
\hline Variável Aleatória & Distribuição & Média & Desvio padrão \\
\hline C & Uniforme & 0,0 & $\alpha \times 0,20$ \\
D & Uniforme & 0,0 & $\alpha \times 0,15$ \\
\hline
\end{tabular}

Na Figura 28 é realizada uma análise referente a variação do desvio padrão do caminhante aleatório. Neste caso, utiliza-se um caminhante aleatório com distribuição uniforme de probabilidade, $P_{0}=0,1$ e $N_{S S}=700$. Para gerar os pontos amostrais do primeiro subconjunto é utilizada a Amostragem Simples, mas este subconjunto pode ser criado por meio da Amostragem por Hipercubo Latino ou por meio da Amostragem por Variáveis Antitéticas. São obtidos 5 subconjuntos, e cada um deles é representado por simbolos e cores diferentes.

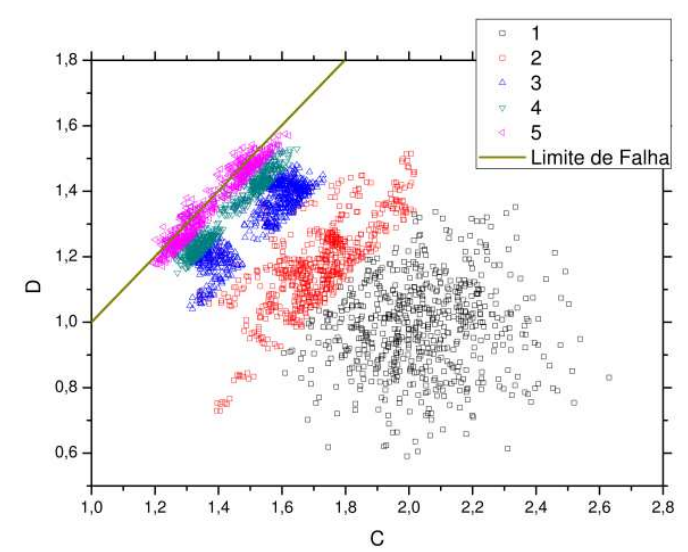

a) $\alpha=0,1$

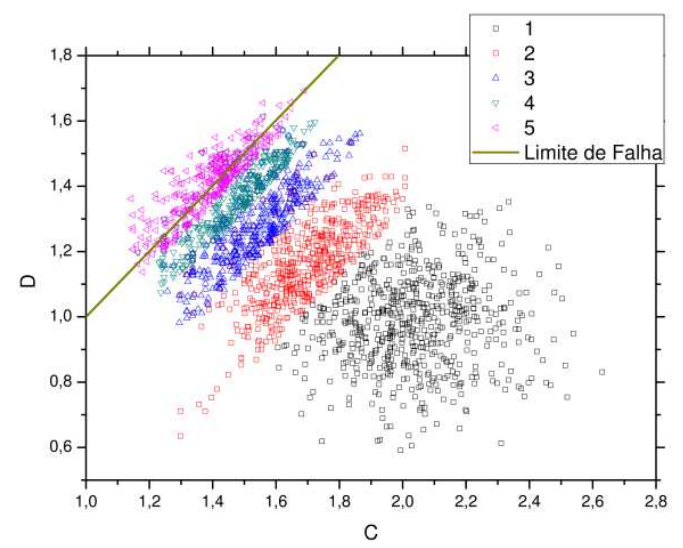

b) $\alpha=0,4$ 


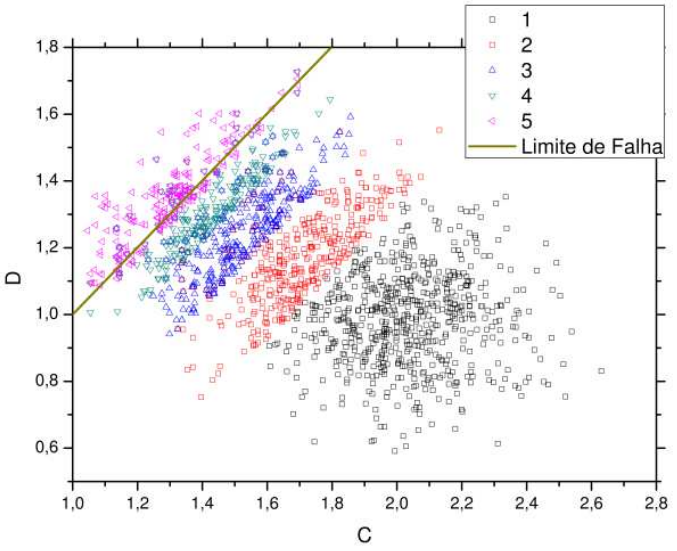

c) $\alpha=0,8$

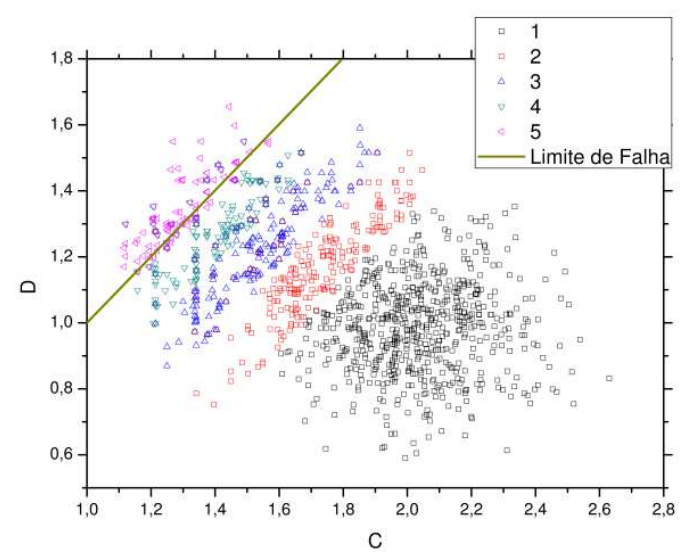

e) $\alpha=2,0$

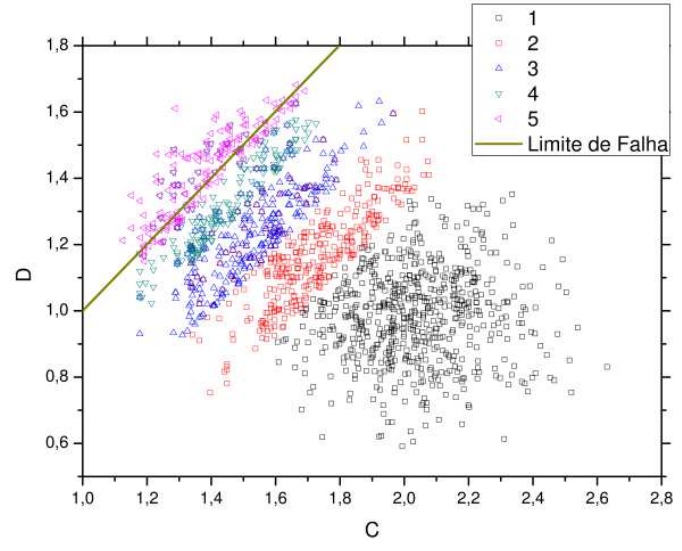

d) $\alpha=1,0$

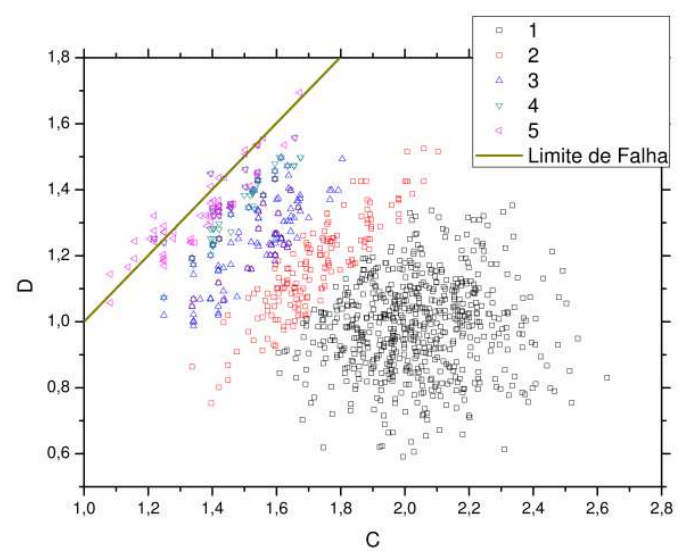

f) $\alpha=3,0$

Figura 28. Subconjuntos gerados na Simulação de Subconjuntos, para o Exemplo 1.

Observa-se que quando $\alpha=0,1$ (Figura 28a) ocorre uma alta taxa de aceitação dos pontos amostrais pelo algoritmo de Metropolis-Hastings Modificado. Quando $\alpha$ é maior que um, observa-se um aumento da rejeição dos pontos amostrais pelo algoritmo de MetropolisHastings Modificado.

Portanto se verifica uma boa adequação da Simulação de Subconjuntos na análise do problema em questão.

\subsubsection{Análise de convergência}

Realiza-se um comparativo entre o Monte Carlo Bruto, a Amostragem por Importância, a Amostragem Assintótica e a Amostragem Melhorada. Adota-se, em todas as técnicas citadas, o uso da Amostragem Simples, da Amostragem por Hipercubo Latino e da Amostragem por Variáveis Antitéticas. O tamanho da amostra é variado de $2 \times 10^{3}$ até $1 \times 10^{5}$, tomando-se nesse intervalo 100 pontos. 
a) Média

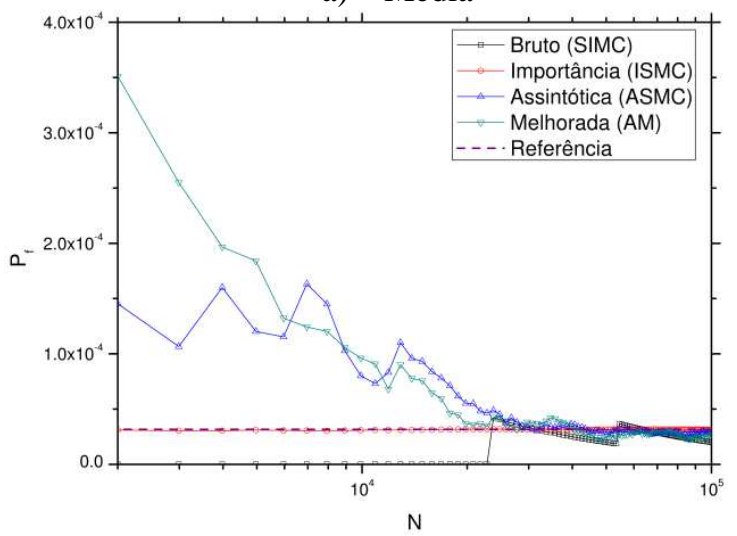

b) C.V.

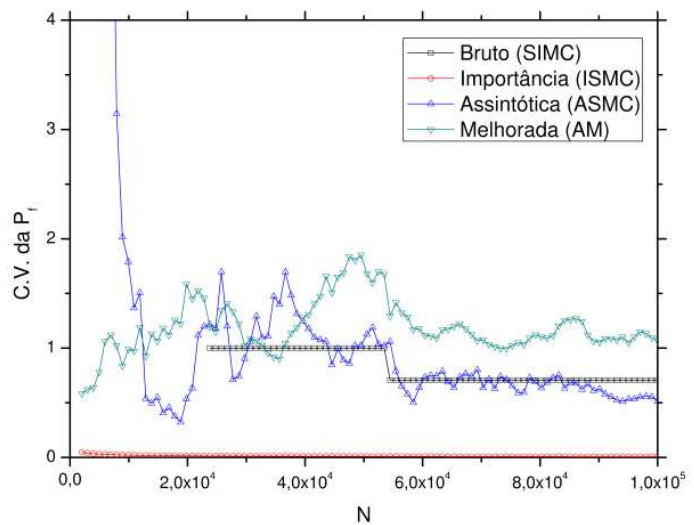

Figura 29. Comparativo da convergência da $P_{f}$ (a) e de C.V. (b) para a Amostragem Simples, no Exemplo 1.

a) Média

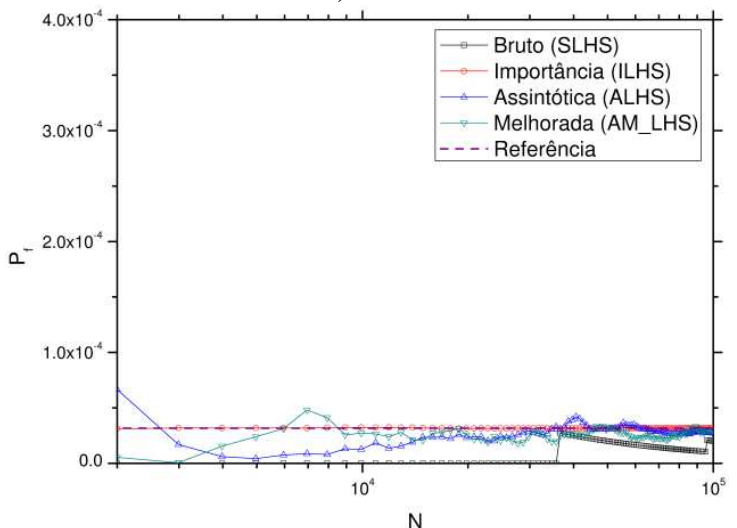

b) C.V.

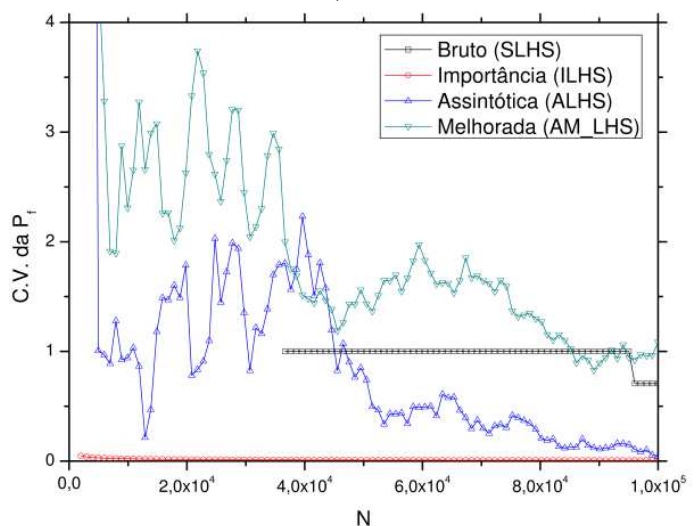

Figura 30. Comparativo da convergência da $P_{f}($ a) e de C.V. (b) para a Amostragem por Hipercubo Latino, no Exemplo 1.

a) Média

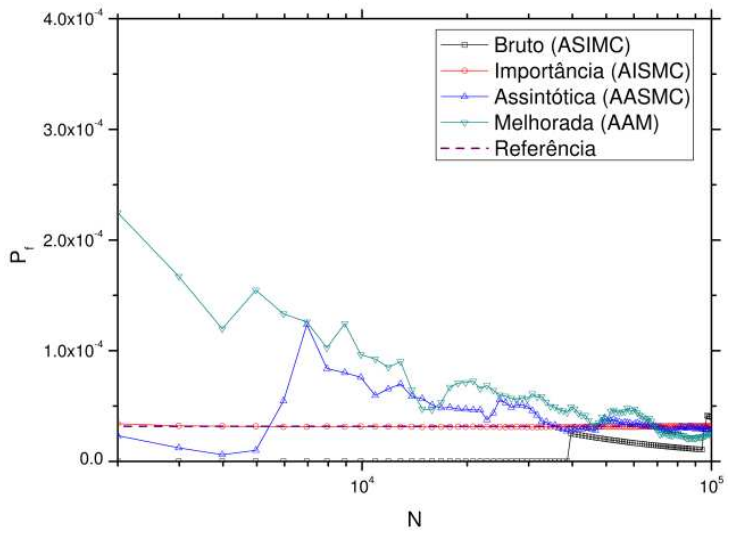

b) C.V.

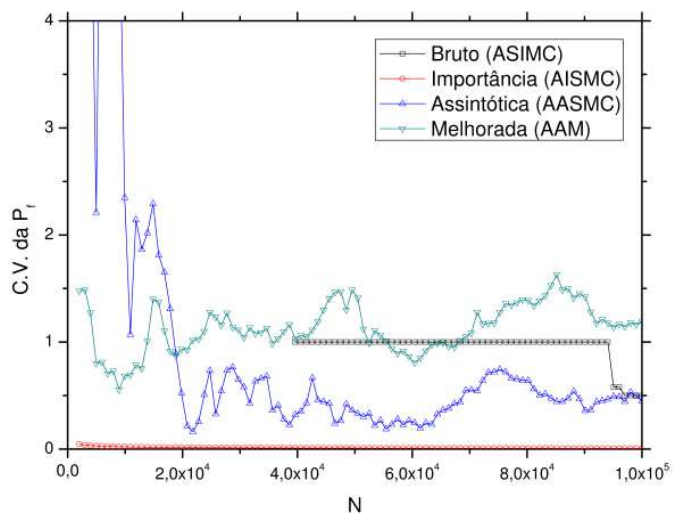

Figura 31. Comparativo da convergência da $P_{f}($ a) e de C.V. (b) para a Amostragem por Variáveis Antitéticas, no Exemplo 1.

Pode-se notar que as técnicas em análise tendem a convergir para o mesmo ponto, que é representado pela linha de referência da probabilidade de falha. A Amostragem por 
Importância apresenta o melhor resultado dentre as técnicas estudadas, pois apresenta uma rápida convergência da probabilidade de falha. As técnicas de Amostragem Assintótica e de Amostragem Melhorada apresentam uma nítida vantagem em relação ao Monte Carlo Bruto, pois, estas técnicas são capazes de estimar valores de probabilidades de falha com uma amostra pequena. Também é possível notar que a Amostragem Assintótica apresenta uma boa redução do coeficiente de variação da pobabilidade de falha, a medida que se aumenta o tamanho da amostra.

A seguir são apresentados os graficos do comparativo de cada técnica com aplicação de diferentes técnicas de amostragem básica. Neste caso é feito um gráfico para cada uma das técnicas (Monte Carlo Bruto, Amostragem por Importância, Amostragem Assintótica e Amostragem Melhorada) e para cada são obtidas três curvas, uma para cada técnica de amostragem básica (Amostragem Simples, Amostragem por Hipercubo Latino e Amostragem por Variáveis Antitéticas).

a) Média

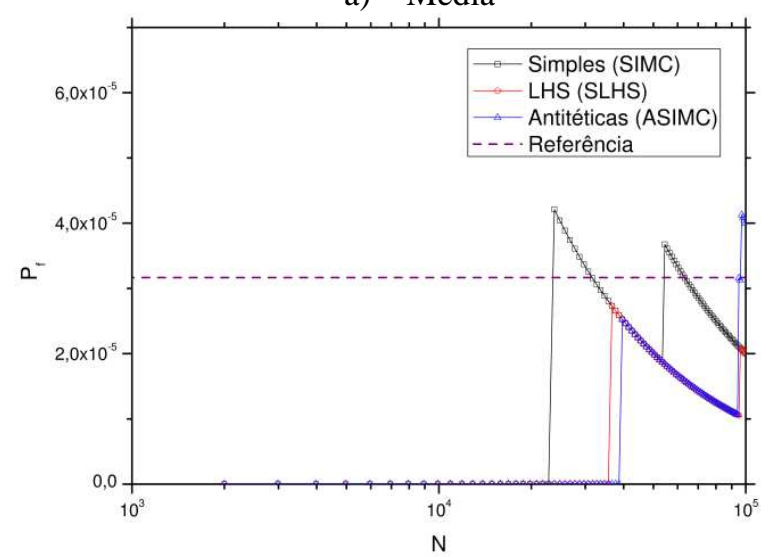

b) C.V.

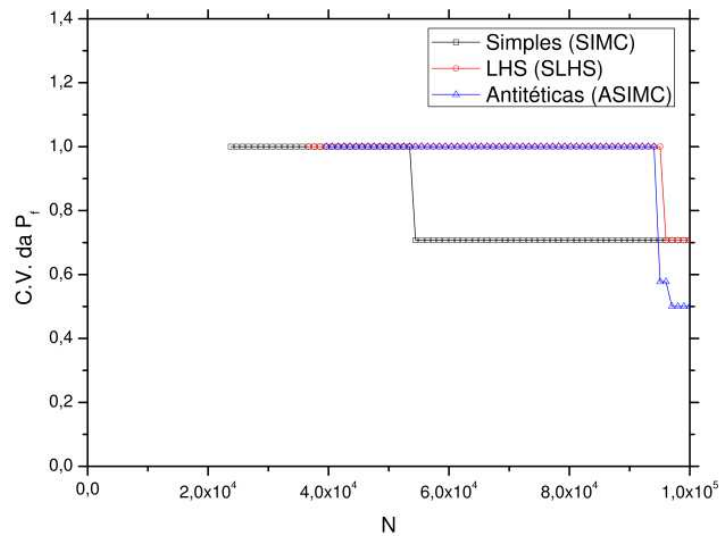

Figura 32. Comparativo da convergência da $P_{f}($ a) e de C.V. (b) para o Monte Carlo Bruto, no Exemplo 1.

a) Média

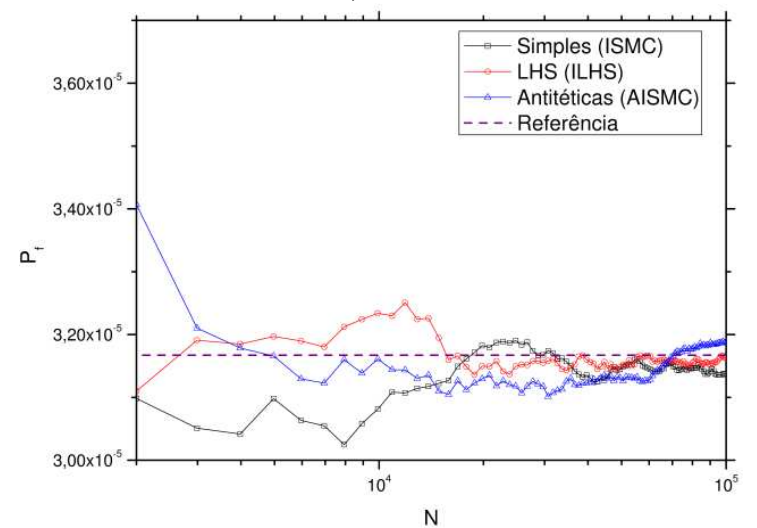

b) C.V.

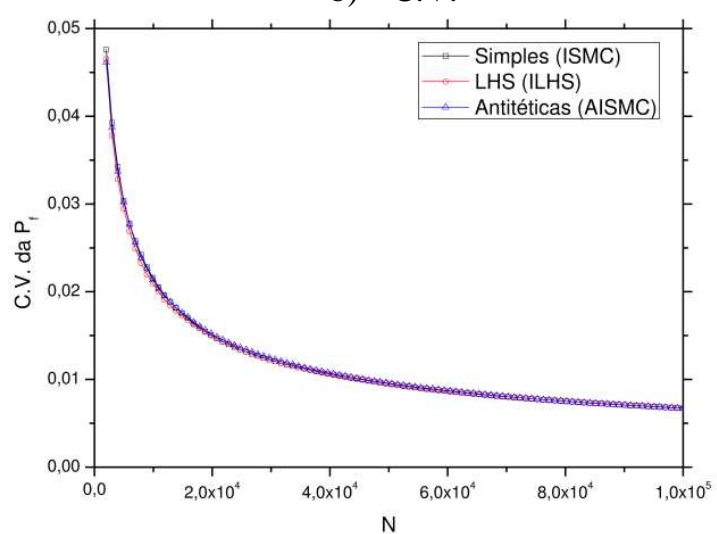

Figura 33. Comparativo da convergência da $P_{f}($ a) e de C.V. (b) para a Amostragem por Importância, no Exemplo 1. 
a) Média

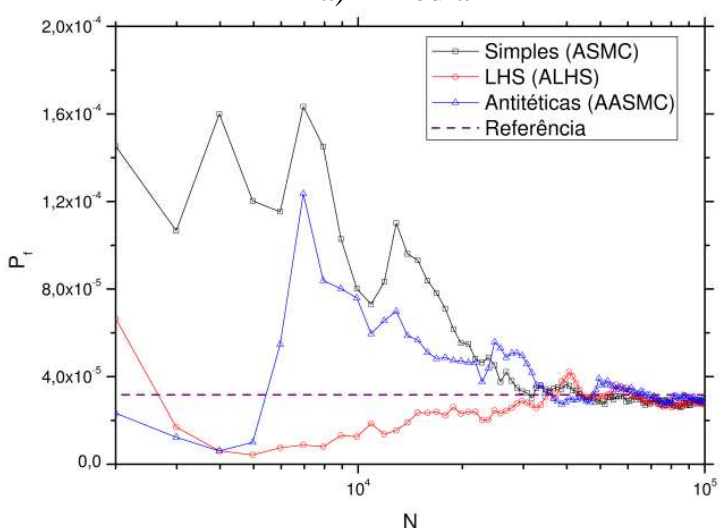

b) C.V.

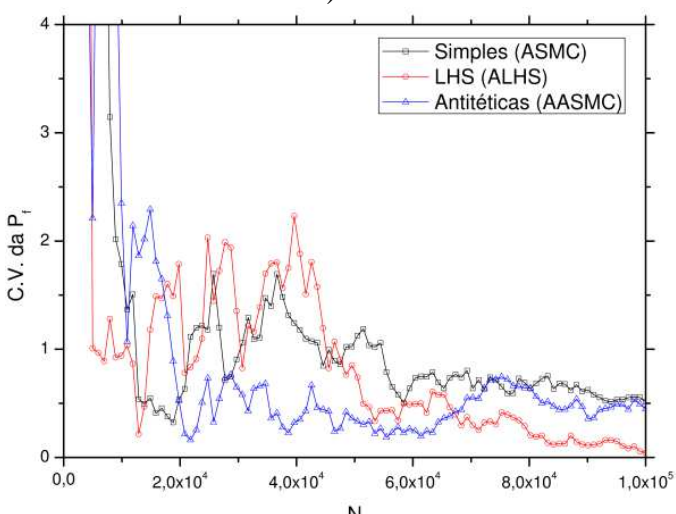

Figura 34. Comparativo da convergência da $P_{f}($ a) e de C.V. (b) para a Amostragem Assintótica, no Exemplo 1.

a) Média

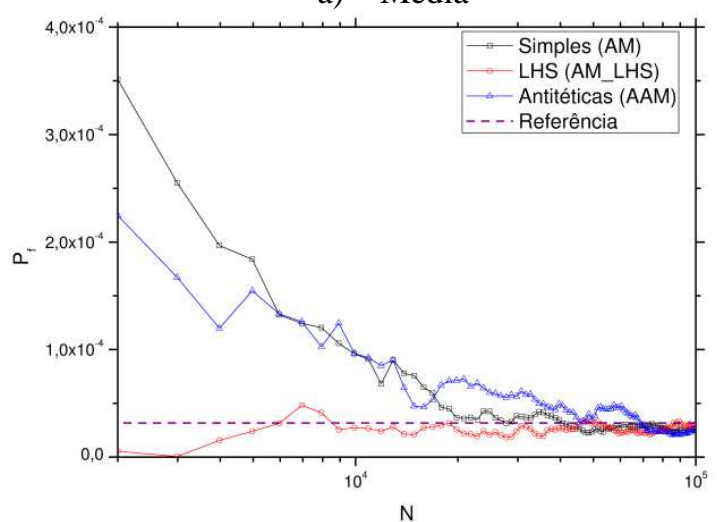

b) C.V.

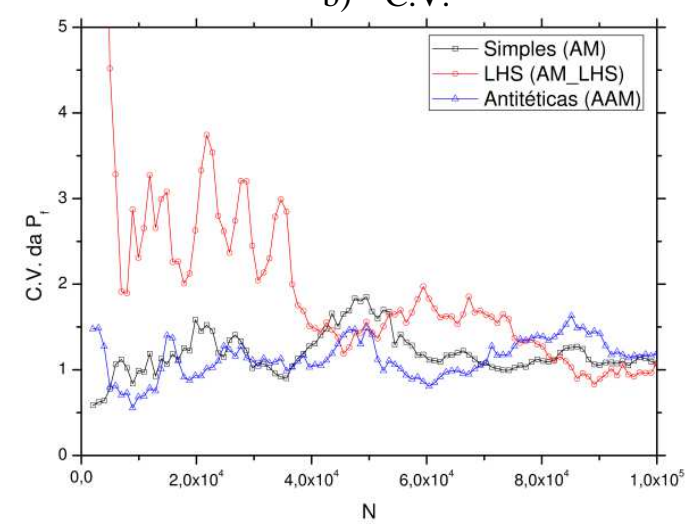

Figura 35. Comparativo da convergência da $P_{f}$ (a) e de C.V. (b) para a Amostragem Melhorada, no Exemplo 1.

Na Figura 32, observa-se que o método de Monte Carlo Bruto não apresenta boas estimativas da probabilidade de falha.

Na Figura 33, pode-se observar que o uso da Amostragem por Hipercubo Latino e da Amostragem por Variáveis Antitéticas são benéficas para a Amostragem por Importância, especialmente nos trechos iniciais dos gráficos de convergência, mas vale lembrar que essa diferença é pequena.

Na Figura 34, pode-se observar uma certa vantagem do uso da Amostragem Assintótica com o uso da Amostragem por Hipercubo Latino e da Amostragem por Variáveis Antitéticas, especialmente nos trechos iniciais, mesmo que isso não seja expresso no coeficiente de variação (Figura 34b). 
Na Figura 35 é visível a vantagem obtida, em termos de convergência da probabilidade de falha, pelo uso da Amostragem por Hipercubo Latino na Amostragem Melhorada.

\subsubsection{Comparativo da probabilidade de falha e de seu coeficiente de variação}

Neste exemplo será utilizada uma amostra de tamanho 9.200. Na técnica de simulação de subconjuntos foram adotados os seguintes parâmetros: $N_{S S}=2.000, \alpha=0,7$ e $P_{0}=0,1$. O comparativo dos resultados obtidos, para as técnicas estudadas neste trabalho e para uma amostra de tamanho 9.200, pode ser observado na Tabela 8.

Tabela 8. Comparativo da $P_{f}$ para uma amostra de tamanho 9.200, no Exemplo 1.

\begin{tabular}{|c|c|c|c|c|}
\hline \multicolumn{5}{|c|}{$P_{f}$ de referência: $3,1671 \times 10^{-5}$} \\
\hline \multicolumn{5}{|c|}{ SIMPLES } \\
\hline Sigla & Técnica & $\boldsymbol{P}_{\boldsymbol{f}}$ & C.V.da $P_{f}$ & $\operatorname{Erro}(\%)$ \\
\hline BRUTO & Bruto & -- & -- & -- \\
\hline AI & Importância & $3,057 \times 10^{-5}$ & 0,0224 & 3,48 \\
\hline $\mathbf{A A}$ & Assintótica & $9,771 \times 10^{-5}$ & 1,9634 & 208,52 \\
\hline $\mathbf{A M}$ & Melhorada & $9,186 \times 10^{-5}$ & 0,9926 & 190,04 \\
\hline SS & Subconjuntos & $4,895 \times 10^{-5}$ & 0,2242 & 54,56 \\
\hline \multicolumn{5}{|c|}{ HIPERCUBO LATINO } \\
\hline Sigla & Técnica & $\boldsymbol{P}_{\boldsymbol{f}}$ & C.V.da $P_{f}$ & \\
\hline BRUTO & Bruto & -- & -- & -- \\
\hline AI & Importância & $3,046 \times 10^{-5}$ & 0,0223 & 3,82 \\
\hline $\mathbf{A A}$ & Assintótica & $2,576 \times 10^{-5}$ & 72,5439 & 18,66 \\
\hline $\mathbf{A M}$ & Melhorada & $1,147 \times 10^{-5}$ & 9,2957 & 63,78 \\
\hline SS & Subconjuntos & $3,594 \times 10^{-5}$ & 0,2265 & 13,48 \\
\hline \multicolumn{5}{|c|}{ VARIÁVEIS ANTITÉTICAS } \\
\hline Sigla & Técnica & $\boldsymbol{P}_{f}$ & C.V.da $P_{f}$ & \\
\hline BRUTO & Bruto & -- & -- & -- \\
\hline AI & Importância & $3,129 \times 10^{-5}$ & 0,0223 & 1,20 \\
\hline $\mathbf{A A}$ & Assintótica & $8,067 \times 10^{-5}$ & 4,1835 & 154,71 \\
\hline $\mathbf{A M}$ & Melhorada & $1,102 \times 10^{-4}$ & 0,6168 & 247,95 \\
\hline SS & Subconjuntos & $4,881 \times 10^{-5}$ & 0,2247 & 54,12 \\
\hline
\end{tabular}

O resultado apresentado na Tabela 8 é ilustrado graficamente na Figura 36. Neste caso, se observa que o Monte Carlo Bruto não apresenta resultados para uma amostra deste tamanho. Por outro lado, o Monte Carlo com Amostragem por Importância apresenta uma vantagem significativa em relação às outras técnicas, como era esperado. É observado que a técnica de Simulação de Subconjuntos apresenta um bom resultado em termos do valor médio e do coeficiente de variação da probabilidade de falha. Destaca-se que o uso da Amostragem por Hipercubo Latino é benéfico para a maioria das técnicas, uma vez que ela leva a um 
resultado mais próximo da probabilidade de falha de referência, tal como observado pelo valor do erro relativo.

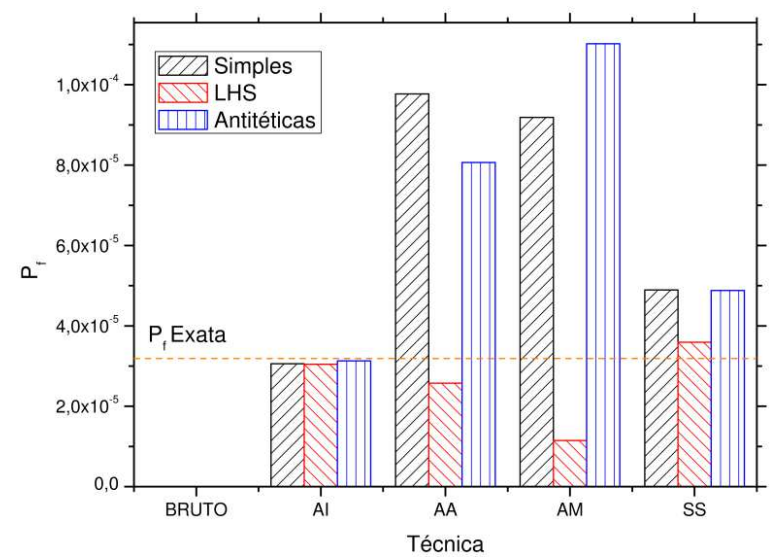

Figura 36. Comparativo da probabilidade de falha para uma amostra de tamanho 9.200, no Exemplo 1.

\subsubsection{Tempo de processamento}

Nesta seção são apresentados os gráficos da relação entre o tempo de processamento e o tamanho da amostra. Na Figura 37 e na Figura 38, comparam-se os tempos de processamento do método de Monte Carlo Bruto (BRUTO), do método de Monte Carlo com Amostragem por Importância (AI), da Amostragem Assintótica (AA) e da Amostragem Melhorada (AM). Utilizam-se a Amostragem Simples, a Amostragem por Hipercubo Latino e a Amostragem por Variáveis Antitéticas, como técnicas de amostragem básica. O tamanho da amostra varia de $1 \times 10^{5}$ até $9 \times 10^{5}$. Já na Figura 39a comparam-se os tempos de processamento da técnica de Simulação de Subconjuntos (SS) e na Figura 39b comparam-se os tempos de processamento de todas as técnicas estudadas neste trabalho. Na Figura 39a e na Figura 39b, o tamanho da amostra varia de 6.900 até 43.700. Para a Simulação de Subconjuntos, consideram-se os mesmos parâmetros utilizados no estudo comparativo, com o tamanho de cada subconjunto variando de 1.500 até 9.500 de 2.000 em 2.000. 
a) Monte Carlo Bruto

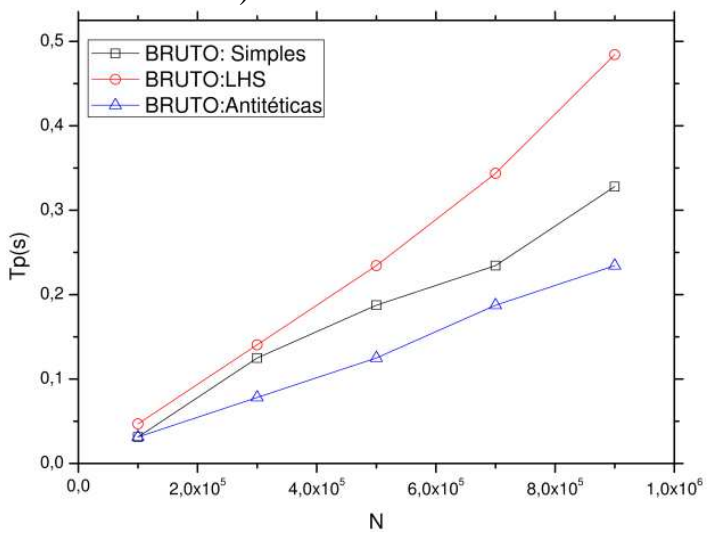

b) Amostragem por Importância

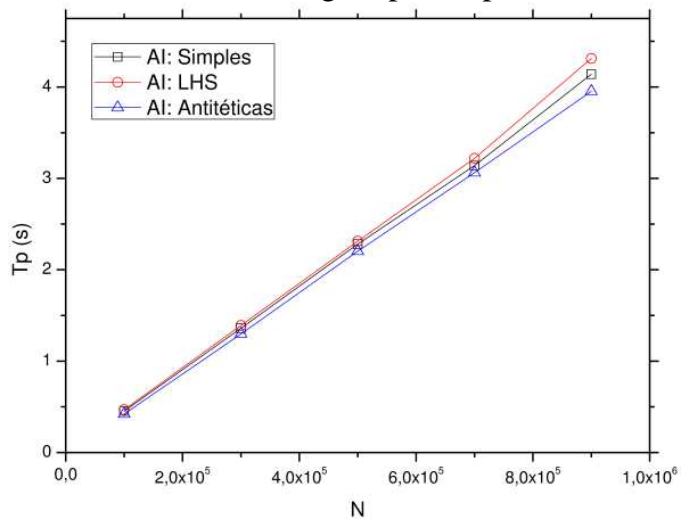

Figura 37. Relação entre o tempo de processamento e o tamanho da amostra no Exemplo 1: Monte Carlo Bruto (a) e Amostragem por Importância (b).

a) Amostragem Assintótica

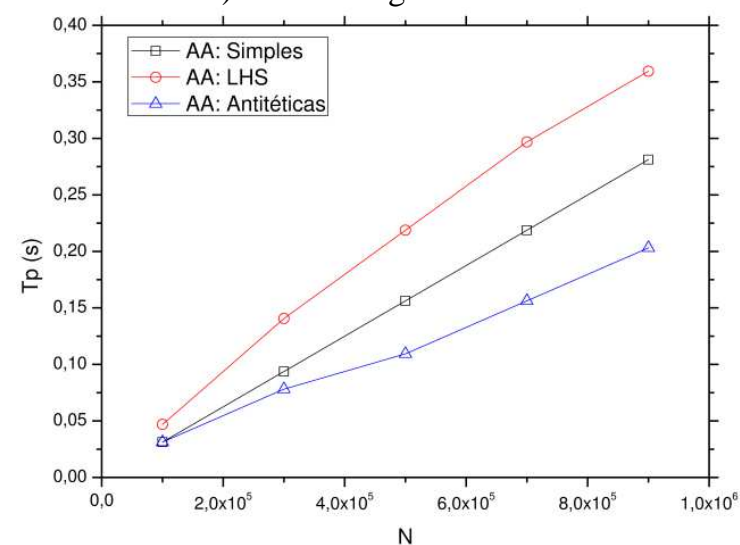

b) Amostragem Melhorada

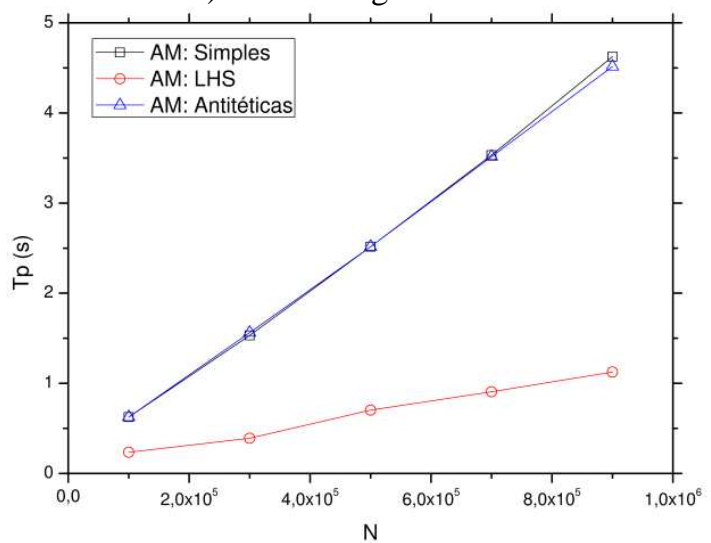

Figura 38. Relação entre o tempo de processamento e o tamanho da amostra no Exemplo 1: Amostragem Assintótica (a) e Amostragem Melhorada (b).

a) Simulação de Subconjunto

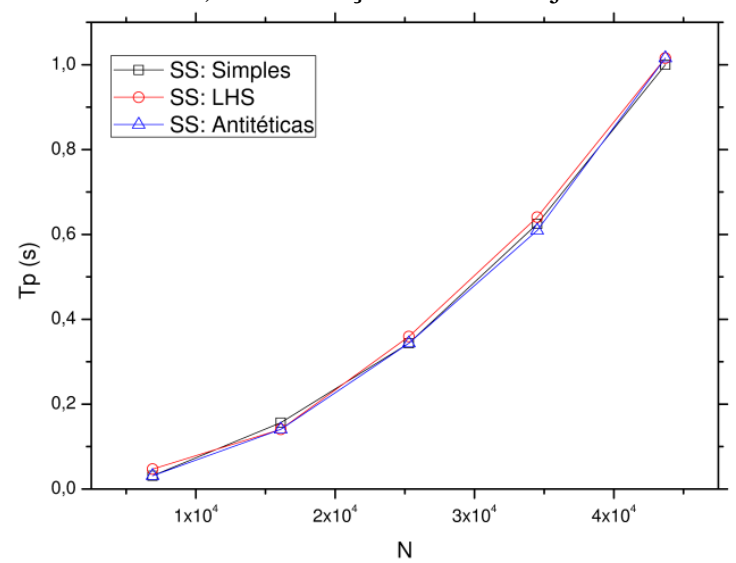

b) Todas as técnicas

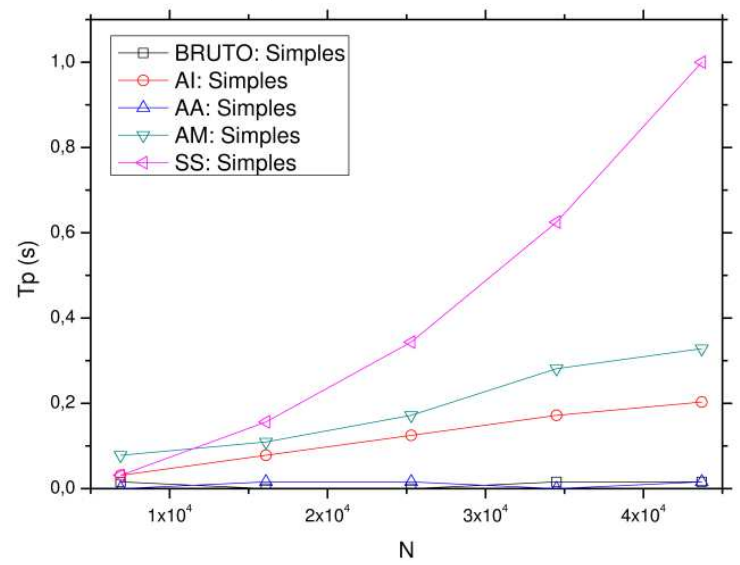

Figura 39. Relação entre o tempo de processamento e o tamanho da amostra no Exemplo 1: Simulação de Subconjuntos (a) e comparativo entre todas as técnicas(b).

Na Figura 37a, na Figura 37b e na Figura 38a, observa-se que o uso da Amostragem por Hipercubo Latino elevou os tempos de processamento da simulação do Monte Carlo Bruto, da 
Amostragem por Importância e da Amostragem Assintótica. Por outro lado, a técnica de Amostragem por Variáveis Antitéticas apresentou um tempo de processamento menor. Esse comportamento é decorrente da maneira em que são geradas as amostras na Amostragem por Hipercubo Latino e por Variáveis Antitéticas. Destaca-se que, devido ao fato de a Amostragem por Importância apresentar um custo computacional elevado, as técnicas de amostragem básica apresentam uma menor influência no tempo de processamento.

Na Figura 38b, observa-se que a Amostragem Melhorada utilizando a Amostragem por Hipercubo Latino, apresentou um custo computacional menor. Porém, deve-se destacar que a Amostragem por Hipercubo Latino apresenta um maior consumo de memória.

Na Figura 39a, observa-se que o uso de técnicas de amostragem básica na Simulação de Subconjuntos, não exercem forte influência no tempo de processamento, porém, o comportamento da curva que relaciona o tamanho da amostra com o tempo de processamento é não linear. Isso poderia ser considerado um problema, caso a técnica de Simulação de Subconjuntos necessitasse realizar um número grande de simulações, mas esse não é o caso, e essa é a principal vantagem da técnica. Na Figura 39b é possível comparar todas as técnicas, utilizando a Amostragem Simples e variando o tamanho da amostra de 6.900 até 43.700. Neste caso, nota-se que o tempo de processamento da Simulação de Subconjuntos é maior que o das outras técnicas. Porém, com uma amostra pequena, a técnica de Simulação de Subconjuntos já apresenta bons resultados, o que pode ser útil em problemas onde a equação de estado limite utiliza métodos numéricos de alto custo computacional (e.g. Elementos Finitos considerando não linearidades).

\subsection{Exemplo 2: Variáveis Aleatórias Limitadas}

Este exemplo analisa a aplicação das técnicas estudadas em um problema com variáveis aleatórias limitadas nas caudas. O problema a ser analisado é do tipo Capacidade-Demanda, sendo que neste exemplo são adotadas variáveis aleatórias com distribuição uniforme de probabilidade. A equação de estado limite deste problema é dada por:

$$
g(\mathrm{C}, \mathrm{D})=\mathrm{C}-\mathrm{D}=0
$$

Os parâmetros das distribuições de probabilidade de cada variável aleatória são apresentados na Tabela 9. São consideradas variáveis aleatórias independentes. 
Tabela 9. Parâmetros das variáveis aleatórias do Exemplo 2.

\begin{tabular}{clll}
\hline Variável Aleatória & Distribuição & A & B \\
\hline C & Uniforme & 10,0 & 30,0 \\
D & Uniforme & 1,1 & 10,1 \\
\hline
\end{tabular}

Este problema pode ser resolvido analiticamente, uma vez que a probabilidade de falha é definida por:

$$
P_{f}=\int_{-\infty}^{\infty} f_{D}(\delta) F_{C}(\delta) d \delta
$$

Dessa forma, a probabilidade de falha vem a ser a área sob a curva $f_{D}(\delta) F_{C}(\delta)$, tal como visto na Figura 40.

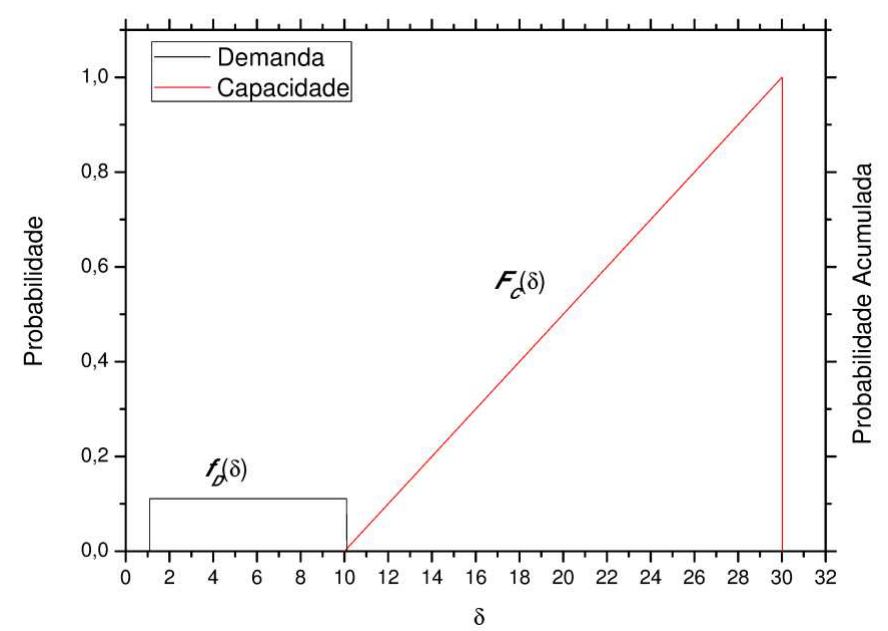

Figura 40. FDP da variável Demanda (D) e FDA da variável Capacidade (C), para o Exemplo 2.

Neste caso, tem-se que:

$$
\begin{aligned}
& f_{D}(\delta)=0,1111111 \text { para } \delta \text { entre } 1,1 \text { e } 10,1 \\
& F_{C}(\delta)=0,05(\delta-10) \text { para } \delta \text { entre } 10 \text { e } 30 .
\end{aligned}
$$

O produto destas duas funções resulta na função apresentada na Figura 41, onde se está interessado na área abaixo dela (área hachurada). 


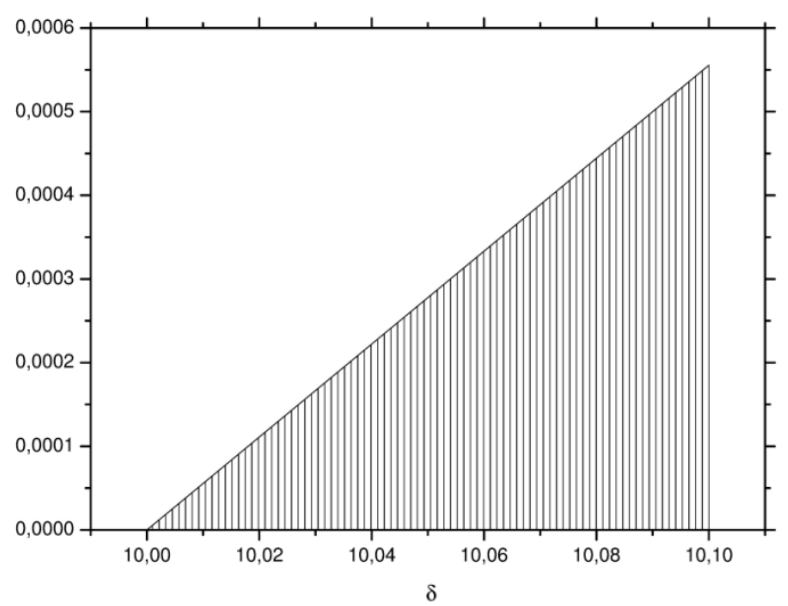

Figura 41. Curva a ser integrada no cálculo da probabilidade de falha, no Exemplo 2.

Então se pode escrever a probabilidade de falha como:

$$
P_{f}=\int_{-\infty}^{\infty} 0,1111111 \cdot 0,05(\delta-10) d \delta=2,778 \times 10^{-5}
$$

Com este resultado se obtém um índice de confiabilidade $\beta=4,0309$.

O FORM não apresentou convergência neste problema, o que interfere diretamente na Amostragem por Importância, pois, a técnica que está implementada no StRAnD é a Amostragem por Importância usando os pontos de projeto, e como este não pôde ser definido, a técnica não pode ser utilizada de maneira apropriada.

Seguindo o procedimento adotado neste trabalho, são apresentados resultados para a técnica de Amostragem Assintótica, seguida pela Amostragem Melhorada e pela técnica de Simulação de Subconjuntos. Por fim, é apresentado um estudo comparativo entre as técnicas estudadas.

\subsubsection{Amostragem Assintótica}

É realizada a análise do comportamento do índice de confiabilidade $\beta$ com o parâmetro $f$. Neste caso, as variáveis podem assumir valores negativos. Adota-se o intervalo de $f$ entre 0,3 e 1,0 , sendo que $f=1,0$ representa o problema original. Gerou-se 20 pontos de suporte dentro deste intervalo. Para cada ponto de suporte foram realizadas $2 \times 10^{6}$ simulações de Monte Carlo. A técnica de Amostragem Assintótica faz uso da Eq. (108) e da Eq. (109) no ajuste não linear. É utilizada apenas a Amostragem Simples em cada ponto de suporte, uma vez que a Amostragem por Hipercubo Latino e a Amostragem por Variáveis Antitéticas 
apresentaram resultados muito similares. Assim, é possível obter os gráficos apresentados na Figura 42.

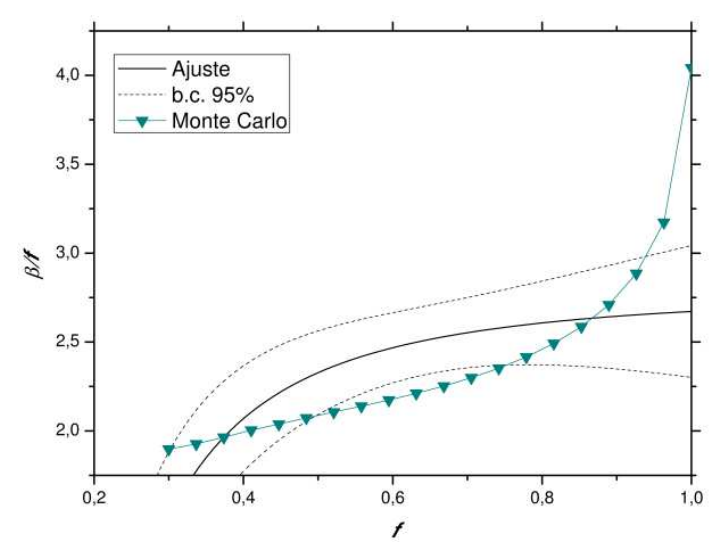

a) Para $C=-2$ na Eq. (109).

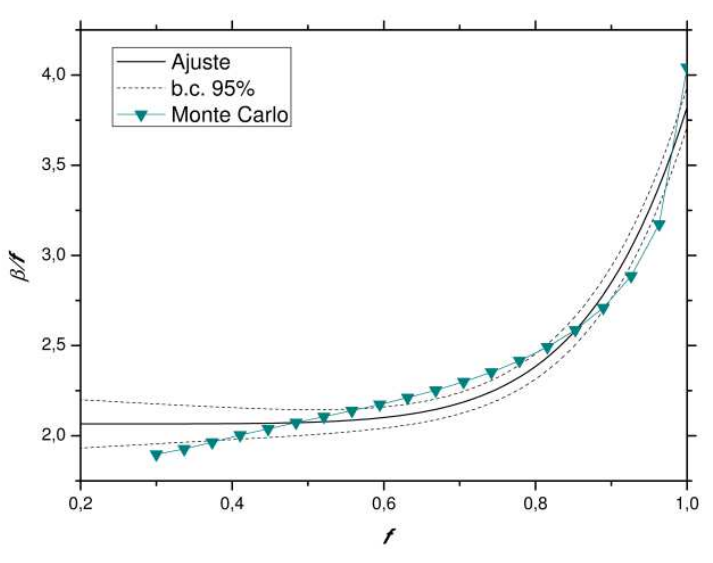

b) Para $C$ livre na Eq. (109).

Figura 42. Regressão não linear na Amostragem Assintótica, para o Exemplo 2.

Observa-se na Figura 42, seja para o caso particular com $C=-2$ ou para $C$ livre, que não há uma boa concordância da função a ser ajustada, com as duplas $(f, \beta / f)$. Esse comportamento pode ser explicado pela forma da distribuição conjunta das variáveis aleatórias, tal como observado na Figura 43.

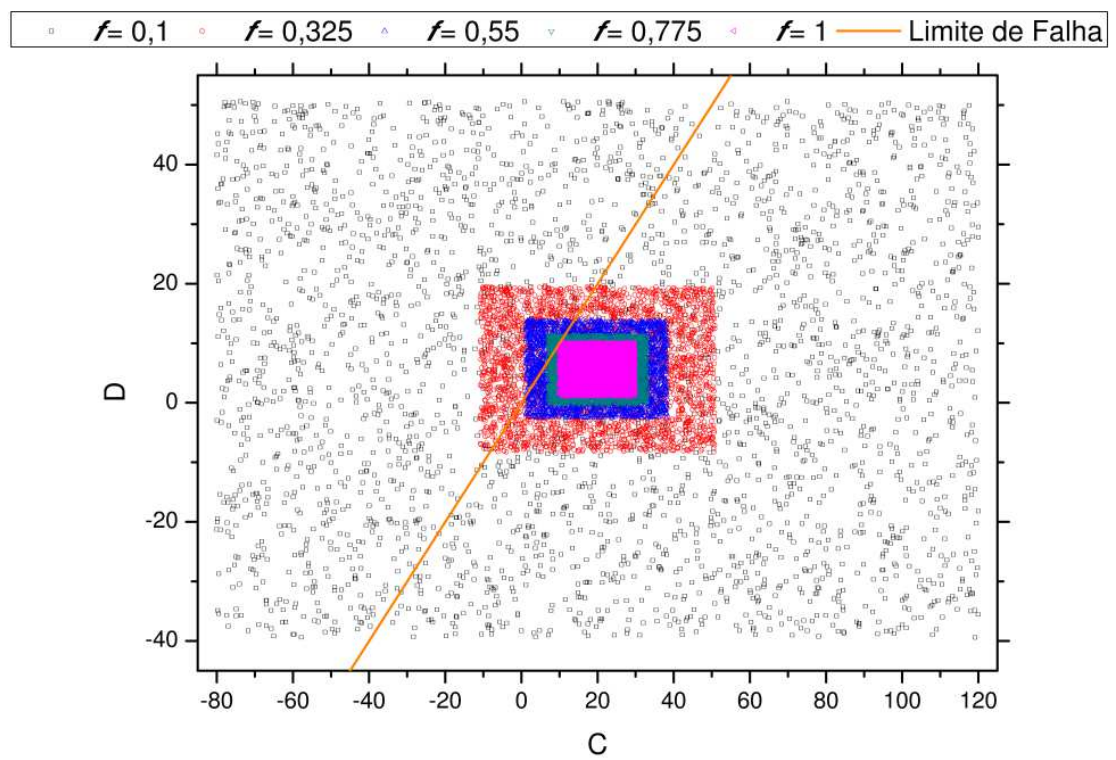

Figura 43. Espaço amostral parametrizado utilizando a Amostragem Assintótica, no Exemplo 2.

Uma vez que os desvios-padrão das variáveis aleatórias aumentam, os limites inferior e superior de cada distribuição aumentam de acordo com $f$. Dessa forma, para cada valor de $f$, um novo espaço amostral é obtido. Assim, diferentes domínios de falha são obtidos, e a 
probabilidade de falha é proporcional à área destes domínios de falha. Através de uma análise geométrica pode-se obter o gráfico da Figura 44, que é a relação entre o índice de confiabilidade parametrizado por $f$, obtido pela probabilidade de falha relativa às áreas dos domínios de falha parametrizados, com o valor do parâmetro $f$. Esse resultado concorda com aquele obtido pela simulação de Monte Carlo, tal como apresentados na Figura 42.

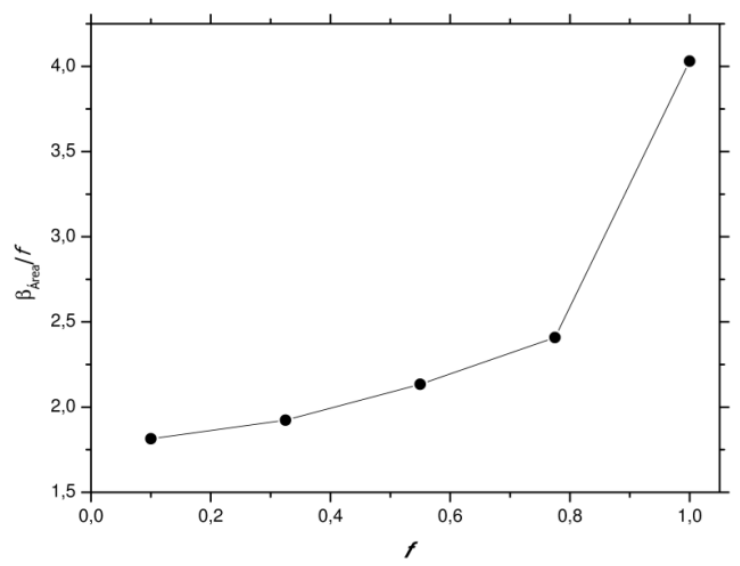

Figura 44. Relação entre o índice de confiabilidade, relativo à area do domínio de falha, e o parâmetro $f$, na Amostragem Assintótica, para Exemplo 2.

\subsubsection{Amostragem Melhorada}

Procedimento idêntico ao adotado na Amostragem Assintótica é realizado para o estudo da Amostragem Melhorada. Inicialmente é analisada a concordância da equação proposta por Naess, Leira e Batsevych (2009), com as duplas $\left(\lambda, P_{f}\right)$. São adotados 100 pontos de suporte, com $\lambda$ variando de 0,4 até 1,0 . Considera-se uma amostra de tamanho $1 \times 10^{7}$. Utiliza-se a Amostragem Simples como amostragem básica. Tal resultado pode ser observado na Figura 45, onde se nota que não há uma boa concordância da curva proposta com os pontos de suporte, o que ocasiona erros na estimativa da probabilidade de falha quando $\lambda=1,0$. 


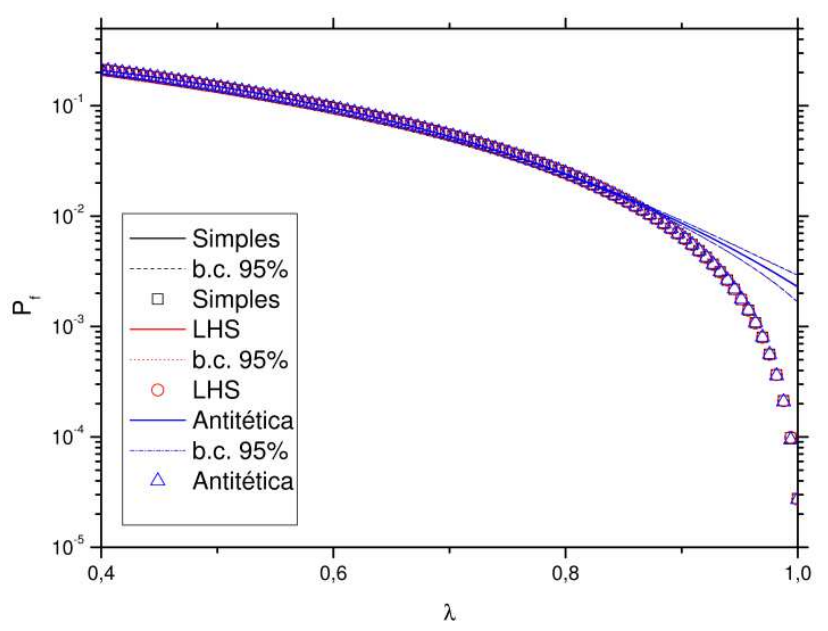

Figura 45. Regressão não linear na Amostragem Melhorada, para o Exemplo 2.

Este comportamento dos pontos de suporte está diretamente relacionado com a forma da distribuição conjunta de probabilidade, tal como na Amostragem Assintótica. Uma vez que a equação de estado limite é deslocada para a origem do espaço de projeto pelo parâmetro $\lambda$, diferentes domínios de falha vão sendo obtidos (Figura 46). Assim, para cada domínio de falha são feitas estimativas da probabilidade de falha parametrizada. Dessa forma, a geometria do domínio de falha governa o comportamento da probabilidade de falha.

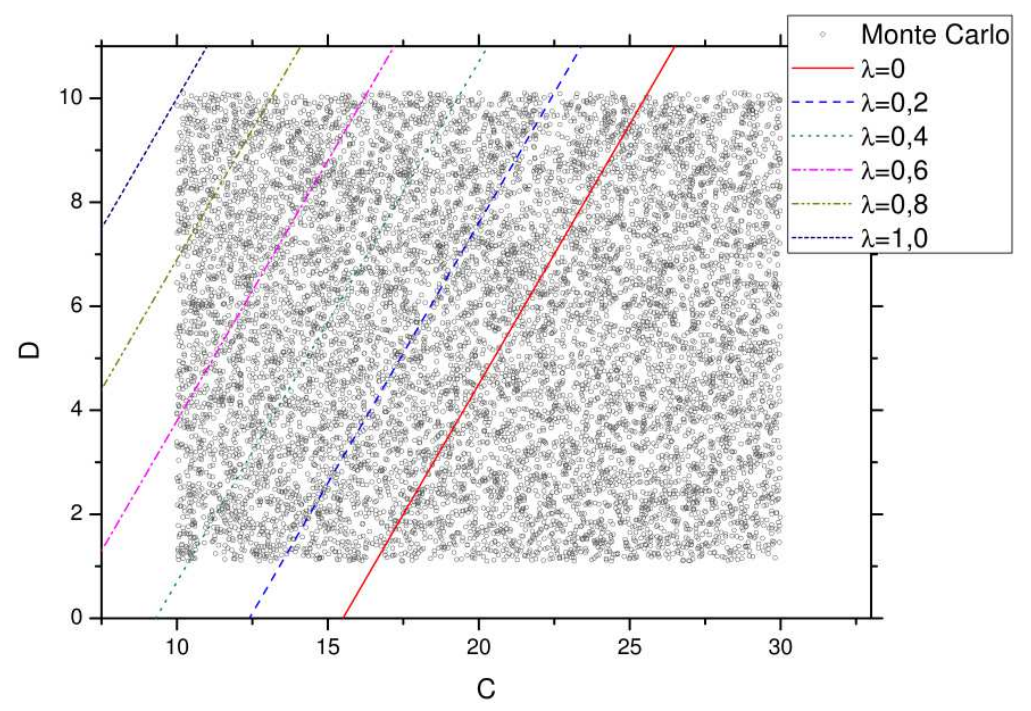

Figura 46. Limite de falha parametrizado, para o Exemplo 2.

Na Figura 46, cada linha é definida por um valor de $\lambda$, que por sua vez define um limite de falha parametrizado. Sendo assim, a probabilidade de falha é proporcional à área definida por este limite de falha parametrizado. Geometricamente tais áreas podem ser facilmente calculadas, tornando possível o cálculo das áreas relativas de cada domínio de falha. Na Figura 47 é possível observar que o resultado da análise geométrica é equivalente ao gráfico 
apresentado na Figura 45. Assim, as técnicas de Amostragem Assintótica e Amostragem Melhorada, não são adequadas para resolver este problema. Desta forma, observa-se que tais técnicas apresentam uma dependência do problema que se queira analisar. Então, é fundamental conhecer as características das variáveis aleatórias de cada problema.

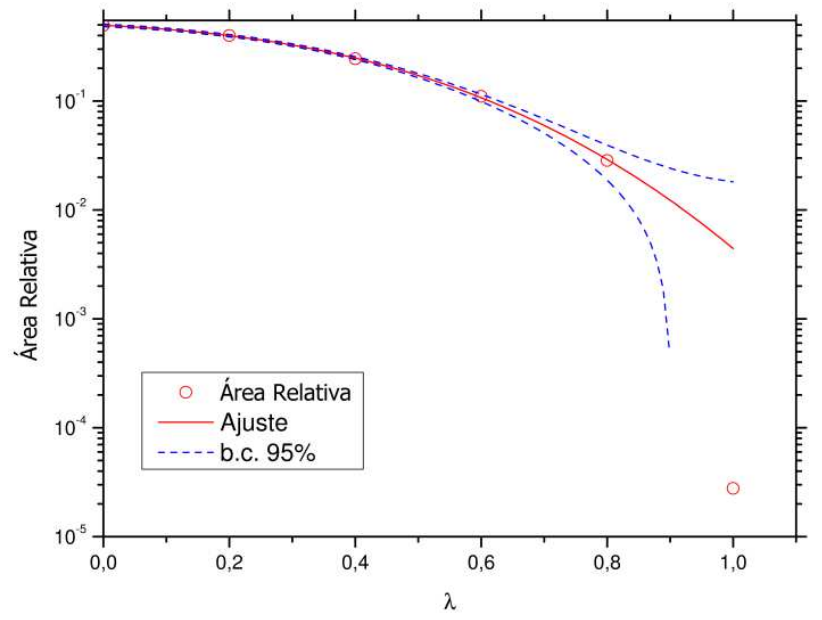

Figura 47. Área relativa do domínio de falha parametrizado, para o Exemplo 2.

\subsubsection{Simulação de Subconjuntos}

Os dados de entrada do StRAnD, que definem o caminhante aleatório utilizado na Simulação de Subconjuntos, são apresentados na Tabela 10.

Tabela 10. Médias e desvios padrão do caminhante aleatório do Exemplo 2.

\begin{tabular}{clll}
\hline Variável Aleatória & Distribuição & Média & Desvio padrão \\
\hline C & Uniforme & 0,0 & $\alpha \times 5,7735$ \\
D & Uniforme & 0,0 & $\alpha \times 2,5981$ \\
\hline
\end{tabular}

onde $\alpha$ é uma constante multiplicativa maior que zero, que irá alterar o valor do desvio-padrão do caminhante aleatório.

Utilizando um caminhante aleatório uniforme $\operatorname{com} \alpha=0,2, P_{0}=0,1$ e uma amostra com 800 pontos em cada subconjunto, é possível obter o gráfico apresentado na Figura 48, onde é ilustrada a formação de cinco subconjuntos. Dessa forma, é possível observar que os subconjuntos gerados apresentam um bom comportamento para o problema em análise. 


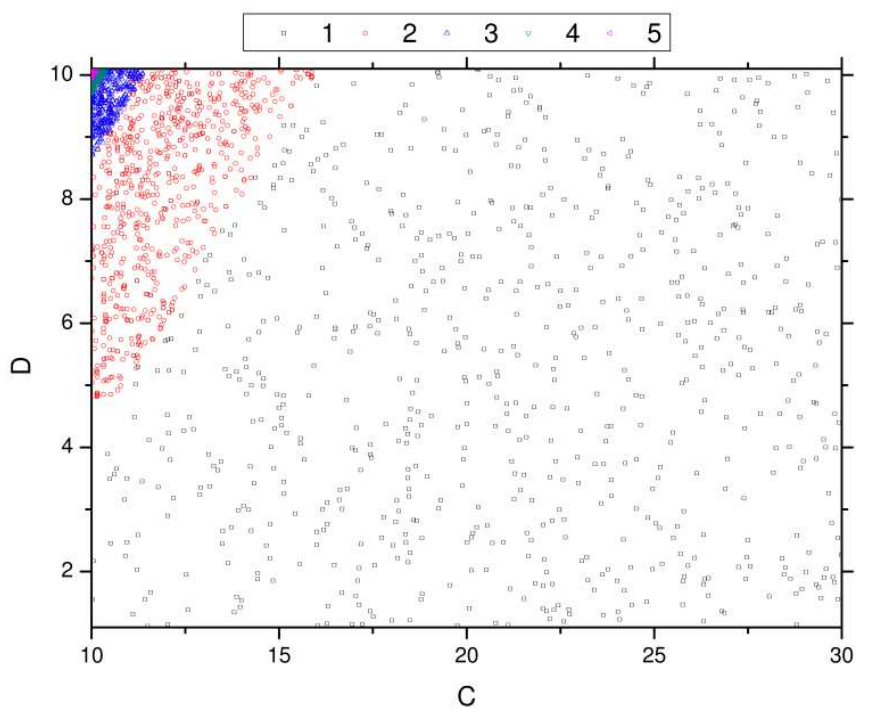

Figura 48. Subconjuntos gerados via cadeias de Markov por meio do algoritmo de Metropolis-Hastings Modificado.

\subsubsection{Análise de convergência}

Nesta seção é realizado o estudo da convergência da probabilidade de falha em relação ao tamanho da amostra. São analisadas as técnicas que apresentaram resultados satisfatórios. Portanto, é realizado o estudo da técnica de simulação de Monte Carlo Bruto, com o tamanho da amostra variando de $1 \times 10^{5}$ a $1 \times 10^{8}$. Assim, é possível obter os gráficos de convergência apresentados na Figura 49, na Figura 50 e Figura 51.

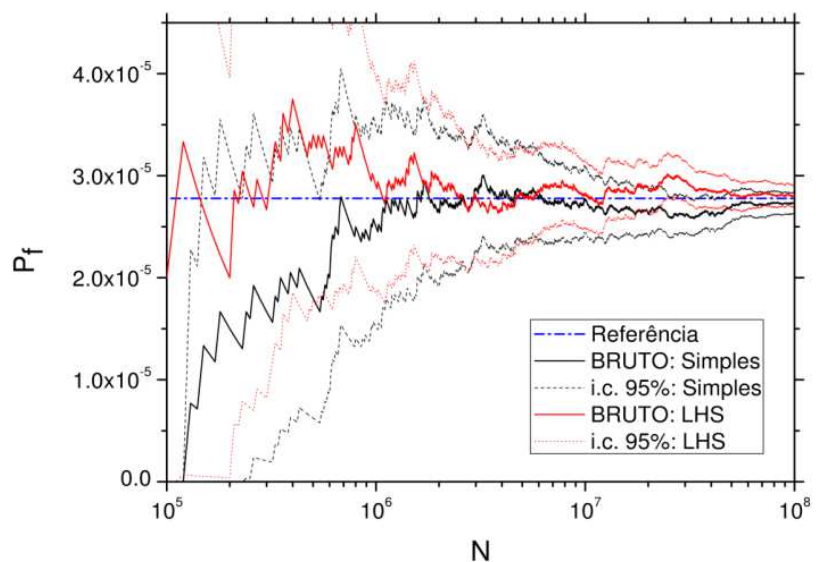

Figura 49. Comparativo da convergência de $P_{f}$ para o Monte Carlo Bruto com Amostragem Simples e com Amostragem por Hipercubo Latino, para o Exemplo 2. 


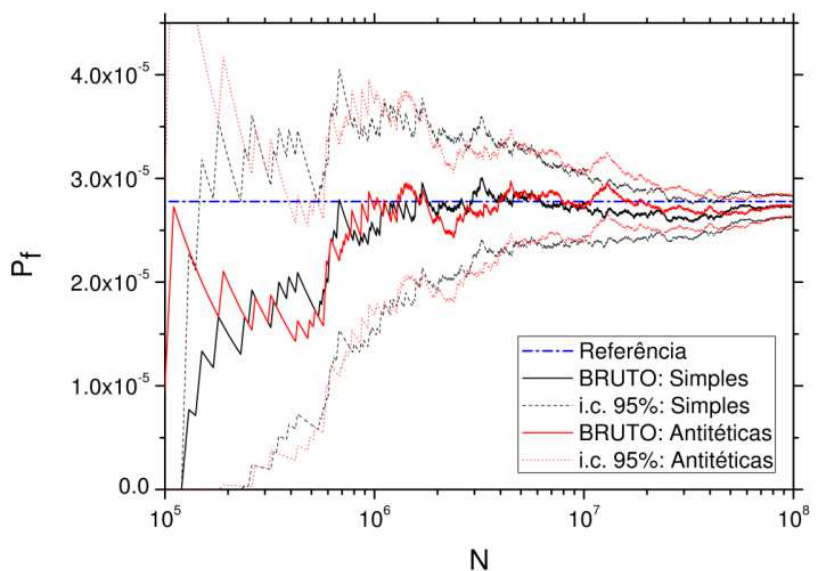

Figura 50. Comparativo da convergência de $P_{f}$ para o Monte Carlo Bruto com Amostragem Simples e com Amostragem por Variáveis Antitéticas, para o Exemplo 2.

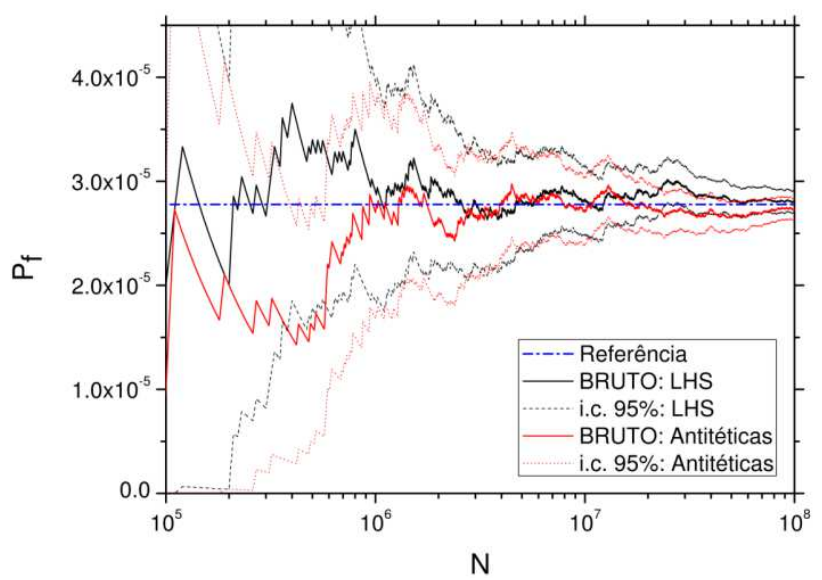

Figura 51. Comparativo da convergência de $P_{f}$ para o Monte Carlo Bruto com Amostragem por Hipercubo Latino e com Amostragem por Variáveis Antitéticas, para o Exemplo 2.

Observa-se que há uma leve melhora, em relação à convergência da probabilidade de falha, quando se utiliza a Amostragem por Hipercubo Latino, na simulação de Monte Carlo com Amostragem Bruta.

Na Figura 52, observa-se que nas primeiras simulações o coeficiente de variação da probabilidade de falha é um pouco menor quando se utiliza a Amostragem por Hipercubo Latino e por a Amostragem por Variáveis Antitéticas, porém, essa diferença tende a diminuir à medida que se aumenta o tamanho da amostra. 


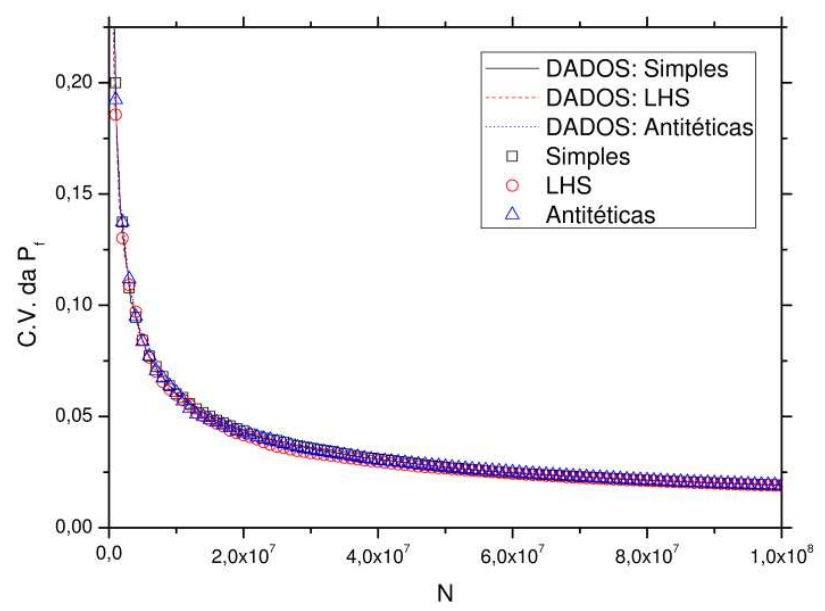

Figura 52. Coeficiente de variação da probabilidade de falha na Amostragem por Importância, para o Exemplo 2.

\subsubsection{Comparativo da probabilidade de falha e de seu coeficiente de variação}

É realizado um comparativo entre o Monte Carlo Bruto e a Simulação de Subconjuntos. O tamanho da amostra é fixado em 3.680, onde na técnica de Simulação de Subconjuntos se têm 800 pontos amostrais em cada subconjunto. Tal resultado é expresso na Tabela $11 \mathrm{e}$ ilustrado na Figura 53. Foram adotados os seguintes parâmetros: $\alpha=0,2$ e $P_{0}=0,1$.

Tabela 11. Comparativo da $P_{f}$ para uma amostra de tamanho 3.680, no Exemplo 2.

\begin{tabular}{|c|c|c|c|c|}
\hline \multicolumn{5}{|c|}{$P_{f}$ de referência: $2,778 \times 10^{-5}$} \\
\hline \multicolumn{5}{|c|}{ SIMPLES } \\
\hline Sigla & Técnica & $\boldsymbol{P}_{f}$ & C.V.da $P_{f}$ & Erro (\%) \\
\hline BRUTO & Bruto & -- & -- & -- \\
\hline SS & Subconjuntos & $2,388 \times 10^{-5}$ & 0,4271 & 14,0388 \\
\hline \multicolumn{5}{|c|}{ HIPERCUBO LATINO } \\
\hline Sigla & Técnica & $\boldsymbol{P}_{f}$ & C.V.da $P_{f}$ & \\
\hline BRUTO & Bruto & -- & -- & -- \\
\hline SS & Subconjuntos & $2,430 \times 10^{-5}$ & 0,4191 & 12,5270 \\
\hline \multicolumn{5}{|c|}{ VARIÁVEIS ANTITÉTICAS } \\
\hline Sigla & Técnica & $\boldsymbol{P}_{f}$ & C.V.da $P_{f}$ & \\
\hline BRUTO & Bruto & - & -- & -- \\
\hline SS & Subconjuntos & $1,559 \times 10^{-5}$ & 0,4216 & 43,8805 \\
\hline
\end{tabular}

Na Tabela 11 e na Figura 53, se observa que a técnica de Simulação de Subconjunto, com uma amostra pequena, obteve uma boa estimativa da probabilidade de falha. Por outro lado, o Monte Carlo Bruto não apresenta resultados de probabilidade de falha para uma amostra desse tamanho. Assim, a Simulação de Subconjuntos pode ser útil quando se deseja 
avaliar a probabilidade de falha em problemas onde as demais técnicas falham ou não são computacionalmente viáveis.

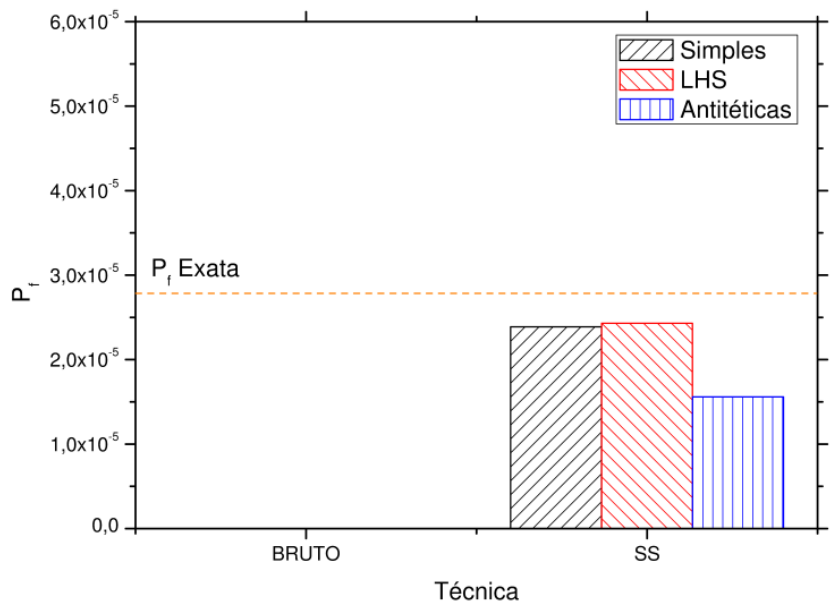

Figura 53. Comparativo da probabilidade de falha para uma amostra de tamanho 3.680, no Exemplo 2.

\subsubsection{Tempo de processamento}

As únicas técnicas que são comparadas nesta análise são a simulação de Monte Carlo Bruto e a Simulação de Subconjuntos, ambas utilizando a Amostragem Simples, a Amostragem por Hipercubo Latino e a Amostragem por Variáveis Antitéticas. O tamanho da amostra é variado de 6.900 até 43.700. Para a Simulação de Subconjuntos, consideram-se os mesmos parâmetros utilizados no estudo comparativo realizado em 7.2.5. O tamanho da amostra em cada subconjunto varia de 1.500 até 9.500, de 2.000 em 2.000. Já para o Monte Carlo Bruto (Figura 54b), o tamanho da amostra varia de $1 \times 10^{5}$ até $9 \times 10^{5}$.

Na Figura 54a e na Figura 54b, observa-se que o uso da Amostragem por Hipercubo Latino e da Amostragem por Variáveis Antitéticas não traz nenhum prejuízo ao Monte Carlo Bruto, nem para a Simulação de Subconjuntos. Na Figura 54a, se observa que a técnica de Simulação de Subconjuntos apresenta um maior tempo de processamento, entretanto sabe-se que esta técnica apresenta melhores estimativas da probabilidade de falha para uma amostra pequena. 


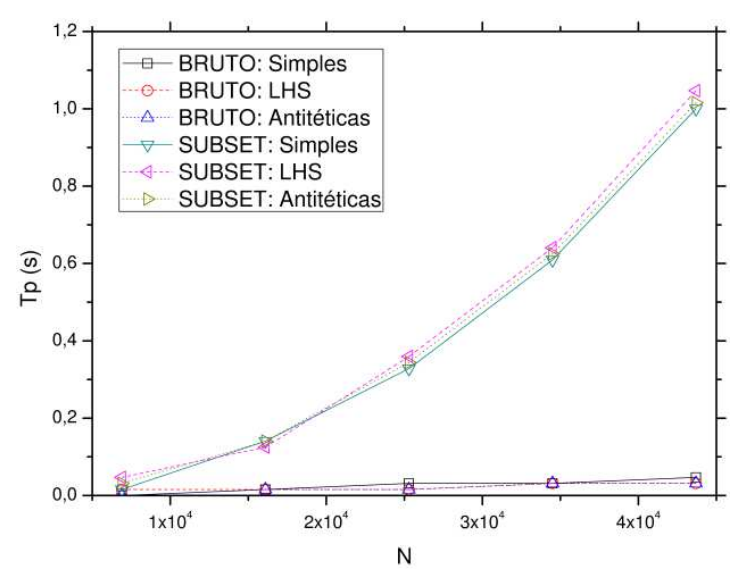

a)

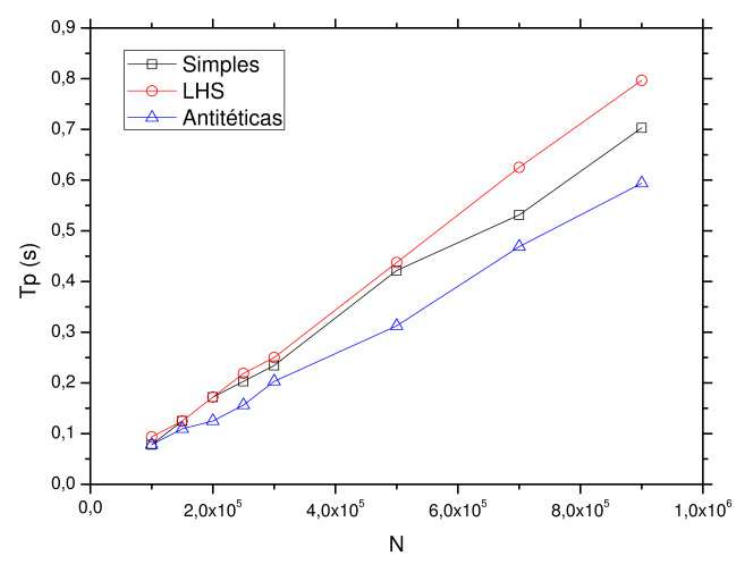

b)

Figura 54. Relação entre o tempo de processamento e o tamanho da amostra no Exemplo 2: comparativo entre todas as técnicas (a) e Monte Carlo Bruto (b).

\subsection{Exemplo 3: Equação de estado limite não linear}

Este exemplo estuda a aplicação das técnicas apresentadas, na resolução de um problema com uma equação de estado limite não linear. $\mathrm{O}$ estado limite é definido pela seguinte função:

$$
g\left(\mathrm{X}_{1}, \mathrm{X}_{2}\right)=3-\mathrm{X}_{2}+\left(4 \mathrm{X}_{1}\right)^{4}
$$

Esta função é fortemente não linear e seu comportamento pode ser visto na Figura 55.

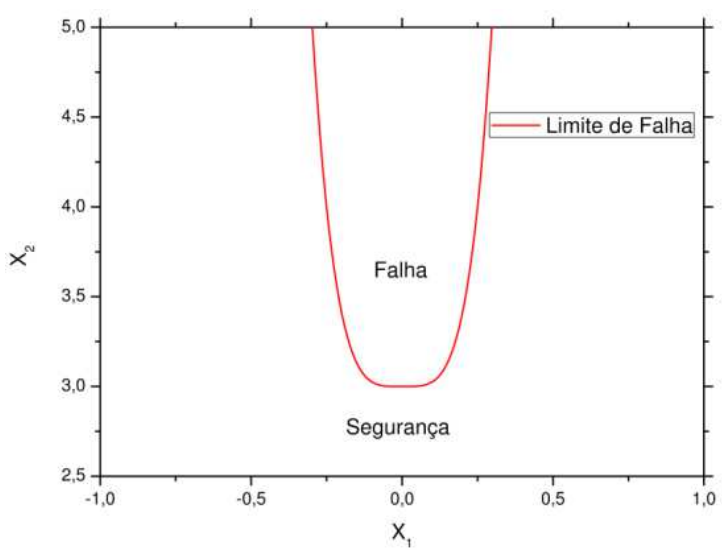

Figura 55. Limite de falha e os domínios de falha e segurança, para o Exemplo 3.

As distribuições e propriedades de cada variável aleatória são apresentadas na Tabela 12. 
Tabela 12. Médias e desvios padrão das variáveis aleatórias do Exemplo 3.

\begin{tabular}{clll}
\hline Variável Aleatória & Distribuição & Média & Desvio Padrão \\
\hline $\mathbf{X}_{\mathbf{1}}$ & Normal & 0,00 & 1,00 \\
$\mathbf{X}_{\mathbf{2}}$ & Normal & 0,00 & 1,00 \\
\hline
\end{tabular}

Para obter a probabilidade de falha de referência desse problema é utilizado o Monte Carlo Bruto com Amostragem Simples com uma amostra de tamanho $9 \times 10^{8}$. A probabilidade de falha encontrada é de $1,774 \times 10^{-4}(\beta=3,5716)$, com coeficiente de variação de 0,0025 . Tal simulação durou 357,28s ( 6 min). É utilizado o FORM de forma a indicar o erro que este comete devido ao procedimento de linearização da função de estado limite. De acordo com o FORM, o ponto de projeto encontrado é $x^{*}=(0,0 ; 3,0)$, o que leva a uma probabilidade de falha de $1,35 \times 10^{-3}$ e a um índice de confiabilidade $\beta=3,0$. Assim, pode-se afirmar que o FORM não apresenta uma boa estimativa da probabilidade de falha para uma equação de estado limite é fortemente não linear.

\subsubsection{Amostragem Assintótica}

Nesta seção, é realizado um estudo da aplicação da técnica de Amostragem Assintótica. Nos gráficos de regressão são variados o tamanho da amostra e as técnicas de amostragem básica (Amostragem Simples, Amostragem por Hipercubo Latino e Amostragem por Variáveis Antitética). Tal resultado pode ser visto na Figura 56 e na Figura 57. São utilizados 5 pontos de suporte, sendo os tamanho das amostras de $3 \times 10^{3}$ (Figura 56) e de $1 \times 10^{5}$ (Figura 57), $\operatorname{com} f$ variando de 0,3 a 0,7 . Esses valores são escolhidos por apresentarem um resultado significativo para o estudo, em termos de qualidade do ajuste.

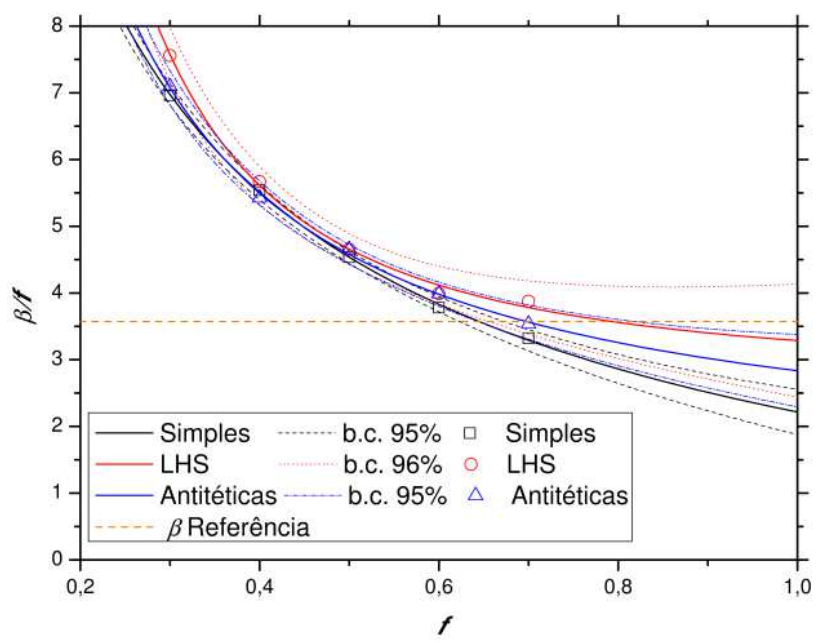

Figura 56. Regressão não linear utilizada na Amostragem Assintótica para amostras de tamanho $3 \times 10^{3}$, no Exemplo 3. 


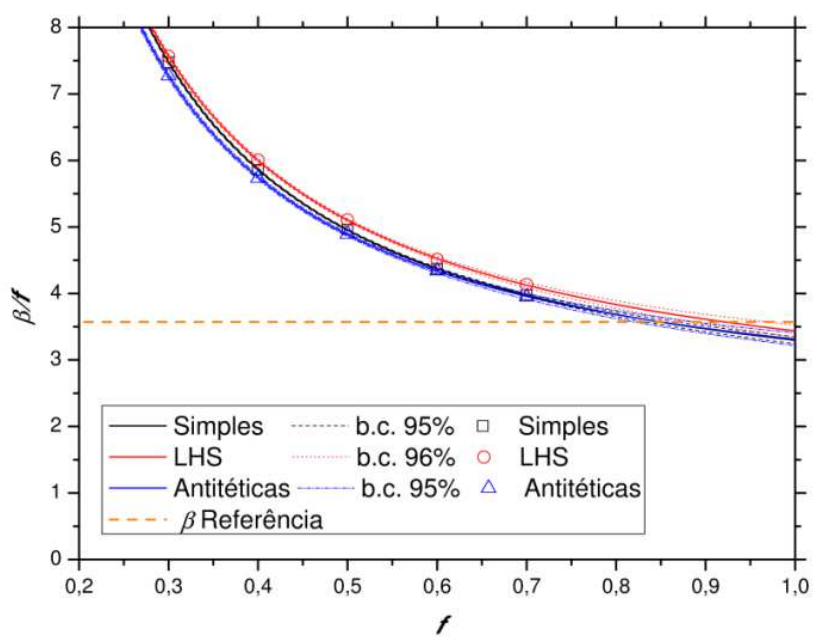

Figura 57. Regressão não linear utilizada na Amostragem Assintótica para amostras de tamanho $1 \times 10^{5}$, no Exemplo 3.

Os parâmetros das funções ajustadas para a técnica em questão, com a Amostragem Simples (ASMC), com a Amostragem por Hipercubo Latino (ALHS) e com a Amostragem com Variáveis Antitéticas (AASMC), são apresentados na Tabela 13, para uma amostra de tamanho $3 \times 10^{3}$ e outra de tamanho $1 \times 10^{5}$. Assim, se observa que o valor do parâmetro $C$ não é mais igual a -2, como descrito na Eq. (108).

Tabela 13. Parâmetros ajustados na Amostragem Assintótica, para o Exemplo 3.

\begin{tabular}{ccccccc}
\hline $\boldsymbol{N}$ & \multicolumn{3}{c}{$\mathbf{3 \times 1 0}^{\mathbf{3}}$} & \multicolumn{3}{c}{$\mathbf{1 \times 1 0}^{\mathbf{5}}$} \\
\hline & $\boldsymbol{A}$ & $\boldsymbol{B}$ & $\boldsymbol{C}$ & $\boldsymbol{A}$ & $\boldsymbol{B}$ & $\boldsymbol{C}$ \\
ASMC & 2,3286 & 4,5452 & $-0,5949$ & 2,0617 & 1,2357 & $-1,2270$ \\
ALHS & 2,7393 & 0,5476 & $-1,8091$ & 2,1243 & 1,3134 & $-1,1811$ \\
AASMC & 1,3554 & 1,4792 & $-1,1235$ & 2,1389 & 1,1745 & $-1,2248$ \\
\hline
\end{tabular}

$\mathrm{Na}$

Tabela 14, pode-se observar o comparativo da resposta da técnica de Amostragem Assintótica para diferentes tamanho de amostra, com a Amostragem Simples, com a Amostragem por Hipercubo Latino e com a Amostragem por Variáveis Antitéticas. Nota-se que o uso da Amostragem por Hipercubo Latino leva a um valor de probabilidade de falha mais proximo do valor de referência, seja para uma amostra de tamanho $3 \times 10^{3}$ ou de $1 \times 10^{5}$.

Tabela 14. Probabilidade de falha e coeficiente de Variação da probabilidade de falha, para a Amostragem Assintótica, no Exemplo 3.

\begin{tabular}{|c|c|c|c|c|}
\hline \multicolumn{5}{|c|}{$P_{f}$ de referência: $1,774 \times 10^{-4}$} \\
\hline$N$ & \multicolumn{2}{|c|}{$3 \times 10^{3}$} & \multicolumn{2}{|c|}{$1 \times 10^{5}$} \\
\hline & $P_{f}$ & C.V. & $P_{f}$ & C.V. \\
\hline ASMC & $1,333 \times 10^{-2}$ & 0,6486 & $4,88 \times 10^{-4}$ & 0,1026 \\
\hline ALHS & $5,065 \times 10^{-4}$ & 0,0915 & $2,93 \times 10^{-4}$ & 0,1994 \\
\hline AASMC & $2,294 \times 10^{-3}$ & 6,9019 & $4,61 \times 10^{-4}$ & 0,2023 \\
\hline
\end{tabular}




\subsubsection{Amostragem Melhorada}

O mesmo procedimento adotado na análise da técnica de Amostragem Assintótica é utilizado para realizar a análise da técnica de Amostragem Melhorada. De acordo com a Figura 58, para uma amostra de tamanho $3 \times 10^{3}$, e com a Figura 59, para uma amostra de tamanho $1 \times 10^{5}$ se observa o ajuste não linear obtido. Nesse caso foram utilizados 100 pontos de suporte $\operatorname{com} \lambda$ variando de 0,4 a 0,9 .

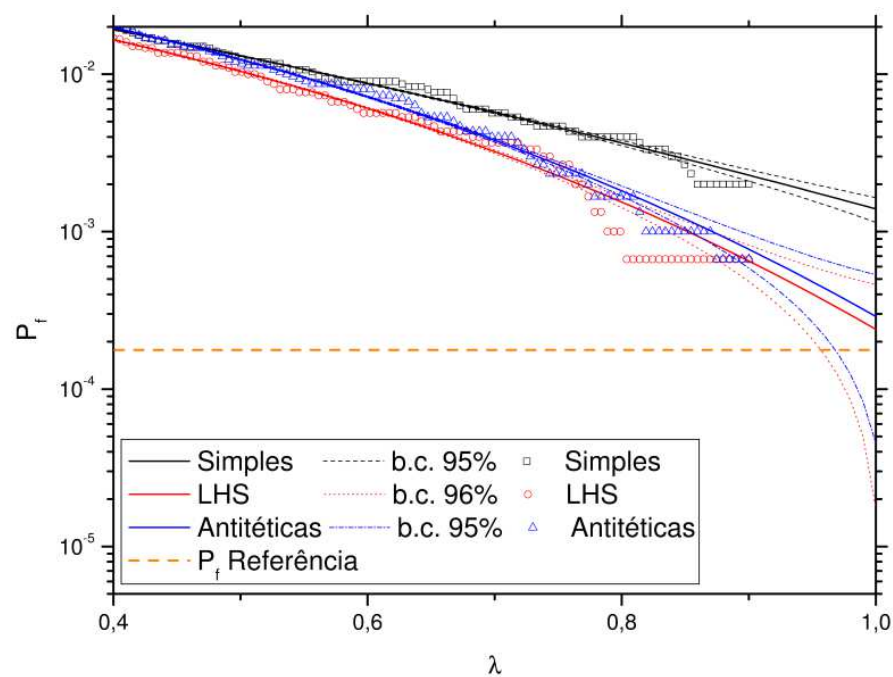

Figura 58. Regressão não linear utilizada na Amostragem Melhorada para amostras de tamanho $3 \times 10^{3}$, no Exemplo 3.

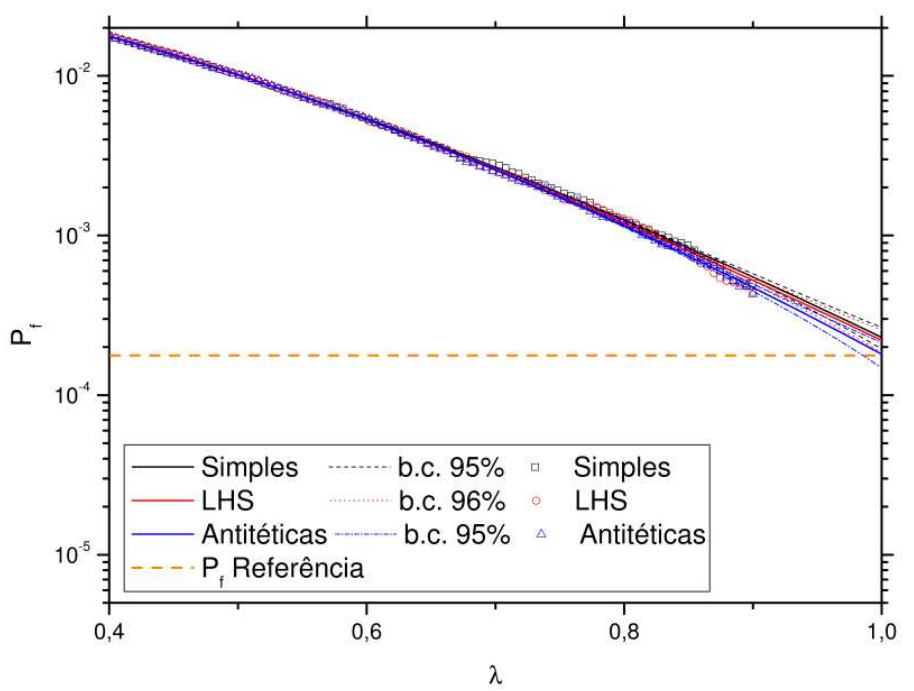

Figura 59. Regressão não linear utilizada na Amostragem Melhorada para amostras de tamanho $1 \times 10^{5}$, no Exemplo 3. 
Os parâmetros das funções ajustadas para a técnica em questão, com Amostragem Simples (AM), com Amostragem por Hipercubo Latino (AM_LHS) e com a Amostragem por Variáveis Antitéticas (AAM), são apresentados na Tabela 15, para uma amostra de tamanho $3 \times 10^{3}$ e na Tabela 16, para uma amostra de tamanho $1 \times 10^{5}$.

Tabela 15. Parâmetros ajustados na Amostragem Melhorada, para uma amostra de tamanho $3 \times 10^{3}$, no Exemplo 3.

\begin{tabular}{ccccc}
\hline & $\boldsymbol{q}$ & $\boldsymbol{a}$ & $\boldsymbol{b}$ & $\boldsymbol{c}$ \\
\hline AM & 4,6704 & $1,3127 \times 10^{-3}$ & $-6,1297$ & 4,4442 \\
AM_LHS & 0,18293 & $3,2094 \times 10^{-10}$ & $-6,3378$ & 11,918 \\
AAM & 0,19760 & $1,8992 \times 10^{-4}$ & $-3,3776$ & 7,0742 \\
\hline
\end{tabular}

Tabela 16. Parâmetros ajustados na Amostragem Melhorada, para uma amostra de tamanho $1 \times 10^{5}$, no Exemplo

\begin{tabular}{|c|c|c|c|c|}
\hline & $q$ & $a$ & $b$ & $\boldsymbol{c}$ \\
\hline $\mathbf{A M}$ & $4,3569 \times 10^{-2}$ & 6,5821 & 0,14392 & 1,4627 \\
\hline AM_LHS & $2,4595 \times 10^{-2}$ & 7,6629 & 0,31445 & 1,2864 \\
\hline $\mathbf{A} \overline{\mathbf{A}} \mathbf{M}$ & $5,6836 \times 10^{-2}$ & 5,7264 & $-2,4715 \times 10^{-3}$ & 1,7407 \\
\hline
\end{tabular}

A Tabela 17 apresenta as probabilidades de falha e seus coeficientes de variação, para diferentes tamanhos da amostra e para o uso de Amostragem Simples, da Amostragem por Hipercubo Latino e da Amostragem por Variáveis Antitéticas.

Tabela 17. Probabilidade de falha e coeficiente de Variação da probabilidade de falha, para a Amostragem Melhorada, no Exemplo 3.

\begin{tabular}{ccccc}
\hline \multicolumn{5}{c}{$\boldsymbol{P}_{\boldsymbol{f}}$ de referência: $\mathbf{1 , 7 7 4 \times \mathbf { 1 0 } ^ { - 4 }}$} \\
\hline $\boldsymbol{N}$ & $\boldsymbol{P}_{\boldsymbol{f}}$ & $\boldsymbol{C} \times \mathbf{1 0}^{\mathbf{3}} \boldsymbol{V}$. & $\boldsymbol{P}_{\boldsymbol{f}}$ & $\boldsymbol{1 \times 1 0 ^ { \mathbf { 5 } }}$ \\
& $1,394 \times 10^{-3}$ & 0,0915 & $2,300 \times 10^{-4}$ & 0,0791 \\
AM & $2,392 \times 10^{-4}$ & 0,4729 & $2,204 \times 10^{-4}$ & 0,0831 \\
AM_LHS & $2,889 \times 10^{-4}$ & 1,9724 & $1,807 \times 10^{-4}$ & 0,0929 \\
AAM &
\end{tabular}

\subsubsection{Simulação de Subconjuntos}

Os dados de entrada do StRAnD, que definem o caminhante aleatório utilizado na Simulação de Subconjuntos, são apresentados na Tabela 18.

Tabela 18. Médias e desvios padrão do caminhante aleatório do Exemplo 3.

\begin{tabular}{clll}
\hline Variável Aleatória & Distribuição & Média & Desvio padrão \\
\hline $\mathbf{X}_{\mathbf{1}}$ & Uniforme & 0,0 & $\alpha \times 1,0$ \\
$\mathbf{X}_{\mathbf{2}}$ & Uniforme & 0,0 & $\alpha \times 1,0$ \\
\hline
\end{tabular}

Na Figura 60, na Figura 61 e na Figura 62 podem-se observar quatro subconjuntos que foram gerados na Simulação de Subconjuntos. Considera-se uma amostra de tamanho 1.500 
para cada subconjunto, com $\alpha=0,2$ e $P_{0}=0,1$. Neste caso, pode-se observar uma boa concordância da técnica com o que ela propõe realizar.

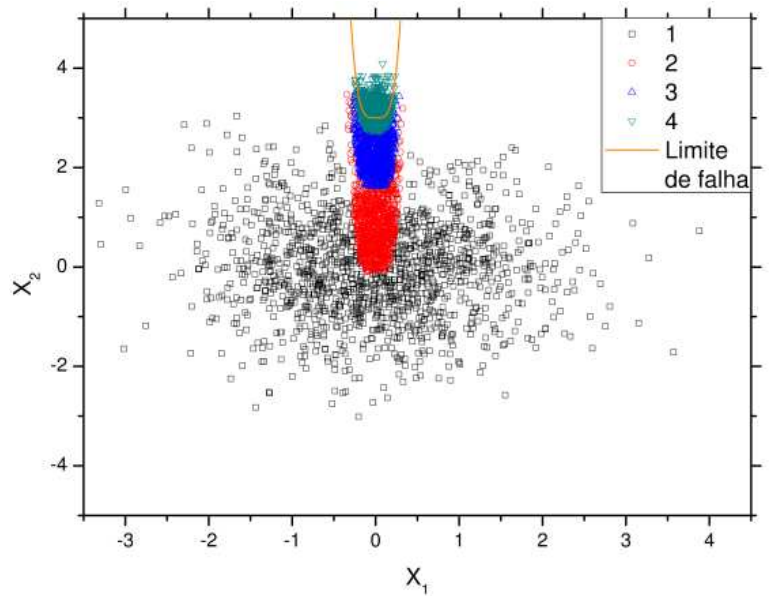

Figura 60. Simulação de Subconjuntos: Amostragem Simples, no Exemplo 3.

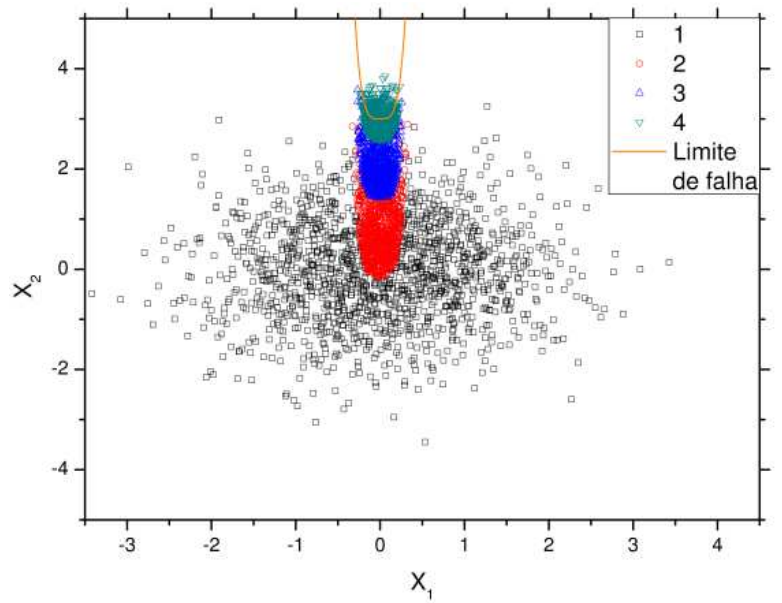

Figura 61. Simulação de Subconjuntos: Amostragem por Hipercubo Latino, no Exemplo 3.

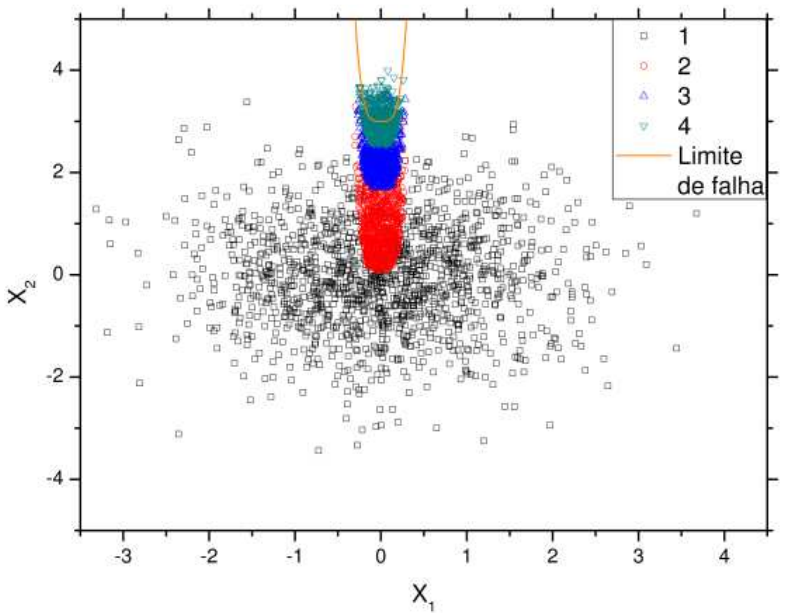

Figura 62. Simulação de Subconjuntos: Amostragem por Variáveis Antitéticas, no Exemplo 3. 


\subsubsection{Análise de convergência}

É realizado um comparativo entre o Monte Carlo Bruto, a Amostragem por Importância, a Amostragem Assintótica e a Amostragem Melhorada, tal como apresentado na Figura 63, na Figura 64 e na Figura 65. Variou-se o tamanho da amostra de $3 \times 10^{3}$ até $1 \times 10^{5}$, tomando nesse intervalo 100 pontos. Além disso, utiliza-se a Amostragem Simples, a Amostragem por Hipercubo Latino e a Amostragem com Variáveis Antitéticas.

a) Média

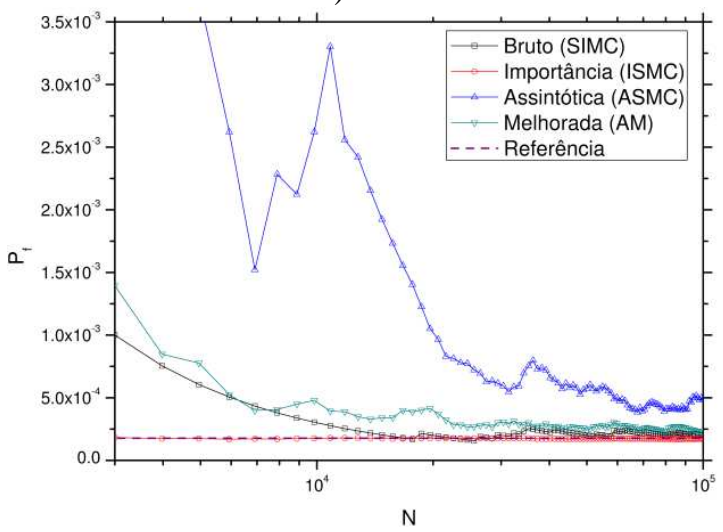

b) C.V.

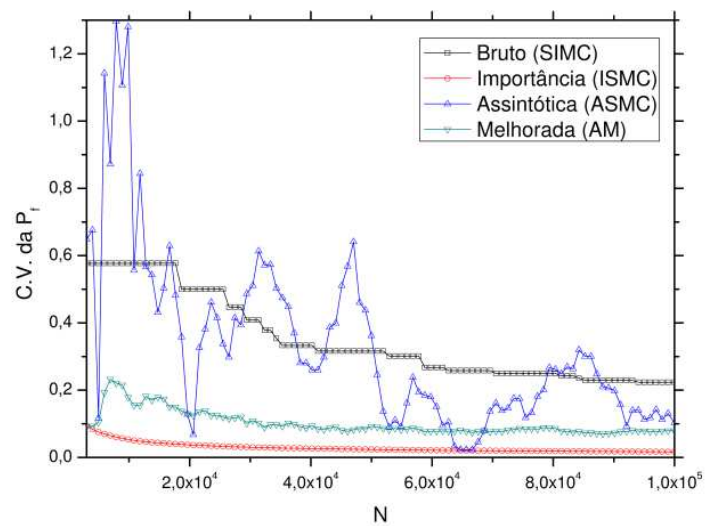

Figura 63. Comparativo da convergência da $P_{f}($ a) e de C.V. (b) para a Amostragem Simples, no Exemplo 3.

a) Média

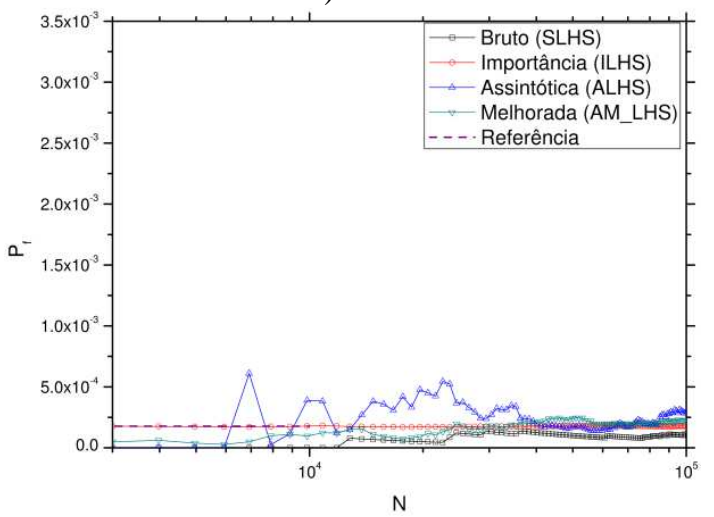

b) C.V.

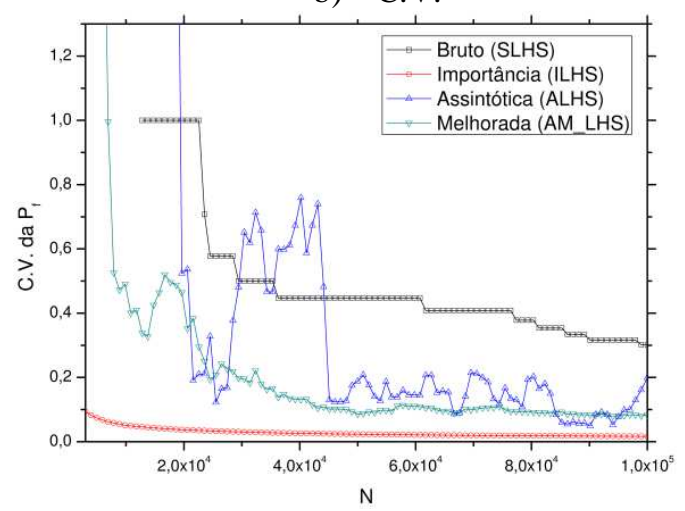

Figura 64. Comparativo da convergência da $P_{f}($ a) e de C.V. (b) para a Amostragem por Hipercubo Latino, no Exemplo 3. 
a) Média

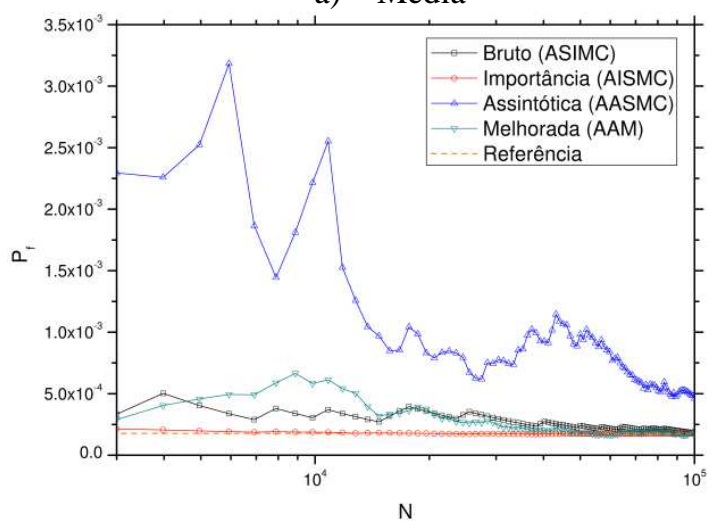

b) C.V.

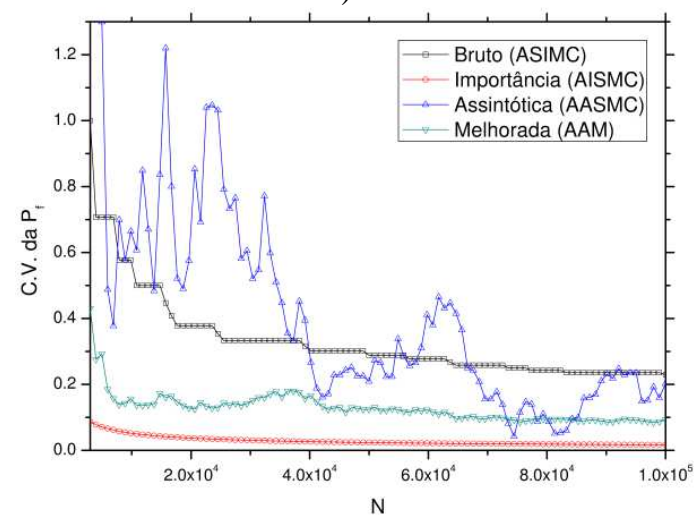

Figura 65. Comparativo da convergência da $P_{f}($ a) e de C.V. (b) para a Amostragem por Variáveis Antitéticas, no Exemplo 3.

Pode-se notar que a Amostragem Assintótica não apresenta um bom resultado em termos da convergência da probabilidade de falha e de seu coeficiente de variação. Por outro lado, a Amostragem por Importância é a que apresenta o melhor resultado, como era esperado. Também se observa que o Monte Carlo Bruto apresentou resultados consistentes. Apesar da Amostragem Melhorada apresentar uma redução do coeficiente de variação da probabilidade de falha, em comparação com o Monte Carlo Bruto e com a Amostragem Assintótica, a convergência da probabilidade de falha é muito próxima da convergência obtida pelo Monte Carlo Bruto. Por outro lado, utilizando a Amostragem por Hipercubo Latino, a Amostragem Melhorada apresentou um melhor desempenho.

Nos graficos apresentados na Figura 66, na Figura 67, na Figura 68 e na Figura 69, é possível observar a influência da utilização da Amostragem Simples, da Amostragem por Hipercubo Latino, da Amostragem por Variáveis Antitéticas para o Monte Carlo Bruto, para a Amostragem por Importância, para a Amostragem Assintótica e para a Amostragem Melhorada. Nestes gráficos são adotados 100 pontos para a análise da convergência.

a) Média

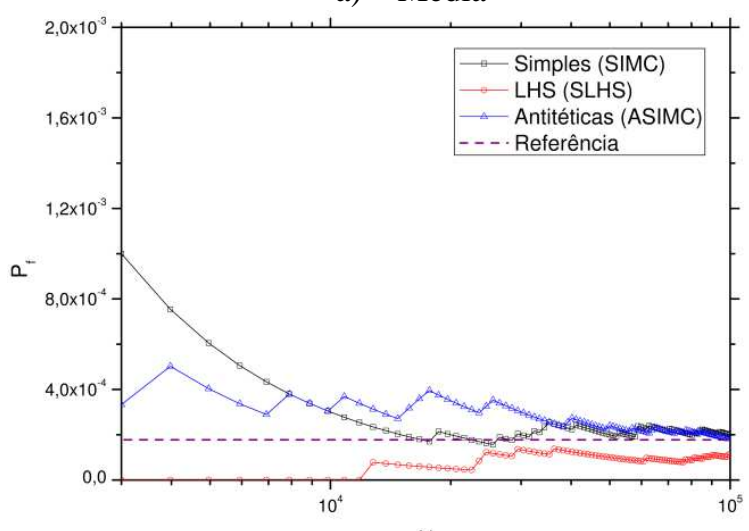

b) C.V.

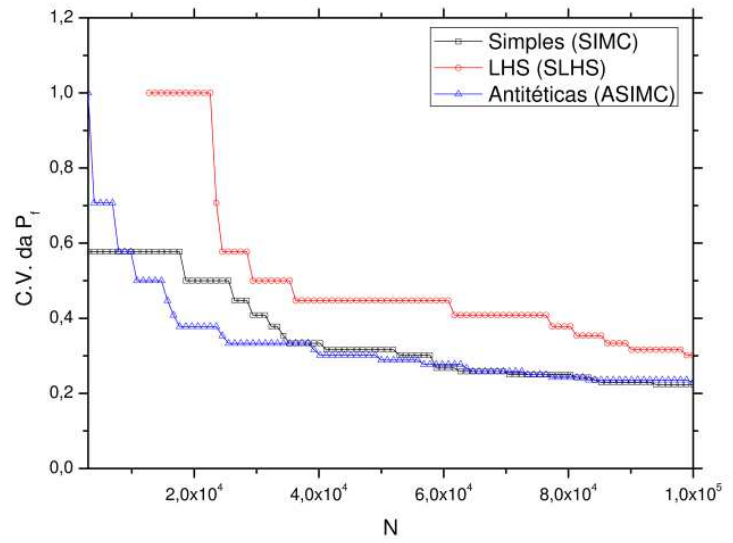

Figura 66. Comparativo da convergência da $P_{f}$ (a) e de C.V. (b) para o Monte Carlo Bruto, no Exemplo 3. 
a) Média

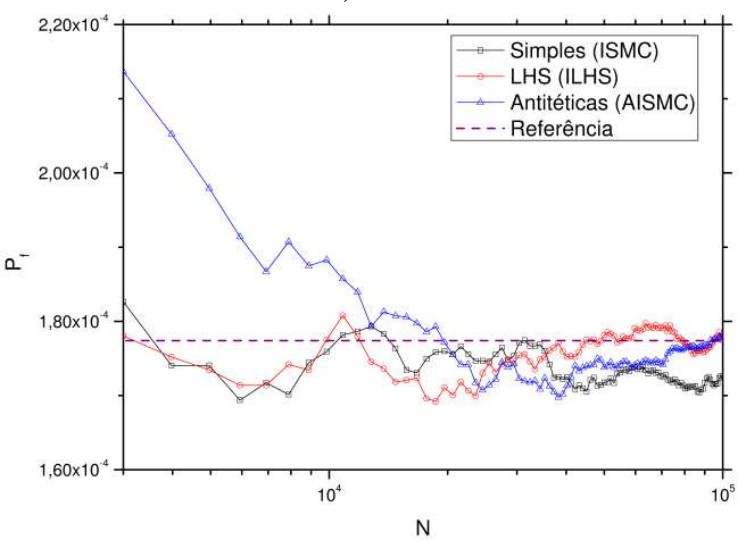

b) C.V.

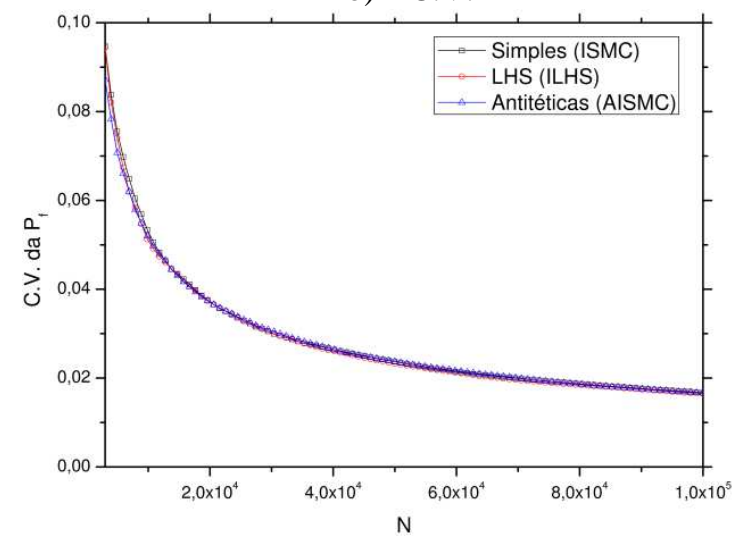

Figura 67. Comparativo da convergência da $P_{f}$ (a) e de C.V. (b) para a Amostragem por Importância, no Exemplo 3.

a) Média

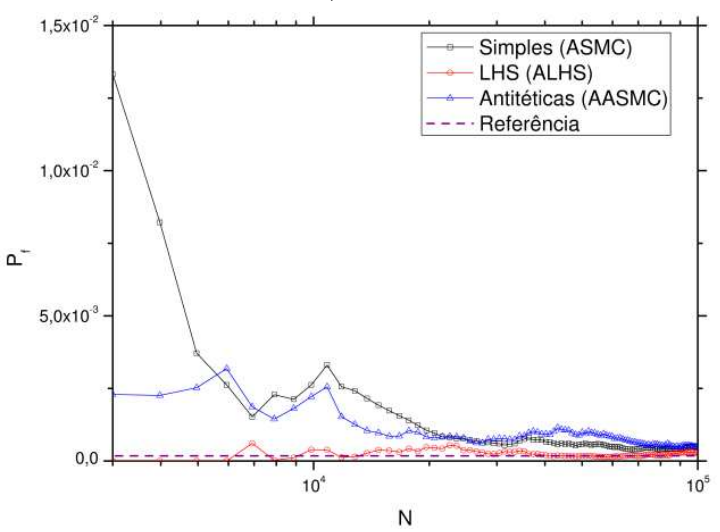

b) C.V.

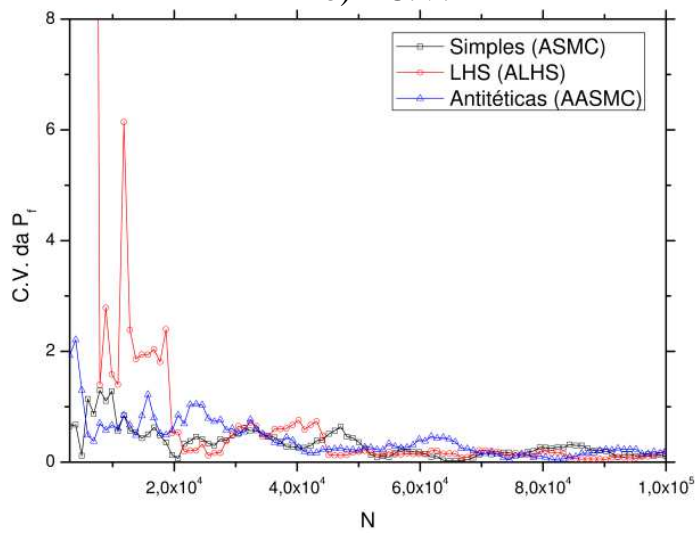

Figura 68. Comparativo da convergência da $P_{f}$ (a) e de C.V. (b) para a Amostragem Assintótica, no Exemplo 3.

a) Média

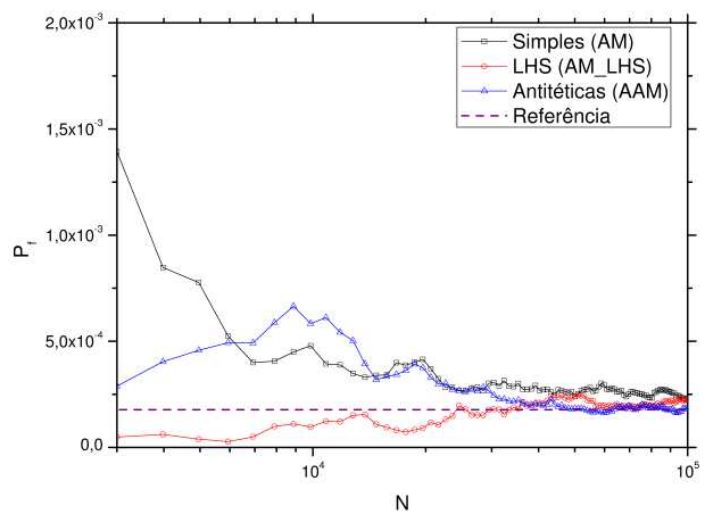

b) C.V.

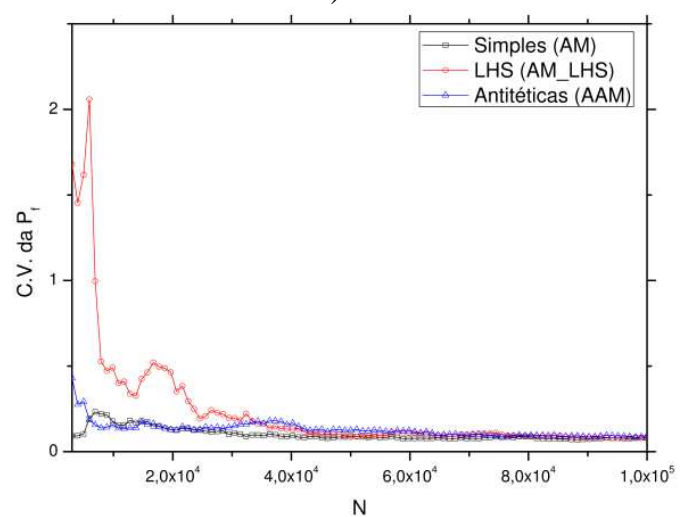

Figura 69. Comparativo da convergência da $P_{f}$ (a) e de C.V. (b) para a Amostragem Melhorada, no Exemplo 3. 
Observa-se no Monte Carlo Bruto, que nem a Amostragem por Hipercubo Latino, nem a Amostragem por Variáveis Antitéticas, levaram a uma melhora significativa na convergencia da probabilidade de falha (Figura 66a). Já a Amostragem por Hipercubo Latino apresentou um coeficiente de variação da probabilidade de falha (Figura 66b) maior que as outras técnicas as quais ele foi comparado. Na Amostragem por Importância, observa-se que o uso da Amostragem por Variáveis Antitéticas apresenta uma influência negativa na probabilidade de falha, nas primeiras simulações. $O$ uso da Amostragem por Hipercubo Latino e por Variáveis Antitéticas, levou a uma melhora da convergência da probabilidade de falha (Figura 68a) na técnica de Amostragem Assintótica. Porém, tal melhora não se reflete no coeficiente de variação (Figura 68b) da probabilidade de falha. Em relação a Amostragem Melhorada, observa-se que esta apresenta um um bom resultado quando utilizada em conjunto com a Amostragem por Hipercubo Latino e Amostragem por Variáveis Antitéticas (Figura 69).

\subsubsection{Comparativo da probabilidade de falha e de seu coeficiente de variação}

Neste exemplo é utilizada uma amostra de tamanho 5.550. Tais resultados podem ser observados na Tabela 19. Na técnica de Simulação de Subconjuntos adotam-se os seguintes parâmetros: $N_{S S}=1.500, \alpha=0,2, P_{0}=0,1$. O caminhante aleatório segue uma distribuição de probabilidade uniforme. 
Tabela 19. Comparativo da $P_{f}$ para uma amostra de tamanho 5.500, no Exemplo 3.

\begin{tabular}{|c|c|c|c|c|}
\hline \multicolumn{5}{|c|}{$P_{f}$ de referência: $1,774 \times 10^{-4}$} \\
\hline \multicolumn{5}{|c|}{ SIMPLES } \\
\hline Sigla & Técnica & $\boldsymbol{P}_{f}$ & C.V.da $P_{f}$ & Erro $(\%)$ \\
\hline BRUTO & Bruto & $5,405 \times 10^{-4}$ & 0,5772 & 204,68 \\
\hline AI & Importância & $1,701 \times 10^{-4}$ & 0,0717 & 4,11 \\
\hline $\mathbf{A A}$ & Assintótica & $2,873 \times 10^{-3}$ & 0,4744 & 1519,50 \\
\hline $\mathbf{A M}$ & Melhorada & $5,562 \times 10^{-4}$ & 0,1858 & 213,53 \\
\hline SS & Subconjuntos & $4,480 \times 10^{-4}$ & 0,2845 & 152,54 \\
\hline \multicolumn{5}{|c|}{ HIPERCUBO LATINO } \\
\hline Sigla & Técnica & $P_{f}$ & C.V.da $P_{f}$ & Erro $(\%)$ \\
\hline BRUTO & Bruto & -- & -- & -- \\
\hline AI & Importância & $1,883 \times 10^{-4}$ & 0,0688 & 6,14 \\
\hline $\mathbf{A A}$ & Assintótica & $1,140 \times 10^{-4}$ & 4,7053 & 35,74 \\
\hline $\mathbf{A M}$ & Melhorada & $5,672 \times 10^{-5}$ & 1,3958 & 73,66 \\
\hline SS & Subconjuntos & $1,860 \times 10^{-4}$ & 0,3099 & 4,85 \\
\hline \multicolumn{5}{|c|}{ VARIÁVEIS ANTITÉTICAS } \\
\hline Sigla & Técnica & $\boldsymbol{P}_{f}$ & C.V.da $P_{f}$ & Erro $(\%)$ \\
\hline BRUTO & Bruto & $3,604 \times 10^{-4}$ & -- & 103,16 \\
\hline AI & Importância & $1,903 \times 10^{-4}$ & 0,0684 & 7,27 \\
\hline $\mathbf{A A}$ & Assintótica & $5,031 \times 10^{-3}$ & 0,5638 & 2735,96 \\
\hline $\mathbf{A M}$ & Melhorada & $3,494 \times 10^{-4}$ & 0,2893 & 96,96 \\
\hline SS & Subconjuntos & $2,220 \times 10^{-4}$ & 0,3003 & 25,14 \\
\hline
\end{tabular}

Neste caso, nota-se que o uso de Amostragem por Importância é superior às outras técnicas, tal resultado já era esperado. Porém, é interessante notar que a técnica de Simulação de Subconjuntos apresenta um desempenho satisfatório tanto em termos de probabilidade de falha, quanto em termos do seu coeficiente de variação. Destaca-se que a maioria das técnicas é beneficiada pelo uso da Amostragem por Hipercubo Latino. Tal resultado pode ser conferido graficamente na Figura 70.

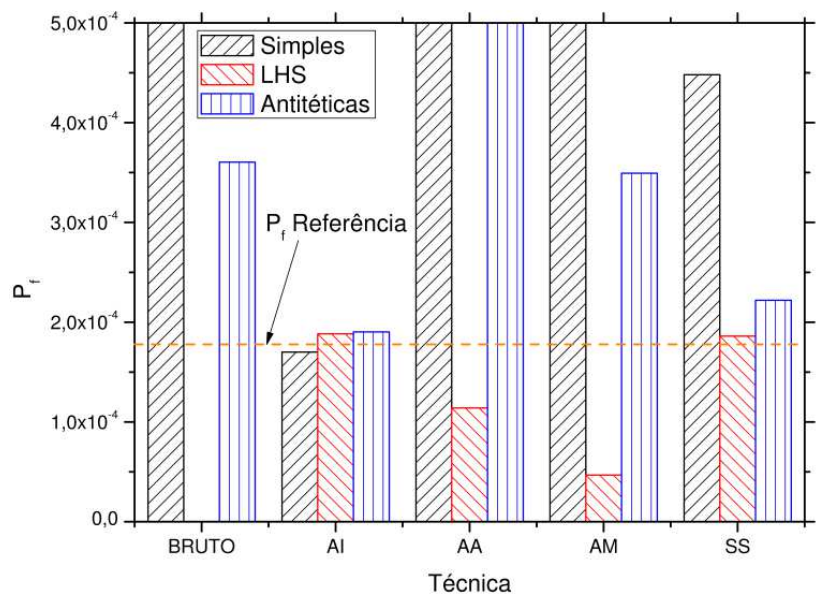

Figura 70. Comparativo da probabilidade de falha para uma amostra de tamanho 5.550, no Exemplo 3. 


\subsubsection{Tempo de processamento}

Nesta seção são apresentados os gráficos da relação entre o tempo de processamento e o tamanho da amostra. Na Figura 71 e na Figura 72, comparam-se os tempos de processamento do Monte Carlo Bruto (BRUTO), do Monte Carlo com Amostragem por Importância (AI), da Amostragem Assintótica (AA) e da Amostragem Melhorada (AM). É feito uso da Amostragem Simples, da Amostragem por Hipercubo Latino e da Amostragem por Variáveis Antitéticas. O tamanho da amostra varia de $1 \times 10^{5}$ até $9 \times 10^{5}$. Já na Figura $73 a$, comparam-se os tempos de processamento da Simulação de Subconjuntos (SS) e na Figura 73b, comparamse os tempos de processamento de todas as técnicas estudadas neste trabalho. Na Figura 73a e na Figura 73b, o tamanho da amostra varia de 7.400 até 37.000. Para a Simulação de Subconjuntos, consideram-se os mesmos parâmetros utilizados no estudo comparativo apresentado em 7.3.5, com o tamanho de cada subconjunto variando de 2.000 até 10.000 com um intervalo de 2.000 entre cada tamanho de subconjunto.

a) Monte Carlo Bruto

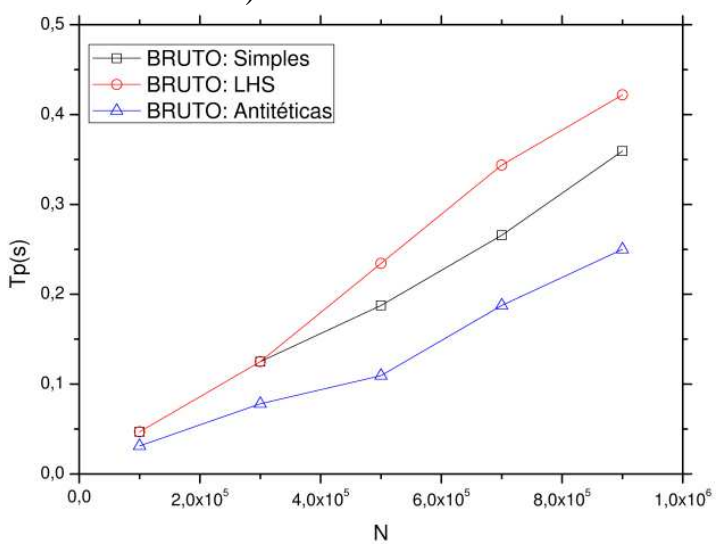

b) Amostragem por Importância

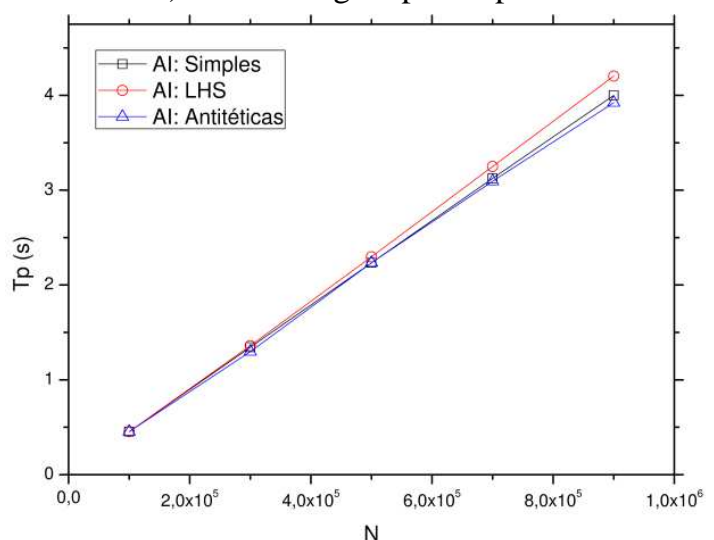

Figura 71. Relação entre o tempo de processamento e o tamanho da amostra no Exemplo 3: Monte Carlo Bruto (a) e Amostragem por Importância (b). 
a) Amostragem Assintótica

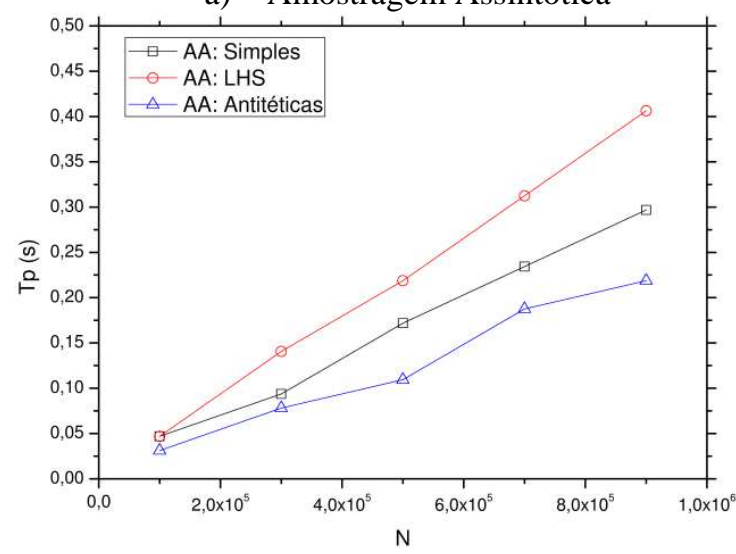

b) Amostragem Melhorada

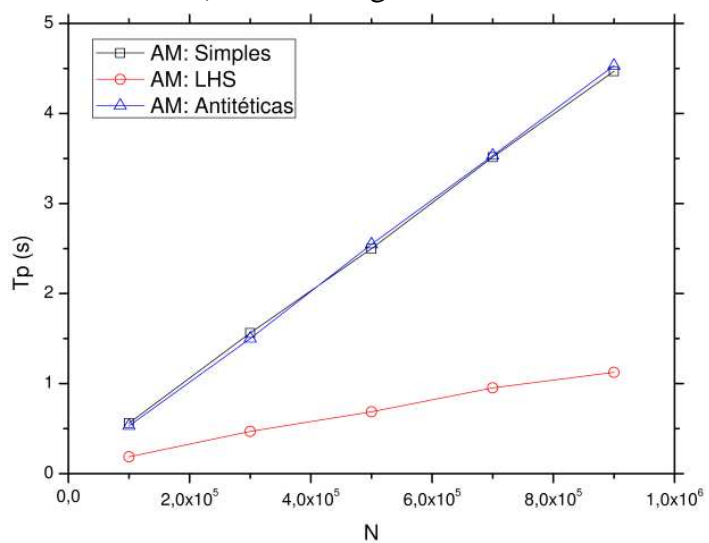

Figura 72. Relação entre o tempo de processamento e o tamanho da amostra no Exemplo 3: Amostragem Assintótica (a) e Amostragem Melhorada (b).

a) Simulação de Subconjunto

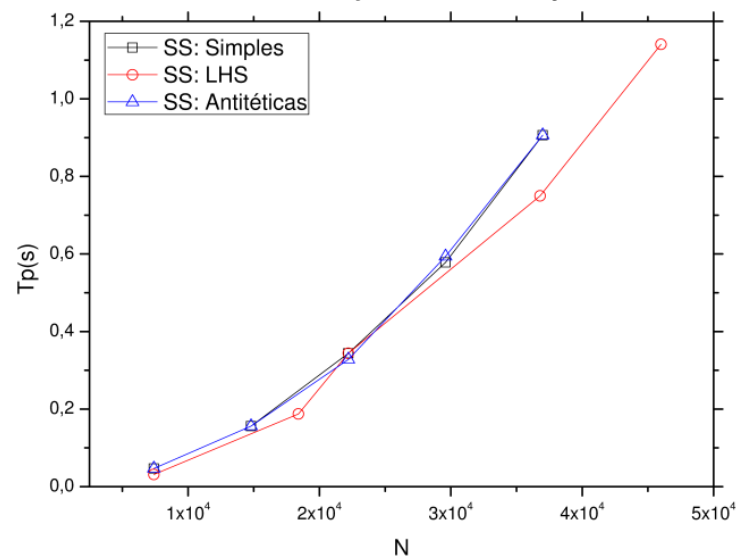

b) Todas as técnicas

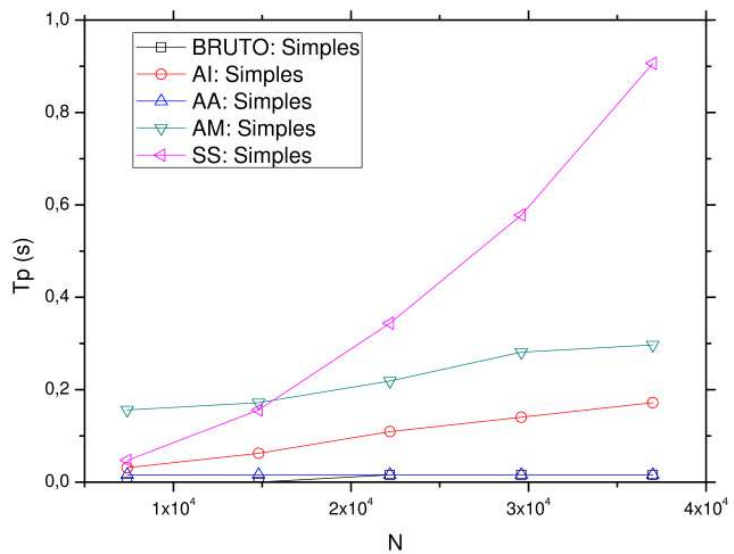

Figura 73. Relação entre o tempo de processamento e o tamanho da amostra no Exemplo 3: Simulação de Subconjuntos (a) e comparativo entre todas as técnicas(b).

A análise que pode ser feita neste problema é a mesma que foi realizada no Exemplo 1, pois trata-se de um problema com duas variáveis aleatórias, da mesma forma que no Exemplo 1. Observa-se que a não linearidade da equação de estado limite não apresenta influência significativa no tempo de processamento. Além disso, a defasagem do tamanho da amostra em cada ponto do gráfico da Figura 73a, é devido a adaptatividade da técnica, pois nesta o usuário tem o controle do tamanho da amostra em cada subconjunto e não da amostra total.

\subsection{Exemplo 4: Equação de estado limite não linear com variáveis aleatórias mistas}

Este problema é uma adaptação do exemplo apresentado por Melchers e Ahammed (2004). Aqui o objetivo é analisar um problema que possui uma equação de estado limite não linear, cujas variáveis aleatórias são modeladas a partir de diferentes funções de densidade de 
probabilidade. A adaptação adotada na equação de estado limite (Eq. (142)), consiste na aplicação da constante $A=2,0$, cujo objetivo é reduzir o valor da probabilidade de falha do problema apresentado por Melchers e Ahammed (2004).

$$
g\left(\mathrm{X}_{1}, \mathrm{X}_{2}, \mathrm{X}_{3}, \mathrm{X}_{4}, \mathrm{X}_{5}, \mathrm{X}_{6}\right)=A \cdot \mathrm{X}_{1} \cdot \mathrm{X}_{2} \cdot \mathrm{X}_{3} \cdot \mathrm{X}_{4}-\frac{\mathrm{X}_{5} \cdot X_{6}^{2}}{8}
$$

Este problema busca analisar uma situação extrema, em que existe uma equação de estado limite não linear com várias variáveis aleatórias, sendo tais variáveis aleatórias definidas por diferentes distribuições de probabilidade. Os parâmetros das distribuições de probabilidade das variáveis aleatórias são apresentados na Tabela 20.

Tabela 20. Médias e desvios padrão das variáveis aleatórias do Exemplo 4.

\begin{tabular}{clll}
\hline Variável Aleatória & Distribuição & Média & Desvio padrão \\
\hline $\boldsymbol{X}_{\mathbf{1}}$ & Weibull (mínima) & 4,0 & 0,1 \\
$\boldsymbol{X}_{\mathbf{2}}$ & Log-normal & $25.000,0$ & $2.000,0$ \\
$\boldsymbol{X}_{\mathbf{3}}$ & Gumbel & 0,875 & 0,1 \\
$\boldsymbol{X}_{\mathbf{4}}$ & Uniforme & 20,0 & 1,0 \\
$\boldsymbol{X}_{\mathbf{5}}$ & Exponencial & 100,0 & 100,0 \\
$\boldsymbol{X}_{\mathbf{6}}$ & Normal & 150,0 & 10,0 \\
\hline
\end{tabular}

Neste caso a probabilidade de falha obtida por meio do FORM foi de $3,245 \times 10^{-5}$ $(\beta=3,9942)$. Porém, como a equação de estado limite deste problema é não linear, não se recomenda o uso do FORM para estimar a probabilidade de falha. Com uma amostra de tamanho $2 \times 10^{9}$ e utilizando o método de Monte Carlo Bruto com Amostragem Simples, se obtém uma probabilidade de falha de $2,777 \times 10^{-5}(\beta=4,031)$ com um coeficiente de variação de 0,0042 .

A seguir serão apresentados os estudos referentes as técnicas de Amostragem Assintótica e de Amostragem Melhorada.

\subsubsection{Amostragem Assintótica}

Nesta seção é realizado um estudo da aplicação da técnica de Amostragem Assintótica no problema em questão. Utilizam-se na Amostragem Assintótica, a Amostragem Simples, a Amostragem por Hipercubo Latino e a Amostragem por Variáveis Antitéticas. Considera-se uma amostra de tamanho $1 \times 10^{4}$ (Figura 74) e outra de tamanho $1 \times 10^{5}$ (Figura 75). O parâmetro $f$ é variado de 0,4 a 0,7 , onde são utilizados 5 pontos de suporte. Neste caso é adotada a Eq. (109), como função a ser ajustada. 


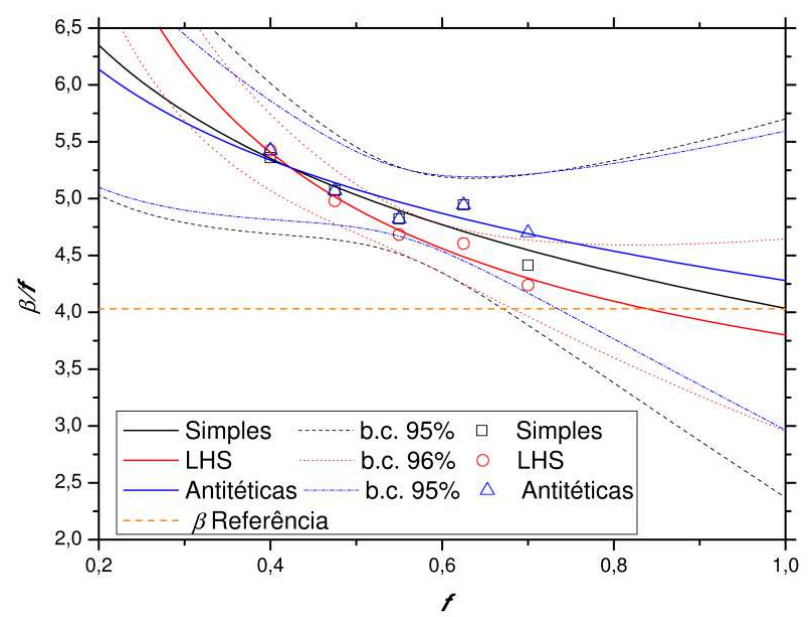

Figura 74. Regressão não linear utilizada na Amostragem Assintótica para amostras de tamanho $1 \times 10^{4}$, no Exemplo 4.

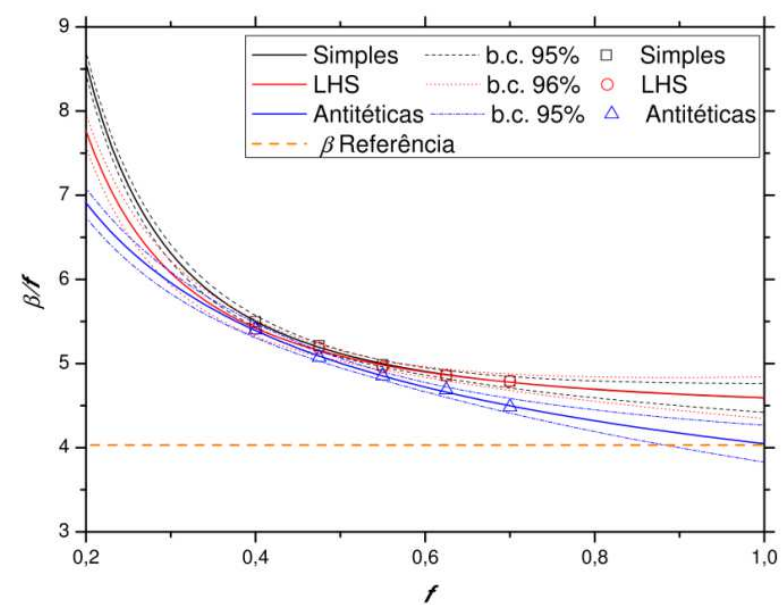

Figura 75. Regressão não linear utilizada na Amostragem Assintótica para amostras de tamanho $1 \times 10^{5}$, no Exemplo 4.

De acordo com a Figura 74 e com a Figura 75, pode-se observar certa concordância entre o modelo proposto no ajuste e os pontos de suporte obtidos por simulações de Monte Carlo, apesar de se notar diferenças entre o valor exato do índice de confiabilidade e aquele obtido por meio da Amostragem Assintótica.

Os parâmetros ajustados na técnica em questão, com a Amostragem Simples (ASMC), com a Amostragem por Hipercubo Latino (ALHS) e com a Amostragem por Variáveis Antitéticas (AASMC), são apresentados na Tabela 21, para as amostras de tamanho $1 \times 10^{4} \mathrm{e}$ de tamanho $1 \times 10^{5}$. 
Tabela 21. Parâmetros ajustados na Amostragem Assintótica, para o Exemplo 4.

\begin{tabular}{ccccccc}
\hline $\boldsymbol{N}$ & \multicolumn{3}{c}{$\mathbf{1 \times 1 0}^{\mathbf{4}}$} & \multicolumn{4}{c}{$\mathbf{1 \times \mathbf { 1 0 } ^ { \mathbf { 5 } }}$} \\
\hline & $\boldsymbol{A}$ & $\boldsymbol{B}$ & $\boldsymbol{C}$ & $\boldsymbol{A}$ & $\boldsymbol{B}$ & $\boldsymbol{C}$ \\
ASMC & $-5.330,211$ & $5.334,246$ & $-2,695 \times 10^{-4}$ & 4,403 & 0,1887 & $-1,922$ \\
ALHS & 2,155 & 1,645 & $-0,744$ & 4,373 & 0,221 & $-1,696$ \\
AASMC & $12.391,608$ & $-12.387,328$ & $9,324 \times 10^{-5}$ & 1,753 & 2,295 & $-0,503$ \\
\hline
\end{tabular}

Realizando um maior número de simulações, a aderência entre os pontos de suporte e a curva ajustada melhora significativamente. O comparativo da probabilidade de falha e de seu coeficiente de variação, para diferentes tamanhos de amostra e para as diferentes técnicas de amostragem básica, são apresentados na Tabela 22.

Tabela 22. Probabilidade de falha e coeficiente de Variação da probabilidade de falha, para a Amostragem Assintótica, no Exemplo 4.

\begin{tabular}{ccccc}
\hline \multicolumn{5}{c}{$\boldsymbol{P}_{\boldsymbol{f}}$ de referência: $\mathbf{2 , 7 7 7 \times \mathbf { 1 0 }}$} \\
\hline $\boldsymbol{N}$ & $\mathbf{1 \times \mathbf { 1 0 } ^ { \mathbf { 4 } }}$ & $\mathbf{1 \times 1 0}^{\mathbf{5}}$ \\
& $P_{f}$ & $C . V$. & $P_{f}$ & $C . V$. \\
ASMC & $2,724 \times 10^{-5}$ & 165,7952 & $2,196 \times 10^{-6}$ & 0,6346 \\
ALHS & $7,23 \times 10^{-5}$ & 10,5330 & $2,175 \times 10^{-6}$ & 1,1075 \\
AASMC & $9,348 \times 10^{-6}$ & 82,0325 & $2,587 \times 10^{-5}$ & 0,7608 \\
\hline
\end{tabular}

De acordo com a Tabela 22, a técnica de Amostragem Assintótica apresentou uma boa resposta apenas no seu uso em conjunto com a Amostragem por Variáveis Antitéticas.

\subsubsection{Amostragem Melhorada}

Para o estudo da Amostragem Melhorada são utilizados 100 pontos de suporte, $\operatorname{com} \lambda$ variando de 0,4 a 0,9. É realizada a simulação da técnica de Amostragem Melhorada utilizando a Amostragem Simples, a Amostragem por Hipercubo Latino e a Amostragem por Variáveis Antitéticas. O estudo é realizado com uma amostra de tamanho $10^{4}$ e outra de tamanho $10^{5}$.

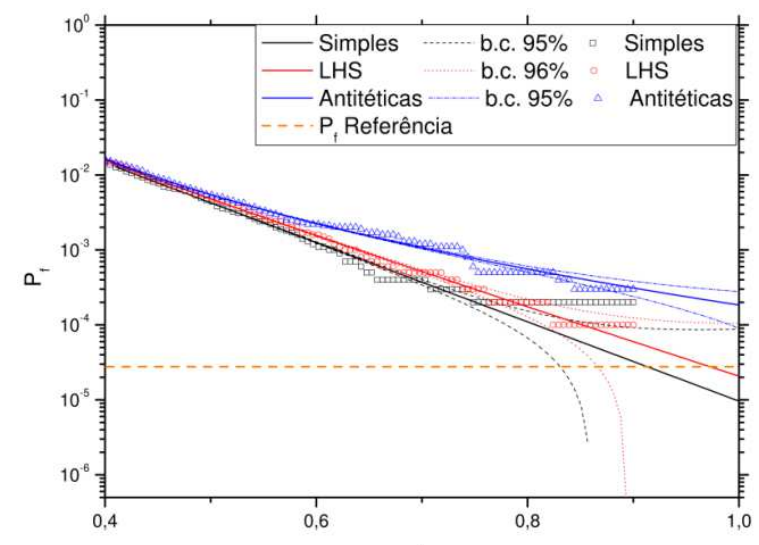

Figura 76. Regressão não linear utilizada na Amostragem Melhorada para amostras de tamanho $10^{4}$, no Exemplo 4. 


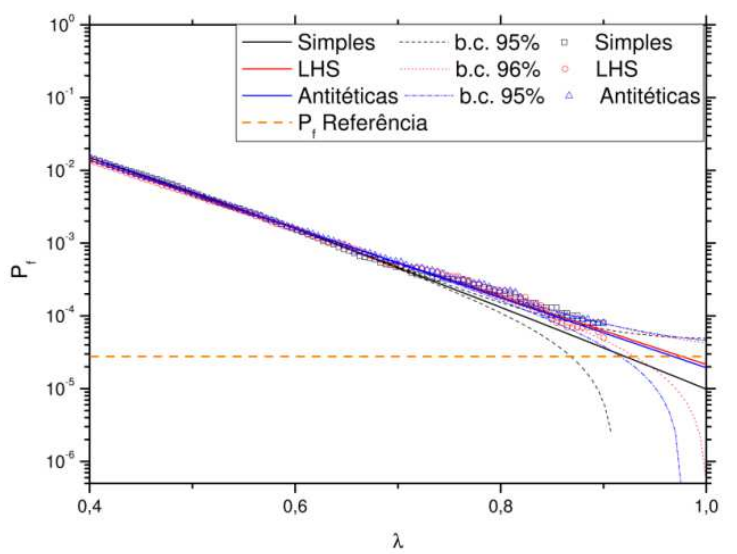

Figura 77. Regressão não linear utilizada na Amostragem Melhorada para amostras de tamanho $10^{5}$, no Exemplo 4.

Os parâmetros das funções ajustadas para a técnica em questão, com a Amostragem Simples (AM), com a Amostragem por Hipercubo Latino (AM_LHS) e com a Amostragem por Variáveis Antitéticas (AAM), são apresentados na Tabela 23, para uma amostra de tamanho $1 \times 10^{4}$ e na Tabela 24 , para uma amostra de tamanho $1 \times 10^{5}$.

Tabela 23. Parâmetros ajustados na Amostragem Melhorada, para uma amostra de tamanho $1 \times 10^{4}$, no Exemplo 4.

\begin{tabular}{ccccc}
\hline & $\boldsymbol{q}$ & $\boldsymbol{a}$ & $\boldsymbol{b}$ & $\boldsymbol{c}$ \\
\hline AM & $1,4543 \times 10^{-2}$ & 12,1800 & 0,4000 & 0,9969 \\
AM_LHS & $2,2041 \times 10^{-2}$ & 10,8720 & 0,3753 & 0,9542 \\
AAM & 879,72 & 16,1540 & 0,1647 & 0,2730 \\
\hline
\end{tabular}

Tabela 24. Parâmetros ajustados na Amostragem Melhorada, para uma amostra de tamanho $1 \times 10^{5}$, no Exemplo 4.

\begin{tabular}{ccccc}
\hline & $\boldsymbol{q}$ & $\boldsymbol{a}$ & $\boldsymbol{b}$ & $\boldsymbol{c}$ \\
\hline AM & $1,7665 \times 10^{-2}$ & 12,6060 & 0,3802 & 1,0881 \\
AM_LHS & $1,5571 \times 10^{-2}$ & 10,7370 & 0,3893 & 0,9915 \\
AAM & $1,4652 \times 10^{-2}$ & 11,0480 & 0,4000 & 0,9991 \\
\hline
\end{tabular}

A Tabela 25 apresenta as probabilidades de falha e seus coeficientes de variação, para diferentes tamanhos de amostra e para o uso da Amostragem Simples, da Amostragem por Hipercubo Latino e da Amostragem por Variáveis Antitéticas. 
Tabela 25. Probabilidade de falha e coeficiente de Variação da probabilidade de falha, para a Amostragem Melhorada, no Exemplo 4.

\begin{tabular}{ccccc}
\hline \multicolumn{5}{c}{$\boldsymbol{P}_{\boldsymbol{f}}$ de referência: $\mathbf{2 , 7 7 7 \times \mathbf { 1 0 } ^ { - 5 }}$} \\
\hline $\boldsymbol{N}$ & $\mathbf{1 \times 1 0}^{\mathbf{5}}$ & $\mathbf{1 \times 1 0 ^ { \mathbf { 5 } }}$ \\
& $P_{f}$ & $C . V$. & $P_{f}$ & $C . V$. \\
AM & $9,634 \times 10^{-6}$ & 4,1349 & $9,862 \times 10^{-6}$ & 2,0225 \\
AM_LHS & $2,074 \times 10^{-5}$ & 2,0713 & $2,151 \times 10^{-5}$ & 0,4975 \\
AAM & $1,842 \times 10^{-4}$ & 0,2604 & $1,944 \times 10^{-5}$ & 0,6943 \\
\hline
\end{tabular}

\subsubsection{Análise de convergência}

É realizado um comparativo entre o Monte Carlo Bruto, a Amostragem por Importância, a Amostragem Assintótica e a Amostragem Melhorada, todas utilizando a Amostragem Simples, a Amostragem por Hipercubo Latino e a Amostragem com Variáveis Antitéticas. O tamanho da amostra varia de $1 \times 10^{3}$ até $1 \times 10^{6}$, tomando nesse intervalo 100 pontos. Neste caso, o eixo das abscissas não está em escala logarítimica pelo fato de a escala decimal ter se apresentado melhor na análise deste problema.

a) Média

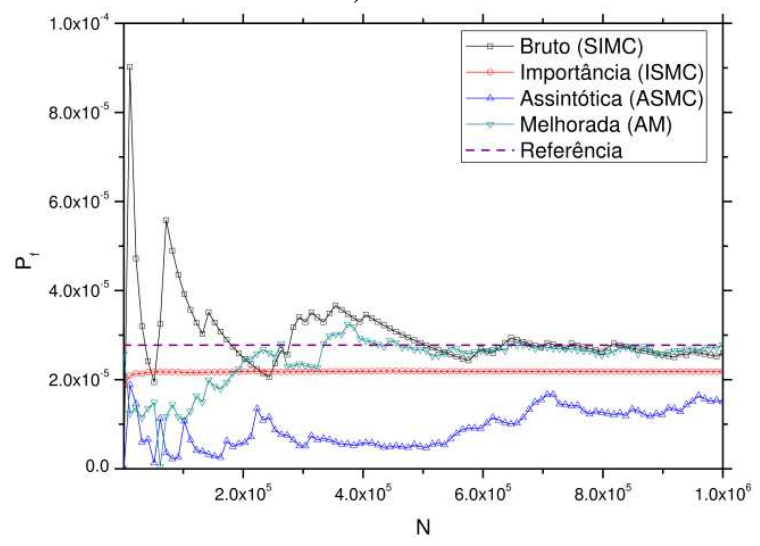

b) C.V.

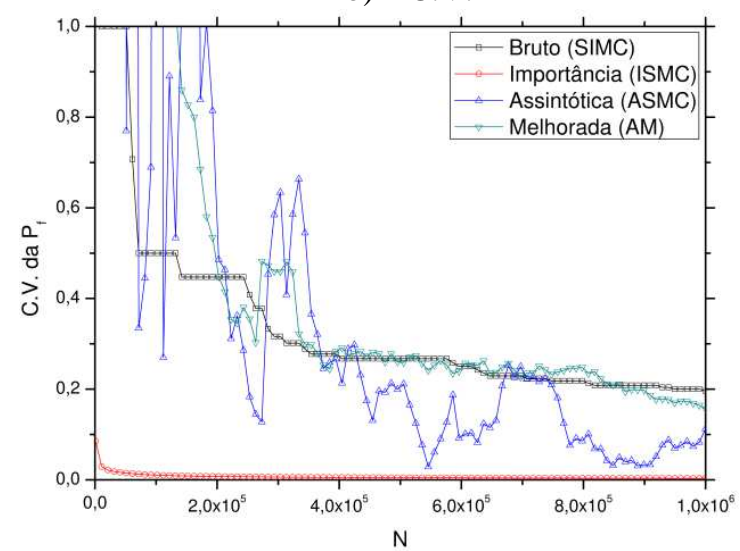

Figura 78. Comparativo da convergência da $P_{f}($ a) e de C.V. (b) para a Amostragem Simples, no Exemplo 4. 
a) Média

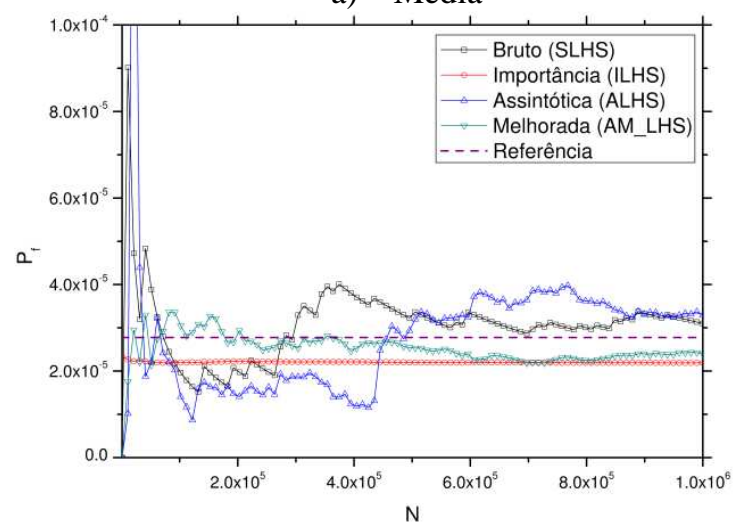

b) C.V.

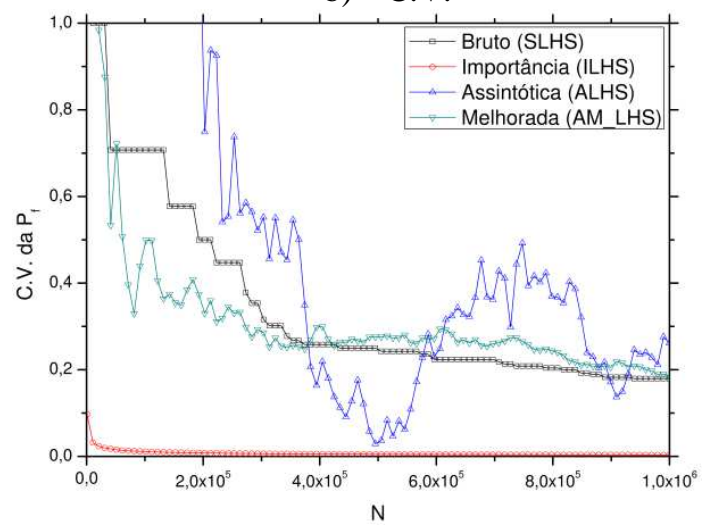

Figura 79. Comparativo da convergência da $P_{f}($ a) e de C.V. (b) para a Amostragem por Hipercubo Latino, no Exemplo 4.

a) Média

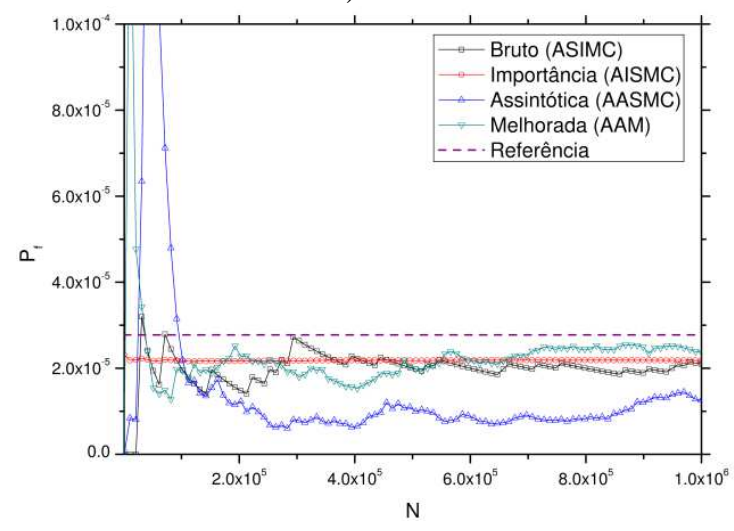

b) C.V.

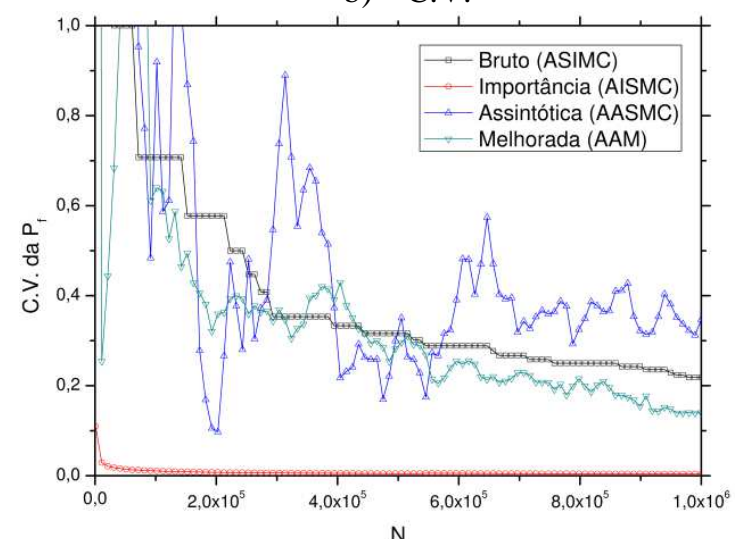

Figura 80. Comparativo da convergência da $P_{f}(a)$ e de C.V. (b) para a Amostragem por Variáveis Antitéticas, no Exemplo 4.

Na Figura 78a, a Amostragem por Importância e a Amostragem Assintótica não apresentam uma boa convergência da probabilidade de falha. Em relação a Amostragem por Importância, percebe-se um resultado tendencioso, mesmo com uma convergência rápida e com um baixo coeficiente de variação da probabilidade de falha (Figura 78b). Neste caso não se percebe vantagem significativa do uso das técnicas apresentadas, em relação ao Monte Carlo Bruto. Na Figura 79a, observa-se que a Amostragem por Importância ainda apresenta um resultado tedencioso, mesmo com a Amostragem por Hipercubo Latino. Por outro lado, é possível observar uma leve melhora no desempenho da Amostragem Melhorada apenas nas primeiras simulações, o que não reflete numa melhora do coeficiente de variação da probabilidade de falha (Figura 79b). Na Figura 80a e na Figura 80b, observa-se que não há uma melhora significativa do uso das técnicas em questão, em relação ao Monte Carlo Bruto. Tal dificuldade encontrada pelas técnicas comparadas acima pode ser devido ao uso de diferentes distribuições de probabilidade das variáveis aleatórias. 
Nos graficos apresentados a seguir é possível observar a influência da utilização da Amostragem por Hipercubo Latino e Amostragem por Variáveis Antitéticas, em comparação com a Amostragem Simples, no Monte Carlo Bruto, no Monte Carlo com Amostragem por Importância, na técnica de Amostragem Assintótica e na técnica de Amostragem Melhorada. Nestes gráficos são adotados 100 pontos para a análise da convergência.

a) Média

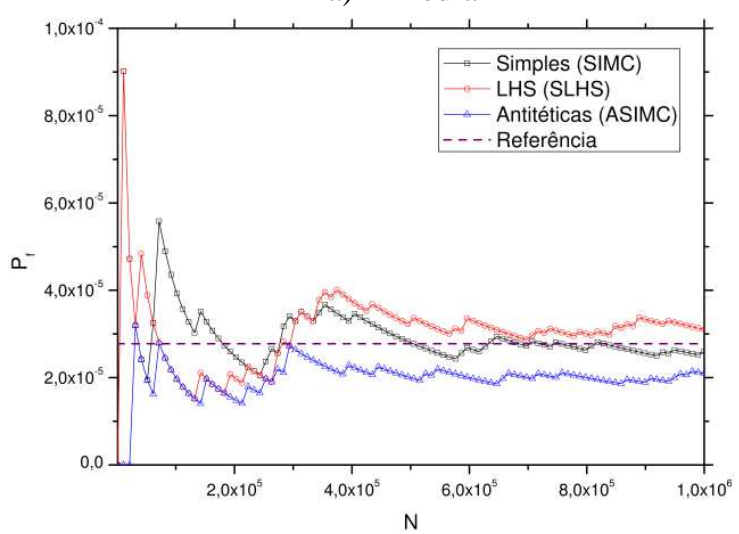

b) C.V.

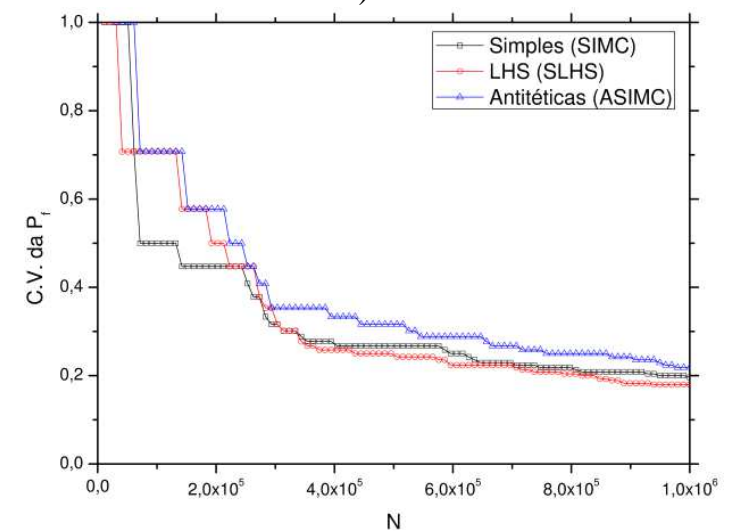

Figura 81. Comparativo da convergência da $P_{f}($ a) e de C.V. (b) para o Monte Carlo Bruto, no Exemplo 4.

a) Média

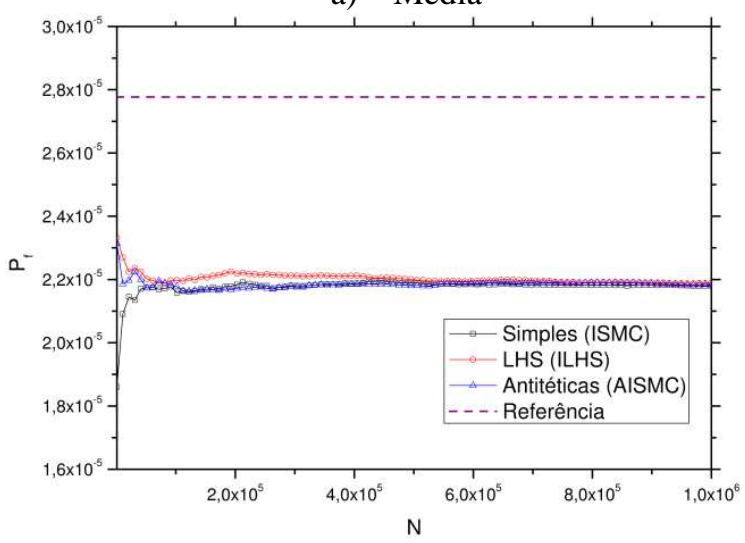

b) C.V.

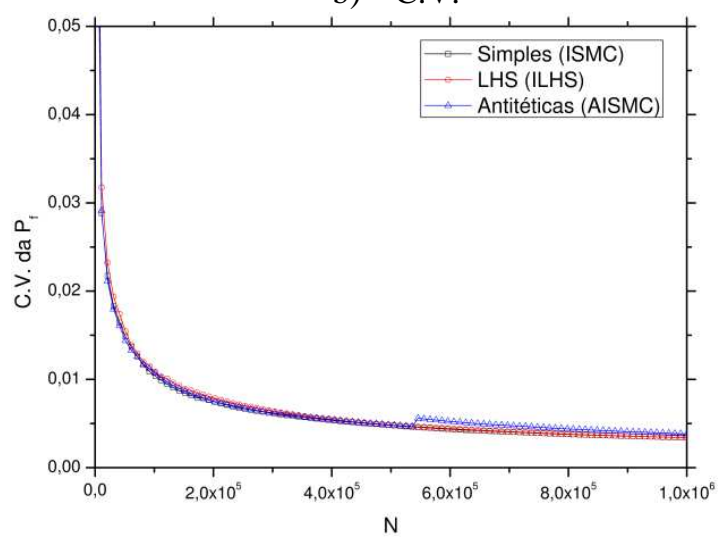

Figura 82. Comparativo da convergência da $P_{f}($ a) e de C.V. (b) para a Amostragem por Importância, no Exemplo 4. 
a) Média

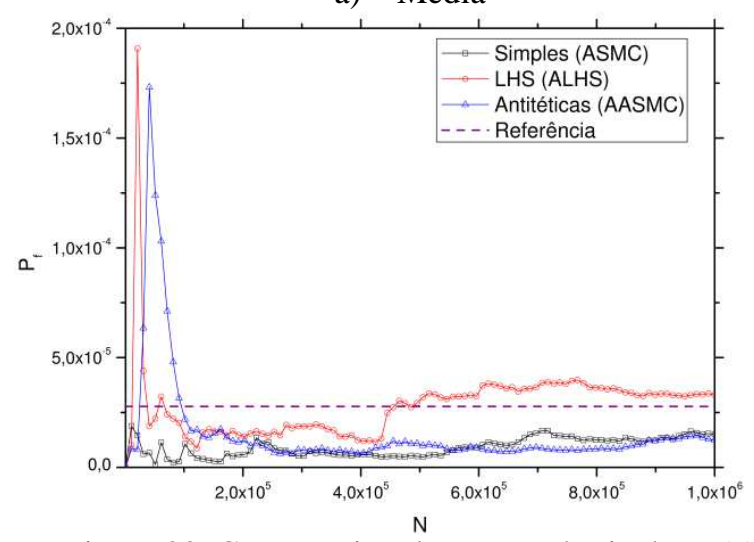

b) C.V.

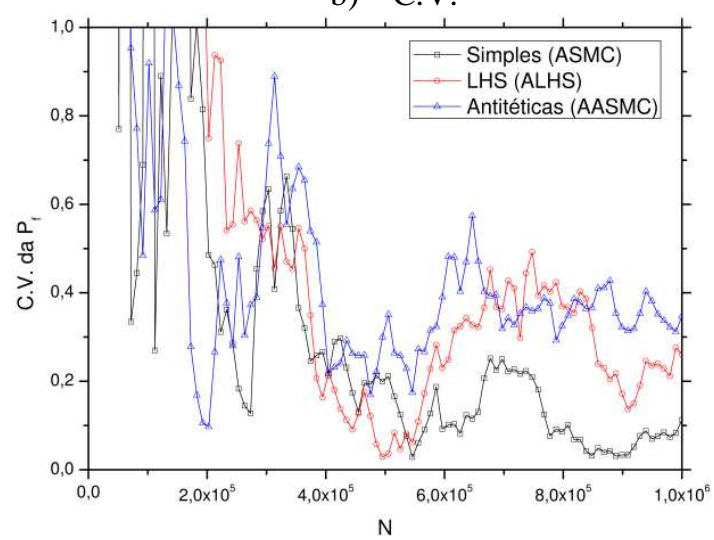

Figura 83. Comparativo da convergência da $P_{f}$ (a) e de C.V. (b) para a Amostragem Assintótica, no Exemplo 4.

a) Média

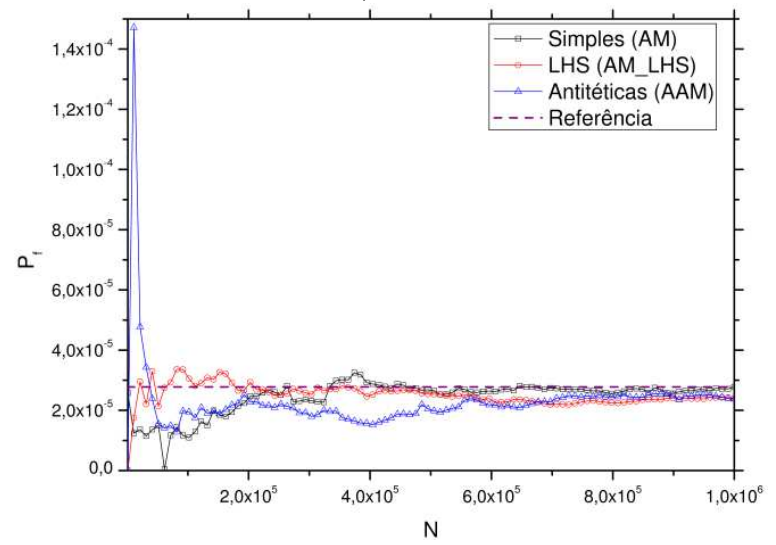

b) C.V.

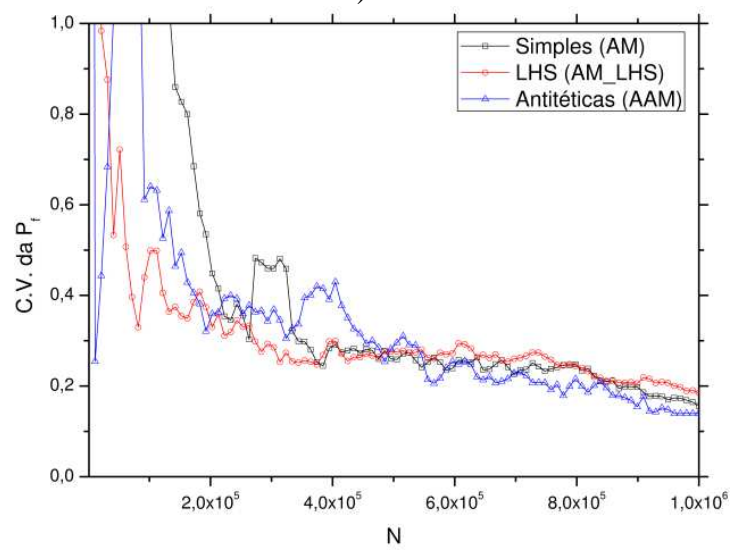

Figura 84. Comparativo da convergência da $P_{f}($ a) e de C.V. (b) para a Amostragem Melhorada, no Exemplo 4.

Na Figura 81a e na Figura 81b não é possível notar vantagem da utilização no Monte Carlo Bruto, da Amostragem por Hipercubo Latino e da Amostragem por Variáveis Antitéticas. Figura 82a, é fácil ver que a Amostragem por Importância, apesar de apresentar uma rápida convergência, leva a um resultado tendencioso, mesmo apresentado um baixo coeficiente de variação da probabilidade de falha (Figura 82b). Na Figura 83a, é fácil perceber a influência da Amostragem por Hipercubo Latino na Amostragem Assintótica, onde a partir de uma amostra de tamanho $4 \times 10^{5}$ há um salto no gráfico de convergência, isso ocorre provavelmente devido ao uso de diferentes distribuições de probabilidade. Além disso, na Figura $83 b$ se observa uma forte flutuação do coeficiente de variação da probabilidade de falha na Amostragem Assintótica, especialmente para amostras pequenas. Mas estudos podem ser realizados para avaliar este fenômeno, mas não é o objetivo deste trabalho. Na Figura 84a, observa-se que a Amostragem por Hipercubo Latino é vantajosa na Amostragem Melhorada, 
pois, se observa uma rápida convergência e um menor coeficiente de variação da probabilidade de falha (Figura $84 \mathrm{~b}$ ) para amostras pequenas.

\subsubsection{Comparativo da probabilidade de falha e de seu coeficiente de variação}

Nesta seção é avaliada a aplicação da técnica de Simulação de Subconjuntos e também será realizado um comparativo da resposta desta técnica com as outras técnicas aqui estudadas. Considera-se uma amostra de tamanho 2.300. Tais resultados podem ser observados na Tabela 26. Na Simulação de Subconjuntos, adota-se $\alpha=1,5$, caminhante aleatório com distribuição uniforme de probabilidade e $P_{0}=0,1$, sendo o tamanho de cada subconjunto igual a 500 .

Tabela 26. Comparativo da $P_{f}$ para uma amostra de tamanho 2.300, no Exemplo 4.

\begin{tabular}{|c|c|c|c|c|}
\hline \multicolumn{5}{|c|}{$P_{f}$ de referência: $2,777 \times 10^{-5}$} \\
\hline \multicolumn{5}{|c|}{ SIMPLES } \\
\hline Sigla & Técnica & $\boldsymbol{P}_{\boldsymbol{f}}$ & C.V.da $P_{f}$ & Erro $(\%)$ \\
\hline BRUTO & Bruto & -- & -- & -- \\
\hline AI & Importância & $1,973 \times 10^{-5}$ & 0,0551 & 28,9521 \\
\hline $\mathbf{A A}$ & Assintótica & -- & -- & -- \\
\hline $\mathbf{A M}$ & Melhorada & $1,152 \times 10^{-4}$ & 0,5941 & 314,8362 \\
\hline SS & Subconjuntos & $2,640 \times 10^{-5}$ & 0,4741 & 4,9334 \\
\hline \multicolumn{5}{|c|}{ HIPERCUBO LATINO } \\
\hline Sigla & Técnica & $P_{f}$ & C.V.da $P_{f}$ & Erro (\%) \\
\hline BRUTO & Bruto & -- & -- & -- \\
\hline AI & Importância & $1,947 \times 10^{-5}$ & 0,0587 & 29,8884 \\
\hline $\mathbf{A A}$ & Assintótica & -- & -- & -- \\
\hline $\mathbf{A M}$ & Melhorada & $5,129 \times 10^{-7}$ & 144,2610 & 98,153 \\
\hline SS & Subconjuntos & $1,670 \times 10^{-5}$ & 0,5101 & 39,8632 \\
\hline \multicolumn{5}{|c|}{ VARIÁVEIS ANTITÉTICAS } \\
\hline Sigla & Técnica & $\boldsymbol{P}_{f}$ & C.V.da $P_{f}$ & Erro $(\%)$ \\
\hline BRUTO & Bruto & -- & -- & -- \\
\hline AI & Importância & $2,258 \times 10^{-5}$ & 0,0661 & 18,6892 \\
\hline $\mathbf{A A}$ & Assintótica & -- & -- & -- \\
\hline $\mathbf{A M}$ & Melhorada & $1,369 \times 10^{-5}$ & 4,1940 & 50,7022 \\
\hline SS & Subconjuntos & $1,078 \times 10^{-5}$ & 0,5559 & 61,1811 \\
\hline
\end{tabular}

O resultado da Tabela 26 é ilustrado graficamente na Figura 85. Neste caso se observa que o Monte Carlo Bruto não apresentou resultados para uma amostra deste tamanho. Por outro lado, o método de Monte Carlo com Amostragem por Importância apresenta uma redução significativa do coeficiente de variação da probabilidade de falha, como era esperado. É observado que a técnica de Simulação de Subconjuntos apresenta um bom resultado em termos do valor médio e do coeficiente de variação da probabilidade de falha. 


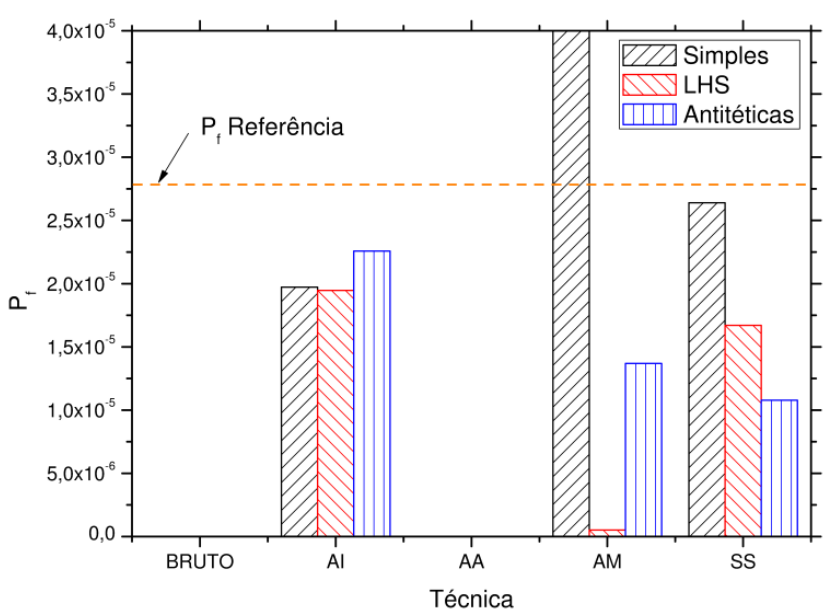

Figura 85. Comparativo da probabilidade de falha para uma amostra de tamanho 2.300, no Exemplo 4.

\subsubsection{Tempo de processamento}

Nesta seção são apresentados os gráficos da relação entre o tempo de processamento e o aumento do tamanho da amostra. Na Figura 86 e na Figura 87, comparam-se os tempos de processamento do Monte Carlo Bruto (BRUTO), do Monte Carlo com Amostragem por Importância (AI), da Amostragem Assintótica (AA) e da Amostragem Melhorada (AM). É feito o uso da Amostragem Simples, da Amostragem por Hipercubo Latino e da Amostragem por Variáveis Antitéticas, como técnicas de amostragem básica. O tamanho da amostra varia de $1 \times 10^{5}$ até $9 \times 10^{5}$. Já na Figura 88 a se compara os tempos de processamento da técnica de Simulação de Subconjuntos (SS) e na Figura 88b se compara os tempos de processamento de todas as técnicas estudadas neste trabalho. Na Figura 88, o tamanho da amostra varia de 9.200 até 46.000. Para a Simulação de Subconjuntos, consideram-se os mesmos parâmetros utilizados no estudo comparativo apresentado em 7.4.4, com o tamanho de cada subconjunto variando de 2.000 até 10.000 , com um intervalo de 2.000 entre cada tamanho de subconjunto. 
a) Monte Carlo Bruto

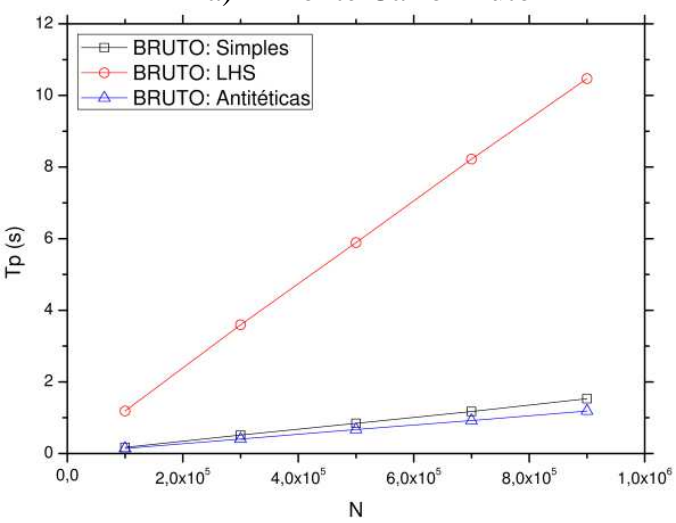

b) Amostragem por Importância

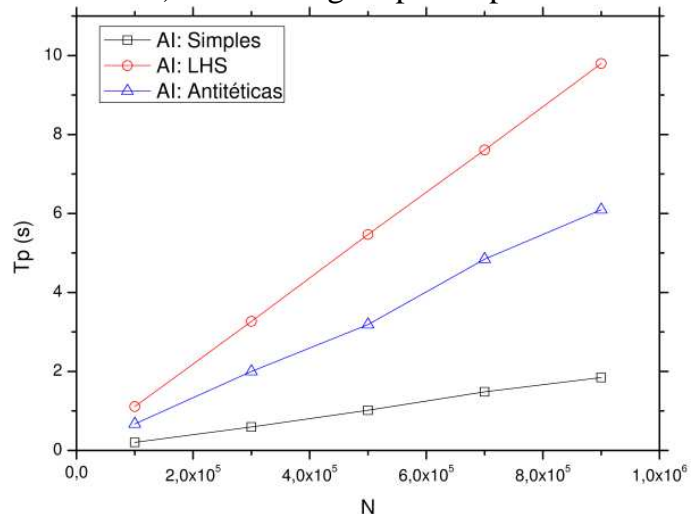

Figura 86. Relação entre o tempo de processamento e o tamanho da amostra no Exemplo 4: Monte Carlo Bruto (a) e Amostragem por Importância (b).

a) Amostragem Assintótica

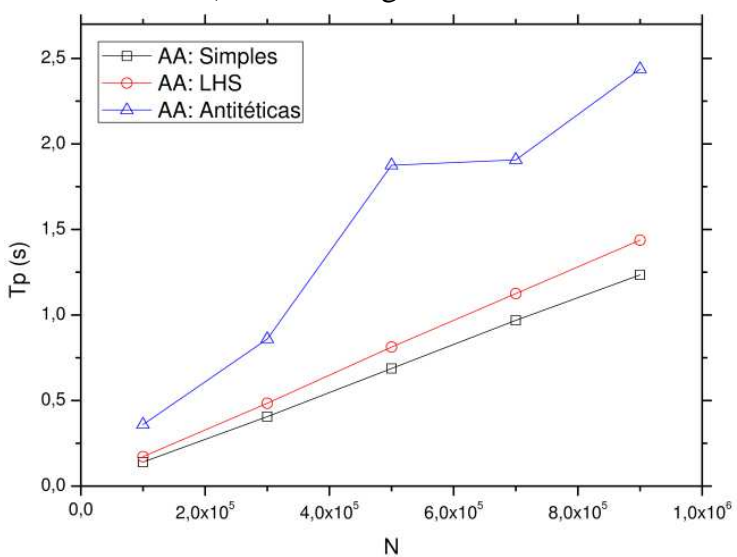

b) Amostragem Melhorada

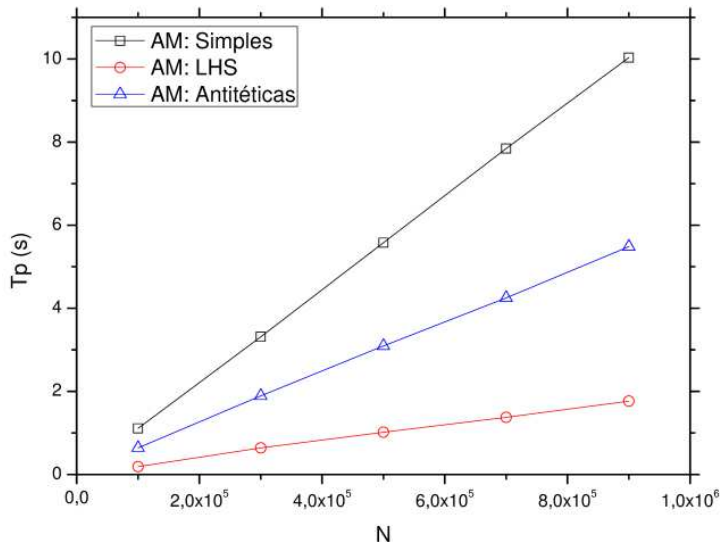

Figura 87. Relação entre o tempo de processamento e o tamanho da amostra no Exemplo 4: Amostragem Assintótica (a) e Amostragem Melhorada (b).

a) Simulação de Subconjunto

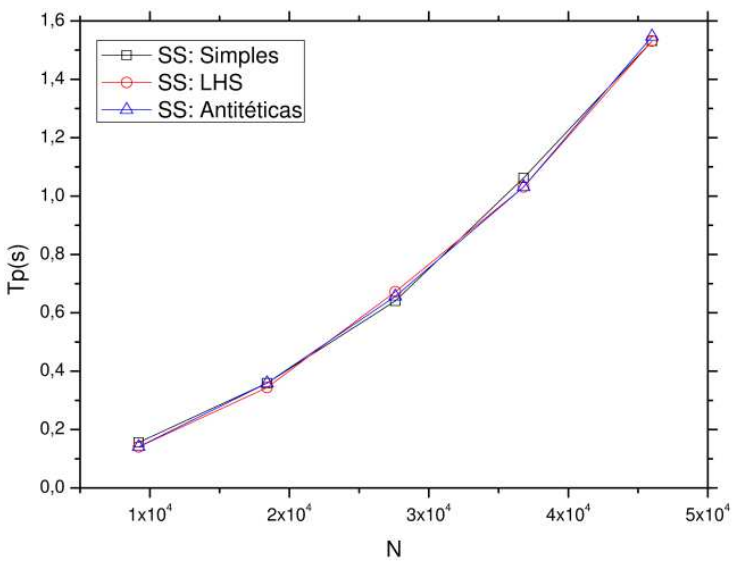

b) Todas as técnicas

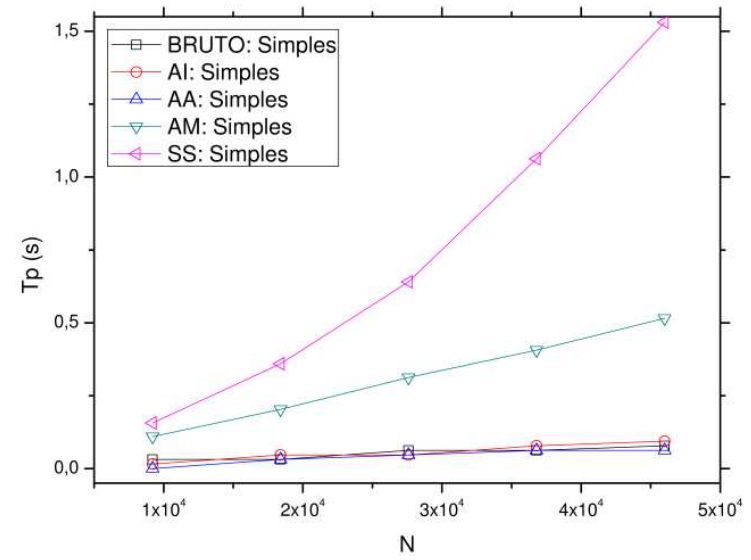

Figura 88. Relação entre o tempo de processamento e o tamanho da amostra no Exemplo 4: Simulação de Subconjuntos (a) e comparativo entre todas as técnicas(b).

De início se observa na Figura 86a que devido ao maior número de variáveis aleatórias, o tempo de processamento da simulação do Monte Carlo Bruto com Amostragem por 
Hipercubo Latino aumenta. Tal comportamento é observado também na Amostragem por Importância (Figura 86b), onde o tempo de processamento utilizando a Amostragem por Variáveis Antitéticas também aumenta. Na Figura 87a, que é a análise referente a Amostragem Assintótica, se observa apenas um aumento significativo do tempo de processamento quando se utiliza a Amostragem por Variáveis Antitéticas. Já na Amostragem Melhorada (Figura 87b), a utilização da Amostragem Simples leva a um aumento do tempo de processamento em relação a Amostragem por Hipercubo Latino e a Amostragem por Variáveis Antitéticas.

Na Figura 88a, nota-se o comportamento não linear, tal como visto nos problemas anteriores, para a relação entre o tempo de processamento e o tamanho da amostra, na Simulação de Subconjuntos. Na Figura 88 b é realizado um comparativo entre todas as técnicas para os mesmos tamanhos de amostra. Dessa forma, se observa que a Simulação de Subconjuntos apresenta o maior tempo de processamento, seguido pela a Amostragem Melhorada. Porém, a Simulação de Subconjuntos leva a uma boa estimativa da probabilidade de falha com uma amostra pequena, onde o tempo de processamento é baixo para todas as técnicas.

\subsection{Exemplo 5: Treliça Hiperestática}

Este exemplo é uma adaptação do problema apresentado por Verzenhassi (2008) e procura analisar a probabilidade de falha de um sistema estrutural. É realizado o estudo em uma treliça hiperestática formada por perfis de aço. As propriedades geométricas dos perfis são apresentadas na Tabela 27. A estrutura estudada é ilustrada na Figura 89.

Tabela 27. Propriedades dos perfis de aço utilizados no Exemplo 5 (Verzenhassi, 2008).

\begin{tabular}{|c|c|c|c|}
\hline Barra & Perfil U & $\mathrm{A}\left(\mathrm{cm}^{2}\right)$ & $I_{y}\left(\mathrm{~cm}^{4}\right)$ \\
\hline 1 & U 50x $25 \times 2,00$ & 1,87 & 1,13 \\
\hline 2 & U 50x $25 \times 2,00$ & 1,87 & 1,13 \\
\hline 3 & U 50x $25 \times 2,00$ & 1,87 & 1,13 \\
\hline 4 & U $50 \times 25 \times 2,00$ & 1,87 & 1,13 \\
\hline 5 & U $50 \times 25 \times 2,00$ & 1,87 & 1,13 \\
\hline 6 & U $75 \times 40 \times 1,20$ & 1,81 & 2,97 \\
\hline
\end{tabular}




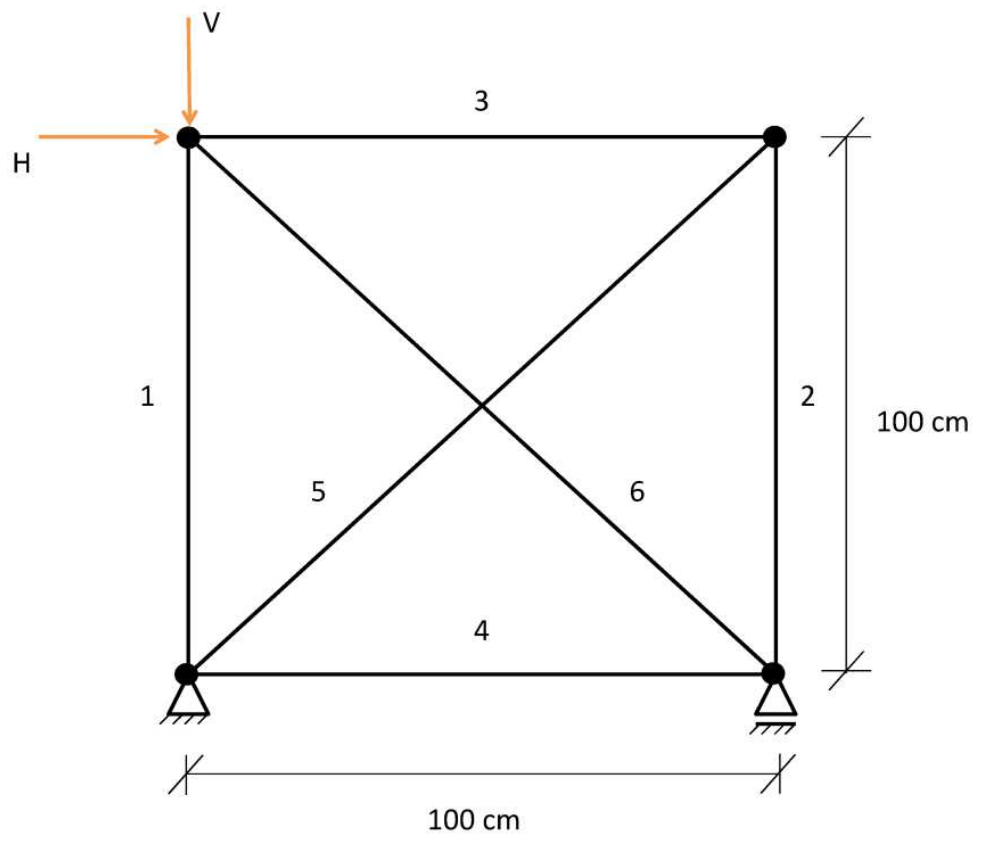

Figura 89.Ilustração da treliça hiperestática estudada.

A princípio podem-se definir duas equações de estado limite para cada barra, sendo uma referente à flambagem elástica (Eq. (143)) e outra referente ao escoamento da seção (Eq. (144)), considerando o comportamento de treliça ideal.

$$
\begin{gathered}
g_{i}\left(E_{i}, L_{i}, V, H\right)=\frac{\pi^{2} E_{i} I_{i}}{L_{i}^{2}}+\left(a_{i} V+b_{i} H\right), \operatorname{com} i=1, \ldots, 6, \\
g_{2}\left(A_{i}, f_{y_{i}}, V, H\right)=A_{i} \cdot f_{y_{i}}-\left(a_{i} V+b_{i} H\right), \operatorname{com} i=1, \ldots, 6,
\end{gathered}
$$

onde $E_{i}$ é o módulo de elasticidade, $I_{i}$ é o momento de inércia da seção do perfil $\mathrm{U}, L_{i}$ é o comprimento de cada perfil $\mathrm{U}, A_{i}$ é a área da seção transversal do perfil $\mathrm{U}, f_{y_{i}}$ é a tensão de escoamento do aço, $V$ é o carregamento vertical, $H$ é o carregamento horizontal, $a_{i}$ é a parcela da carga vertical que é transferida para cada barra, $b_{i}$ é a parcela da carga horizontal que é transferida para cada barra.

Além disso, poderia ser considerada uma árvore de falhas, de forma a definir a falha a partir do momento que a treliça passa a ser considerada hipostática. Porém, neste trabalho a falha será caracterizada pela falha de qualquer componente da treliça, de forma que isso inviabilize seu uso e exija a troca da peça. 
São consideradas quatro variáveis aleatórias, cujos parâmetros são definidos na Tabela 28. Além disso, se considera uma correlação de 0,1 entre os carregamentos horizontal $(H)$ e vertical $(V)$.

Tabela 28. Médias e coeficientes de variação das variáveis aleatórias do Exemplo 5.

\begin{tabular}{lcccc}
\hline Variável Aleatória & Distribuição & Média & C.V. & Unidade \\
\hline Módulo de Elasticidade $(\boldsymbol{E})$ & Log-normal & 20.500 & 0,05 & $\mathrm{kN} / \mathrm{cm}^{2}$ \\
Tensão de escoamento $\left(\boldsymbol{f}_{\boldsymbol{y}}\right)$ & Log-normal & 25 & 0,05 & $\mathrm{kN} / \mathrm{cm}^{2}$ \\
Carregamento Vertical $(\boldsymbol{V})$ & Log-normal & 10 & 0,2 & $\mathrm{kN}$ \\
Carregamento Horizontal $(\boldsymbol{H})$ & Log-normal & 10 & 0,3 & $\mathrm{kN}$ \\
\hline
\end{tabular}

Os estados limites adotados são os de flambagem elástica nas barras 1, 2, 3 e 6; e escoamento das barras 4 e 5. Inicialmente se utiliza o método de Monte Carlo Bruto com a Amostragem Simples, para definir o valor da probabilidade de falha de referência. Neste caso, é utilizada uma amostra de tamanho $2 \times 10^{9}$. Assim, foi possível obter uma probabilidade de falha de $1,139 \times 10^{-4}(\beta=3,686)$, com o coeficiente de variação de 0,0022. Esta simulação durou $28 \min$ e $56 \mathrm{~s}$.

A seguir são apresentados os estudos referentes à aplicação das técnicas estudadas neste trabalho. Inicialmente é apresentado um estudo relativo à Amostragem por Importância.

\subsubsection{Amostragem por Importância}

A Amostragem por Importância utilizando os pontos de projeto requer o conhecimento prévio dos $n$ pontos de projeto, onde $n$ é o número de equações de estado limite que compõe o sistema. Como neste problema são adotadas seis equações de estado limite, então seis pontos de projetos são inicialmente definidos como pontos onde as funções de amostragem são centradas, porém, os estados limites 4 e 5 são desconsiderados, pois a importância deles, na estimativa da probabilidade de falha, é pequena.

Uma dificuldade enfrentada é a geração de gráficos de convergência, uma vez que no StRAnD, as amostras são geradas para cada função de amostragem, assim, o gráfico de convergência apresenta um comportamento progressivo. De acordo com a Figura 90, para o estado limite de maior importância, uma quantidade maior de pontos amostrais é utilizada. Esse valor vai diminuindo à medida que diminui a importância dos outros estados limites. Porém, vale destacar que esse comportamento prejudica somente a análise da convergência, pois, o resultado acumulado da Amostragem por Importância para cada estado limite tende a resultar em uma boa estimativa da probabilidade de falha. Portanto, a amostragem por importância não será comparada com as outras técnicas por meio de gráficos de convergência, 
mas sim pelo resultado final da simulação. Aprimoramentos deverão ser incorporados ao StRAnD de maneira a melhorar a apresentação deste tipo de resultado.

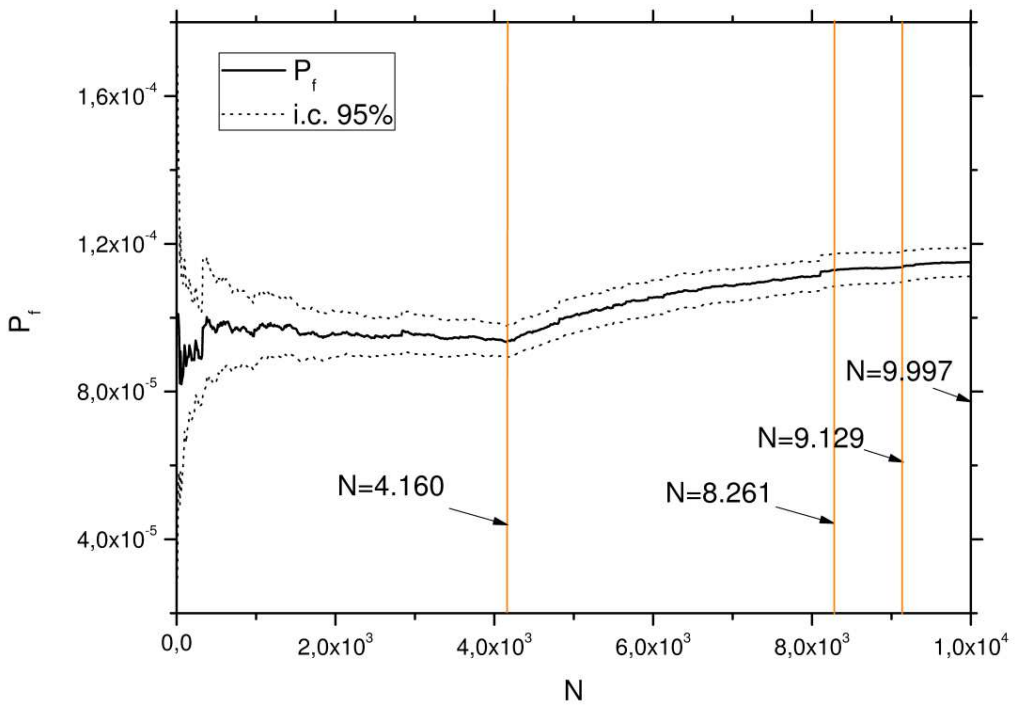

Figura 90. Convergência para a Amostragem por Importância, no Exemplo 5.

\subsubsection{Amostragem Assintótica}

Nesta seção é realizado um estudo da aplicação da Amostragem Assintótica na resolução de um problema que envolve análise de sistemas em confiabilidade de estruturas. Tal estudo é realizado por meio de gráficos de regressão para amostras de tamanho $1 \times 10^{3}$ (Figura 91) e $1 \times 10^{5}$ (Figura 92). Utilizam-se a Amostragem Simples, a Amostragem por Hipercubo Latino e a Amostragem por Variáveis Antitéticas. O parâmetro $f$ foi variado de 0,5 a 0,8 , onde utilizam-se 5 pontos de suporte.

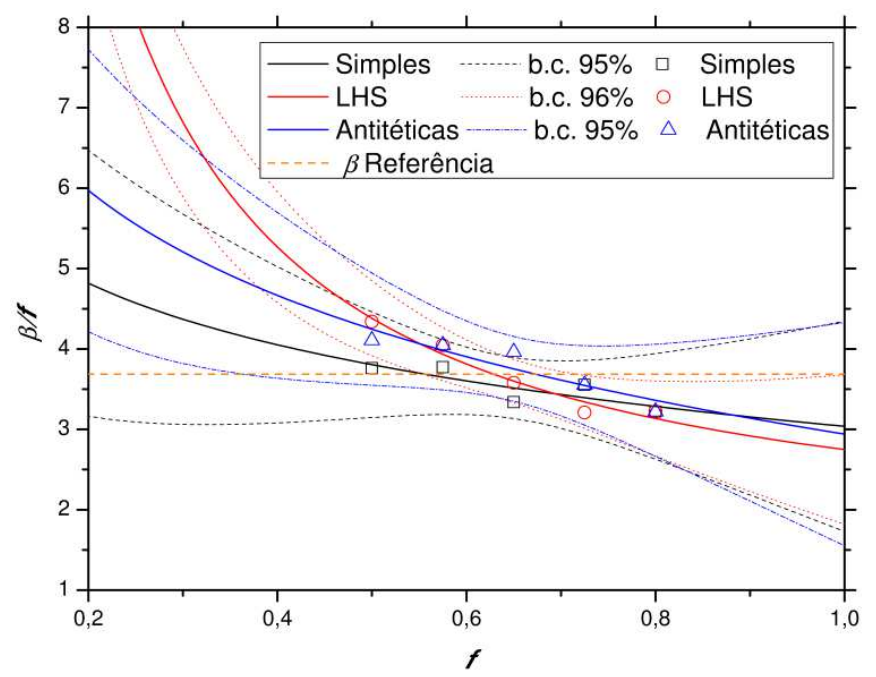

Figura 91. Regressão não linear utilizada na Amostragem Assintótica para amostras de tamanho $1 \times 10^{3}$, no Exemplo 5. 


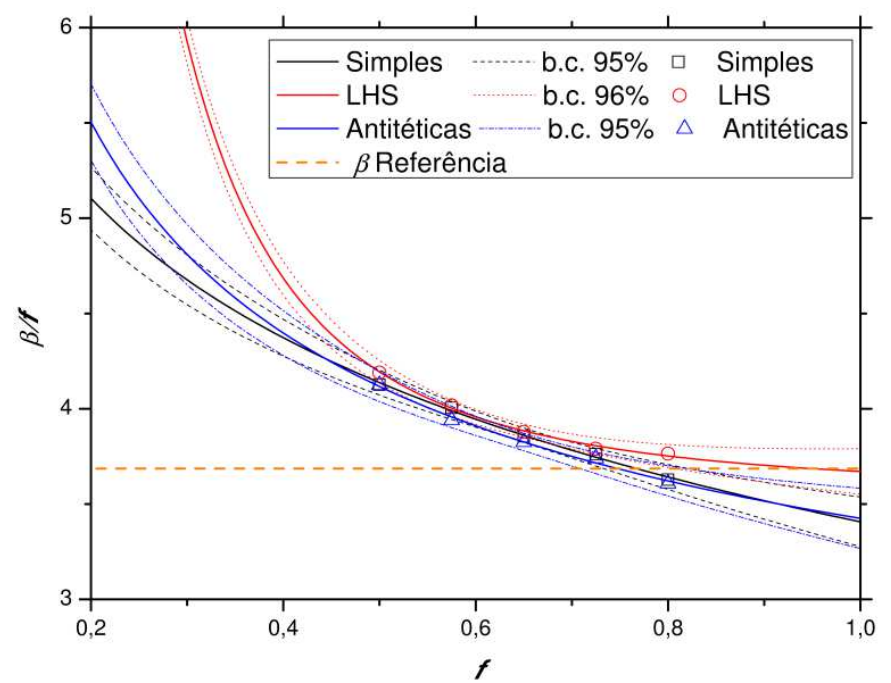

Figura 92. Regressão não linear utilizada na Amostragem Assintótica para amostras de tamanho $1 \times 10^{5}$, no Exemplo 5.

Os parâmetros ajustados na técnica em questão, com a utilização da Amostragem Simples (ASMC), da Amostragem por Hipercubo Latino (ALHS) e da Amostragem por Variáveis Antitéticas (AASMC), são apresentados na Tabela 29.

Tabela 29. Parâmetros ajustados na Amostragem Assintótica para o Exemplo 5.

\begin{tabular}{ccccccc}
\hline $\boldsymbol{n}_{\boldsymbol{s}}$ & \multicolumn{1}{c}{$\mathbf{1 \times 1 0}^{\mathbf{3}}$} & \multicolumn{3}{c}{$\mathbf{1 \times \mathbf { 1 0 } ^ { \mathbf { 5 } }}$} \\
\hline & $\boldsymbol{A}$ & $\boldsymbol{B}$ & $\boldsymbol{C}$ & $\boldsymbol{A}$ & $\boldsymbol{B}$ & $\boldsymbol{C}$ \\
ASMC & $-4.120,902$ & $4.123,940$ & $-2,682 \times 10^{-4}$ & $-2.484,862$ & $2.488,268$ & $-4,237 \times 10^{-4}$ \\
ALHS & 1,482 & 1,266 & $-1,195$ & 3,567 & 0,104 & $-2,596$ \\
AASMC & $-2.8679,322$ & $28.682,263$ & $-6,564 \times 10^{-5}$ & 1,803 & 1,621 & $-0,513$ \\
\hline
\end{tabular}

Observa-se que realizando um maior número de simulações, a concordância entre os pontos de suporte e a curva ajustada, melhora significativamente. Tal resultado pode ser observado na Figura 91 e na Figura 92. As probabilidades de falha e seus coeficientes de variação, para diferentes tamanhos de amostra e para a aplicação de diferentes técnicas de amostragem básica, são apresentado na Tabela 30.

Tabela 30. Probabilidade de falha e coeficiente de Variação da probabilidade de falha, para a Amostragem Assintótica, no Exemplo 5.

\begin{tabular}{ccccc}
\hline \multicolumn{5}{c}{$\boldsymbol{P}_{f}$ de referência: $\mathbf{1 , 1 3 9 \times \mathbf { 1 0 } ^ { - 4 }}$} \\
\hline $\boldsymbol{N}$ & $\mathbf{1 \times \mathbf { 1 0 } ^ { \mathbf { 3 } }}$ & $\mathrm{P}_{f}$ & $\mathrm{1 \times 10^{ \mathbf {5 } }}$ \\
& $P_{f}$ & $C . V$. &.$V$. \\
ASMC & $1,192 \times 10^{-3}$ & 17,3986 & $3,290 \times 10^{-4}$ & 0,5133 \\
ALHS & $2,997 \times 10^{-3}$ & 5,3516 & $1,208 \times 10^{-4}$ & 0,0441 \\
AASMC & $1,636 \times 10^{-3}$ & 18,2864 & $3,075 \times 10^{-4}$ & 0,4479 \\
\hline
\end{tabular}


Neste caso, o uso da Amostragem por Hipercubo Latino na Amostragem Assintótica, apresenta-se como o mais vantajoso meio para estimar a probabilidade de falha no problema em questão.

\subsubsection{Amostragem Melhorada}

Nesta seção é realizado o estudo da Amostragem Melhorada. Consideram-se duas amostras, uma de tamanho $1 \times 10^{3}$ e outra de tamanho $1 \times 10^{5}$. Foram utilizados 100 pontos de suporte, com o parâmetro $\lambda$ variando de 0,4 até 0,9 . Onde se observa claramente que a concordância entre a curva ajustada e os pontos de suporte, melhora significativamente com o aumento do tamanho da amostra.

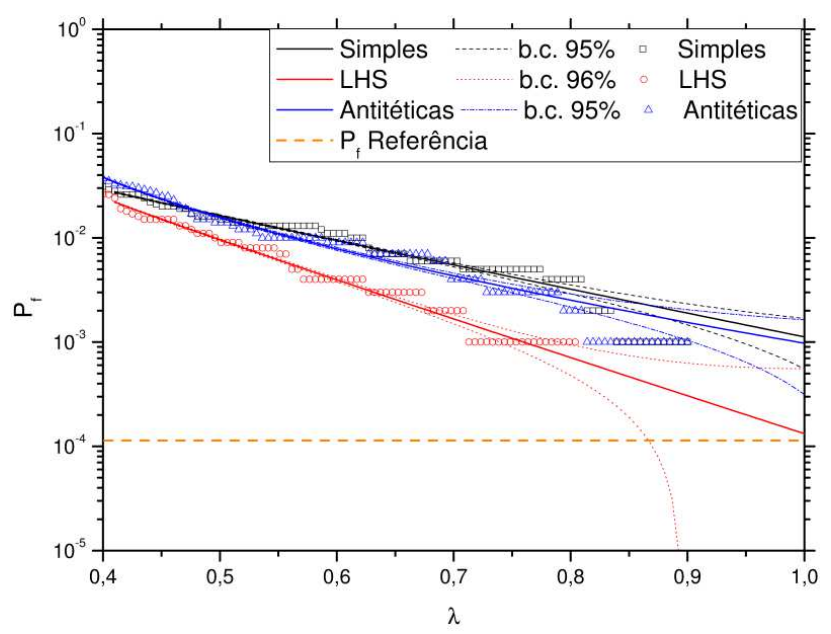

Figura 93. Regressão não linear utilizada na Amostragem Melhorada para amostras de tamanho $1 \times 10^{3}$, no Exemplo 5.

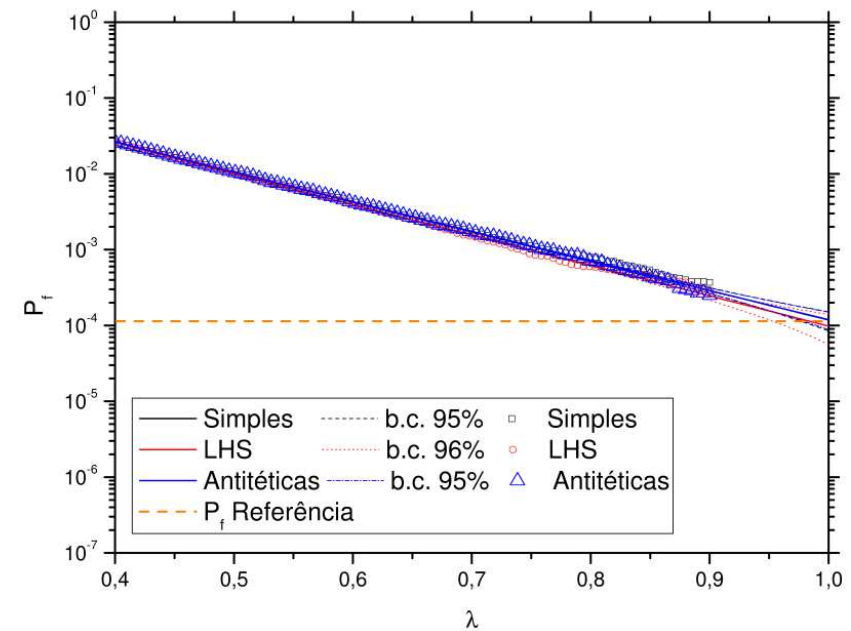

Figura 94. Regressão não linear utilizada na Amostragem Melhorada para amostras de tamanho $1 \times 10^{5}$, no Exemplo 5. 
Os parâmetros ajustados na técnica em questão, utilizando a Amostragem Simples (AM), a Amostragem por Hipercubo Latino (AM_LHS) e a Amostragem por Variáveis Antitéticas (AAM), são apresentados na Tabela 31, para uma amostra de tamanho $1 \times 10^{3}$ e na Tabela 32, para uma amostra de tamanho $1 \times 10^{5}$.

Tabela 31. Parâmetros ajustados na Amostragem Melhorada, para uma amostra de tamanho $1 \times 10^{3}$, no Exemplo

\begin{tabular}{|c|c|c|c|c|}
\hline & $q$ & $a$ & $\boldsymbol{b}$ & $\boldsymbol{c}$ \\
\hline $\mathbf{A M}$ & $2,9165 \times 10^{-2}$ & 5,3352 & 0,4000 & 0,96511 \\
\hline AM_LHS & $2,4420 \times 10^{-2}$ & 8,5153 & 0,4000 & 0,95894 \\
\hline AAM & 0,14562 & 6,3353 & 0,32419 & 0,60112 \\
\hline
\end{tabular}

Tabela 32. Parâmetros ajustados na Amostragem Melhorada, para uma amostra de tamanho $1 \times 10^{5}$, no Exemplo 5.

\begin{tabular}{ccccc}
\hline & $\boldsymbol{q}$ & $\boldsymbol{a}$ & $\boldsymbol{b}$ & $\boldsymbol{c}$ \\
\hline AM & 0,61922 & 9,0525 & $5,4526 \times 10^{-2}$ & 0,98820 \\
AM_LHS & $2,6022 \times 10^{-2}$ & 9,2385 & 0,4000 & 0,98684 \\
AAM & $3,3193 \times 10^{-2}$ & 8,9463 & 0,37777 & 0,97621 \\
\hline
\end{tabular}

A Tabela 33 apresenta os valores das probabilidades de falha e de seus coeficientes de variação, para diferentes tamanhos de amostra.

Tabela 33. Probabilidade de falha e coeficiente de Variação da probabilidade de falha, para a Amostragem Melhorada, no Exemplo 5.

\begin{tabular}{|c|c|c|c|c|}
\hline \multicolumn{5}{|c|}{$P_{f}$ de referência: $1,139 \times 10^{-4}$} \\
\hline$N$ & & & & \\
\hline & $P_{f}$ & C.V. & $P_{f}$ & $C . V$. \\
\hline $\mathbf{A M}$ & $1,1121 \times 10^{-3}$ & 0,2582 & $1,181 \times 10^{-4}$ & 0,1453 \\
\hline AM_LHS & $1,324 \times 10^{-4}$ & 1,6293 & $9,813 \times 10^{-5}$ & 0,2177 \\
\hline $\mathbf{A} \mathbf{A} M$ & $9,755 \times 10^{-4}$ & 0,3461 & $1,191 \times 10^{-4}$ & 0,1336 \\
\hline
\end{tabular}

Dessa forma, se observa vantagem no uso da Amostragem por Hipercubo Latino em conjunto com a Amostragem Melhorada, na resolução do problema em questão.

\subsubsection{Análise de convergência}

É realizado um comparativo entre o Monte Carlo Bruto, a Amostragem por Importância, a Amostragem Assintótica e a Amostragem Melhorada. Todas as técnicas fazem uso da Amostragem Simples, da Amostragem por Hipercubo Latino e da Amostragem por Variáveis Antitéticas. O tamanho da amostra varia de $1 \times 10^{3}$ até $1 \times 10^{5}$, onde são escolhidos 100 pontos nesse intervalo. 
a) Média

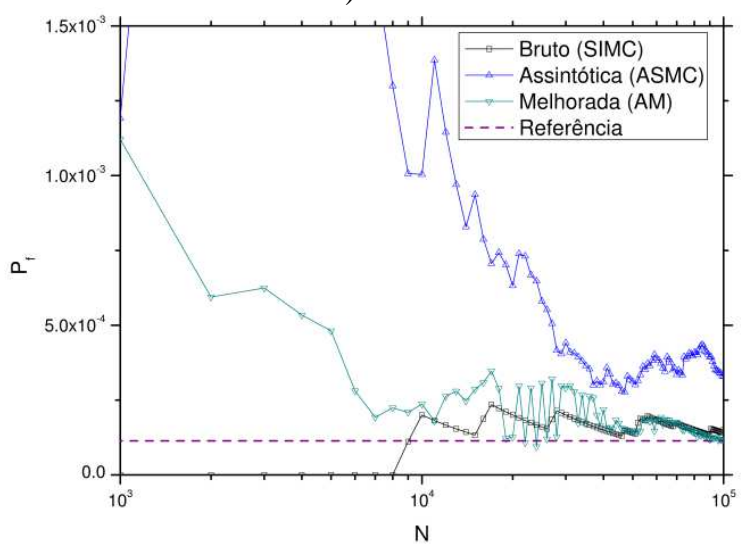

b) C.V.

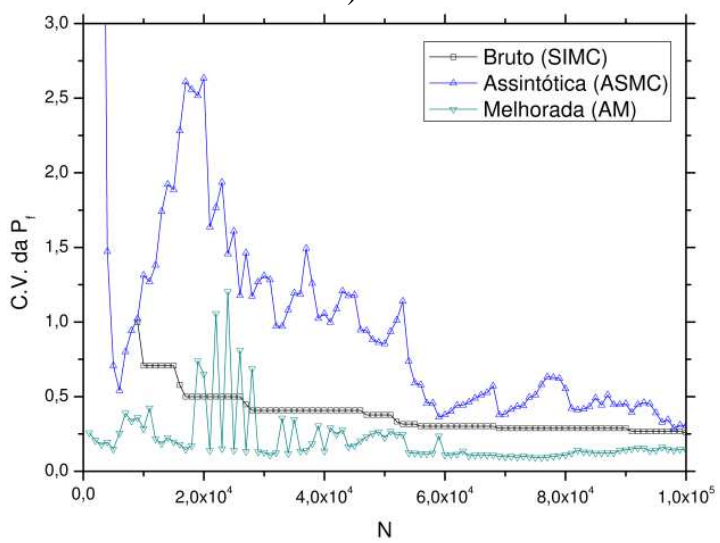

Figura 95. Comparativo da convergência da $P_{f}$ (a) e de C.V. (b) para a Amostragem Simples, no Exemplo 5 .

a) Média

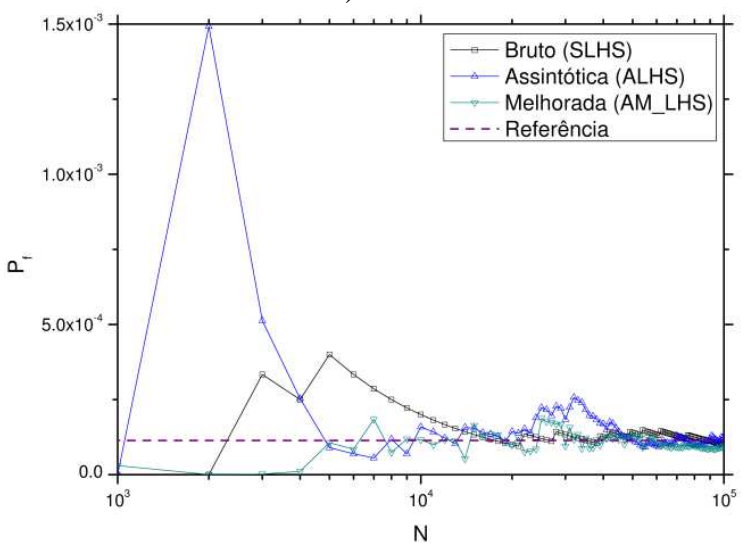

b) C.V.

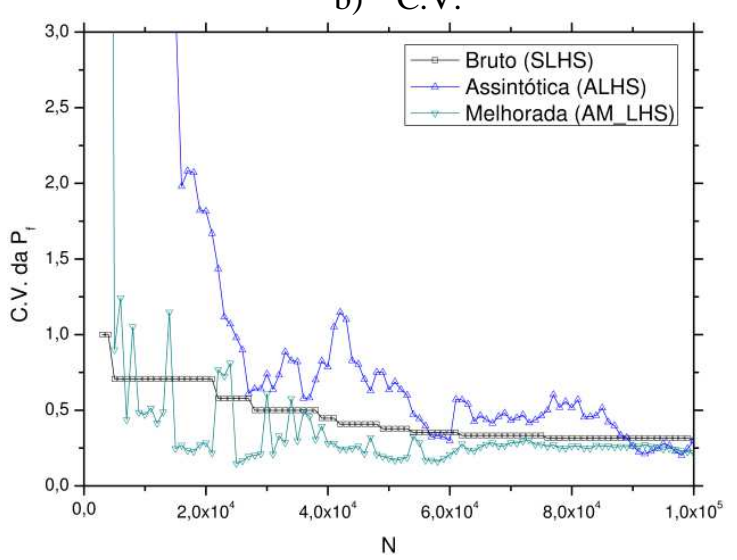

Figura 96. Comparativo da convergência da $P_{f}($ a) e de C.V. (b) para a Amostragem por Hipercubo Latino, no Exemplo 5.

a) Média

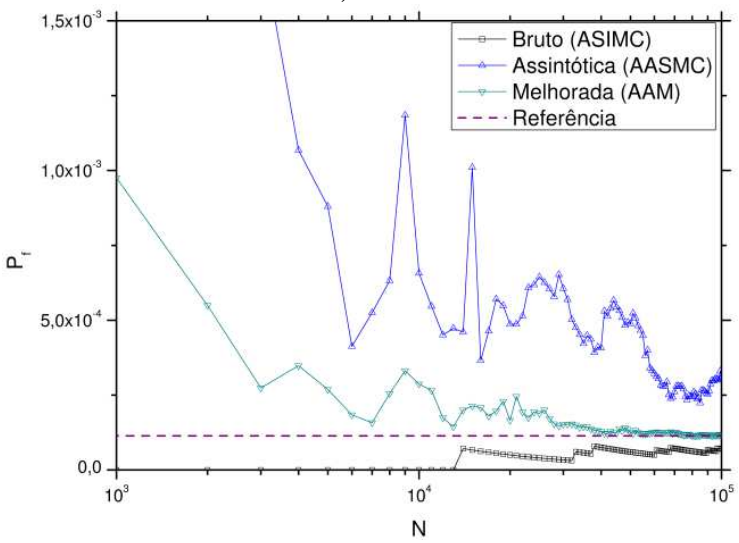

b) C.V.

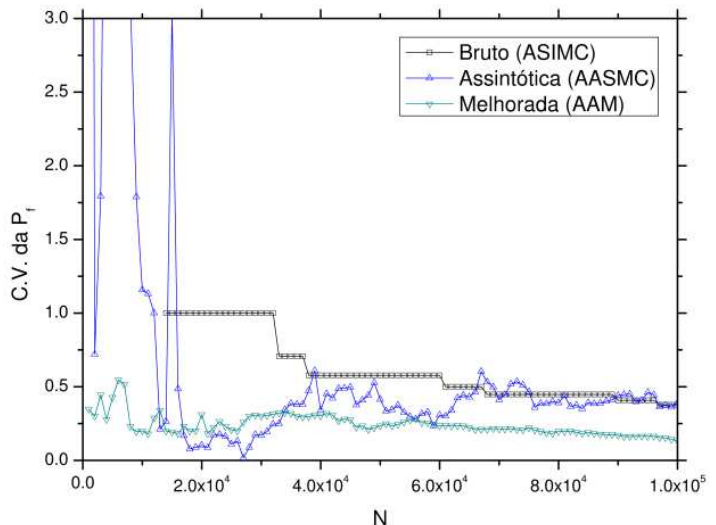

Figura 97. Comparativo da convergência da $P_{f}$ (a) e de C.V. (b) para a Amostragem por Variáveis Antitéticas, no Exemplo 5.

Observa-se na Figura 95a que a técnica de Amostragem Assintótica é a que apresenta um pior resultado, em comparação com o Monte Carlo Bruto e com a Amostragem 
Melhorada. A Amostragem Melhorada apresenta um resultado melhor que o Monte Carlo Bruto. Além disso, observa-se na Figura 95b, que a Amostragem Melhorada apresenta um menor coeficiente de variação, em comparação com as outras técnicas, mesmo apresentando uma certa flutuação nas primeiras simulações. Com a aplicação da Amostragem por Hipercubo Latino, se observa uma boa vantagem da Amostragem Melhorada, em relação à convergência da probabilidade de falha (Figura 96a). Por outro lado, se observa apenas uma leve redução do coeficiente de variação da probabilidade, para a Amostragem Melhorada em relação às outras técnicas (Figura 96b). Na Figura 97a e na Figura 97b, no uso da Amostragem por Variáveis Antitéticas, se precebe a vantagem da Amostragem Melhorada em relação a Amostragem Assintótica e em relação ao Monte Carlo Bruto.

Nos graficos apresentados a seguir, é possível observar a influência da utilização da Amostragem por Hipercubo Latino e Amostragem por Variáveis Antitéticas, em comparação com a Amostragem Simples no Monte Carlo Bruto, no Monte Carlo com Amostragem por Importância, na técnica de Amostragem Assintótica e na técnica de Amostragem Melhorada. Nestes gráficos também são adotados 100 pontos para a análise da convergência.
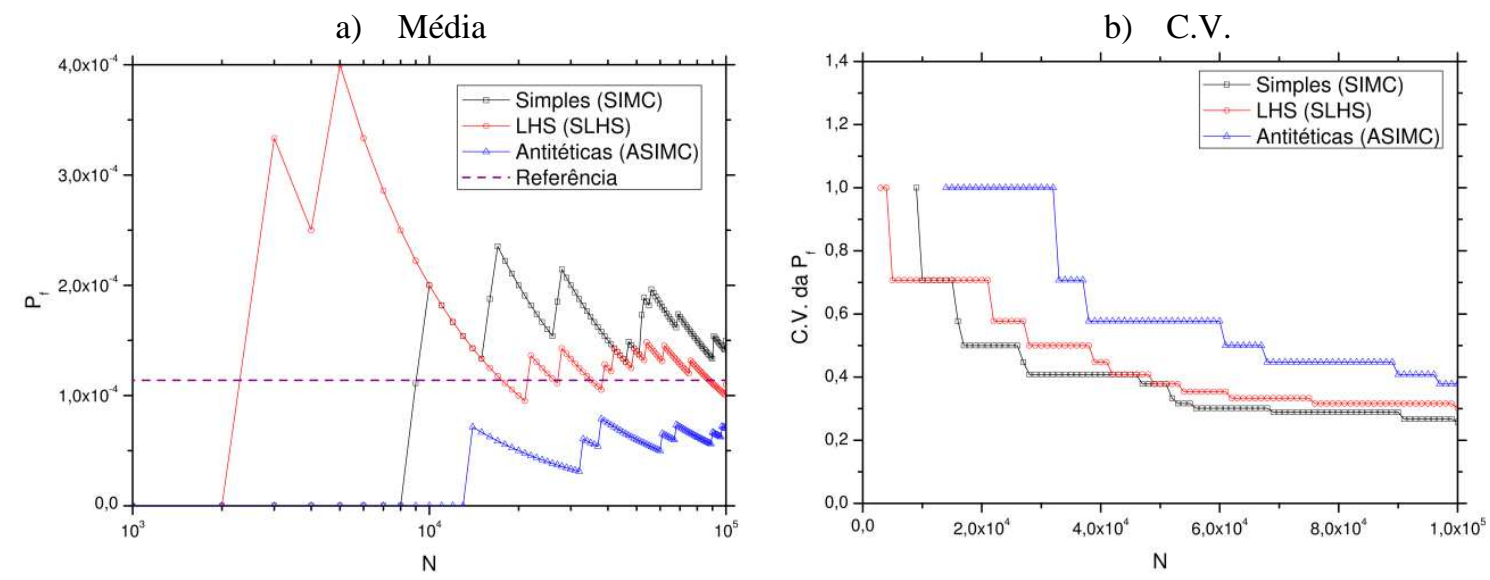

Figura 98. Comparativo da convergência da $P_{f}($ a) e de C.V. (b) para o Monte Carlo Bruto, no Exemplo 5. 
a) Média

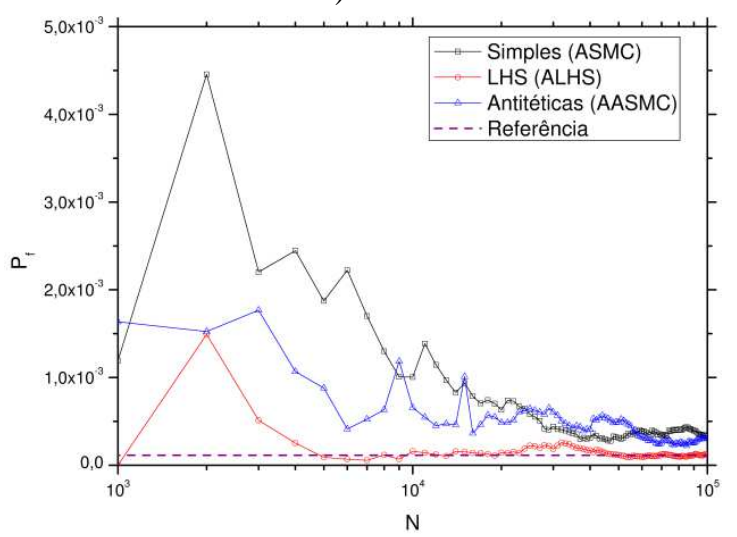

b) C.V.

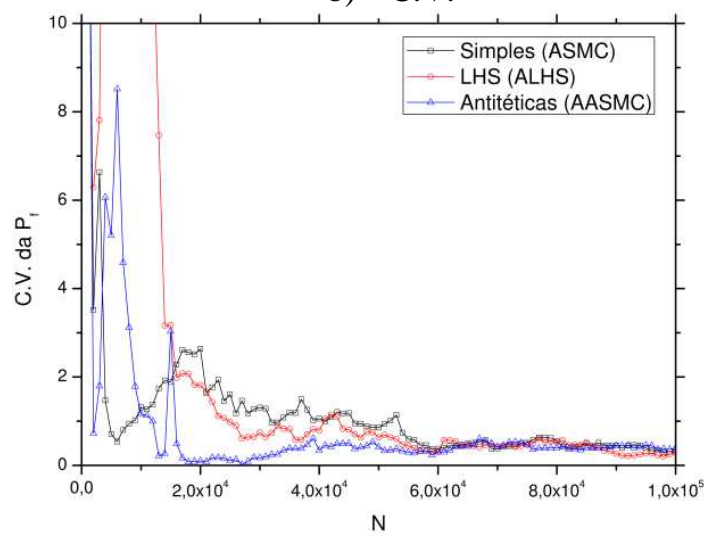

Figura 99. Comparativo da convergência da $P_{f}$ (a) e de C.V. (b) para a Amostragem Assintótica, no Exemplo 5.

a) Média

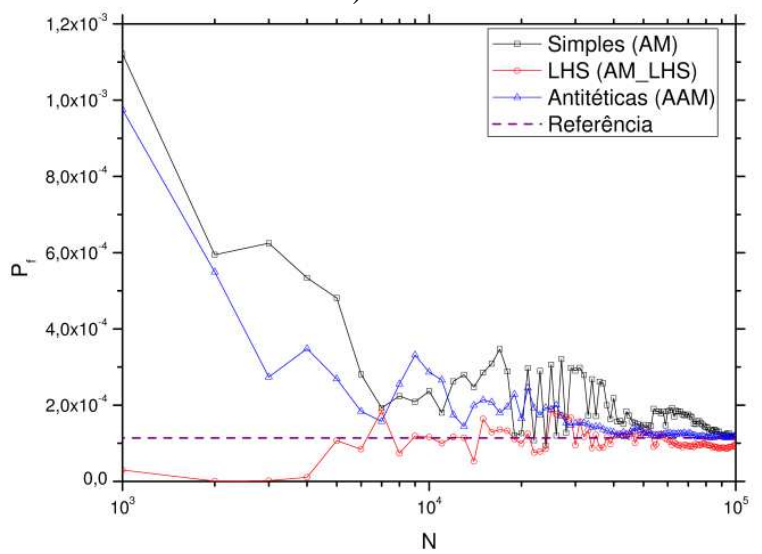

b) C.V.

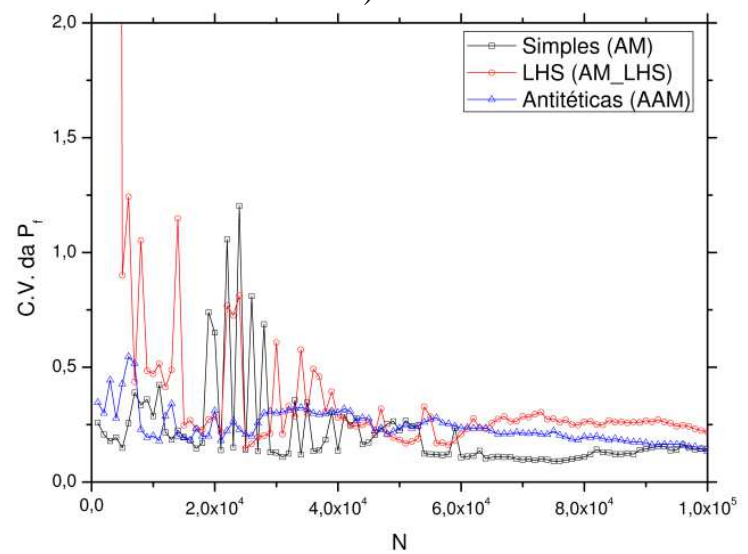

$\mathrm{N}$

Figura 100. Comparativo da convergência da $P_{f}$ (a) e de C.V. (b) para a Amostragem Melhorada, no Exemplo 5.

Nos comparativos realizados na Figura 98, na Figura 99 e na Figura 100, é fácil ver que o uso da Amostagem por Hipercubo Latino é vantajoso em todas as técnicas estudadas, pelo fato de levá-las à uma convergência mais rápida da probabilidade de falha, mesmo que não signifique uma redução do coeficiente de variação da probabilidade de falha. Além disso, a Amostragem por Variáveis Antitéticas só não foi vantajosa quando utilizada em conjunto com o Monte Carlo Bruto (Figura 98a).

\subsubsection{Comparativo da probabilidade de falha e de seu coeficiente de variação}

No estudo comparativo entre as técnicas aqui estudadas é considerada uma amostra de tamanho 3.700. Na técnica de Simulação de Subconjuntos adotam-se os seguintes parâmetros: $N_{S S}=1.000, \alpha=1,5, P_{0}=0,1$. O caminhante aleatório segue uma distribuição de probabilidade uniforme. Tais resultados podem ser observados na Tabela 34 . 
Tabela 34. Comparativo da $P_{f}$ para uma amostra de tamanho 3.700, no Exemplo 5.

\begin{tabular}{|c|c|c|c|c|}
\hline \multicolumn{5}{|c|}{$P_{f}$ de referência: $1,139 \times 10^{-4}$} \\
\hline \multicolumn{5}{|c|}{ SIMPLES } \\
\hline Sigla & Técnica & $P_{f}$ & C.V.da $P_{f}$ & $\operatorname{Erro}(\%)$ \\
\hline BRUTO & Bruto & -- & -- & -- \\
\hline AI & Importância & $1,201 \times 10^{-4}$ & 0,0287 & 5,4434 \\
\hline $\mathbf{A A}$ & Assintótica & $2,382 \times 10^{-3}$ & 1,2903 & 1991,3082 \\
\hline $\mathbf{A M}$ & Melhorada & $6,209 \times 10^{-4}$ & 0,1657 & 445,1273 \\
\hline SS & Subconjuntos & $1,590 \times 10^{-4}$ & 0,3221 & 39,5961 \\
\hline \multicolumn{5}{|c|}{ HIPERCUBO LATINO } \\
\hline Sigla & Técnica & $P_{f}$ & C.V.da $P_{f}$ & $\operatorname{Erro}(\%)$ \\
\hline BRUTO & Bruto & -- & -- & -- \\
\hline AI & Importância & $1,111 \times 10^{-4}$ & 0,0260 & 2,4583 \\
\hline $\mathbf{A A}$ & Assintótica & $3,879 \times 10^{-4}$ & 10,4964 & 240,5619 \\
\hline $\mathbf{A M}$ & Melhorada & $6,830 \times 10^{-5}$ & 1,6205 & 40,0351 \\
\hline SS & Subconjuntos & $1,188 \times 10^{-4}$ & 0,3406 & 4,3020 \\
\hline \multicolumn{5}{|c|}{ VARIÁVEIS ANTITÉTICAS } \\
\hline Sigla & Técnica & $\boldsymbol{P}_{f}$ & C.V.da $P_{f}$ & $\operatorname{Erro}(\%)$ \\
\hline BRUTO & Bruto & -- & -- & -- \\
\hline AI & Importância & $1,171 \times 10^{-4}$ & 0,0292 & 2,8095 \\
\hline $\mathbf{A A}$ & Assintótica & $1,229 \times 10^{-3}$ & 3,1715 & 979,0167 \\
\hline $\mathbf{A M}$ & Melhorada & $3,846 \times 10^{-4}$ & 0,2525 & 237,6646 \\
\hline SS & Subconjuntos & $1,280 \times 10^{-4}$ & 0,3205 & 12,3793 \\
\hline
\end{tabular}

O resultado da Tabela 34 é ilustrado graficamente na Figura 101. Neste caso observa-se que o Monte Carlo Bruto com Amostragem Simples não apresenta resultados para uma amostra deste tamanho. Por outro lado, o método de Monte Carlo com Amostragem por Importância apresenta uma vantagem significativa, como era esperado. É observado que a técnica de Simulação de Subconjuntos apresenta um bom resultado em termos do valor da probabilidade de falha, do coeficiente de variação da probabilidade de falha e do erro relativo. Destaca-se que o uso da Amostragem por Hipercubo Latino e da Amostragem por Variáveis Antitéticas é benéfico para a maioria das técnicas, uma vez que leva a um resultado mais próximo da probabilidade de falha de referência. 


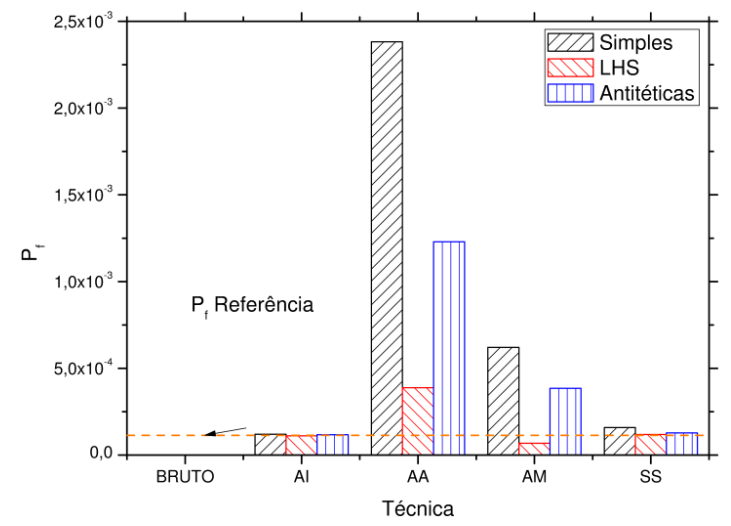

Figura 101. Comparativo da probabilidade de falha para uma amostra de tamanho 3.700, no Exemplo 5.

\subsubsection{Tempo de processamento}

Nesta seção são apresentados os gráficos da relação entre o tempo de processamento e o tamanho da amostra. Na Figura 102 e na Figura 103, comparam-se os tempos de processamento do Monte Carlo Bruto (BRUTO), do Monte Carlo com Amostragem por Importância (AI), da Amostragem Assintótica (AA) e da Amostragem Melhorada (AM). É feito uso da Amostragem Simples, da Amostragem por Hipercubo Latino e da Amostragem por Variáveis Antitéticas, como técnicas de amostragem básica. O tamanho da amostra varia de $1 \times 10^{5}$ até $1,1 \times 10^{6}$. Já na Figura 104 a se compara os tempos de processamento da técnica de Simulação de Subconjuntos (SS) e na Figura 104b se compara os tempos de processamento de todas as técnicas. Na Figura 102 e na Figura 103 o tamanho da amostra varia de 3.700 até 48.100. Para a Simulação de Subconjuntos, consideram-se os mesmos parâmetros utilizados no estudo comparativo apresentado em 7.5.5, com o tamanho de cada subconjunto variando de 1.000 até 11.000 , com um intervalo de 2.000 entre cada tamanho de subconjunto.

a) Monte Carlo Bruto

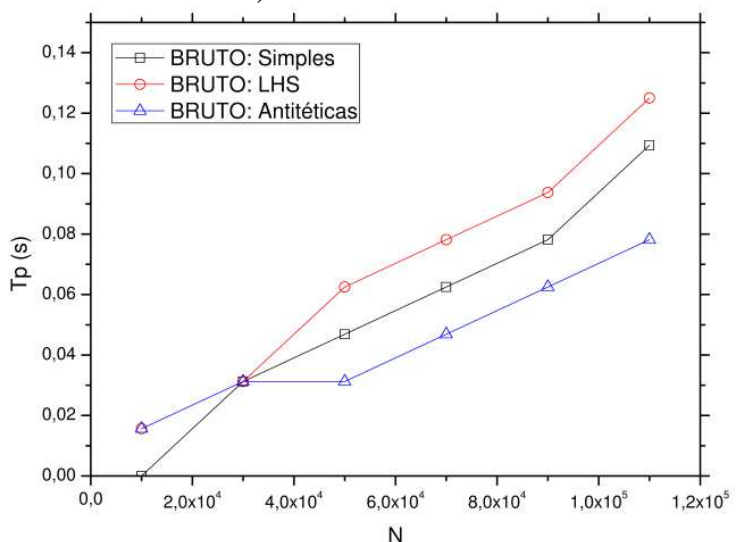

b) Amostragem por Importância

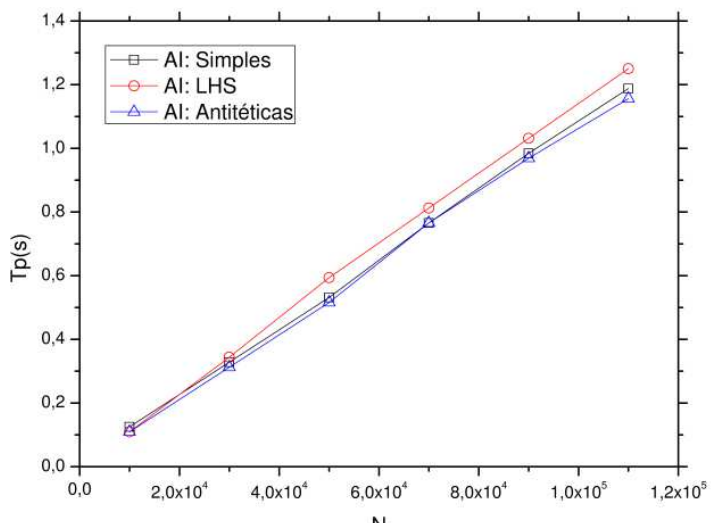

Figura 102. Relação entre o tempo de processamento e o tamanho da amostra no Exemplo 5: Monte Carlo Bruto (a) e Amostragem por Importância (b). 
a) Amostragem Assintótica

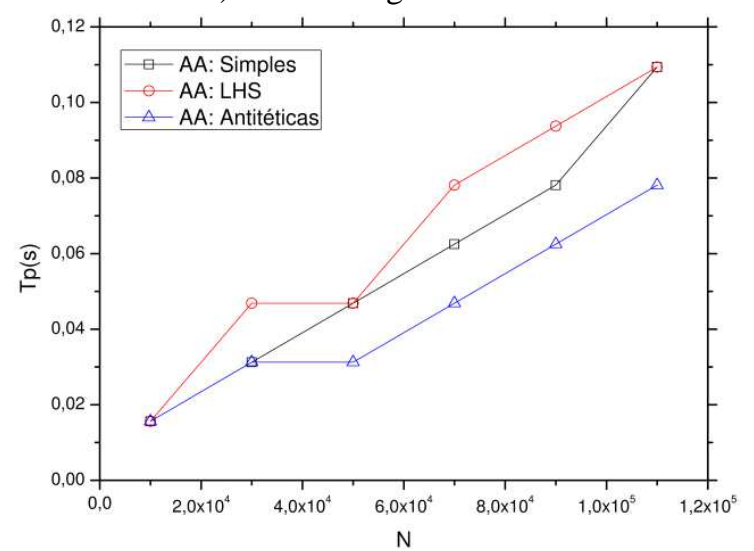

b) Amostragem Melhorada

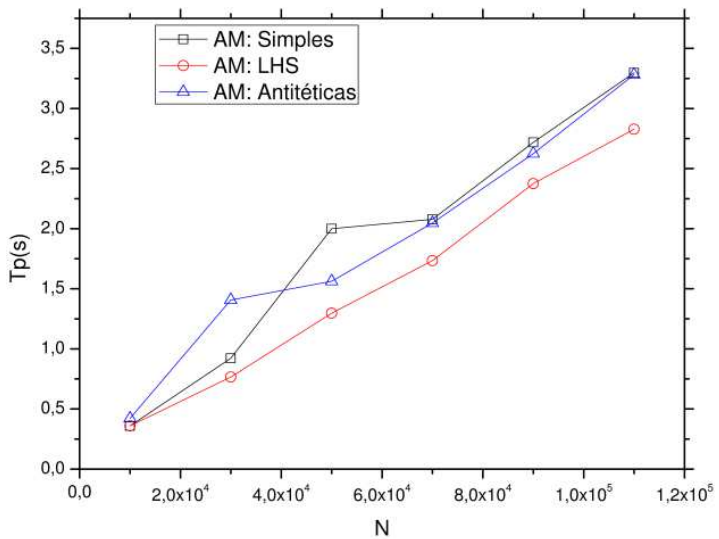

Figura 103. Relação entre o tempo de processamento e o tamanho da amostra no Exemplo 5: Amostragem Assintótica (a) e Amostragem Melhorada (b).

a) Simulação de Subconjunto

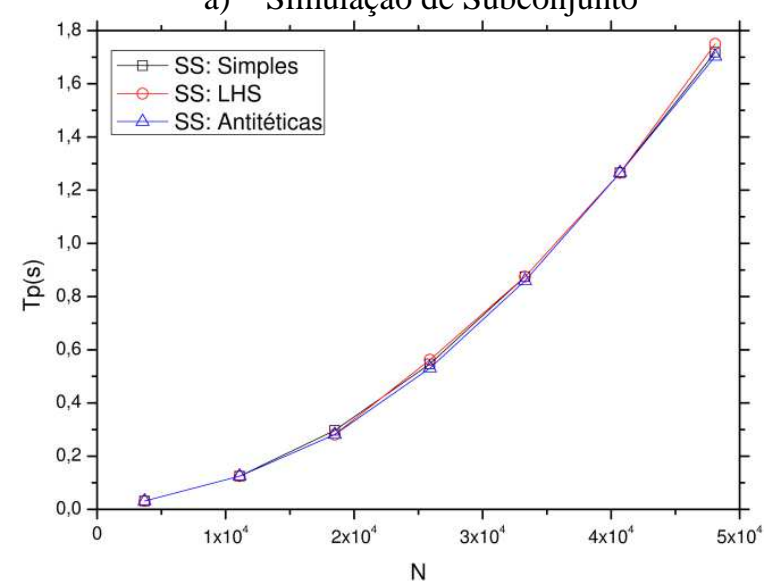

b) Todas as técnicas

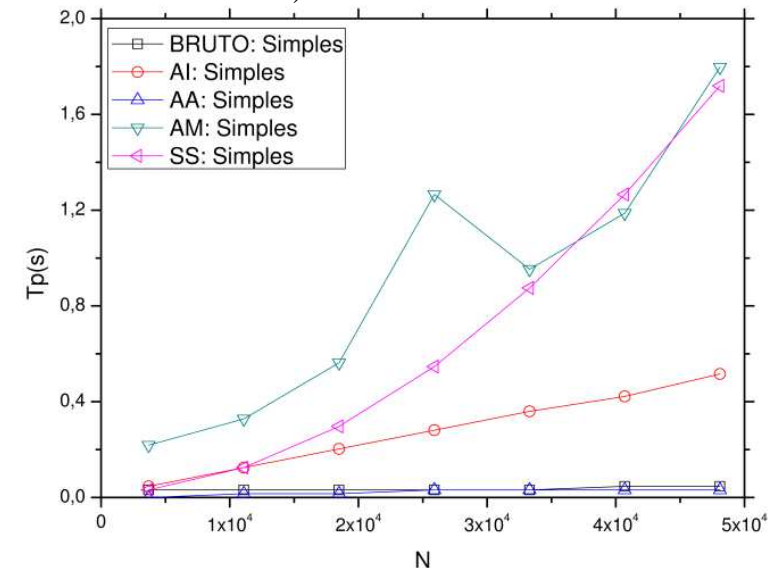

Figura 104. Relação entre o tempo de processamento e o tamanho da amostra no Exemplo 5: Simulação de Subconjuntos (a) e comparativo entre todas as técnicas(b).

De maneira geral, o comportamento observado neste exemplo é muito similar ao apresentado nos exemplos anteriores. Neste caso, a Amostragem por Hipercubo Latino e a Amostragem por Variáveis Antitéticas parece não ter uma forte influência no desempenho das técnicas. O que se nota de maneira clara é que as técnicas de Amostragem Melhorada, Amostragem por Importância e Simulação de Subconjuntos, apresentam altos tempos de processamento, se comparados com o Monte Carlo Bruto e com a Amostragem Assintótica. Porém, a Amostragem por Importância e a Simulação de Subconjuntos conseguem realizar uma boa estimativa da probabilidade de falha com uma amostra pequena.

\subsection{Exemplo 6: Torre em Elementos Finitos}

O objetivo deste problema é analisar as diferentes técnicas de simulação de Monte Carlo aqui estudadas e suas possíveis combinações, em conjunto com a análise de elementos finitos 
com não linearidade física e geométrica. Hansen e Vanderplaats (1990) analisaram uma torre de linha de transmissão de energia elétrica com carregamentos provenientes apenas dos cabos. Gomes e Beck (2013) apresentam uma modificação deste problema através da otimização de sua topologia (Figura 105) e da inserção das forças devido à ação do vento na estrutura. Assim, neste problema se considera uma estrutura ótima em relação à sua forma e aos custos associados (Gomes e Beck, 2013).

É realizado o acoplamento entre o módulo de simulação de Monte Carlo do StRAnD e o programa Acadframe, que é desenvolvido no departamento de engenharia de estruturas da EESC. Na modelagem da estrutura são considerados elementos de pórtico bidimensionais, com formulação posicional (CODA; PACCOLA, 2007. CODA; PACCOLA, 2010. CODA; PACCOLA, 2011. CODA; GRECO, 2004. GRECO e CODA, 2006. GRECO et al., 2006) para se realizar uma análise não linear geométrica. Cada elemento possui três nós, sendo dois extremos e um no meio do elemento. Cada nó possui três graus de liberdade, duas translações e uma rotação. São consideradas seções L, sendo o material considerado elástico perfeitamente plástico. A Figura 105 apresenta a torre que é o objeto de estudo neste exemplo, nela estão destacados os nós 11 e 12, pois estes nós são utilizados na estimativa do deslocamento médio que é utilizado na equação de estado limite, tal como será explicado a seguir.

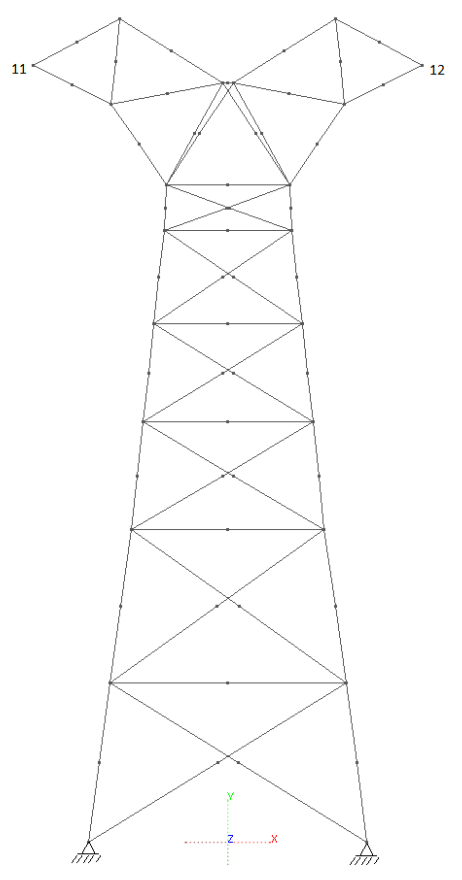

Figura 105. Torre analisada e nós de referência, para o Exemplo 6. 
A falha é caracterizada pela inclinação da curva que relaciona o fator de carga e o deslocamento (Figura 106). Onde o fator de carga é caracterizado por uma variação da carga em torno do seu valor atual.

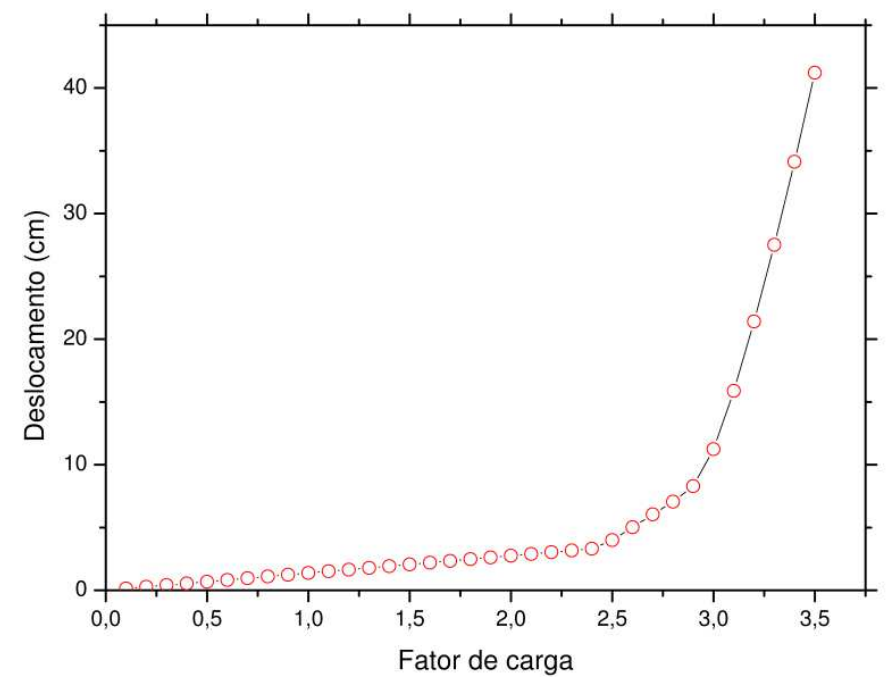

Figura 106. Diagrama fator de carga $\times$ deslocamento.

Assim, a equação de estado limite é dada por:

$$
g(\mathbf{x})=75^{\circ}-\operatorname{atan}\left(\frac{\Delta \delta}{\Delta L}\right)
$$

onde $\delta$ é o incremento no deslocamento médio entre os nós 11 e $12 ; L$ é o incremento no fator de carga adimensional, que é calculado a partir da carga atuante e $\mathbf{x}$ é o vetor que contêm as variáveis aleatórias. O ângulo crítico é de $75^{\circ}$, que é a inclinação do diagrama fator de carga $\times$ deslocamento, que representa a falha. A falha da estrutura não é representada necessariamente pelo seu colapso, mas sim por uma mudança do seu comportamento.

As cargas gravitacionais são definidas pelo peso próprio dos cabos. É considerada uma configuração simétrica dos cabos e consequentemente de suas cargas. A estimativa da ação horizontal de vento é baseada na NBR-6123: Forças devidas ao vento em edificações. O vento de projeto adotado é de $45 \mathrm{~m} / \mathrm{s}\left(\mathrm{v}_{0}=45 \mathrm{~m} / \mathrm{s}\right)$. A velocidade característica do vento, considerando os fatores topográficos $\left(S_{1}=1\right)$, de rugosidade $\left(S_{2}=1,06\right)$ e estatístico $\left(S_{3}=1\right)$, é dada por:

$$
v_{k}=S_{1} \cdot S_{2} \cdot S_{3} \cdot v_{0}=1 \times 1,06 \times 1 \times 45=47,7 \mathrm{~m} / \mathrm{s},
$$

onde o perfil de velocidade do vento com a altura segue a seguinte lei: 


$$
v(z)=\sqrt{0,1 \cdot z \cdot v_{k}^{2}}
$$

Para aplicar variabilidade ao vento é introduzida uma variável aleatória adimensional $V$ que segue a distribuição de Gumbel para máximos, tal que:

$$
Z(z)=v(z) \cdot V
$$

Os valores das médias e dos coeficientes de variação, das variáveis $V$ e $E$, são apresentados na Tabela 35. Para este problema, o coeficiente de arrasto é $C_{a}=2,1$, que é o valor máximo para perfis $\mathrm{L}$.

Tabela 35. Médias e coeficientes de variação das variáveis aleatórias do Exemplo 6.

\begin{tabular}{clll}
\hline Variável Aleatória & Distribuição & Média & C.V \\
\hline $\boldsymbol{V}$ & Gumbel para máximos & 0,95 & 0,13 \\
$\boldsymbol{E}$ & Lognormal & $207 \mathrm{GPa}$ & 0,03 \\
\hline
\end{tabular}

A pressão do vento é avaliada pela seguinte equação:

$$
q(z, V)=0,613 \cdot(v(z) \cdot V)^{2} .
$$

Neste trabalho são considerados cabos de $2,52 \mathrm{~cm}$ de diâmetro, cuja área de influência é admitida como $A=300 \mathrm{~m} \times 0,0252 \mathrm{~m}=7,56 \mathrm{~m}^{2}$. O coeficiente de arrasto devido aos cabos é de $C_{a}=1,2$. Assim, a velocidade média do vento que age no cabo, obtida por meio da Eq. (147), é de aproximadamente $v\left(z_{\text {cabos }}\right)=58,88 \mathrm{~m} / \mathrm{s}$. Considera-se que os cabos se encontram na cota $z_{\text {cabos }}=15,24 \mathrm{~m}$, a partir do solo. Como a força de arrasto é calculada por $F_{a}=C_{a} \cdot A \cdot q(z, V)=1,2 \cdot 7,56 \cdot 0,613 \cdot(58,88 \cdot V)^{2}=19279,65 \cdot V^{2}$. Assim, a força de arrasto média que atua nos cabos é de $17,4 \mathrm{kN}$.

Neste exemplo é analisado o desempenho das técnicas aqui estudadas em relação ao estado limite definido pela Eq. (145). Inicialmente é realizada a análise do problema através do FORM. Dessa forma, é possível obter uma estimativa da grandeza do valor da probabilidade de falha, uma vez que neste problema o tamanho da amostra utilizada na simulação de Monte Carlo é de grande importância. O tempo de processamento do FORM foi de 29,8 segundos, onde houve 31 chamadas da equação de estado limite. O valor inicial do ponto de projeto foi $x^{*}=(0,950 ; 20.684,27726)$, sendo $x^{*}=(1,8 ; 20.505,4)$ o ponto de projeto encontrado. Assim, foi obtida a probabilidade de falha de $P_{f, F O R M}=1,088 \times 10^{-4}$ e o índice de confiabilidade de $\beta=3,6976$. A utilização do FORM pode levar a uma boa estimativa da probabilidade de falha, pois neste problema o limite de falha é aproximadamente linear, tal 
como será visto adiante. Porém, é interessante testar este valor com a simulação de Monte Carlo.

A maior dificuldade apresentadas pelas técnicas de simulação de Monte Carlo é a necessidade de se realizar avaliações de um problema numérico iterativo, como neste caso, em que se utiliza o Método dos Elementos Finitos na presença de não linearidades. Inicialmente são abordados os desempenhos do Monte Carlo Bruto e da Amostragem por Importância. Em seguida são realizados estudos referentes à Amostragem Assintótica, Amostragem Melhorada e da Simulação de Subconjuntos. Por fim, é realizada uma análise comparativa da convergência e do tempo de processamento das técnicas aqui estudadas.

São realizadas simulações do Monte Carlo Bruto e do Monte Carlo com Amostragem por importância, de forma que o resultado do FORM possa ser testado. A Tabela 36 e a Tabela 37 apresentam os resultados da probabilidade de falha, do coeficiente de variação da probabilidade de falha, do tempo de processamento e do erro relativo à resposta do FORM, para o método de Monte Carlo Bruto e para o método de Monte Carlo com Amostragem por Importância. Estes resultados são obtidos com uma amostra de tamanho $1 \times 10^{5}$ e utilizando como amostragem básica, a Amostragem Simples, a Amostragem por Hipercubo Latino e a Amostragem por Variáveis Antitéticas.

Tabela 36. Comparativo do uso de diferentes técnicas de amostragem básica no método de Monte Carlo Bruto,

\begin{tabular}{lcccc}
\multicolumn{5}{c}{ para uma amostra de $1 \times 10^{5}$, no exemplo 6.} \\
\hline \multicolumn{5}{c}{$\boldsymbol{P}_{\boldsymbol{f}}$ de referência: $\mathbf{1 , 0 8 8 \times \mathbf { 1 0 } ^ { - 4 }}$} \\
Simples & $\boldsymbol{P}_{\boldsymbol{f}}$ & C.V. da $\boldsymbol{P}_{\boldsymbol{f}}$ & Tp & Erro Relativo (\%) \\
LHS & $1,400 \times 10^{-4}$ & 0,2672 & $11 \mathrm{~h}$ e $2 \mathrm{~min}$ & 28,68 \\
Antitética & $1,10 \times 10^{-4}$ & 0,3015 & $10 \mathrm{~h} \mathrm{e} 17 \mathrm{~min}$ & 1,10 \\
\hline
\end{tabular}

Tabela 37. Comparativo do uso de diferentes técnicas de amostragem básica no método de Monte Carlo com Amostragem por Importância, para uma amostra de $1 \times 10^{5}$, no exemplo 6 .

\begin{tabular}{lcccc}
\hline \multicolumn{5}{c}{$\boldsymbol{P}_{\boldsymbol{f}}$ de referência: $\mathbf{1 , 0 8 8 \times \mathbf { 1 0 } ^ { - 4 }}$} \\
\hline Simples & $\boldsymbol{P}_{\boldsymbol{f}}$ & $\mathbf{C . V . d a} \boldsymbol{P}_{\boldsymbol{f}}$ & $\mathbf{T p}$ & Erro Relativo (\%) \\
LHS & $1,099 \times 10^{-4}$ & 0,0038 & $13 \mathrm{~h}$ e $36 \mathrm{~min}$ & 1,01 \\
Antitética & $1,089 \times 10^{-4}$ & 0,0038 & $13 \mathrm{~h} \mathrm{e} 41 \mathrm{~min}$ & 0,09 \\
\hline
\end{tabular}

onde Tp é o tempo de processamento da análise em questão para cada uma das técnicas utilizadas.

Como se observa na Tabela 36, a simulação pelo método de Monte Carlo Bruto apresentou resultados próximos do valor obtido pelo FORM, com destaque para o uso 
conjunto com a Amostragem por Hipercubo Latino, porém, o uso de Amostragem por Importância (Tabela 37) apresentou resultados melhores que o apresentado pelo método de Monte Carlo Bruto. Além disso, a Amostragem por Importância apresenta um menor coeficiente de variação da probabilidade de falha, apesar de apresentar um tempo de processamento maior. Portanto, o resultado obtido pelo FORM será utilizado como referência nesse trabalho.

A seguir serão apresentados os estudos referentes as técnicas de Amostragem Assintótica, Amostragem Melhorada, Simulação de Subconjuntos e por fim será apresentado um estudo comparativo entre as técnicas.

\subsubsection{Amostragem Assintótica}

É realizado um comparativo dos gráficos de regressão obtidos pela técnica de Amostragem Assintótica (Figura 107). É utilizada uma amostra de tamanho $1 \times 10^{5}$, com a Amostragem Simples, a Amostragem por Hipercubo Latino e a Amostragem por Variáveis Antitéticas. O parâmetro $f$ foi variado de 0,6 a 0,9 , onde são utilizados 5 pontos de suporte.

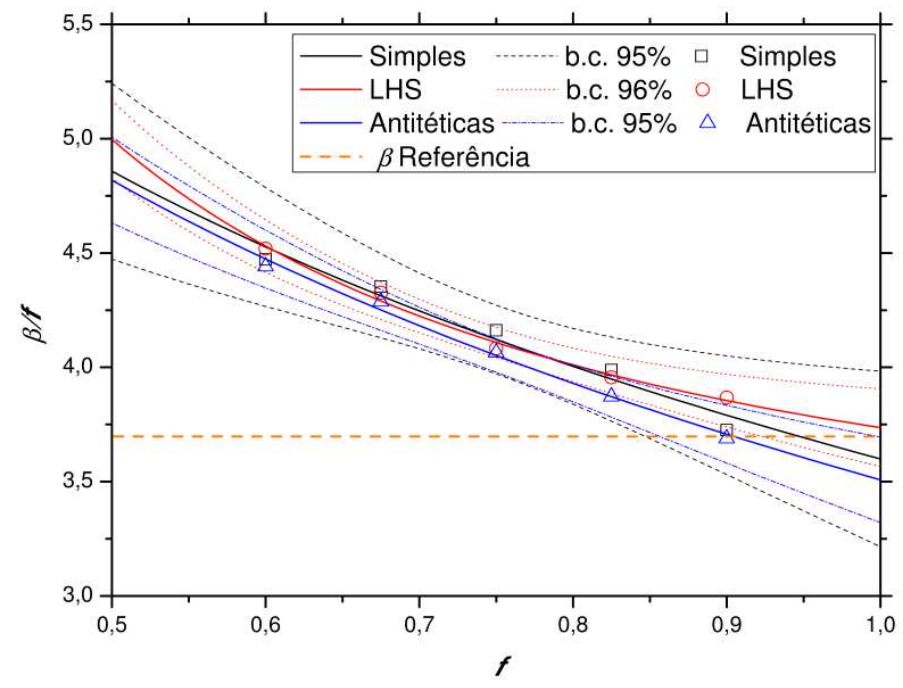

Figura 107. Regressão não linear utilizada na Amostragem Assintótica, para amostras de tamanho $1 \times 10^{5}$, no Exemplo 6.

Os parâmetros ajustados na técnica em questão, com a utilização da Amostragem Simples (ASMC), da Amostragem por Hipercubo Latino (ALHS) e da Amostragem por Variáveis Antitéticas (AASMC), são apresentados na Tabela 38. 
Tabela 38. Parâmetros ajustados na Amostragem Assintótica para o Exemplo 6.

\begin{tabular}{cccc}
\hline $\boldsymbol{N}$ & \multicolumn{3}{c}{$\mathbf{1 \times \mathbf { 1 0 } ^ { \mathbf { 5 } }}$} \\
\hline & $A$ & $B$ & $C$ \\
ASMC & $-15.917,960$ & $15.921,560$ & $-1,1401 \times 10^{-4}$ \\
ALHS & 3,0455 & 0,6909 & $-1,498$ \\
AASMC & $-9.240,556$ & $9.244,064$ & $-2,0447 \times 10^{-4}$ \\
\hline
\end{tabular}

De acordo com a Figura 107, se observa uma boa concordância entre as curvas ajustadas e os pontos de suporte, com destaque para o uso conjunto com a Amostragem por Hipercubo Latino, onde se observam bandas de confiança mais estreitas no ajuste realizado. Além disso, tal resultado pode ser comprovado na Tabela 39, onde se observa que o uso de Amostragem por Hipercubo Latino leva a um menor erro na estimativa da probabilidade de falha.

Tabela 39. Índice de confiabilidade, probabilidade de falha, coeficiente de variação da probabilidade de falha e tempo de processamento, para a Amostragem Assintótica, no Exemplo 6.

\begin{tabular}{lcccc}
\hline \multicolumn{5}{c}{$\boldsymbol{P}_{\boldsymbol{f}}$ de referência: $\mathbf{1 , 0 8 8 \times \mathbf { 1 0 } ^ { - 4 }}$} \\
\hline Simples & $\boldsymbol{P}_{\boldsymbol{f}}$ & $\mathbf{C . V . d a} \boldsymbol{P}_{\boldsymbol{f}}$ & $\mathbf{T p}$ & Erro Relativo (\%) \\
LHS & $1,60 \times 10^{-4}$ & 1,5734 & $12 \mathrm{~h} \mathrm{e} 22 \mathrm{~min}$ & 47,0588 \\
Antitética & $9,33 \times 10^{-5}$ & 0,4791 & $11 \mathrm{~h} \mathrm{e} 19 \mathrm{~min}$ & 14,2463 \\
\hline
\end{tabular}

\subsubsection{Amostragem Melhorada}

Na Figura 108 observa-se uma boa concordância entre a curva ajustada e os pontos de suporte, para uma amostra de tamanho $1 \times 10^{5}$. Nesse caso foram utilizados 100 pontos de suporte. O parâmetro $\lambda$ varia no intervalo de 0,4 a 0,9 .

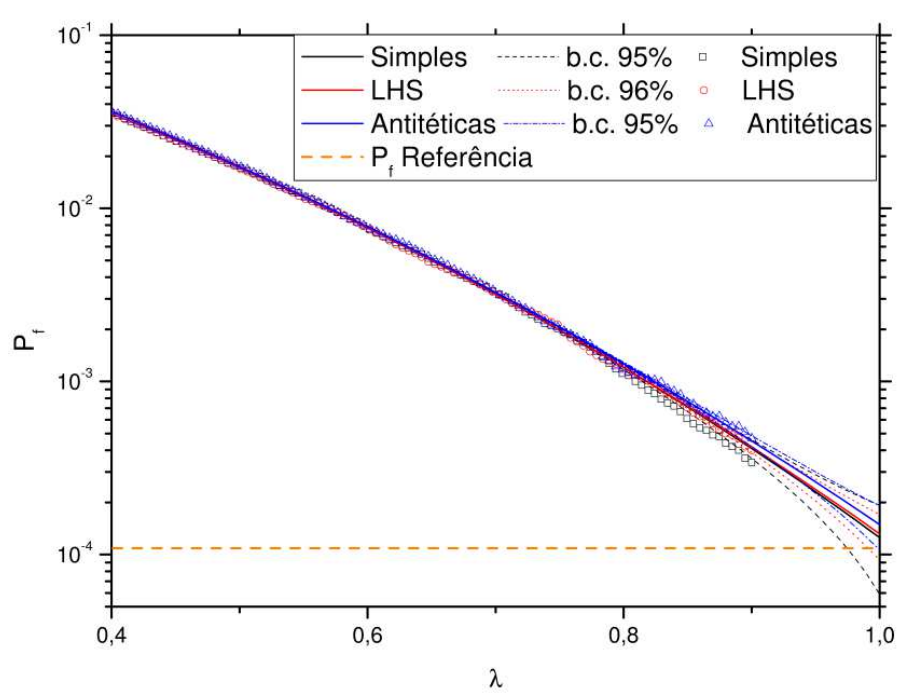

Figura 108. Regressão não linear utilizada na Amostragem Melhorada para amostras de tamanho $1 \times 10^{5}$, no Exemplo 6. 
Os parâmetros ajustados na técnica em questão, com a utilização da Amostragem Simples (AM), da Amostragem por Hipercubo Latino (AM_LHS) e da Amostragem por Variáveis Antitéticas (AAM), são apresentados na Tabela 40.

Tabela 40. Parâmetros ajustados na Amostragem Melhorada, para uma amostra de tamanho $1 \times 10^{5}$, no Exemplo

\begin{tabular}{ccccc}
\hline & $\boldsymbol{q}$ & $\boldsymbol{a}$ & $\boldsymbol{b}$ & $\boldsymbol{c}$ \\
\hline AM & 6,2375 & $2,2002 \times 10^{-3}$ & $-3,7501$ & 5,4552 \\
AM_LHS & 4,8546 & $9,2206 \times 10^{-2}$ & $-2,3842$ & 3,8852 \\
A_AM & 9,5191 & $8,5025 \times 10^{-2}$ & $-2,6185$ & 3,7857 \\
\hline
\end{tabular}

A Tabela 41, apresenta os valores das probabilidades de falha, dos coeficientes de variação das probabilidades de falha, do tempo de processamento e do erro relativo à resposta do FORM, para diferentes técnicas de amostragem básica.

Tabela 41. Índice de confiabilidade, probabilidade de falha, coeficiente de variação da probabilidade de falha e tempo de processamento para a Amostragem Melhorada, no Exemplo 6.

\begin{tabular}{|c|c|c|c|c|}
\hline \multicolumn{5}{|c|}{$P_{f}$ de referência: $1,088 \times 10^{-4}$} \\
\hline & $\boldsymbol{P}_{f}$ & C.V. da $P_{f}$ & $\mathbf{T p}$ & Erro Relativo (\%) \\
\hline Simples & $1,254 \times 10^{-4}$ & 0,2710 & $10 \mathrm{~h}$ e $2 \mathrm{~min}$ & 15,2574 \\
\hline LHS & $1,317 \times 10^{-4}$ & 0,1502 & $9 \mathrm{~h} \mathrm{e} 46 \mathrm{~min}$ & 21,0478 \\
\hline Antitética & $1,489 \times 10^{-4}$ & 0,1460 & $9 \mathrm{~h} \mathrm{e} 46 \mathrm{~min}$ & 36,8566 \\
\hline
\end{tabular}

Na Tabela 41, observa-se que a Amostragem Melhorada apresenta uma boa redução do coeficiente de variação da probabilidade de falha e resultados estimados mais consistentes da probabilidade de falha. Vale ressaltar que a utilização de Amostragem por Hipercubo Latino e Amostragem por Variáveis Antitéticas se mostrou vantajosa neste caso.

\subsubsection{Simulação de Subconjuntos}

Os dados de entrada do StRAnD, que definem o caminhante aleatório utilizado na Simulação de Subconjuntos, são apresentados na Tabela 42.

Tabela 42. Médias e desvios padrão do caminhante aleatório do Exemplo 6.

\begin{tabular}{clll}
\hline Variável Aleatória & Distribuição & Média & Desvio-Padrão \\
\hline $\boldsymbol{V}$ & Uniforme & 0,0 & 0,06175 \\
$\boldsymbol{E}$ & Uniforme & 0,0 & $3,105 \mathrm{MPa}$ \\
\hline
\end{tabular}

Os valores de desvio padrão apresentados na Tabela 42, correspondem à metade dos desvios padrão do problema original. Logo, com 4.000 pontos amostrais em cada subconjunto e com $P_{0}=0,1$, é possível se obter a Figura 109, a Figura 110 e a Figura 111, onde é ilustrada a formação de quatro subconjuntos, com o primeiro subconjunto gerado pela Amostragem 
Simples (Figura 109), pela Amostragem por Hipercubo Latino (Figura 110) e pela Amostragem por Variáveis Antitéticas (Figura 111).

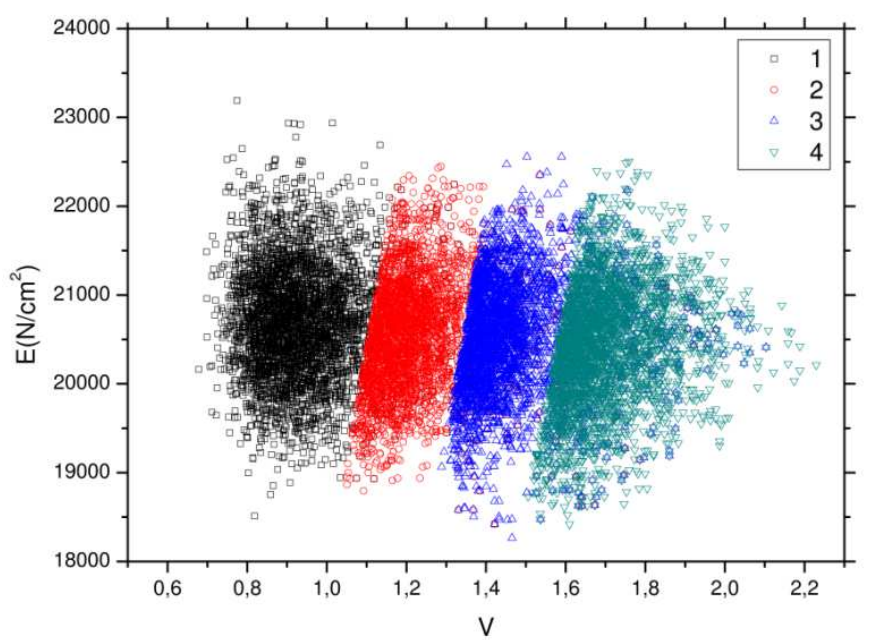

Figura 109. Simulação de Subconjuntos: Amostragem Simples.

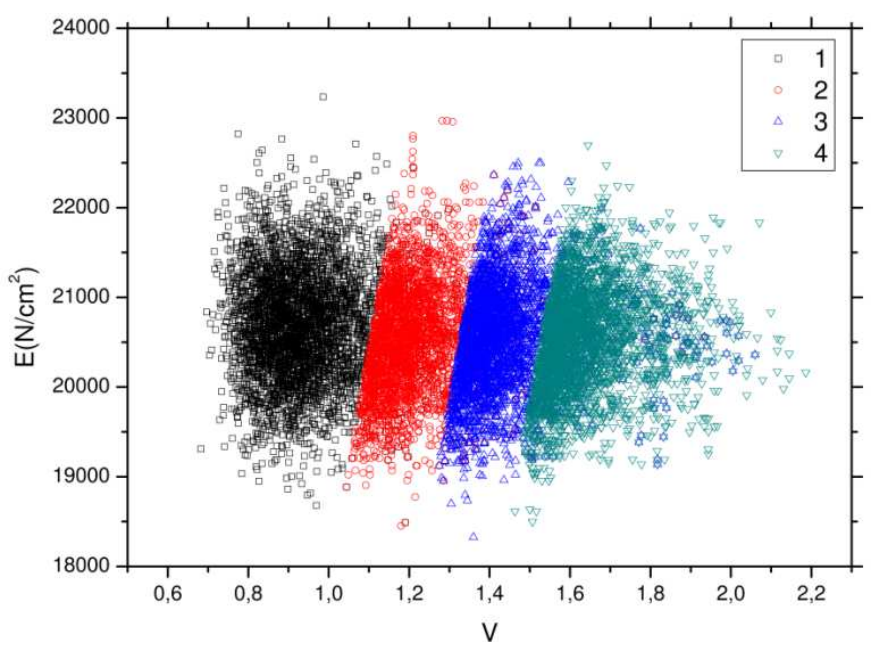

Figura 110. Simulação de Subconjuntos: Amostragem por Hipercubo Latino. 


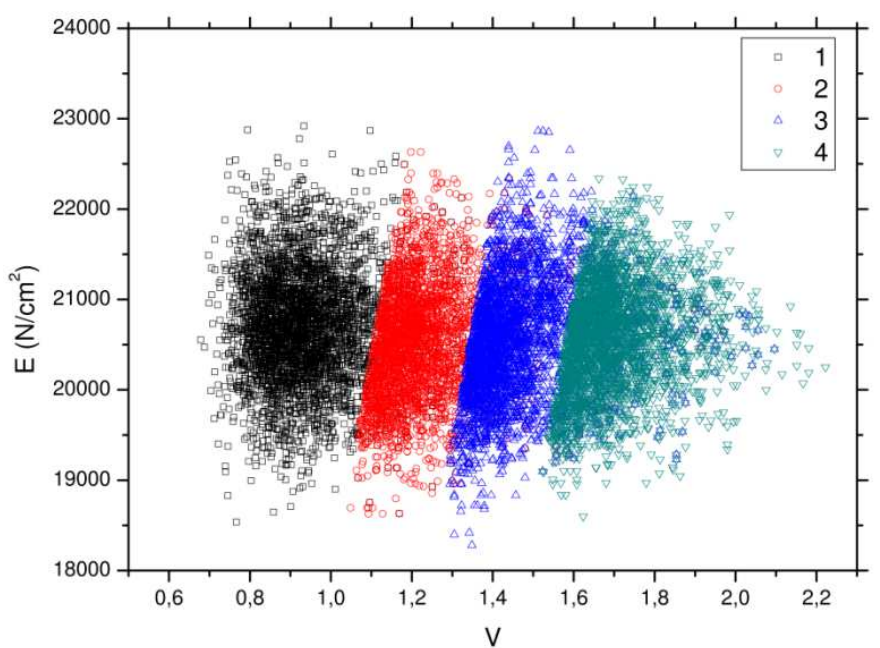

Figura 111. Simulação de Subconjuntos: Amostragem por Variáveis Antitéticas.

A partir da Figura 109, da Figura 110 e da Figura 111, primeiramente se observa que a Simulação de Subconjuntos pode ser aplicada sem maiores dificuldades. Segundo, é possível notar que a equação de estado limite é quase linear, ou pouco não linear. Portanto, o FORM deverá apresentar uma boa estimativa para a probabilidade de falha, por isso, o valor da probabilidade de falha obtida pelo FORM é utilizado como probabilidade de falha de referência.

\subsubsection{Análise de convergência}

É realizado um comparativo entre o Monte Carlo Bruto, a Amostragem por Importância, a Amostragem Assintótica e a Amostragem Melhorada. As técnicas estudadas fazem uso da Amostragem Simples, da Amostragem por Hipercubo Latino e da Amostragem por Variáveis Antitéticas. Variou-se o tamanho da amostra de $1 \times 10^{3}$ até $1 \times 10^{5}$, tomando-se nesse intervalo 100 pontos.

a) Média

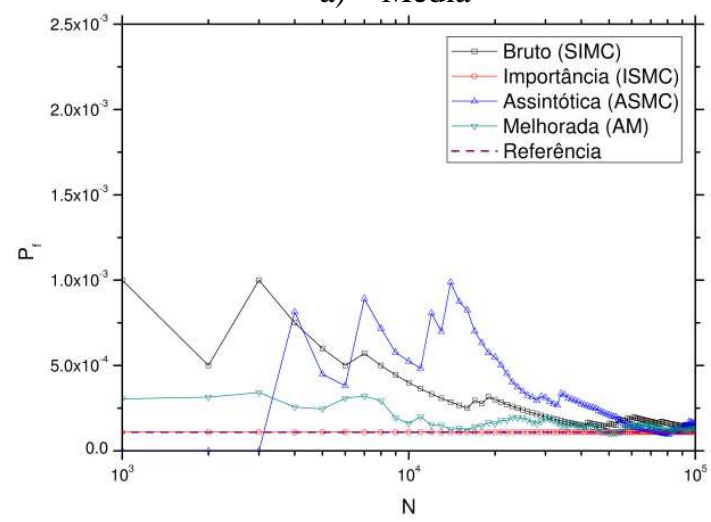

b) C.V.

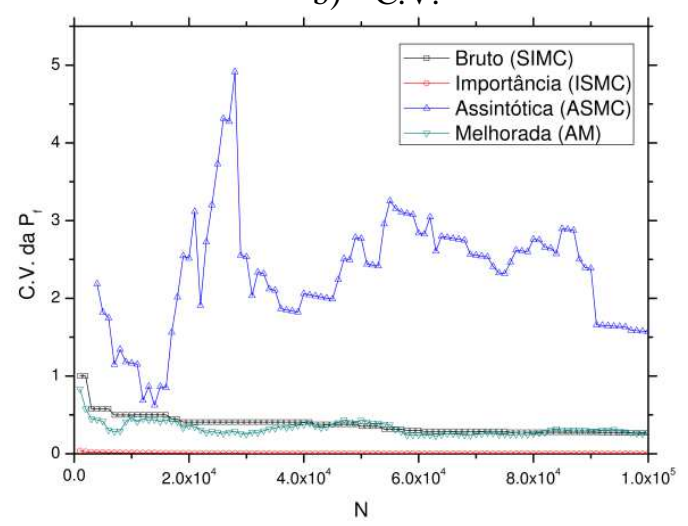

Figura 112. Comparativo da convergência da $P_{f}$ (a) e de C.V. (b) para a Amostragem Simples, no Exemplo 6. 
a) Média

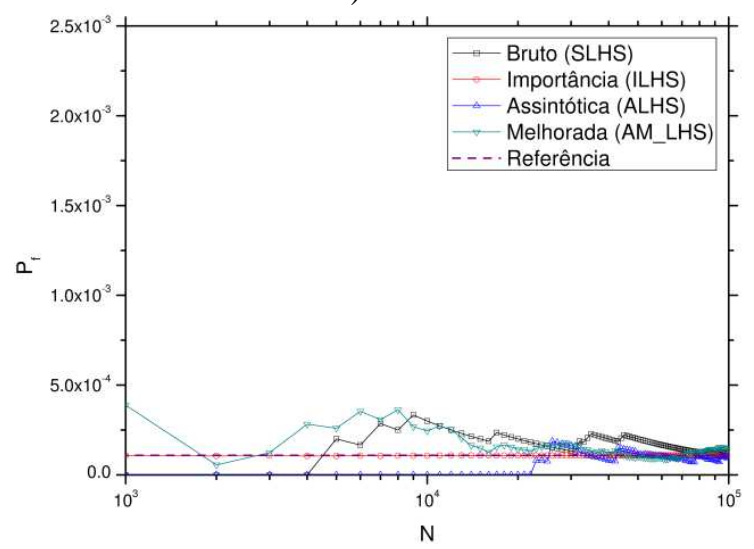

b) C.V.

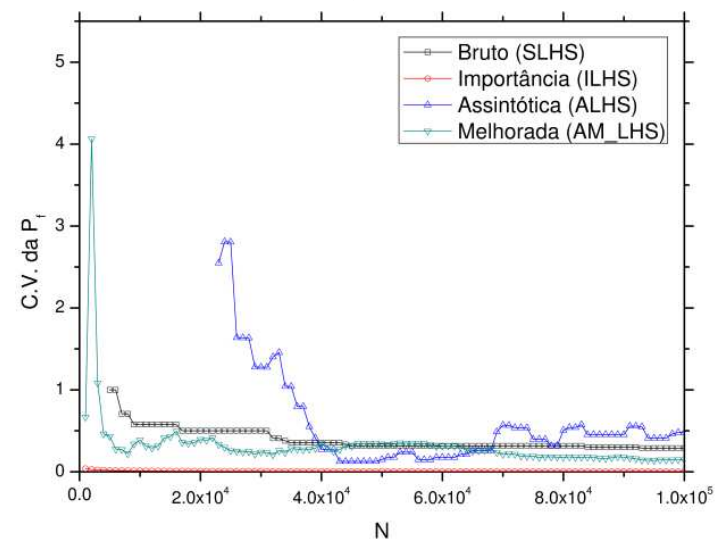

Figura 113. Comparativo da convergência da $P_{f}$ (a) e de C.V. (b) para a Amostragem por Hipercubo Latino, no Exemplo 6.

a) Média

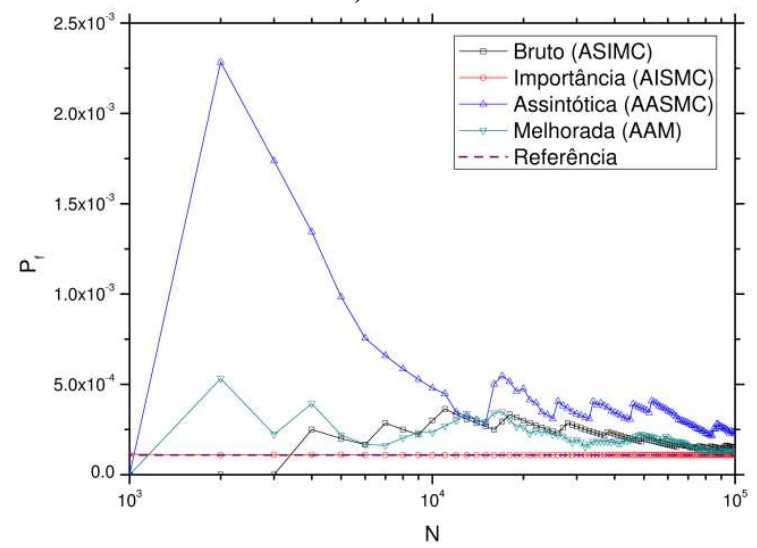

b) C.V.

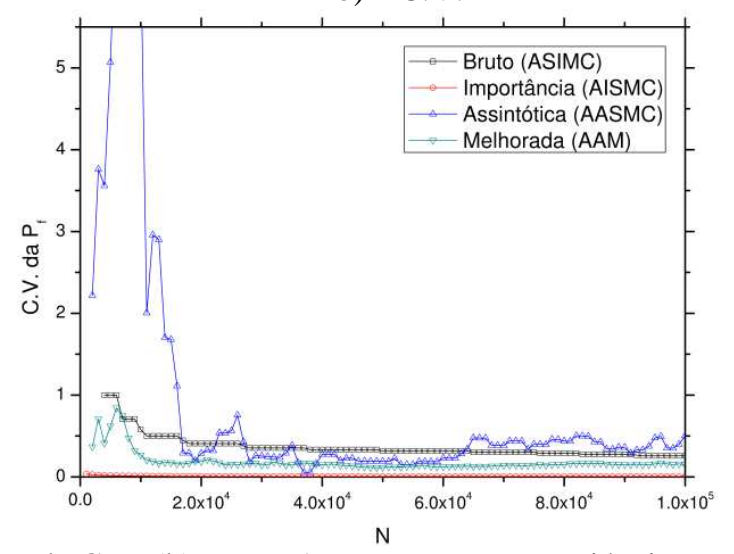

Figura 114. Comparativo da convergência da $P_{f}($ a) e de C.V. (b) para a Amostragem por Variáveis Antitéticas, no Exemplo 6.

Pode-se notar que as técnicas em questão tendem a convergir para o mesmo ponto, que é representado pela linha de valor de referência da probabilidade de falha. Valor este obtido por meio do FORM. De acordo com a Figura 112a e Figura 112b, pode-se notar que a Amostragem por Importância apresenta uma convergência mais rápida do que as outras técnicas, como já esperado. Por outro lado, observa-se que a técnica de Amostragem Melhorada apresenta uma convergência mais rápida que o Monte Carlo Bruto e a Amostragem Assintótica, além de apresentar um coeficiente de variação da probabilidade de falha muito próximo do obtido pela simulação do Monte Carlo Bruto. Vale destacar que a técnica de Amostragem Assintótica com Amostragem Simples, apesar de convergir para o resultado esperado, não o fez de maneira satisfatória, além de apresentar uma forte flutuação do coeficiente de variação da probabilidade de falha. 
Aplicando as técnicas de Amostragem por Hipercubo Latino (Figura 113) e Amostragem por Variáveis Antitéticas (Figura 114), se observa que a técnica de Amostragem Melhorada apresentou um bom desempenho em termos de convergência da probabilidade de falha e de redução do seu coeficiente de variação. Sua resposta só não foi melhor que a da Amostragem por Importância.

A seguir são apresentados os gráficos do comparativo entre as técnicas estudadas, com aplicação de diferentes técnicas de amostragem básica. Neste caso é feito um gráfico para cada uma das técnicas (Bruto, Amostragem por Importância, Amostragem Assintótica e Amostragem Melhorada) e para cada técnica são obtidas três curvas, sendo uma para cada amostragem básica (Amostragem Simples, Amostragem por Hipercubo Latino e Amostragem por Variáveis Antitéticas).

a) Média

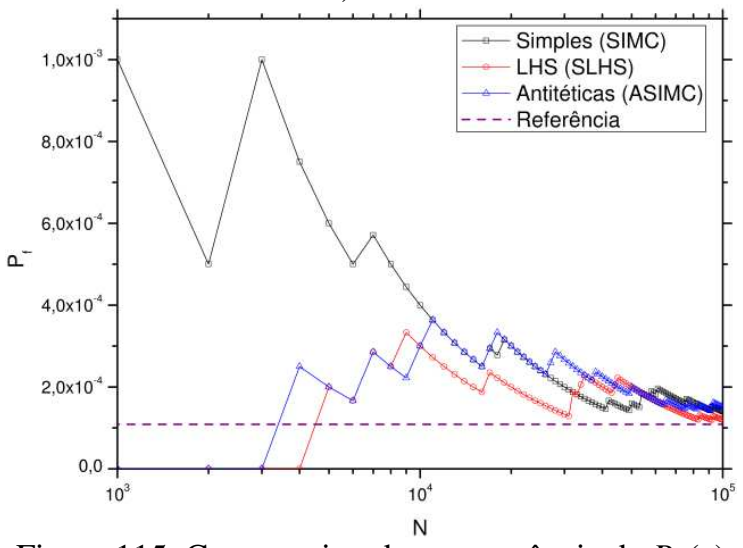

b) C.V.

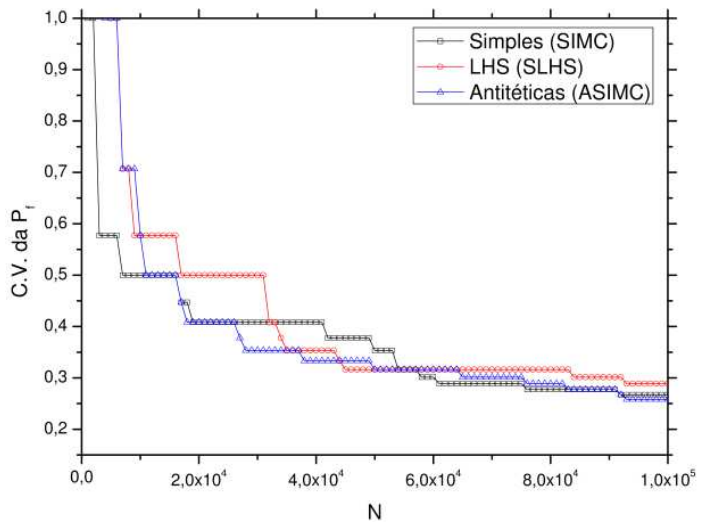

Figura 115. Comparativo da convergência da $P_{f}(a)$ e de C.V. (b) para o Monte Carlo Bruto, no Exemplo 6.

a) Média

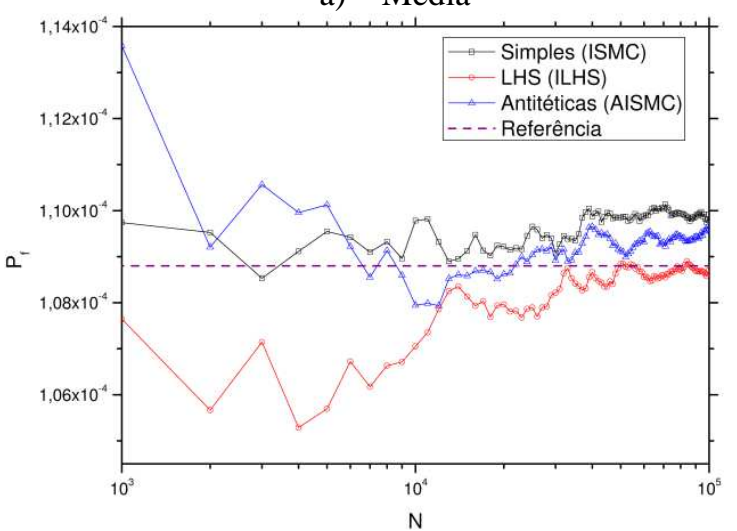

b) C.V.

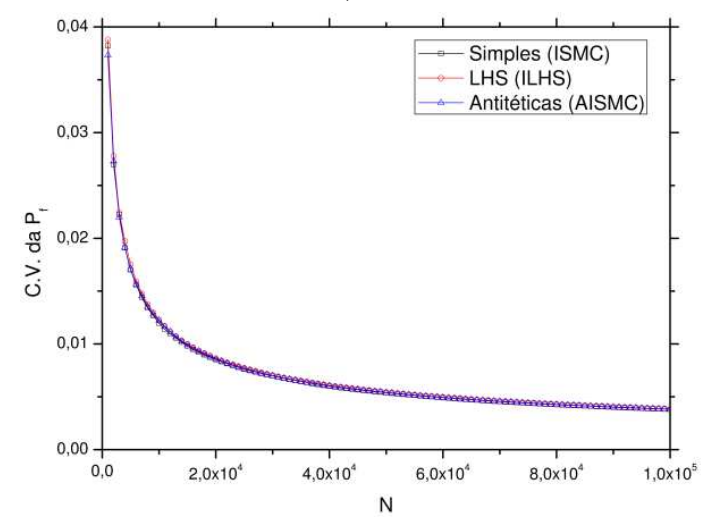

Figura 116. Comparativo da convergência da $P_{f}($ a) e de C.V. (b) para a Amostragem por Importância, no Exemplo 6. 
a) Média

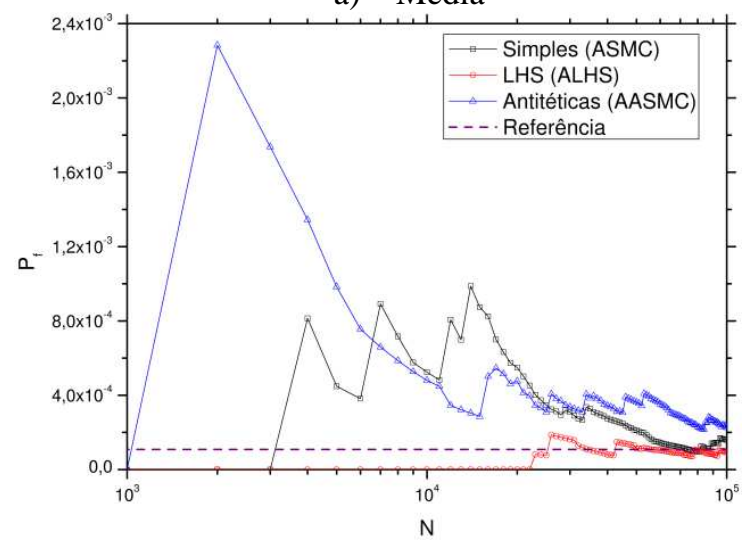

b) C.V.

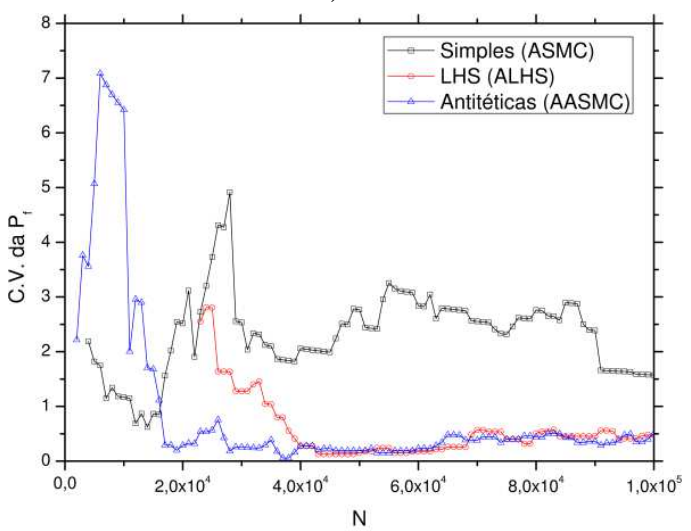

Figura 117. Comparativo da convergência da $P_{f}($ a) e de C.V. (b) para a Amostragem Assintótica, no Exemplo 6.

a) Média

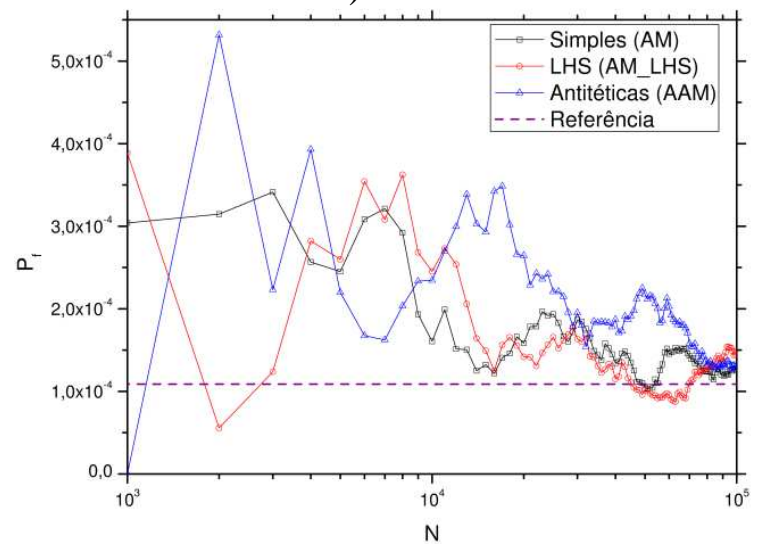

b) C.V.

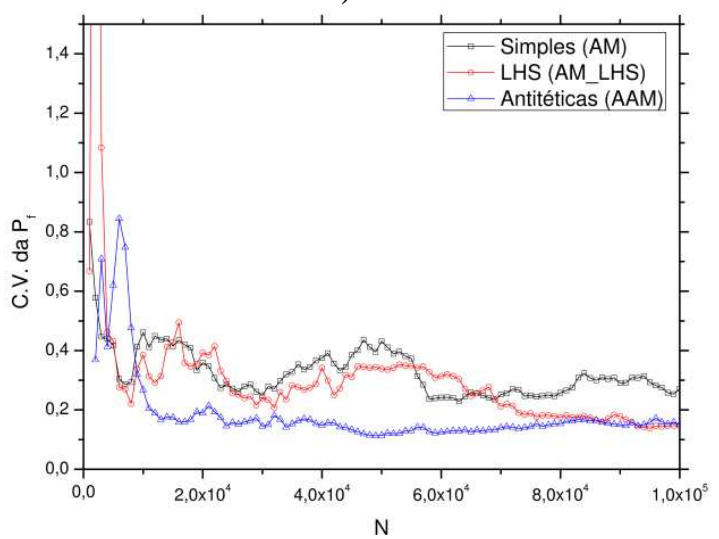

Figura 118. Comparativo da convergência da $P_{f}$ (a) e de C.V. (b) para a Amostragem Melhorada, no Exemplo 6.

Na Figura 115a, observa-se que o uso da Amostragem por Hipercubo Latino e da Amostragem por Variáveis Antitéticas, no método de Monte Carlo Bruto, leva á uma convergência mais rápida da probabilidade de falha para amostras pequenas, porém, o uso da Amostragem Simples permitiu que falhas fossem observadas para amostras menores que $2 \times 10^{3}$. Por outro lado, na Figura 115 b não se observa vantagem significativa, em relação ao coeficiente de variação da probabilidade de falha, devido ao uso da Amostragem por Hipercubo Latino e da Amostragem por Variáveis Antitéticas.

Na Figura 116a, observa-se que o uso da Amostragem por Hipercubo Latino só foi vantajoso para a Amostragem por Importância, para amostras maiores que $3 \times 10^{4}$. Na Figura 116b, percebe-se que os coeficientes de variação da probabilidade de falha são próximos.

Na Figura 117a, observa-se que a Amostragem Assintótica com Hipercubo Latino, apresenta um bom resultado para amostras maiores que $2 \times 10^{4}$, por outro lado, o uso da 
Amostragem por Variáveis Antitéticas não é tão vantajoso em termos de convergência da probabilidade de falha, porém, tanto a Amostragem por Hipercubo Latino, quanto a Amostragem por Variáveis Antitéticas, apresentam redução do coeficiente de variação da probabilidade de falha (Figura 117b), em comparação com a Amostragem Simples.

Na Figura 118a, não se observa vantagem aparente no uso da Amostragem por Hipercubo Latino e da Amostragem por Variáveis Antitéticas, em conjunto com a Amostragem Melhorada. A não ser pela leve redução do coeficiente de variação da probabilidade de falha (Figura 118b) com o uso da Amostragem por Variáveis Antitéticas, o que não é significativo.

\subsubsection{Comparativo da probabilidade de falha e de seu coeficiente de variação}

Neste estudo é utilizada uma amostra de tamanho 14.800 para todos os problemas aqui estudados. Na Simulação de Subconjuntos, considera-se 4.000 pontos amostrais em cada subconjunto, além disso, os parâmetros do caminhante aleatório são os mesmos que foram utilizados em 7.6.3. Tais resultados podem ser observados na Tabela 43.

Tabela 43. Comparativo da $P_{f}$ para uma amostra de tamanho 14.800, no Exemplo 6.

\begin{tabular}{ccccc}
\hline \multicolumn{5}{c}{ SIMPLES } \\
\hline Sigla & Técnica & $\boldsymbol{P}_{\boldsymbol{f}}$ & $\boldsymbol{C} . \boldsymbol{V} . \boldsymbol{d a} \boldsymbol{P}_{\boldsymbol{f}}$ & Erro(\%) \\
BRUTO & Simples & $2,703 \times 10^{-4}$ & 0,4999 & 148,44 \\
AI & Importância & $1,090 \times 10^{-4}$ & 0,0099 & 0,18 \\
AA & Assintótica & $2,697 \times 10^{-4}$ & 8,0852 & 147,89 \\
AM & Melhorada & $1,327 \times 10^{-4}$ & 0,4103 & 21,97 \\
SS & Subconjuntos & $1,960 \times 10^{-4}$ & 0,1970 & 80,15 \\
& HIPERCUBO LATINO & & \\
Sigla & Técnica & $\boldsymbol{P}_{\boldsymbol{f}}$ & $\boldsymbol{C} . \boldsymbol{V} . \boldsymbol{d a} \boldsymbol{P}_{\boldsymbol{f}}$ & Erro (\%) \\
BRUTO & Simples & $\mathbf{6 , 7 5 7 \times 1 0 ^ { - 5 }}$ & -- & 37,90 \\
AI & Importância & $1,091 \times 10^{-4}$ & 0,0100 & 0,28 \\
AA & Assintótica & $8,300 \times 10^{-5}$ & 1,5114 & 23,71 \\
AM & Melhorada & $1,659 \times 10^{-4}$ & 0,3106 & 52,48 \\
SS & Subconjuntos & $1,110 \times 10^{-4}$ & 0,2046 & 2,02 \\
Sigla & VARIÁVEIS ANTITÉTICAS & & \\
BRUTO & Técnica & $\boldsymbol{P}_{\boldsymbol{f}}$ & $\boldsymbol{C} . \boldsymbol{V} . \boldsymbol{d a} \boldsymbol{P}_{\boldsymbol{f}}$ & Erro (\%) \\
AI & Simples & $2,703 \times 10^{-4}$ & 0,4999 & 148,44 \\
AA & Importância & $1,086 \times 10^{-4}$ & 0,0100 & 0,18 \\
AM & Assintótica & $1,393 \times 10^{-4}$ & 4,7499 & 28,03 \\
SS & Melhorada & $1,650 \times 10^{-4}$ & 0,3941 & 51,65 \\
& Subconjuntos & $1,623 \times 10^{-4}$ & 0,1963 & 49,17 \\
\hline
\end{tabular}


O resultado da Tabela 43 é ilustrado graficamente na Figura 119. De maneira geral, observa-se que a utilização da Amostragem por Hipercubo Latino diminui o erro da estimativa da probabilidade de falha em quase todas as técnicas. Também se observa que com uma amostra de tamanho 14.800, foi possível obter um coeficiente de variação menor que aquele apresentado pelo Monte Carlo Bruto com uma amostra de tamanho $1 \times 10^{5}$.

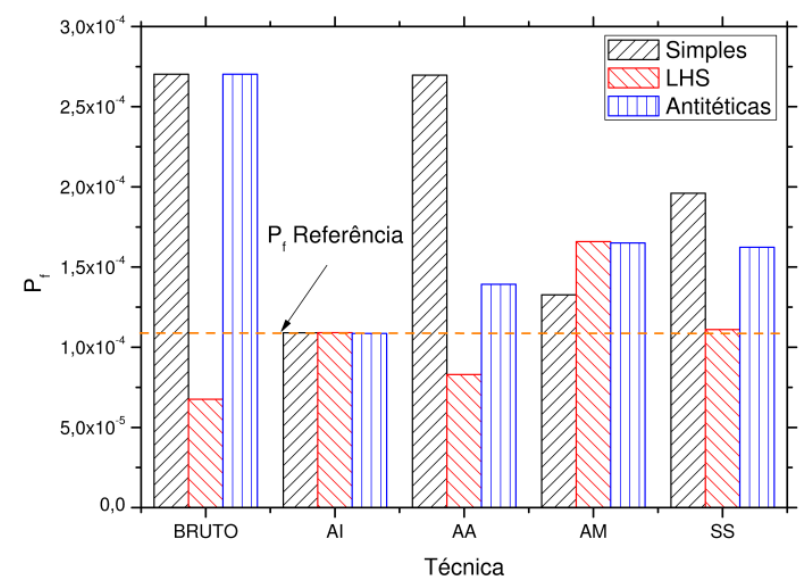

Figura 119. Comparativo da probabilidade de falha para uma amostra de tamanho 14.800, no Exemplo 6.

Um teste adicional é realizado com a Simulação de Subconjuntos, de forma a testar a potencialidade da técnica para uma amostra menor do que a estudada anteriormente. Neste caso, considera-se um caminhante aleatório cujo desvios padrão são o dobro dos desvios padrãodo problema original $(\alpha=2)$. Além disso, considera-se $N_{S S}=2.000$ e $P_{0}=0,1$, tais parâmetro são bons candidatos quando não se tem muito conhecimento do problema, como neste caso, onde realizar alguns testes pode ser computacionalmente custoso. Assim, foi possível obter os seguintes resultados:

Tabela 44. Comparativo da $P_{f}$, no Exemplo 6.

\begin{tabular}{|c|c|c|c|c|}
\hline \multicolumn{5}{|c|}{ SIMPLES } \\
\hline $\begin{array}{c}\text { Tamanho da } \\
\text { Amostra }\end{array}$ & $\boldsymbol{P}_{f}$ & $C . V$. da $P_{f}$ & $\operatorname{Erro}(\%)$ & $\begin{array}{l}\text { Tempo de } \\
\text { processamento } \\
(\text { min) }\end{array}$ \\
\hline 9.200 & $9,539 \times 10^{-5}$ & 0,2461 & 3,731 & 58 \\
\hline \multicolumn{5}{|c|}{ HIPERCUBO LATINO } \\
\hline $\begin{array}{c}\text { Tamanho da } \\
\text { Amostra }\end{array}$ & $P_{f}$ & C.V. da $P_{f}$ & $\operatorname{Erro}(\%)$ & $\begin{array}{c}\text { Tempo de } \\
\text { processamento } \\
(\text { min) }\end{array}$ \\
\hline 7.400 & $1,055 \times 10^{-4}$ & 0,2446 & 3,705 & 43 \\
\hline \multicolumn{5}{|c|}{ VARIÁVEIS ANTITÉTICAS } \\
\hline $\begin{array}{c}\text { Tamanho da } \\
\text { Amostra }\end{array}$ & $P_{f}$ & C.V.da $P_{f}$ & Erro $(\%)$ & $\begin{array}{c}\text { Tempo de } \\
\text { processamento } \\
(\text { min) }\end{array}$ \\
\hline 7.400 & $1,065 \times 10^{-4}$ & 0,2463 & 3,703 & 44 \\
\hline
\end{tabular}


Os resultados apresentados na Tabela 44 não são adotados no estudo comparativo, tal como apresentado na Tabela 43, pois o uso da Simulação de Subconjuntos com a Amostragem Simples precisa de uma quantidade maior de subconjuntos, diferentemente do uso da Amostragem por Hipercubo Latino e da Amostragem por Variáveis Antitéticas. Dessa forma, o tamanho total da amostra no uso da Amostragem Simples é de 9.200, enquanto que na Amostragem por Hipercubo Latino e na Amostragem por Variáveis Antitéticas é de 7.400. Mas apesar disso, este resultado é interessante pelo fato de apresentar um erro relativo menor. Um fato que merece destaque é a comparação entre a Simulação de Subconjuntos e o Monte Carlo Bruto, com referência ao tempo de processamento, ao coeficiente de variação e ao erro relativo. Pois, enquanto o Monte Carlo Bruto demorou aproximadamente 11 horas para realizar uma estimativa da probabilidade de falha com coeficiente de variação em torno de 0,25 e com erros relativos de 28,68\% (Amostragem Simples), 1,1\% (Amostragem por Hipercubo Latino) e 37,87\% (Amostragem por Variáveis Antitéticas), a Simulação de Subconjuntos demorou aproximadamente 45 minutos, para realizar uma estimativa da probabilidade de falha com coeficiente de variação em torno de 0,25 e com erros relativos em torno de 3,7 (Tabela 44).

\subsubsection{Tempo de processamento}

Os tempos de processamento, para uma amostra de tamanho 14.800, são apresentados na Tabela 45. Tais resultados também podem ser comparados de forma gráfica na Figura 120. Consideram-se o Monte Carlo Bruto (BRUTO), a Amostragem por Importância (AI), a Amostragem Assintótica (AA), a Amostragem Melhorada (AM) e a Simulação de Subconjuntos (SS). Utiliza-se a Amostragem Simples (Simples), a Amostragem por Hipercubo Latino (LHS) e a Amostragem por Variáveis Antitéticas (Antitéticas).

Tabela 45. Tempo de processamento para o Exemplo 6, para uma amostra de tamanho 14.800.

\begin{tabular}{|c|c|c|c|}
\hline & Simples & LHS & Antitéticas \\
\hline$\overline{\text { BRUTO }}$ & $1 \mathrm{~h} \mathrm{e} 37 \mathrm{~min}$ & $1 \mathrm{~h} \mathrm{e} 31 \mathrm{~min}$ & $1 \mathrm{~h} \mathrm{e} 38 \mathrm{~min}$ \\
\hline AI & $2 \mathrm{~h}$ & $2 \mathrm{~h} \mathrm{e} 2 \mathrm{~min}$ & $2 \mathrm{~h}$ e $2 \mathrm{~min}$ \\
\hline $\mathbf{A A}$ & $1 \mathrm{~h} \mathrm{e} 16 \mathrm{~min}$ & $1 \mathrm{~h} \mathrm{e} 15 \mathrm{~min}$ & $1 \mathrm{~h} \mathrm{e} 14 \mathrm{~min}$ \\
\hline $\mathbf{A M}$ & $1 \mathrm{~h} \mathrm{e} 27 \mathrm{~min}$ & $1 \mathrm{~h} \mathrm{e} 30 \mathrm{~min}$ & $1 \mathrm{~h} \mathrm{e} 30 \mathrm{~min}$ \\
\hline SS & $1 \mathrm{~h}$ e $52 \mathrm{~min}$ & $1 \mathrm{~h} \mathrm{e} 50 \mathrm{~min}$ & $1 \mathrm{~h}$ e $51 \mathrm{~min}$ \\
\hline
\end{tabular}

Observa-se que os tempos de processamentos são equivalentes, exceto para a técnica de Amostragem por Importância. Isso ocorre por causa das operações matemáticas que são inerentes à técnica, porém isto é compensado pelo fato de que esta técnica leva a bons resultados com uma amostra pequena. 


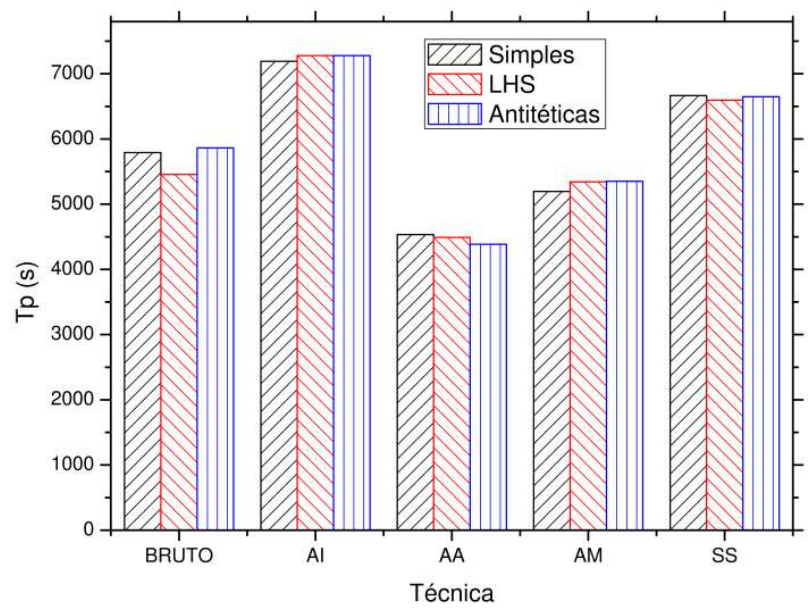

Figura 120. Comparativo dos tempos de processamento (em segundos) das tecnicas empregadas no Exemplo 6, para uma amostra de tamanho 14.800 . 


\section{CONSIDERAÇÕES FINAIS}

Nesta dissertação foram analisadas cinco técnicas utilizadas na estimativa da probabilidade de falha em confiabilidade de estruturas. Tais técnicas são as técnicas de simulação de Monte Carlo Bruto, a Amostragem por Importância usando Pontos de Projeto, a Amostragem Assintótica, a Amostragem Melhorada e a Simulação de Subconjuntos. Estas técnicas foram combinadas com três técnicas de amostragem básica, que são a Amostragem Simples, a Amostragem por Hipercubo Latino e a Amostragem por Variáveis Antitéticas. Dessa forma, são estudadas quinze combinações. Além disso, procurou-se analisar um amplo espectro de problemas que podem ser encontrados em confiabilidade de estruturas independente do tempo.

\subsection{Qualidade da resposta}

As técnicas estudadas neste trabalho apresentaram comportamentos bem característicos em cada problema. Em relação às técnicas de amostragem básica, foi verificado, de maneira geral, que a Amostragem por Hipercubo Latino e a Amostragem por Variáveis Antitéticas levaram a Amostragem por Importância, a Amostragem Assintótica, a Amostragem Melhorada e a Simulação de Subconjuntos, a apresentarem resultados melhores, em termos do valor médio da probabilidade de falha, em comparação com o uso da Amostragem Simples. Por outro lado, a Simulação do Monte Carlo Bruto não apresenta melhoras significativas, devido ao uso da Amostragem por Hipercubo Latino e da Amostragem por Variáveis Antitéticas.

A Amostragem por Importância é a que apresenta uma convergência mais rápida, em comparação com as outras técnicas estudadas, nos casos em que ela pode ser aplicada. Porém, como visto no Exemplo 4, ela apresentou um resultado tendencioso.

A Amostragem Assintótica consegue apresentar uma boa convergência na maioria dos casos estudados nesta dissertação, além disso, esta técnica necessita que uma amostra diferente seja gerada para cada ponto de suporte. Dessa forma, quando se desejar utilizar uma quantidade pequena de pontos amostrais para se estimar a probabilidade de falha, uma amostra menor ainda deverá ser gerada para cada ponto de suporte, isso visa não comprometer o tempo de processamento. Além do mais, o aumento do desvio padrão em cada ponto de 
suporte aumenta a dispersão da amostra o que pode aumentar a incerteza da estimativa da probabilidade de falha.

A Amostragem Melhorada contorna o efeito do aumento da dispersão da amostra em cada ponto de suporte, tal como visto na Amostragem Assintótica. Isso acontece devido ao fato de a Amostragem Melhorada parametrizar a equação de estado limite, ao invés de parametrizar as variáveis aleatórias do problema. Assim, está técnica permite utilizar uma amostra maior em cada ponto de suporte, uma vez que a amostra é a mesma para todos pontos de suporte.

A Simulação de Subconjuntos é uma técnica que se mostrou bastante vantajosa na estimativa da probabilidade de falha em diversos tipos de problemas. Esta técnica permite que se faça uma boa estimativa de tal probabilidade, com uma amostra pequena. A Simulação de Subconjuntos só não foi superior à Amostragem por Importância, nos problemas em que a Amostragem por Importância apresentou bons resultados ou pode ser aplicada.

\subsection{Tempo de processamento}

Em relação ao tempo de processamento, verificou-se que a técnica de Amostragem por Importância apresenta um tempo de processamento maior que as outras técnicas, para uma amostra de mesmo tamanho. Por outro lado, isto é recompensado pela rápida convergência da técnica. $\mathrm{Na}$ análise de um sistema, a técnica que apresenta prejuízo é a Amostragem Melhorada, devido à necessidade de se avaliar a parametrização de cada componente do sistema. A Simulação de Subconjuntos apresenta uma relação não linear entre o tempo de processamento e o tamanho da amostra. Porém, isto é compensado pela possibilidade de utilizar uma amostra pequena na estimativa da probabilidade de falha, sendo este um dos principais atrativos da técnica.

\subsection{Sugestões para trabalhos futuros}

Alguns aprimoramentos deste estudo podem ser realizados em outros trabalhos, os destaques são:

- Estudar a otimização dos parâmetros de entrada de cada técnica;

- Aplicar as técnicas implementadas no StRAnD em conjunto com as técnicas de superfície de resposta;

- Aplicação de técnicas de Quase-Monte Carlo; 
- Estudo de outras técnicas de Amostragem;

- Estudo comparativo na aplicação em problemas de dinâmica estocástica linear e não linear;

- Aplicação de programação em paralelo;

- Desenvolvimento de um programa de Elementos Finitos parametrizável para aplicação em Confiabilidade de Estruturas. 


\section{REFERÊNCIAS}

ABNT - ASSOCIAÇÃO BRASILEIRA DE NORMAS TÉCNICAS. NBR 6123: Forças devidas ao vento em edificações. Rio de Janeiro, 1988.

ANG, A. H-S.; TANG, W. H. Probability Concepts in Engineering: Emphasis on Applications to Civil and Environmental Engineering, 2nd Edition. New York: John Wiley \& Sons, 1984.

AU, S-K. Reliability-based design sensitivity by efficient simulation. Computer and structures, v.83, p. 1048-1061, 2005.

AU, S-K.; BECK, J. L. Estimation of small failure probabilities in high dimensions by subset simulation. Probabilistic engineering mechanics, v.16, p. 263-277, 2001.

AU, S-K.; CHING, J.; BECK, J. L. Application of subset simulation methods to reliability benchmark problems. Structural safety, v.29, p. 183-193, 2007.

BECK, A. T. Curso de confiabilidade estrutural: notas de aula. São Carlos: EESC-USP, 2012.

BUCHER, C. Asymptotic sampling for high-dimensional reliability analysis. Probabilistic engineering mechanics, v.24, p.504-510, mar. 2009.

CODA, H. B.; GRECO, M. A simple FEM formulation for large deflection 2D frame analysis based on position description. Computer Methods in Applied Mechanics and Engineering, v. 193, p. 3541-3557, 2004.

CODA, H. B.; PACCOLA, R. R. An alternative positional FEM formulation for geometrically non-linear analysis of shells: curved triangular isoparametric elements, Computational Mechanics, v. 40, p. 185-200, 2007.

CODA, H. B.; PACCOLA, R. R. Improved finite element for 3D laminate frame analysis including warping for any cross-section, Applied Mathematical Modelling, v.34, pp. 11071137, 2010.

CODA, H. B.; PACCOLA, R. R. A FEM procedure based on positions and unconstrained vectors applied to non-linear dynamic of 3D frames, Finite Elements in Analysis and Design, v.47, p. 319-333, 2011.

DITLEVSEN, O. Principle of normal tail approximation, Journal of Engineering Mechanics Division, v. 107, n. 6, p. 1191-1208, 1981.

GATTAS, M. Método de Levenberg-Marquardt: notas de aula. Rio de Janeiro: PUC-Rio. 
GOMES, W. J. S.; BECK, A. T. Global structural optimization considering expected consequences of failure and using ANN surrogates, Computers and Structures, v.126, p.56$68,2013$.

GRECO, M.; CODA, H. B. Positional FEM Formulation for flexible multi-body dynamics analysis, Journal of Sound and Vibration, v.290, p. 1141-1174, 2006.

GRECO, M.; GESUALDO, F. A. R.; VENTURINI, W. S.; CODA, H. B. Nonlinear positional formulation for space truss analysis, Finite Elements in Analysis and Design, v. 42, p. 1079-1086, 2006.

HANSEN, S. R.; VANDERPLAATS, G.N. An approximation method for configuration optimization of trusses, American Institute of Aeronautics and Astronautics Journal, v.28, n.1, p.161-172, 1990.[

HAMMERSLEY, J. M.; MORTON, K. W. A new Monte Carlo technique: antithetic variates, Mathematical proceedings of the Cambridge Philosophical Society , v. 52, n. 3, p. 449475, 1956.

HASTINGS, W. K. Monte Carlo sampling methods using Markov Chain and their applications. Biometrika, v.57, n.1, p. 97-109,1970.

HURTADO, J.E.; BARBAT, A.H. Monte Carlo Techniques in Computational Stochastic Mechanics. Archives of Computational Methods in Engineering State of the art reviews, v.5, n.1, p.3-30, 1998.

L'ECUYER. Uniform random number generators: A review. In: WINTER SIMULATION CONFERENCE, 1997.

McKAY, M. D.; BECKMAN, R. J.; CONOVER, W. J. A comparison of three methods for selecting values of input variables in the analysis of output from a computer code. Technometrics, v.21, n.2, p. 239-124, mai. 1979.

MELCHERS, R. E.; AHAMMED, M. A fast approximate method for parameter sensitivity estimation in Monte Carlo structural reliability. Computers and Structures, v. 82, p. 55-61, 2004.

METROPOLIS, N.; ULAM, S. The Monte Carlo method. Journal of the american statistical association, v.44, n.247, p. 335-341, set. 1949.

METROPOLIS, N. et al. Equation of state calculation by fast computing machines. The journal of chemical physics, v.21, n.6, p. 1087-1092, jun. 1953.

MOntegomery, D. C.; RUNGER, G. C. Applied Statistics and Probability for Engineers. John Wiley. 2003. 
NAESS, A.; LEIRA, B. J.; BATSEVYCH, O. System reliability analysis by enhanced Monte Carlo simulation. Structural safety, v.31, p. 349-355, 2009.

NOGUEIRA, F. M. A. Cadeias de Markov: notas de aula: Juiz de Fora, UFJF, 2009.

OLSSON, A.; SANDEBERG, G.; DAHLBLOM, O. On latin hypercube sampling for structural reliability analysis. Structural safety, v.25, p. 47-68, 2003.

ROBERT, C.; CASELlA, G. A short history of Markov Chain Monte Carlo: Subjective recollections from incomplete data. Statistical science, v.0, n.0, p. 1-14, fev. 2011.

SCHUËLLER, G. I.; PRANDLWARTER, H. J. Benchmark study on reliability estimation in higher dimensions of structural systems - An overview. Structural Safety v.29, p. 167-182, 2007.

SICHANI, M. T.; NIELSEN, S. R. K.; BUCHER, C. Applications of asymptotic sampling on high dimensional structural dynamic problems. Structural safety, v.33, p. 305-316, 2011a.

SICHANI, M. T.; NIELSEN, S. R. K.; BUCHER, C. Efficient estimation of first passage probability of high-dimensional nonlinear systems. Probabilistic engineering mechanics, v.26, p.539-549, mar. 2011b.

SICHANI, M. T.; NIELSEN, S. R. K.; BUCHER, C. Closure to "Applications of asymptotic sampling on high dimensional structural dynamic problems". Structural safety, v.46, p. 11$12,2014$.

TANG, Z.; LU, Z; PAN, W. Discussion on: Applications of asymptotic sampling on high dimensional structural dynamic problems: M. T. SICHANI, S. R. K. NIELSEN, C. BUCHER, 33(2011) 305-316. Structural safety, v.46, p. 8-10, 2014.

VERZENHASSI, C, C. Otimização de risco estrutural baseada em confiabilidade. Dissertação (Mestrado), Escola de Engenharia de São Carlos, Universidade de São Paulo, São Carlos, 2008.

VIEIRA, C. E. C.; RIBEIRO, C. C.; SOUZA, R. C. Geradores de números aleatórios. 2004, 81p. Monografia (Ciência da Computação) - Pontifícia Universidade Católica do Rio de Janeiro, Rio de Janeiro, 2004.

ZUEV, K. Random walks in high dimensions: Metropolis algorithm and its modifications. notas de aula. Hong-Kong: University of Science and Technology, 2010.

ZUEV, K.; BECK, J.L.; AU, S. K.; KATAFYGIOTIS, L.S. Bayesian post-processor and other enhancements of Subset Simulation for estimating failure probabilities in high dimensions. Computers and Structures, v.92-93, 283-296, 2012. 


\section{APÊNDICE: StRAnD - Manual de Usuário}

O StRAnD (Structural Risk Analisys and Design) é um programa especializado em análise de risco e é desenvolvido junto ao departamento de Engenharia de Estruturas da Escola de Engenharia de São Carlos da Universidade de São Paulo (EESC-USP). Atualmente o programa é capaz de realizar análise de confiabilidade dependente e independente do tempo, confiabilidade de sistemas e otimização de risco. Além disso, ele possui uma interface de acoplamento com programas de elementos finitos acadêmico, desenvolvido na EESC-USP, (Acadframe) e comercial (ANSYS). Neste trabalho será utilizado o Acadframe, uma vez que a interface entre ambos os programas leva a um menor tempo de processamento, uma vez que a necessidade de escrever em arquivo de texto, a cada simulação, é eliminada, tal como é necessário no uso do ANSYS.

Na Figura 121 é apresentado um fluxograma que descreve o trecho implementado no StRAnD e a relação entre as diversas partes do programa.

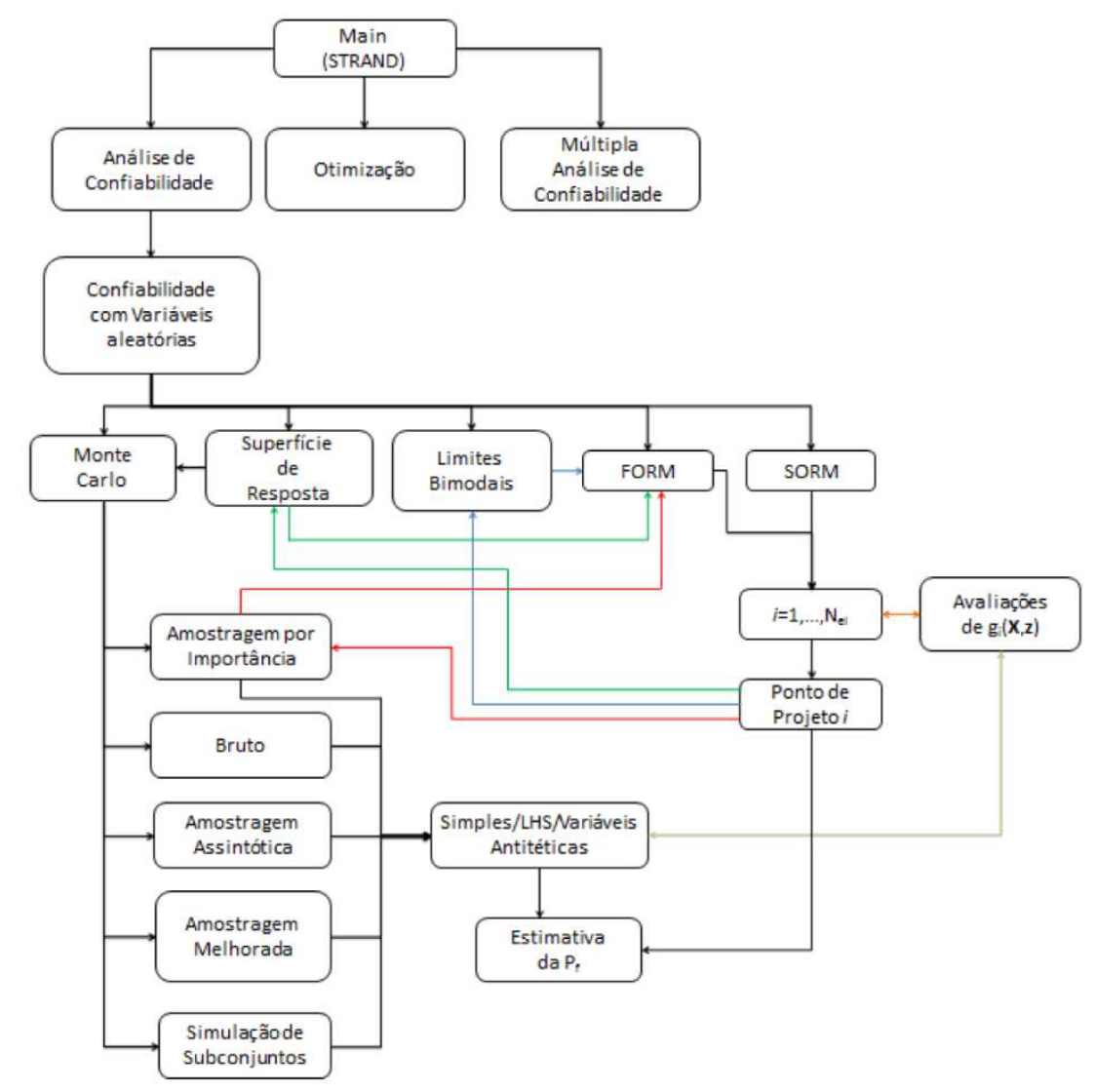

Figura 121. Diagrama básico do StRAnD. 


\section{Entrada de dados no StRAnD}

A entrada de dados do StRAnD é realizada via arquivo de texto. Da mesma forma, o programa de elementos finitos que está acoplado ao StRAnD, o Acadframe, também possui entrada de dados via arquivo de texto. O arquivo de entrada do StRAnD possui o nome STRAND_INPUT.TXT e o do Acadframe ACADFRAME_INPUT.TXT. No arquivo STRAND_INPUT.TXT o usuário é capaz de definir o tipo de problema, o tipo de análise a ser realizada, as variáveis aleatórias e todas as variáveis responsáveis pela execução da análise de confiabilidade que se deseja realizar. Um trecho de um arquivo de entrada de dados pode ser vista a seguir.

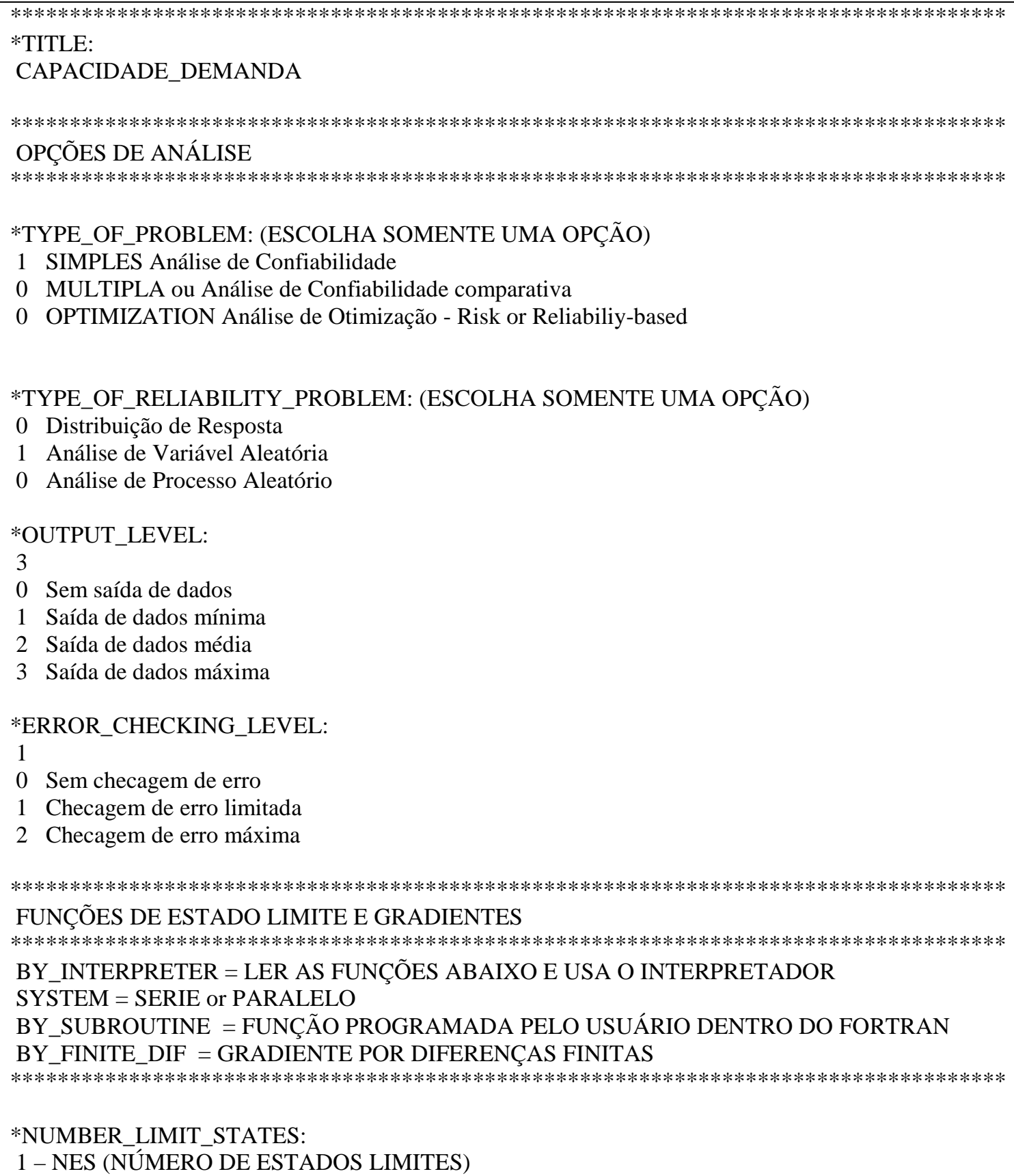




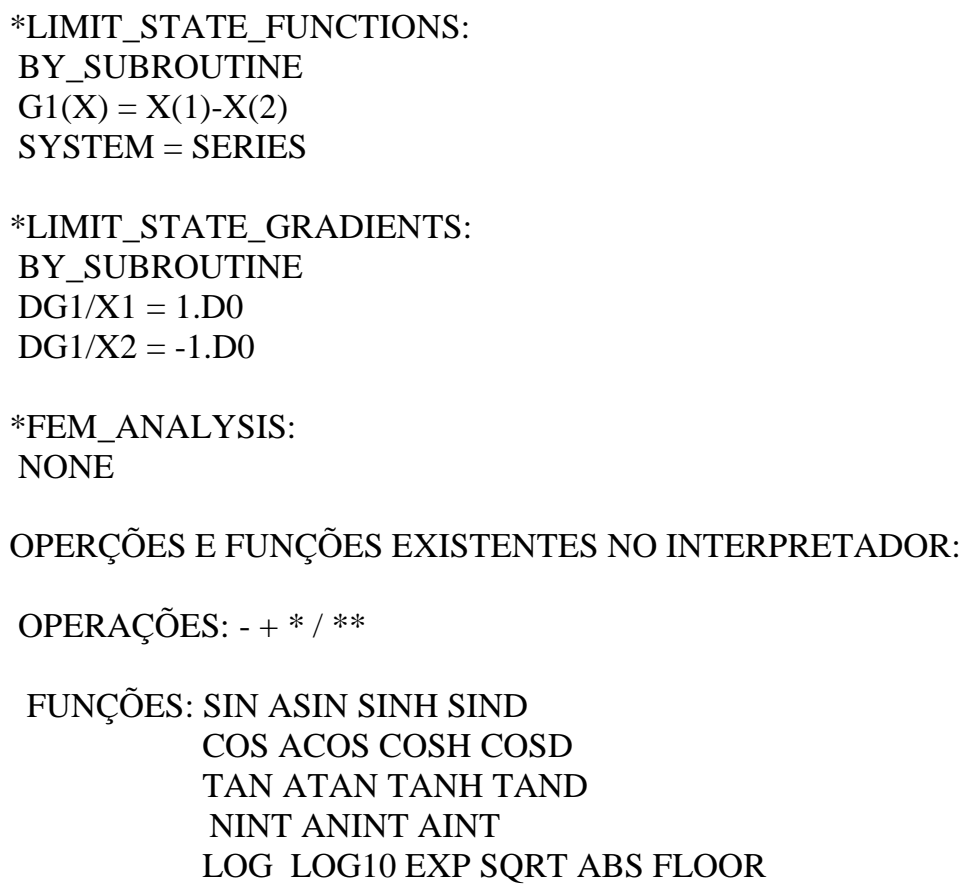

P:M:C: - CAPS LOCK - Permite: Parâmetros (P), Momentos (M) or C.V. (C) to be specified

Distribution codes:

$$
\begin{aligned}
& 0 \text { - Determinística } \\
& 1 \text { - Uniforme } \\
& 2 \text { - Normal } \\
& 3 \text { - Lognormal } \\
& 4 \text { - Exponencial } \\
& 5 \text { - Raleigh } \\
& 6 \text { - Logistica } \\
& 7 \text { - Gumbel para mínimos } \\
& 8 \text { - Gumbel para máximos } \\
& 9 \text { - Frechet para minimos } \\
& 10 \text { - Frechet para maximos } \\
& 11 \text { - Weibull para minimos } \\
& 12 \text { - Weibull para maximos } \\
& 13 \text { - Normal Truncada } \\
& 14 \text { - Gamma } \\
& 15 \text { - Beta }
\end{aligned}
$$




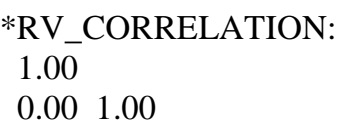

1 Monte Carlo Bruto

1 Amostragem por Importância usando os pontos de projeto

1 Amostragem Assintótica

1 Amostragem Melhorada

1 Simulação de Subconjuntos

*BASIC_SAMPLES_GENERATION:

1 Amostragem Simples

1 Hipercubo Latino

1 Variáveis Antitéticas

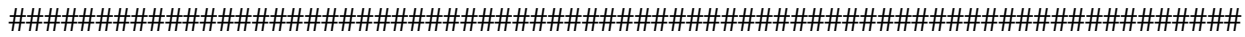

********************** NAESS TECHNIQUES $* * * * * * * * * * * * * * * * * * * * * * * * * * * * * * * *$

*ENHANCED_SIM_OPTIONS:

69000 Tamanho da Amostra

1111 Semente do gerador de números aleatórios

100 Números de pontos de suporte

0.4 Valor inicial de lambda

0.9 Valor final de lambda

0.95 Nível de confiança

0 Gráfico de regressão $(\mathrm{S}$ ou $\mathrm{N})$ ?

0 Gráfico de convergência $(\mathrm{S}$ ou $\mathrm{N})$ ?

10 Se quer gráfico de convergência, defina o número de pontos.

100 Se quer gráfico de convergência defina o valor inicial do tamanho da amostra

************************** ASYMPTOTIC SAMPLING $* * * * * * * * * * * * * * * * * * * * * * * * * * * * * *$

\section{*AS_SIM_OPTIONS:}

13800 Tamanho da Amostra em cada ponto de suporte

1111 Semente do gerador de números aleatórios

5 Números de pontos de suporte

2 Número de parâmetros na função de regressão $\left(2: A+B /\left(x^{\wedge} 2\right) ; 3: A+B /\left(x^{\wedge} C\right)\right)$

0.4 Valor inicial de $\mathrm{f}$

0.6 Valor final de $\mathrm{f}$

0 Busca adaptativa pelo interval de $\mathrm{f}(\mathrm{S}$ ou $\mathrm{N})$ ?

0 Achar limite superior e inferior de $\mathrm{f}(1)$, ou somente superior (0) (se usando busca adaptativa)

10 Número de falha mínimo por ponto de suporte

0.95 Nível de confiança

0 Gráfico de regressão $(\mathrm{S}$ ou $\mathrm{N})$ ?

0 Gráfico de convergência ( $\mathrm{S}$ ou $\mathrm{N})$ ?

10 Se quer gráfico de convergência, defina o número de pontos.

100 Se quer gráfico de convergência defina o valor inicial do tamanho da amostra 


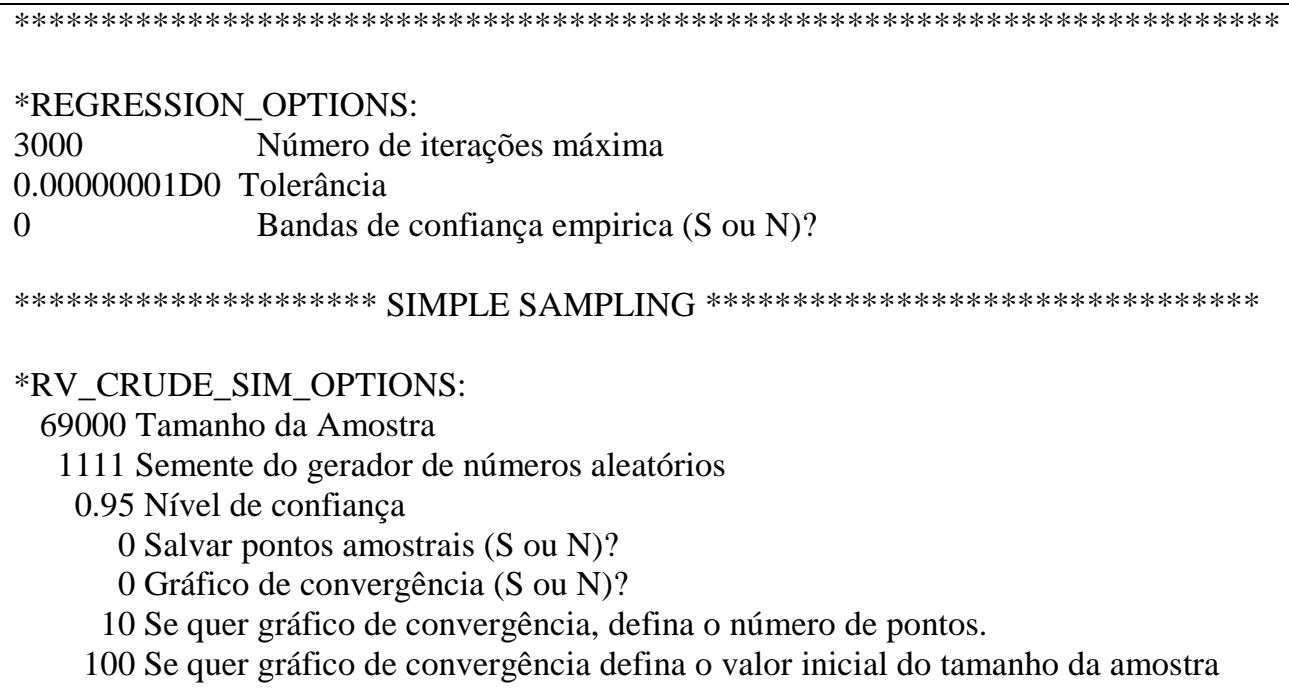

15000 Tamanho da Amostra em cada subconjunto

1111 Semente do gerador de números aleatórios

7 Número máximo de subconjuntos

0.1 probabilidade de falha condicional

0.95 Nível de confiança

0 Salvar pontos amostrais ( $\mathrm{S}$ ou $\mathrm{N})$ ?

0 Gráfico de convergência ( $\mathrm{S}$ ou $\mathrm{N})$ ?

10 Se quer gráfico de convergência, defina o número de pontos.

100 Se quer gráfico de convergência defina o incremento da amostra em cada subconjunto

*RW_OPTIONS:

1 parâmetros definidos pelo usuário (1) ou multiplique os desvios padrões originais por alfa (0) 3.0D0 alfa

*RW_DATA:
$1 \quad \mathrm{M} \quad 0.0$
$0.140 \quad 0.0$
0.0

$\begin{array}{llllll}1 & \mathrm{M} & 0.0 & 0.105 & 0.0 & 0.0\end{array}$

$* * * * * * * * * * * * * * * * * * * * * * * * * * * * * * * * * * * * * * * * * * * * * * * * * * * * * * * * * * * * * * * * * * * * * * * * *$

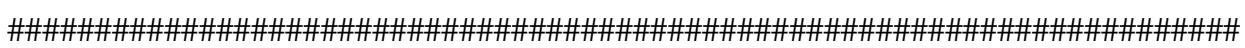

Os passos para entrada dos dados das simulações de Monte Carlo são apresentados a seguir: 
No uso de equações de estado limite que dependem da resposta de um modelo de elementos finitos se utiliza a flag “*FEM_ANALYSIS:”, onde as opções são: “ACADFRAME”, para utilizar o programa Acadframe, “ANSYS”, se for desejável utilizar o ANSYS e "NONE" quando não se deseja realizar análise de elementos finitos.

*FEM_ANALYSIS:

ACADFRAME

Através da flag “*NUMBER_RV:”, se define o número de variáveis aleatórias do problema.

*NUMBER_RV:

2 - NRV

Para definir os parâmetros das variáveis aleatórias se utiliza a flag “*RV_DATA:”, onde a sequência de inserção dos dados para cada variável aleatória do problema é feito por cada linha. Desta forma em cada linha define-se o código do tipo de distribuição de probabilidade; a forma de inserção do dado, se é por parâmetros (P), momentos (M) ou coeficiente de variação (C). Em seguida são inseridos os dados de acordo com a instrução descrita logo abaixo.

*RV_DATA:

$\begin{array}{llllll}2 & \mathrm{M} & 2.0 & 0.20 & 0.0 & 0.0 \\ 2 & \mathrm{M} & 1.0 & 0.15 & 0.0 & 0.0\end{array}$

A flag “*RW_OPTIONS:" é responsável por definir se o usuário irá definir os desviospadrão do caminhante aleatório de maneira direta ou como uma parcela dos desvios-padrão do problema original.

*RW_OPTIONS:

1 Se definido pelo usuário escolha (1) ou multiplicando o original por alfa (0) 3.0D0 alfa

O mesmo procedimento adotado na definição das variáveis aleatórias deve ser realizado para a definição do caminhante aleatório utilizado na simulação de subconjuntos, isso é feito utilizando a flag “*RW_DATA:”. Neste caso deve-se manter a média com valor zero e é aconselhável utilizar uma distribuição de probabilidades uniforme ou normal.

$\begin{array}{ccllll}* \text { RW_DATA: } & & & & \\ 1 & \mathrm{M} & 0.0 & 0.06 & 0.0 & 0.0 \\ 1 & \mathrm{M} & 0.0 & 0.045 & 0.0 & 0.0\end{array}$


Em STRAND_INPUT.TXT é realizada a escolha das técnicas em cada análise e suas combinações, tal como apresentado a seguir:

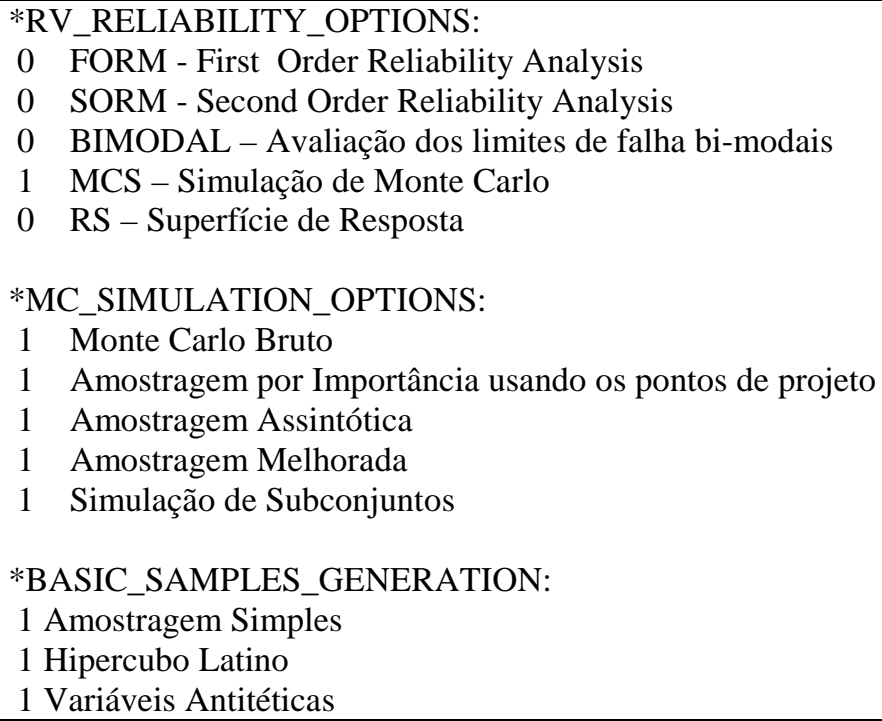

Quando o usuário escolhe o valor 1 para a variável MCS, as opções de “*MCSIMULATION_OPTIONS:", são ativadas. Assim pode-se escolher entre realizar o Monte Carlo Bruto, Amostragem por Importância utilizando pontos de projeto, Amostragem Assintótica, Amostragem Melhorada e Amostragem por Subconjunto. Através da opção “*BASIC_SAMPLES_GENERATION:”, é realizada a escolha da amostragem básica, neste caso as opções são: Amostragem Simples, Amostragem por Hipercubo Latino e Variáveis Antitéticas.

O StRAnD atualmente apresenta 15 possibilidades para simulação de Monte Carlo. Tais combinações são:

○ Monte Carlo Bruto com:

- Amostragem Simples (SIMC)

- Amostragem por Hipercubo Latino (SLHS)

- Amostragem por Variáveis Antitéticas (ASIMC)

- Amostragem por Importância com:

- Amostragem Simples (ISMC)

- Amostragem por Hipercubo Latino (ILHS)

- Amostragem por Variáveis Antitéticas (AISMC)

- Amostragem Assintótica com:

- Amostragem Simples (ASMC)

- Amostragem por Hipercubo Latino (ALHS) 
- Amostragem por Variáveis Antitéticas (AASMC)

○ Amostragem Melhorada com:

- Amostragem Simples (AM)

- Amostragem por Hipercubo Latino (AM_LHS)

- Amostragem por Variáveis Antitéticas (AAM)

○ Simulação de Subconjuntos com:

- Amostragem Simples (SS)

- Amostragem por Hipercubo Latino (SSLHS)

- Amostragem por Variáveis Antitéticas (SSA)

Além das 15 combinações apresentadas acima, o StRAnD possui rotinas de criação de superfície de respostas, o que permite a utilização destas em Simulação de Monte Carlo. Qualquer uma das técnicas de simulação acima poderá ser combinada com o uso de superfícies de resposta. Por ora, o StRAnD permite a construção de superfícies de resposta polinomiais de primeira e segunda ordem. Há planos para implementar técnicas modernas de superfície de resposta, envolvendo redes neurais artificiais, krigagem, etc. Neste trabalho não serão abordados exemplos com uso de superfície de resposta. Em sua versão atual, o StRAnD aplica a técnica de superfície de respostas ao Monte Carlo Bruto e à Amostragem por Importância. Desta forma, os usuários do StRAnD terão mais opções de simulação, que poderão viabilizar a resolução de problemas complexos.

\section{Saída de dados do StRAnD}

Neste capítulo serão apresentadas algumas novas funcionalidades do StRAnD, em termos de saída de dados. Diferentes análises, em relação a qualidade da simulação de Monte Carlo, poderão ser efetuadas.

O arquivo principal de saída de dados é o STRAND_OUTPUT.TXT, ele contém o relatório final, contendo os resultados finais das simulações. Acrescentou-se a este o arquivo FULL_OUTPUT.TXT, que contêm todas as realizações das variáveis aleatórias utilizadas na simulação. A impressão do arquivo é feita como uma matriz, onde o número de linhas é o número de realizações e o número de colunas é igual ao número de variáveis aleatórias. Um trecho de um arquivo FULL_OUTPUT, pode ser observado abaixo: 


\section{Exemplo: STRAND_SIMC_FULL_OUTPUT.TXT (Monte Carlo Simples)}

$\begin{array}{llllllll}27.873 & 22.524 & 34.478 & 26.015 & 26.449 & 29.886 & 37.712 & 25.363 \\ 26.688 & 23.428 & 30.666 & 27.689 & 27.447 & 25.774 & 21.167 & 27.289 \\ 27.082 & 36.160 & 18.133 & 26.611 & 25.854 & 32.355 & 28.312 & 28.984 \\ 27.286 & 22.246 & 26.399 & 31.949 & 28.995 & 17.029 & 32.271 & 24.613 \\ 32.332 & 29.822 & 24.849 & 21.885 & 20.671 & 25.558 & 26.407 & 35.620 \\ 23.712 & 34.848 & 25.895 & 35.313 & 31.330 & 29.286 & 34.697 & 27.467\end{array}$

Com estes dados e utilizando um gerador gráfico a escolha do usuário, pode-se gerar os gráficos do comportamento conjunto de quaisquer duplas ou triplas de variáveis aleatórias, tal como na Figura 122(a) e na Figura 122(b), respectivamente.

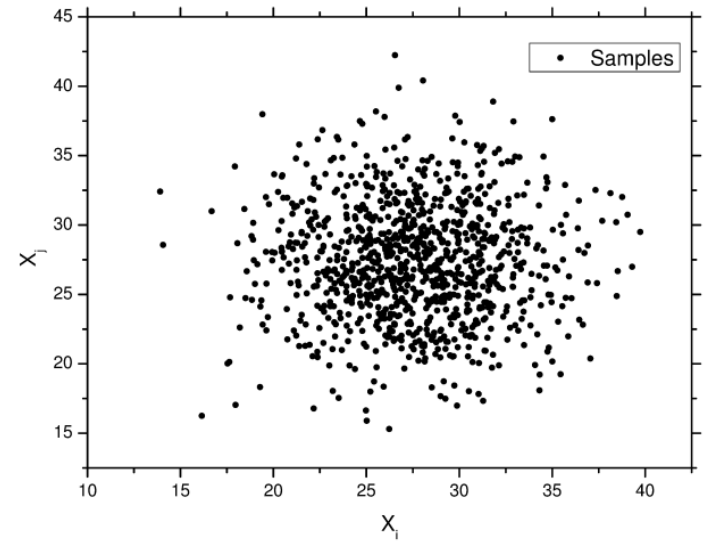

a)

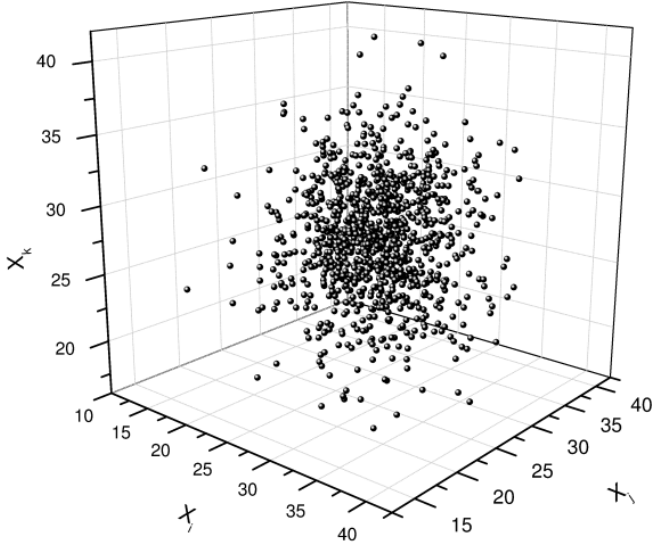

b)

Figura 122. Dado de saída do StRAnD: Amostra aleatória a) 2-D e b) 3-D.

A Simulação de Subconjuntos possui uma saída de dados diferenciada, uma vez que cada arquivo FULL_OUTPUT salva os dados de um subconjunto. Desta forma o arquivo saí com o seguinte nome:

\section{STRAND_X_SUBSET_FULL_OUTPUT.TXT (Simulação de Subconjunto)}

onde no lugar de $\mathrm{X}$ deve sair o número do subconjunto simulado, por exemplo: STRAND_1_SUBSET_FULL_OUTPUT.TXT, para o primeiro subconjunto.

É interessante a aplicação deste tipo de arquivo quando se utiliza a Simulação de Subconjuntos, uma vez que a geração de cada subconjunto pode ser facilmente notada quando se gera os gráficos do comportamento conjuntos de pares de variáveis aleatórias. Também se pode observar a qualidade das amostras condicionais, uma vez que o desvio padrão do caminhante aleatório interfere no nível de aceitação e rejeição destas. Um exemplo do que se pode obter, quando se utiliza Simulação por Subconjuntos, é observada da Figura 123, onde se observa a geração dos diferentes subconjuntos para o problema apresentado no Exemplo 1. 


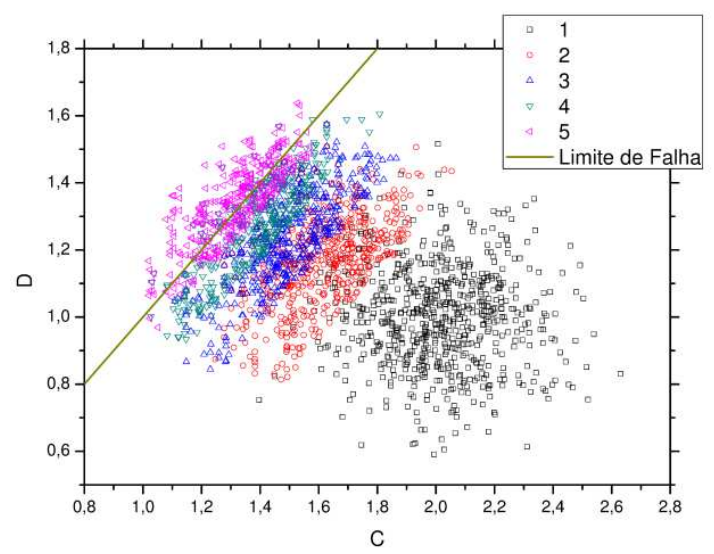

Figura 123. Dado de saída do StRAnD: geração de subconjuntos na simulação de subconjuntos, no Exemplo 1.

Os arquivos CONVERGENCE_OUTPUT.TXT contêm os dados de convergência da probabilidade de falha com o número de simulações.

Os dados são apresentados na seguinte ordem:

- Coluna 1: Numero de simulações por cada passo de convergência;

- Coluna 2: Coeficiente de variação da probabilidade de falha;

- Coluna 3: Limite inferior do intervalo de confiança da probabilidade de falha;

- Coluna 4: Probabilidade de falha;

- Coluna 5: Limite superior do intervalo de confiança da probabilidade de falha;

- Coluna 6: Limite inferior do intervalo de confiança do índice de confiabilidade;

- Coluna 7: Índice de confiabilidade;

- Coluna 8: Limite superior do intervalo de confiança do índice de confiabilidade;

Um trecho de um arquivo do tipo CONVERGENCE_OUTPUT.TXT pode ser observado abaixo:

Exemplo: STRAND_SIMC_CONVERGENCE_OUTPUT.TXT (Monte Carlo Simples)

\begin{tabular}{|c|c|c|c|c|c|c|c|}
\hline $\mathrm{N}$ & cov & PF_LOWER & PF & PF_UPPER & BETA_LOWER & BETA & BETA_UPPER \\
\hline 100000 & 0.5773444956 & 0.00000000000 & 0.0000300000 & 0.0000639472 & 3.8304781988 & 4.0128108111 & 38.1837661841 \\
\hline 200000 & 0.4472091233 & 0.0000030872 & 0.0000250000 & 0.0000469128 & 3.9060278866 & 4.0556269811 & 4.5203306757 \\
\hline 300000 & 0.4472106141 & 0.0000020581 & 0.0000166667 & 0.0000312753 & 4.0029763520 & 4.1494099843 & 4.6054317296 \\
\hline 400000 & 0.3535502970 & 0.0000061411 & 0.0000200000 & 0.0000338589 & 3.9841649191 & 4.1074796546 & 4.3725180474 \\
\hline
\end{tabular}

A partir dos dados apresentados anteriormente e utilizando um programa adequado para a geração de gráficos, pode-se obter gráficos de convergência da probabilidade de falha $P_{f}$ (ou do índice de confiabilidade $\beta$ ), tal como na Figura 124. 


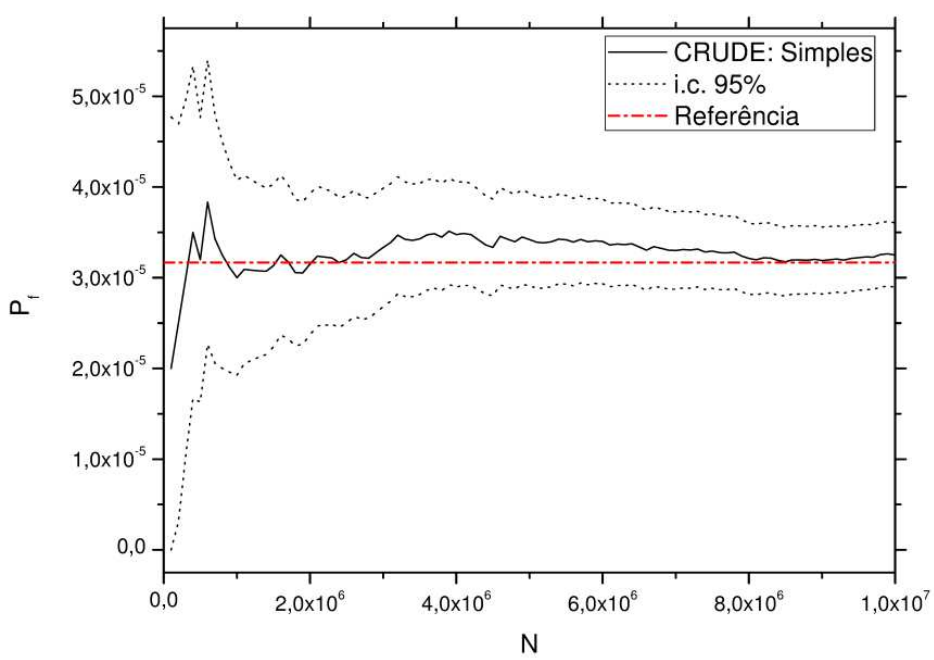

Figura 124. Dado de saída do StRAnD: Graficos de convergência, no Exemplo 1.

Os arquivos REGRESSION_OUTPUT.TXT contêm os dados utilizados para a plotagem dos gráficos de regressão não linear. As técnicas que possuem essa opção de saída de dados são: Amostragem Assintótica e Amostragem Melhorada. Neste tipo de arquivo, a sequência de impressão é:

- Coluna 1: Valor do parâmetro $f$;

- Coluna 2: Valor da banda de confiança inferior do ajuste;

- Coluna 3: Valores de $\beta / f$ obtidas para qualquer ponto da função ajustada, que é contínua;

- Coluna 4: Valor da banda de confiança superior do ajuste;

- Coluna 5: Valor do parâmetro $f$, para os pontos de suporte;

- Coluna 6: Valores de $\beta / f$ obtidas para os pontos de suporte;

Um trecho de um arquivo do tipo REGRESSION_OUTPUT, pode ser visto abaixo:

Exemplo: STRAND_ASMC_REGRESSION_OUTPUT.TXT (Amostragem Assintótica)

\begin{tabular}{|c|c|c|c|c|c|}
\hline$F$ & CONF_BAND_LOWER & BETA/F & CONF_BAND_UPPER & F_SUPPORT & BETA_SUPPORT \\
\hline 0.0100000000 & -5883.0895851943 & -5714.8613699909 & $-5546.6331 \overline{5} 47874$ & $0.80 \overline{0} 0000000$ & $3.71 \overline{7} 4283363$ \\
\hline 0.0200000000 & -1467.2879382505 & -1425.2728568130 & -1383.2577753754 & 0.7000000000 & 3.4157939797 \\
\hline 0.0300000000 & -649.5469030899 & -630.9046136319 & -612.2623241739 & 0.6000000000 & 2.9951639968 \\
\hline 0.0400000000 & -363.3375533317 & -352.8757285185 & -342.4139037053 & 0.5000000000 & 2.2806064245 \\
\hline 0.0500000000 & -230.8635253075 & -224.1880731231 & -217.5126209388 & 0.4000000000 & 1.0288384635 \\
\hline 0.0600000000 & -158.9023397417 & -154.2836677232 & -149.6649957048 & & \\
\hline 0.0700000000 & -115.5120301301 & -112.1335160442 & -108.7550019583 & & \\
\hline 0.0800000000 & -87.3500666630 & -84.7764464449 & -82.2028262267 & & \\
\hline 0.0900000000 & -68.0423341909 & -66.0205295920 & -63.9987249930 & & \\
\hline 0.1000000000 & -54.2316443096 & -52.6045325960 & -50.9774208825 & & \\
\hline
\end{tabular}

Com os dados obtidos nos arquivos REGRESSION_OUTPUT, pode-se gerar gráficos dos pontos de suporte, da função ajustada e das bandas de confiança, tal como na Figura 125. 


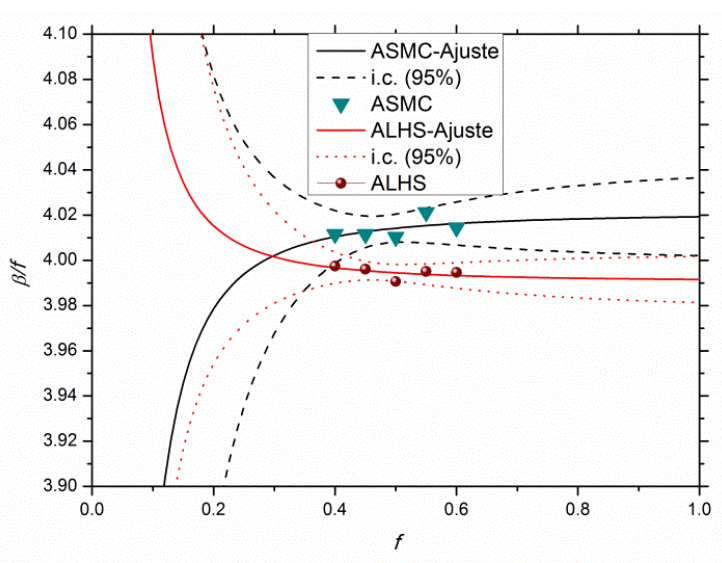

Figura 125. Dado de saída do StRAnD: Graficos de regressão utilizando o modelo do Exemplo 1.

Os arquivos RANK_OUTPUT contêm a posição que cada uma das realizações das variáveis aleatórias possui, caso estas fossem colocadas em ordem decrescente. Este conjunto de dados é utilizado na verificação da qualidade do Hipercubo Latino, porém seu uso deve ser feito com atenção, uma vez que o algoritmo de ordenamento, que foi adicionado, pode ser computacionalmente custoso. A impressão do arquivo é feita como uma matriz, onde o número de linhas é o número de realizações e o número de colunas é igual ao número de variáveis aleatórias. A técnica que faz uso desta saída de dados é a de Monte Carlo Padrão com Hipercubo Latino. Um trecho de um arquivo deste tipo pode ser verificado abaixo:

Exemplo: STRAND_SLHS_RANK_OUTPUT.TXT (Hipercubo Latino)

$\begin{array}{lllllllll}2949.0 & 2196.0 & 1389.0 & 4238.0 & 1869.0 & 2745.0 & 3693.0 & 4996.0 & 2492.0 \\ 1554.0 & 235.00 & 4013.0 & 420.00 & 4761.0 & 4887.0 & 2952.0 & 2281.0 & 3373.0 \\ 3410.0 & 2414.0 & 1669.0 & 2027.0 & 4478.0 & 876.00 & 4881.0 & 4966.0 & 2227.0 \\ 1789.0 & 4603.0 & 4278.0 & 3893.0 & 1690.0 & 4128.0 & 1807.0 & 4190.0 & 4854.0 \\ 4474.0 & 4319.0 & 4535.0 & 4533.0 & 4009.0 & 295.00 & 503.0 & 1650.0 & 2021.0 \\ 4538.0 & 3814.0 & 795.00 & 3575.0 & 2758.0 & 89.000 & 2075.0 & 1721.0 & 3815.0\end{array}$

Como dito anteriormente, através do arquivo RANK_OUTPUT, que é utilizado apenas no Método de Monte Carlo Padrão com Amostragem por Hipercubo Latino, pode-se verificar a qualidade do hipercubo latino gerado, assim através dos dados do arquivo, pode-se gerar a Figura 126, que representa o comportamento da amostragem para duas variáveis aleatórias amostradas 10 vezes cada. 


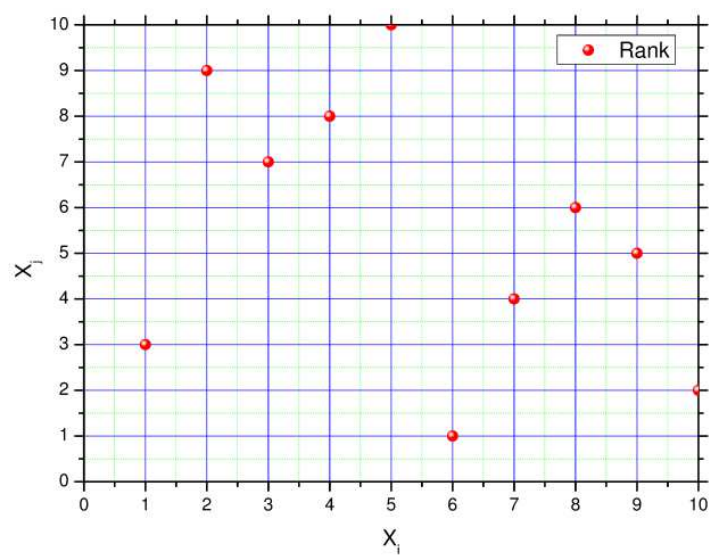

Figura 126. Dado de saída do StRAnD: Ranking dos pontos amostrais do Hipercubo Latino Gerado.

É fácil observar que cada realização das variáveis aleatórias corresponde a uma ordem, que neste caso varia de 1 a 10. 